\title{
Merging visible light with cross-coupling: the photochemical direct C-H difluoroalkylation of imidazopyrimidines
}

Chuan-Hua Qu, ${ }^{\dagger, \S}$ Gui-Ting Song, ${ }^{\dagger}{ }^{\star} \S$ Jia Xu, ${ }^{\dagger}$ Wei Yan, ${ }^{\ddagger}$ Cheng-He Zhou, ${ }^{*}$ Hong-Yu Li, ${ }^{\ddagger} * *$ Zhong-Zhu Chen, ${ }^{\dagger}, *$ and Zhi-Gang $\mathrm{Xu}^{\dagger}, *$

'International Academy of Targeted Therapeutics and Innovation, Chongqing University of Arts and Sciences. 319 Honghe Ave., Yongchuan, Chongqing, 402160, China

tDepartment of Pharmaceutical Sciences, College of Pharmacy, University of Arkansas for Medical Sciences, Little Rock, AR 72205, USA

* Institute of Bioorganic \& Medicinal Chemistry, Key Laboratory of Applied Chemistry of Chongqing Municipality, School of Chemistry and Chemical Engineering, Southwest University, Chongqing 400715, China

\section{Table of Contents}

General Information. .S2

General experimental procedure for the preparation of $\alpha$-Bromo difluoroacyl arenes............S2

Optimization of the reaction conditions of radical carbodifluoroalkylation of 2 ..................S6

General experimental procedure for the carbodifluoroalkylation of imidazopyridines...........S7

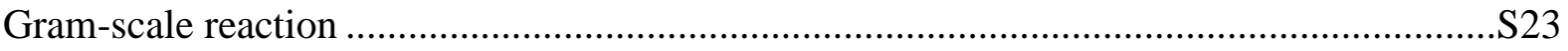

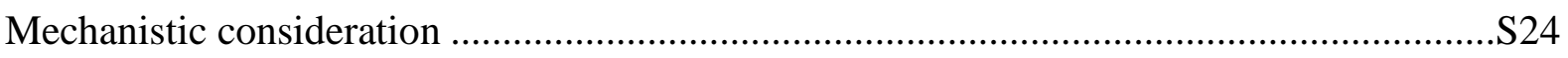

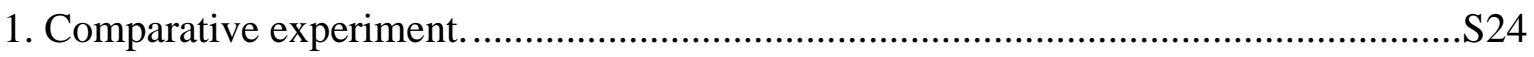

2. Radical trapping experiment with 1,1-diphenylethylene........................................S24

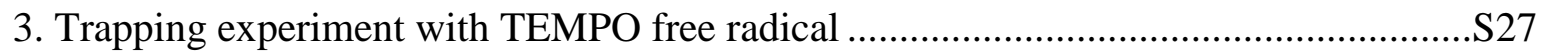

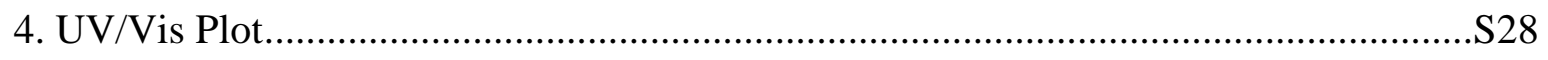

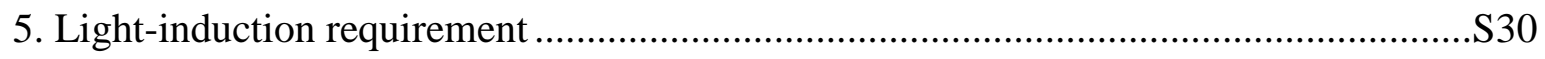

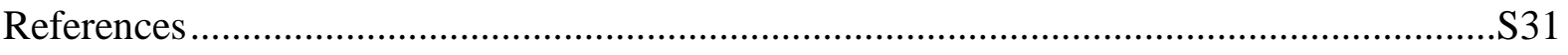

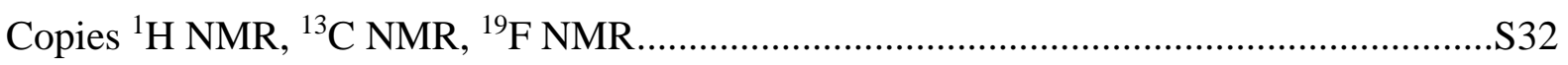




\section{General Information}

${ }^{1} \mathrm{H}$ NMR, ${ }^{19} \mathrm{~F}$ NMR and ${ }^{13} \mathrm{C}$ NMR spectra were measured on $400 \mathrm{MHz}$ spectrometer, using $\mathrm{CDCl}_{3}$ as the solvent with tetramethylsilane (TMS) as the internal standard at room temperature. Chemical shifts $(\delta)$ are given in ppm in relative to TMS, the coupling constants $J$ are given in Hz. HRMS were obtained in the ESI mode. All reactions were carried out under Ar atmosphere unless otherwise noted. All solvents were obtained from commercial suppliers. $\alpha$-Bromo difluoroacyl arenes ${ }^{1} \mathbf{2 a - 2 h}$, imidazopyridines ${ }^{2} \mathbf{1 a - 1 t}$, and $\alpha$-bromo monofluoroacetophenone ${ }^{3} \mathbf{6}$ were prepared according to the literature. Reactions were monitored by TLC on silica gelplates (GF254), and the analytical thin-layer chromatography (TLC) was performed on precoated, glass-backed silica gel plates. The 33W CFL were directly got from the supermarket.

\section{General experimental procedure for the preparation of $\alpha$-Bromo difluoroacyl arenes}

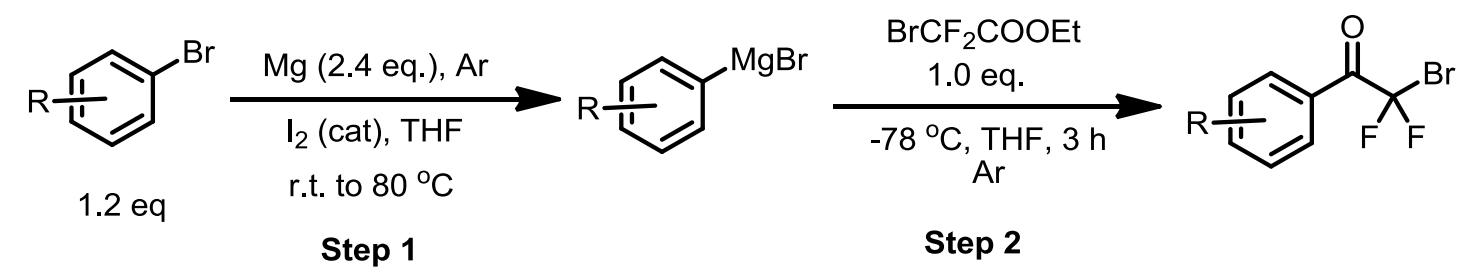

Step 1: To a flame dried $100 \mathrm{~mL}$ round bottom flask equipped with a magnetic stir bar was added 1.44 grams of magnesium $(60 \mathrm{mmol})$ and catalytic amount of iodine. To the mixture was added aryl bromide $(30 \mathrm{mmol})$ in THF $(30 \mathrm{~mL})$ dropwise via constant pressure dropping funnel under an argon atmosphere. (Note: in order to promote the initiation of Grignard reagent, we need to heat by heat dryer). Afterward, this was set to reflux for 3-5 hours.

Step 2: To a mixture of ethyl bromodifluoroacetate $(3.2 \mathrm{~mL}, 25 \mathrm{mmol})$ and THF $(30 \mathrm{~mL})$ was added a newly prepared solution of arylmagnesium bromide in THF at $-78{ }^{\circ} \mathrm{C}$ under an argon atmosphere. After the solution was stirred at that temperature for $3 \mathrm{~h}$, the mixture was quenched with $3 \mathrm{~N} \mathrm{HCl}$ and then extracted with ethyl acetate. The extract was dried over anhydrous $\mathrm{Na}_{2} \mathrm{SO}_{4}$, and the solvent was removed. Flash chromatography (silica gel, hexanes) afforded compound $\mathbf{2 a - 2 h}$. 
<smiles>O=C(c1ccccc1)C(F)(F)Br</smiles>

$2 a$<smiles>O=C(c1ccc(F)cc1)C(F)(F)Br</smiles>

$2 e$<smiles>Cc1ccc(C(=O)C(F)(F)Br)cc1</smiles>

$2 b$<smiles>O=C(c1ccc2ccccc2c1)C(F)(F)Br</smiles>

$2 \mathbf{f}$<smiles>COc1ccc(C(=O)C(F)(F)Br)cc1</smiles>

2c<smiles>CC(C)(C)c1ccc(C(=O)C(F)(F)Br)cc1</smiles>

2d

Unsuccessful substrate synthesis:<smiles>FC(F)(F)c1ccc(Br)cc1</smiles><smiles>Brc1ccc(Br)cc1</smiles><smiles>O=C(c1cccs1)C(F)(F)Br</smiles>

2g<smiles>O=C(c1ccc2c(c1)OCO2)C(F)(F)Br</smiles>

2h

The ${ }^{1} \mathrm{H}-\mathrm{NMR},{ }^{19} \mathrm{~F}$ NMR and ${ }^{13} \mathrm{C}$ NMR of 2a were reported by the reference J. Org. Chem., 2005, 70, 5912.

2-bromo-2,2-difluoro-1-(p-tolyl)ethan-1-one (2b)<smiles>Cc1ccc(C(=O)C(F)(F)Br)cc1</smiles>

2b

Colorless oil, purified by flash column chromatography over $n$-hexane, ${ }^{1} \mathrm{H}$ NMR (400 $\left.\mathrm{MHz}, \mathrm{CDCl}_{3}\right) \delta 8.03(\mathrm{~d}, J=8.1 \mathrm{~Hz}, 2 \mathrm{H}), 7.31(\mathrm{~d}, J=8.2 \mathrm{~Hz}, 2 \mathrm{H}), 2.44(\mathrm{~s}, 3 \mathrm{H}) ;{ }^{13} \mathrm{C} \mathrm{NMR}$ $\left(100 \mathrm{MHz}, \mathrm{CDCl}_{3}\right) \delta 181.0(\mathrm{t}, J=25.7 \mathrm{~Hz}), 146.6,130.8(\mathrm{t}, J=2.6 \mathrm{~Hz}), 129.7,128.8,126.6$, $113.8(\mathrm{t}, J=318.7 \mathrm{~Hz}) ;{ }^{19} \mathrm{~F} \mathrm{NMR}\left(376 \mathrm{MHz}, \mathrm{CDCl}_{3}\right) \delta-57.5$.

2-bromo-2,2-difluoro-1-(4-methoxyphenyl)ethan-1-one (2c) 
<smiles>COc1ccc(C(=O)C(F)(F)Br)cc1</smiles>

Light yellow oil, purified by flash column chromatography over $n$-hexane, ${ }^{1} \mathrm{H}$ NMR (400 $\left.\mathrm{MHz}, \mathrm{CDCl}_{3}\right) \delta 8.11(\mathrm{~d}, J=8.8 \mathrm{~Hz}, 2 \mathrm{H}), 6.98(\mathrm{~d}, J=8.9 \mathrm{~Hz}, 2 \mathrm{H}), 3.90(\mathrm{~s}, 3 \mathrm{H}) ;{ }^{13} \mathrm{C} \mathrm{NMR}$ $\left(100 \mathrm{MHz}, \mathrm{CDCl}_{3}\right) \delta 179.0(\mathrm{t}, J=25.5 \mathrm{~Hz}), 165.1,133.2$ (t, $\left.J=2.8 \mathrm{~Hz}\right), 129.4,121.7,114.3$, $113.9(\mathrm{t}, J=316.6 \mathrm{~Hz}) ;{ }^{19} \mathrm{~F} \mathrm{NMR}\left(376 \mathrm{MHz}, \mathrm{CDCl}_{3}\right) \delta-57.0$.

\section{2-bromo-1-(4-(tert-butyl)phenyl)-2,2-difluoroethan-1-one (2d)}<smiles>CC(C)(C)c1ccc(C(=O)C(F)(F)Br)cc1</smiles>

Colorless oil, purified by flash column chromatography over $n$-hexane, ${ }^{1} \mathrm{H}$ NMR (400 $\left.\mathrm{MHz}, \mathrm{CDCl}_{3}\right) \delta 8.09(\mathrm{~d}, J=8.2 \mathrm{~Hz}, 2 \mathrm{H}), 7.54(\mathrm{~d}, J=8.4 \mathrm{~Hz}, 2 \mathrm{H}), 1.35(\mathrm{~s}, 9 \mathrm{H}) ;{ }^{13} \mathrm{C} \mathrm{NMR}$ $\left(100 \mathrm{MHz}, \mathrm{CDCl}_{3}\right) \delta 181.0(\mathrm{t}, J=25.5 \mathrm{~Hz}), 159.4,131.1,130.7(\mathrm{t}, J=2.6 \mathrm{~Hz}), 126.5,126.0$, $113.8(\mathrm{t}, J=318.7 \mathrm{~Hz}), 35.4,30.92 ;{ }^{19} \mathrm{~F} \mathrm{NMR}\left(376 \mathrm{MHz}, \mathrm{CDCl}_{3}\right) \delta-57.4$.

\section{2-bromo-2,2-difluoro-1-(4-fluorophenyl)ethan-1-one (2e)}<smiles>O=C(c1ccc(F)cc1)C(F)(F)Br</smiles>

Colorless oil, purified by flash column chromatography over $n$-hexane, ${ }^{1} \mathrm{H}$ NMR (400 $\left.\mathrm{MHz}, \mathrm{CDCl}_{3}\right) \delta 8.24-8.12(\mathrm{~m}, 2 \mathrm{H}), 7.23-7.19(\mathrm{~m}, 2 \mathrm{H}) ;{ }^{13} \mathrm{C} \mathrm{NMR}\left(100 \mathrm{MHz}, \mathrm{CDCl}_{3}\right) \delta 179.9$ (t, $J=26.0 \mathrm{~Hz}), 168.1,165.5,133.6(\mathrm{dt}, J=9.9,2.9 \mathrm{~Hz}), 125.5,116.43,116.21,113.5(\mathrm{t}, J=$ $316.3 \mathrm{~Hz}) ;{ }^{19} \mathrm{~F}$ NMR $\left(376 \mathrm{MHz}, \mathrm{CDCl}_{3}\right) \delta-57.9(\mathrm{~d}, J=13.6 \mathrm{~Hz}),-97.0--107.2(\mathrm{~m})$. 


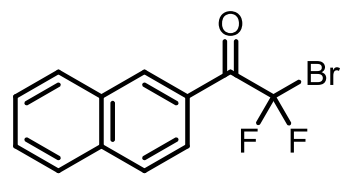

$2 f$

Colorless oil, purified by flash column chromatography over $n$-hexane, ${ }^{1} \mathrm{H}$ NMR (400 $\left.\mathrm{MHz}, \mathrm{CDCl}_{3}\right) \delta 8.7(\mathrm{~s}, 1 \mathrm{H}), 8.19-7.69(\mathrm{~m}, 4 \mathrm{H}), 7.71-7.33(\mathrm{~m}, 2 \mathrm{H}) ;{ }^{13} \mathrm{C}$ NMR $(100 \mathrm{MHz}$, $\left.\mathrm{CDCl}_{3}\right) \delta 181.4(\mathrm{t}, J=25.9 \mathrm{~Hz}), 136.3,133.5,132,130.2,129.9,128.9,127.9,127.4,126.3$, 125.9, 124.9, $113.9(\mathrm{t}, J=318.7 \mathrm{~Hz}) ;{ }^{19} \mathrm{~F} \mathrm{NMR}\left(376 \mathrm{MHz}, \mathrm{CDCl}_{3}\right) \delta-57.0$.

\section{2-bromo-2,2-difluoro-1-(thiophen-2-yl)ethan-1-one (2g)}

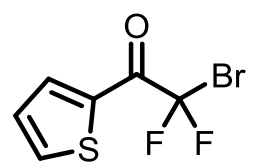

$2 \mathrm{~g}$

Light green oil, purified by flash column chromatography over $n$-hexane, ${ }^{1} \mathrm{H}$ NMR (400 $\left.\mathrm{MHz}, \mathrm{CDCl}_{3}\right) \delta 8.02(\mathrm{~s}, 1 \mathrm{H}), 7.88(\mathrm{~d}, J=4.8 \mathrm{~Hz}, 1 \mathrm{H}), 7.34-7.14(\mathrm{~m}, 1 \mathrm{H}) ;{ }^{13} \mathrm{C}$ NMR $(100$ $\left.\mathrm{MHz}, \mathrm{CDCl}_{3}\right) \delta 175.3(\mathrm{t}, J=27.9 \mathrm{~Hz}), 137.8,136.7(\mathrm{t}, J=3.7 \mathrm{~Hz}), 134.8,129.1,113.2(\mathrm{t}, J=$ $317.8 \mathrm{~Hz}) ;{ }^{19} \mathrm{~F}$ NMR $\left(376 \mathrm{MHz}, \mathrm{CDCl}_{3}\right) \delta-58.2$.

1-(benzo[d][1,3]dioxol-5-yl)-2-bromo-2,2-difluoroethan-1-one (2h)

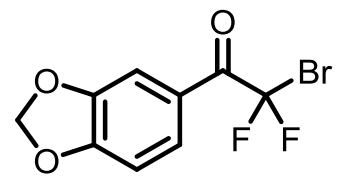

2h

Light yellow oil, purified by flash column chromatography over $n$-hexane, ${ }^{1} \mathrm{H}$ NMR (400 $\left.\mathrm{MHz}, \mathrm{CDCl}_{3}\right) \delta 7.76(\mathrm{dd}, J=28.4,9.5 \mathrm{~Hz}, 1 \mathrm{H}), 7.53(\mathrm{~s}, 1 \mathrm{H}), 6.91(\mathrm{dd}, J=15.4,10.1 \mathrm{~Hz}, 1 \mathrm{H})$, $6.10(\mathrm{~s}, 2 \mathrm{H}) ;{ }^{13} \mathrm{C} \mathrm{NMR}\left(100 \mathrm{MHz}, \mathrm{CDCl}_{3}\right) \delta 179.6(\mathrm{t}, J=25.5 \mathrm{~Hz}), 153.6,148.4,128.0(\mathrm{t}, J=$ $3.3 \mathrm{~Hz}), 123.3,113.7(\mathrm{t}, J=318.4 \mathrm{~Hz}), 109.9(\mathrm{t}, J=2.4 \mathrm{~Hz}), 108.4,102.4 ;{ }^{19} \mathrm{~F}$ NMR $(376$ $\left.\mathrm{MHz}, \mathrm{CDCl}_{3}\right) \delta-56.8$. 
Optimization of the reaction conditions of radical carbodifluoroalkylation of $2 .^{a}$<smiles>c1ccc(-c2cn3ccccc3n2)cc1</smiles>

$1 \mathrm{a}$<smiles>O=C(c1ccccc1)C(F)(F)Br</smiles>

$2 a$

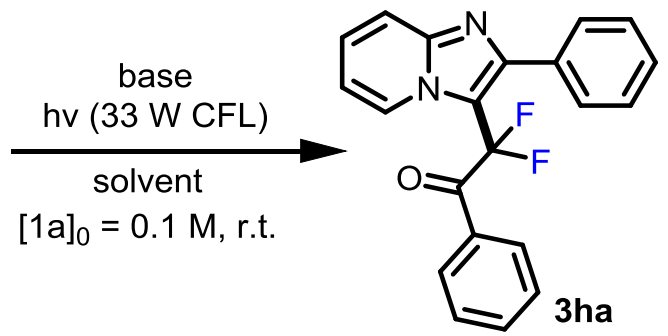

\begin{tabular}{ccccc}
\hline Entry & Base & Solvent & $\mathrm{t}[\mathrm{h}]$ & $\begin{array}{c}\text { Yield }[\%]^{b} \\
(\mathbf{3 h a})\end{array}$ \\
\hline 1 & TMEDA & MeCN & 8 & 31 \\
2 & TMEDA & MeCN & 21 & 63 \\
3 & TMEDA & MeCN & 48 & 77 \\
4 & $2,6-$ lutidine & MeCN & 48 & 40 \\
5 & Et ${ }_{3}$ N & MeCN & 48 & 63 \\
6 & DIPEA & MecN & 48 & 65 \\
7 & Cs 2 CO 3 & MeCN & 48 & 45 \\
8 & K$_{2}$ CO 3 & MeCN & 48 & $<10$ \\
9 & TMEDA & DMA & 48 & 44 \\
10 & TMEDA & DCM & 48 & 37 \\
11 & TMEDA & MTBE & 48 & 65 \\
$12^{c}$ & TMEDA & MeCN & 48 & 12 \\
$13^{d}$ & TMEDA & MTBE & 48 & 63 \\
$14^{e}$ & TMEDA & MTBE & 48 & 69 \\
$15^{f}$ & TMEDA & MTBE & 48 & 71 \\
16 & none & MTBE & 48 & $/$ \\
$17^{g}$ & TMEDA & MTBE & 48 & $/$ \\
$18^{h}$ & TMEDA & MTBE & 48 & 1 \\
\hline
\end{tabular}

${ }^{a}$ Reaction were performed on a $0.2 \mathrm{mmol}$ scale using 1.5 equiv of $\mathbf{2 a}$ and 2.0 equiv of base, $[\mathbf{1 a}]_{0}=0.1 \mathrm{M}$, and a $33 \mathrm{~W}$ CFL bulb to illuminate the reaction vessel. ${ }^{b}$ Yield are those of products isolated by column chormatography. ${ }^{c}$ Reaction in the Blue LED. ${ }^{d} 0.5$ equiv base is used. ${ }^{e} 1.0$ equiv base is used. ${ }^{f} 1.5$ equiv base is used. ${ }^{g}$ Reaction in the dark. ${ }^{h}$ Reaction performed in $80{ }^{\circ} \mathrm{C}$ without illumination of any light. DMA $=N, N$-dimethylacetamide, $\mathrm{MTBE}=$ methyl tert-butyl ether, $\mathrm{Et}_{3} \mathrm{~N}=$ triethylamine, DIPEA $=N, N$-diisopropylethylamine, TMEDA $=$ tetramethylethylenediamine. 
Other unsuccessful substrates:<smiles>O=[N+]([O-])c1ccc(-c2cn3ccccc3n2)cc1</smiles>

trace<smiles>O=[N+]([O-])c1cccn2cc(-c3ccccc3F)nc12</smiles>

mess<smiles>COc1ccc2sc3nc(-c4ccccc4)cn3c2c1</smiles>

mess

General experimental procedure for the carbodifluoroalkylation of imidazopyridines.<smiles>c1ccc(-c2cn3ccccc3n2)cc1</smiles>

1a<smiles>O=C(c1ccccc1)C(F)(F)Br</smiles>

$2 a$

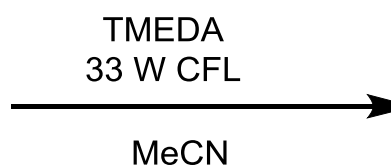

$[1 \mathrm{a}]_{0}=0.1 \mathrm{M}$, r.t.

$48 \mathrm{~h}$

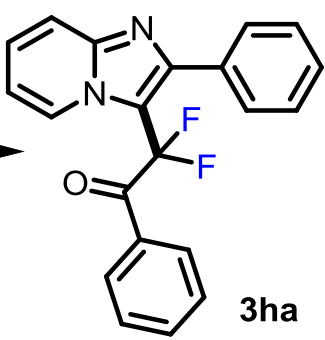

Tetramethylethylenediamine $(61 \mu \mathrm{L}, 0.4 \mathrm{mmol}, 2.0$ eq), imidazopyridines $1 \mathrm{a}$ (39 mg, 0.2 mmol, 1.0 eq) and $\alpha$-bromo difluoroacylarenes $2 \mathbf{a}(71 \mathrm{mg}, 0.3 \mathrm{mmol}, 1.5 \mathrm{eq})$ were added to a flame-dried Schlenk flask containing a stirring bar and purged by evacuating the flask and backfilling with argon three times. In the absence of light, anhydrous acetonitrile $(2 \mathrm{ml}, 0.1$ M) was added and the flask was sealed. The mixture was then stirred under irradiation from $33 \mathrm{~W}$ CFL. After $48 \mathrm{~h}$, the crude products were purified by column chromatography over silica gel using hexanes/EtOAc as eluent to yield 3ha (54 mg, 77\%).

\section{2,2-difluoro-2-(8-methyl-2-phenylimidazo[1,2-a]pyridin-3-yl)-1-phenylethan-1-} one (3aa)

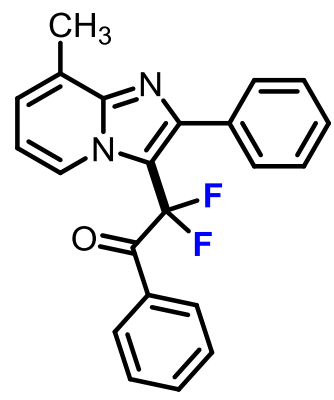


$43 \mathrm{~h}, 60 \mathrm{mg}, 80 \%$ yield, colorless oil, $\mathrm{R}_{\mathrm{f}}=0.4$ (ethyl acetate /hexane $=20 \%$ ) ${ }^{1} \mathrm{H}$ NMR $\left(400 \mathrm{MHz}, \mathrm{CDCl}_{3}\right): \delta 8.38(\mathrm{~d}, J=6.8 \mathrm{~Hz}, 1 \mathrm{H}), 7.64(\mathrm{~d}, J=8.0 \mathrm{~Hz}, 2 \mathrm{H}), 7.45(\mathrm{t}, J=7.2 \mathrm{~Hz}$, 1H), $7.37(\mathrm{~d}, J=6.8 \mathrm{~Hz}, 2 \mathrm{H}), 7.34-7.25(\mathrm{~m}, 3 \mathrm{H}), 7.21(\mathrm{t}, J=8.0 \mathrm{~Hz}, 2 \mathrm{H}), 7.15(\mathrm{~d}, J=6.8$ $\mathrm{Hz}, 1 \mathrm{H}), 6.87(\mathrm{t}, J=6.8 \mathrm{~Hz}, 1 \mathrm{H}), 2.64(\mathrm{~s}, 3 \mathrm{H}) ;{ }^{13} \mathrm{C} \mathrm{NMR}\left(100 \mathrm{MHz}, \mathrm{CDCl}_{3}\right): \delta 186.0(\mathrm{t}, J=$ $29.9 \mathrm{~Hz}), 147.8$ (t, $J=3.3 \mathrm{~Hz}), 146.8,134.2,133.4,131.5,130.4,130.0-129.6$ (m), 128.7, 128.3, 127.9, 127.9, 125.6, $124.2(\mathrm{t}, J=5.0 \mathrm{~Hz}), 113.7,114.5(\mathrm{t}, J=247.5 \mathrm{~Hz}), 112.6(\mathrm{t}, J=$ $30.8 \mathrm{~Hz}), 17.2 ;{ }^{19} \mathrm{~F}$ NMR (376 MHz, $\left.\mathrm{CDCl}_{3}\right): \delta-94.2$; HRMS (ESI): exact mass calculated for $[\mathrm{M}+\mathrm{H}]^{+}\left(\mathrm{C}_{22} \mathrm{H}_{17} \mathrm{~F}_{2} \mathrm{~N}_{2} \mathrm{O}^{+}\right)$requires $m / z$ 363.1303, found $m / z$ 363.1302.

\section{2,2-difluoro-2-(2-(4-methoxyphenyl)-8-methylimidazo[1,2-a]pyridin-3-yl)-1-phenylethan} -1-one (3ab)

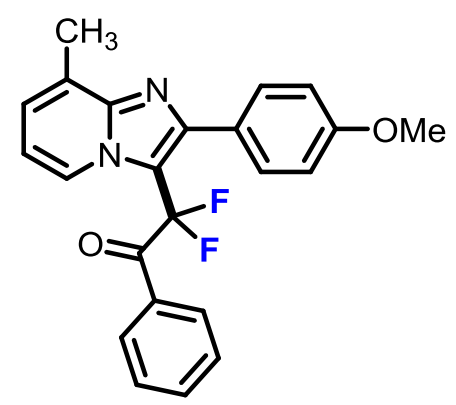

$43 \mathrm{~h}, 57 \mathrm{mg}, 73 \%$ yield, colorless oil, $\mathrm{R}_{\mathrm{f}}=0.3$ (ethyl acetate $/$ hexane $=25 \%$ ); ${ }^{1} \mathrm{H}$ NMR $\left(400 \mathrm{MHz}, \mathrm{CDCl}_{3}\right): \delta 8.38(\mathrm{~d}, J=6.8 \mathrm{~Hz}, 1 \mathrm{H}), 7.65(\mathrm{~d}, J=8.0 \mathrm{~Hz}, 2 \mathrm{H}), 7.46(\mathrm{t}, J=6.8 \mathrm{~Hz}$, 1H), 7.31-7.25 (m, 2H), $7.22(\mathrm{t}, J=8.0 \mathrm{~Hz}, 2 \mathrm{H}), 7.15(\mathrm{~d}, J=6.8 \mathrm{~Hz}, 1 \mathrm{H}), 6.87(\mathrm{t}, J=6.8 \mathrm{~Hz}$, $1 \mathrm{H}), 6.81(\mathrm{~d}, J=8.8 \mathrm{~Hz}, 2 \mathrm{H}), 3.82(\mathrm{~s}, 3 \mathrm{H}), 2.64(\mathrm{~s}, 3 \mathrm{H}) ;{ }^{13} \mathrm{C} \mathrm{NMR}\left(100 \mathrm{MHz}, \mathrm{CDCl}_{3}\right): \delta$ $186.0(\mathrm{t}, J=30.7 \mathrm{~Hz}), 160.1,147.7,146.8,134.2,131.7,129.8,128.3,127.8,125.7,125.5$, 124.2, $114.4(\mathrm{t}, J=273.9 \mathrm{~Hz}), 113.4,55.3,17.22 ;{ }^{19} \mathrm{~F}$ NMR (376 MHz, $\left.\mathrm{CDCl}_{3}\right): \delta-94.0$; HRMS (ESI): exact mass calculated for $[\mathrm{M}+\mathrm{H}]^{+}\left(\mathrm{C}_{23} \mathrm{H}_{19} \mathrm{~F}_{2} \mathrm{~N}_{2} \mathrm{O}_{2}{ }^{+}\right)$requires $\mathrm{m} / \mathrm{z}$ 393.1409, found $m / z$ 393.1462.

\section{2,2-difluoro-2-(2-(2-fluorophenyl)-8-methylimidazo[1,2-a]pyridin-3-yl)-1-phenylethan-1} -one (3ac) 


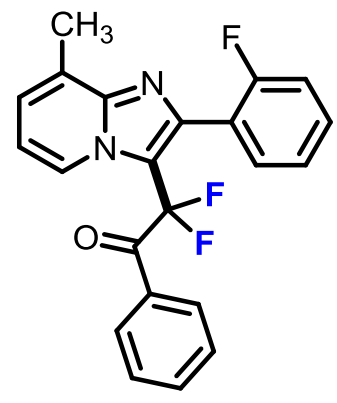

48 h, $62 \mathrm{mg}, 82 \%$ yield, colorless oil, $\mathrm{R}_{\mathrm{f}}=0.4$ (ethyl acetate $/$ hexane $=20 \%$ ); ${ }^{1} \mathrm{H}$ NMR $\left(400 \mathrm{MHz}, \mathrm{CDCl}_{3}\right): \delta 8.30(\mathrm{~d}, J=6.8 \mathrm{~Hz}, 1 \mathrm{H}), 7.83(\mathrm{~d}, J=8.0 \mathrm{~Hz}, 2 \mathrm{H}), 7.54(\mathrm{t}, J=7.2 \mathrm{~Hz}$, 1H), 7.42-7.27 (m, 4H), $7.16(\mathrm{~d}, J=6.8 \mathrm{~Hz}, 1 \mathrm{H}), 7.11(\mathrm{t}, J=7.2 \mathrm{~Hz}, 1 \mathrm{H}), 6.95(\mathrm{t}, J=9.2 \mathrm{~Hz}$, $1 \mathrm{H}), 6.87(\mathrm{t}, J=6.8 \mathrm{~Hz}, 1 \mathrm{H}), 2.65(\mathrm{~s}, 3 \mathrm{H}) ;{ }^{13} \mathrm{C} \mathrm{NMR}\left(100 \mathrm{MHz}, \mathrm{CDCl}_{3}\right): \delta 186.3(\mathrm{t}, J=31.0$ Hz), 161.5, 159.0, 147.0, 140.9, 134.4, 132.4, 131.6, 130.7 (d, $J=8.1 \mathrm{~Hz}), 129.9$ (t, $J=2.8$ $\mathrm{Hz}), 128.5,128.0,125.6,124.0(\mathrm{t}, J=4.4 \mathrm{~Hz}), 123.8(\mathrm{~d}, J=3.7 \mathrm{~Hz}), 121.7(\mathrm{~d}, J=15.0 \mathrm{~Hz})$, $115.4(\mathrm{~d}, J=21.6 \mathrm{~Hz}), 114.7(\mathrm{t}, J=248.9 \mathrm{~Hz}), 113.74,17.23 ;{ }^{19} \mathrm{~F} \mathrm{NMR}\left(376 \mathrm{MHz}, \mathrm{CDCl}_{3}\right)$ : $\delta-96.3(\mathrm{~d}, J=4.9 \mathrm{~Hz}),-113.3--113.4(\mathrm{~m})$; HRMS (ESI): exact mass calculated for $[\mathrm{M}+\mathrm{H}]^{+}$ $\left(\mathrm{C}_{22} \mathrm{H}_{16} \mathrm{~F}_{3} \mathrm{~N}_{2} \mathrm{O}^{+}\right)$requires $\mathrm{m} / \mathrm{z}$ 381.1209, found $\mathrm{m} / \mathrm{z} 381.1209$.

\section{2-(8-bromo-2-phenylimidazo[1,2-a]pyridin-3-yl)-2,2-difluoro-1-phenylethan-1-} one (3ba)

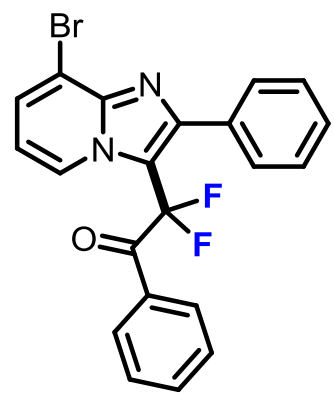

$56 \mathrm{~h}, 60 \mathrm{mg}, 71 \%$ yield, colorless oil, $\mathrm{R}_{\mathrm{f}}=0.4$ (ethyl acetate $/$ hexane $=20 \%$ ); ${ }^{1} \mathrm{H}$ NMR $\left(400 \mathrm{MHz}, \mathrm{CDCl}_{3}\right): \delta 8.50(\mathrm{~d}, J=6.8 \mathrm{~Hz}, 1 \mathrm{H}), 7.65(\mathrm{~d}, J=7.6 \mathrm{~Hz}, 3 \mathrm{H}), 7.49(\mathrm{t}, J=7.6 \mathrm{~Hz}$, 1H), $7.40(\mathrm{~d}, J=7.2 \mathrm{~Hz}, 2 \mathrm{H}), 7.35-7.22(\mathrm{~m}, 5 \mathrm{H}), 6.86(\mathrm{t}, J=7.2 \mathrm{~Hz}, 1 \mathrm{H}) ;{ }^{13} \mathrm{C} \mathrm{NMR}(100$ $\left.\mathrm{MHz}, \mathrm{CDCl}_{3}\right): \delta 185.8(\mathrm{t}, J=29.8 \mathrm{~Hz}), 148.8,144.4,134.5,132.7,131.2,130.4,129.9,129.3$, 129.0, 128.5, 127.9, $125.8(\mathrm{t}, J=5.0 \mathrm{~Hz}), 114.2(\mathrm{t}, J=248.6 \mathrm{~Hz}), 113.7,111.9 ;{ }^{19} \mathrm{~F}$ NMR 
$\left(376 \mathrm{MHz}, \mathrm{CDCl}_{3}\right): \delta$-94.4 ; HRMS (ESI): exact mass calculated for $[\mathrm{M}+\mathrm{H}]^{+}$ $\left(\mathrm{C}_{21} \mathrm{H}_{14} \mathrm{BrF}_{2} \mathrm{~N}_{2} \mathrm{O}^{+}\right)$requires $m / z$ 427.0252, found $m / z 427.0252$.

\section{2-(8-bromo-2-(4-methoxyphenyl)imidazo[1,2-a]pyridin-3-yl)-2,2-difluoro-1-phenylethan}

-1-one (3bb)

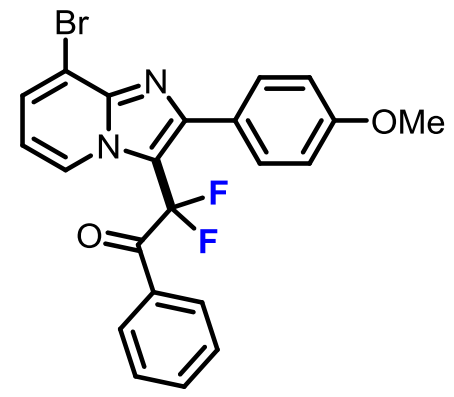

69 h, $80 \mathrm{mg}, 88 \%$ yield, colorless oil, $\mathrm{R}_{\mathrm{f}}=0.3$ (ethyl acetate $/$ hexane $=25 \%$ ); ${ }^{1} \mathrm{H}$ NMR $\left(400 \mathrm{MHz}, \mathrm{CDCl}_{3}\right): \delta 8.49(\mathrm{~d}, J=6.8 \mathrm{~Hz}, 1 \mathrm{H}), 7.64(\mathrm{t}, J=7.6 \mathrm{~Hz}, 3 \mathrm{H}), 7.48(\mathrm{t}, J=7.2 \mathrm{~Hz}$, 1H), $7.32(\mathrm{~d}, J=8.4 \mathrm{~Hz}, 2 \mathrm{H}), 7.26-7.22(\mathrm{~m}, 2 \mathrm{H}), 6.83(\mathrm{dd}, J=14.4,7.2 \mathrm{~Hz}, 3 \mathrm{H}), 3.82$ (s, $3 \mathrm{H}) ;{ }^{13} \mathrm{C} \mathrm{NMR}\left(100 \mathrm{MHz}, \mathrm{CDCl}_{3}\right): \delta 185.7(\mathrm{t}, J=29.9 \mathrm{~Hz}), 160.3,148.7,144.3,134.4$, 131.8, 131.3, $129.7(\mathrm{t}, J=2.5 \mathrm{~Hz}), 129.3,128.4,125.8(\mathrm{t}, J=5.0 \mathrm{~Hz}), 125.0,114.2(\mathrm{t}, J=$ $248.3 \mathrm{~Hz}), 113.8,113.6,113.4,111.8,55.3 ;{ }^{19} \mathrm{~F}$ NMR (376 MHz, $\left.\mathrm{CDCl}_{3}\right): \delta$-94.2 ; HRMS (ESI): exact mass calculated for $[\mathrm{M}+\mathrm{H}]^{+}\left(\mathrm{C}_{22} \mathrm{H}_{16} \mathrm{BrF}_{2} \mathrm{~N}_{2} \mathrm{O}_{2}{ }^{+}\right)$requires $\mathrm{m} / \mathrm{z}$ 457.0358, found $m / z 457.0349$.

\section{4-(8-bromo-3-(1,1-difluoro-2-oxo-2-phenylethyl)imidazo[1,2-a]pyridin-2-yl)benzonitrile} (3bc)

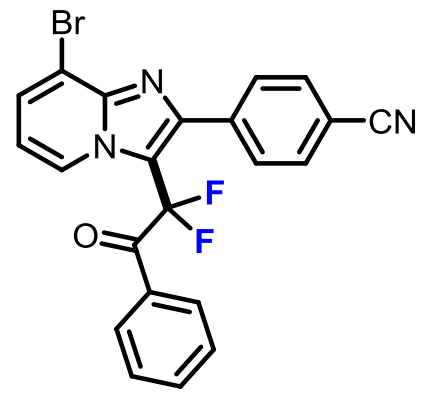

69 h, $69 \mathrm{mg}, 77 \%$ yield, colorless oil, $\mathrm{R}_{\mathrm{f}}=0.5$ (ethyl acetate $/$ hexane $=20 \%$ ); ${ }^{1} \mathrm{H}$ NMR (400 MHz, $\left.\mathrm{CDCl}_{3}\right): \delta 8.43(\mathrm{~d}, J=6.8 \mathrm{~Hz}, 1 \mathrm{H}), 7.74(\mathrm{~d}, J=8.0 \mathrm{~Hz}, 2 \mathrm{H}), 7.69(\mathrm{~d}, J=7.2 \mathrm{~Hz}$, 1H), $7.61(\mathrm{~d}, J=8.4 \mathrm{~Hz}, 2 \mathrm{H}), 7.59-7.55(\mathrm{~m}, 3 \mathrm{H}), 7.33(\mathrm{t}, J=7.6 \mathrm{~Hz}, 2 \mathrm{H}), 6.90(\mathrm{t}, J=7.2 \mathrm{~Hz}$, 
$1 \mathrm{H}) ;{ }^{13} \mathrm{C} \mathrm{NMR}\left(100 \mathrm{MHz}, \mathrm{CDCl}_{3}\right): \delta 185.8(\mathrm{t}, J=30.4 \mathrm{~Hz}), 146.4,144.6,137.5,135.0$, 131.7, 131.0, 130.0, 129.8, 128.7, 125.7 (t, $J=4.6 \mathrm{~Hz}), 118.6,114.7,114.3$ (t, $J=250.5 \mathrm{~Hz})$, 114.2, 112.6, $112.1 ;{ }^{19} \mathrm{~F}$ NMR (376 $\mathrm{MHz}, \mathrm{CDCl}_{3}$ ): $\delta$-94.5 ; HRMS (ESI): exact mass calculated for $[\mathrm{M}+\mathrm{H}]^{+}\left(\mathrm{C}_{23} \mathrm{H}_{18} \mathrm{BrF}_{2} \mathrm{~N}_{2} \mathrm{O}_{2}{ }^{+}\right)$requires $\mathrm{m} / z$ 452.0205, found $m / z$ 452.0201.

2-(8-bromo-2-(2-fluorophenyl)imidazo[1,2-a]pyridin-3-yl)-2,2-difluoro-1-phenylethan-1one (3bd)

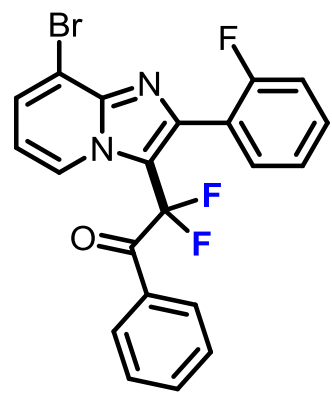

$72 \mathrm{~h}, 59 \mathrm{mg}, 67 \%$ yield, colorless oil, $\mathrm{R}_{\mathrm{f}}=0.5$ (ethyl acetate $/$ hexane $=20 \%$ ); ${ }^{1} \mathrm{H}$ NMR $\left(400 \mathrm{MHz}, \mathrm{CDCl}_{3}\right): \delta 8.39(\mathrm{~d}, J=6.8 \mathrm{~Hz}, 1 \mathrm{H}), 7.87(\mathrm{~d}, J=7.6 \mathrm{~Hz}, 2 \mathrm{H}), 7.64(\mathrm{~d}, J=7.2 \mathrm{~Hz}$, 1H), $7.58(\mathrm{t}, J=7.6 \mathrm{~Hz}, 1 \mathrm{H}), 7.46(\mathrm{td}, J=7.6,1.6 \mathrm{~Hz}, 1 \mathrm{H}), 7.41-7.29(\mathrm{~m}, 3 \mathrm{H}), 7.13(\mathrm{t}, J=$ $7.6 \mathrm{~Hz}, 1 \mathrm{H}), 6.96(\mathrm{t}, J=8.8 \mathrm{~Hz}, 1 \mathrm{H}), 6.85(\mathrm{t}, J=7.2 \mathrm{~Hz}, 1 \mathrm{H}) ;{ }^{13} \mathrm{C} \mathrm{NMR}\left(100 \mathrm{MHz}, \mathrm{CDCl}_{3}\right)$ : $\delta 186.4(\mathrm{t}, J=31.0 \mathrm{~Hz}), 161.4,159.0,144.6,141.8,134.7,132.3,131.3,131.0(\mathrm{~d}, J=8.4 \mathrm{~Hz})$, 130.0, 129.2, 128.6, $125.6(\mathrm{t}, J=4.3 \mathrm{~Hz}), 123.9(\mathrm{~d}, J=3.7 \mathrm{~Hz}), 121.2(\mathrm{~d}, J=14.7 \mathrm{~Hz}), 115.3$ $(\mathrm{d}, J=21.6 \mathrm{~Hz}), 114.6(\mathrm{t}, J=250.6 \mathrm{~Hz}), 113.7,111.95 ;{ }^{19} \mathrm{~F}$ NMR $\left(376 \mathrm{MHz}, \mathrm{CDCl}_{3}\right): \delta-96.8$ $(\mathrm{d}, J=5.6 \mathrm{~Hz}),-112.7--114.9(\mathrm{~m})$; HRMS (ESI): exact mass calculated for $[\mathrm{M}+\mathrm{H}]^{+}$ $\left(\mathrm{C}_{21} \mathrm{H}_{13} \mathrm{BrF}_{3} \mathrm{~N}_{2} \mathrm{O}^{+}\right)$requires $\mathrm{m} / z$ 445.0158, found $\mathrm{m} / \mathrm{z} 445.0159$.

ethyl 8-bromo-3-(1,1-difluoro-2-oxo-2-phenylethyl)imidazo[1,2-a $]$ pyridine-2carboxylate (3c) 


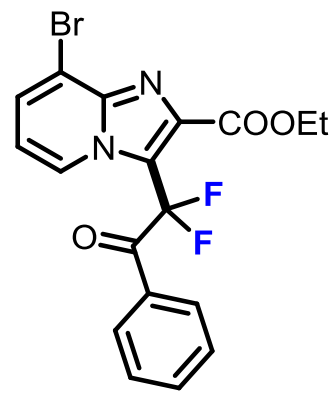

$72 \mathrm{~h}, 39 \mathrm{mg}, 46 \%$ yield, light yellow oil oil, $\mathrm{R}_{\mathrm{f}}=0.5$ (ethyl acetate $/$ hexane $=20 \%$ ); ${ }^{1} \mathrm{H}$ $\operatorname{NMR}\left(400 \mathrm{MHz}, \mathrm{CDCl}_{3}\right): \delta 8.60(\mathrm{~d}, J=7.2 \mathrm{~Hz}, 1 \mathrm{H}), 8.08(\mathrm{~d}, J=7.6 \mathrm{~Hz}, 2 \mathrm{H}), 7.69(\mathrm{~d}, J=$ $7.2 \mathrm{~Hz}, 1 \mathrm{H}), 7.64(\mathrm{t}, J=7.6 \mathrm{~Hz}, 1 \mathrm{H}), 7.48(\mathrm{t}, J=8.0 \mathrm{~Hz}, 2 \mathrm{H}), 6.92(\mathrm{t}, J=7.2 \mathrm{~Hz}, 1 \mathrm{H}), 4.26(\mathrm{q}$, $J=7.2 \mathrm{~Hz}, 2 \mathrm{H}), 1.23(\mathrm{t}, J=7.2 \mathrm{~Hz}, 3 \mathrm{H}) ;{ }^{13} \mathrm{C} \mathrm{NMR}\left(100 \mathrm{MHz}, \mathrm{CDCl}_{3}\right): \delta 185.6(\mathrm{t}, J=30.1$ $\mathrm{Hz}), 162.2,143.9,137.7,134.4,132.1,129.9,128.7,126.1(\mathrm{t}, J=8.6 \mathrm{~Hz}), 115.1,114.5$ (t, $J=$ 251.8 Hz), 113.3, 62.1, 14.0; ${ }^{19} \mathrm{~F}$ NMR (376 MHz, $\left.\mathrm{CDCl}_{3}\right): \delta$-93.4; HRMS (ESI): exact mass calculated for $[\mathrm{M}+\mathrm{H}]^{+}\left(\mathrm{C}_{18} \mathrm{H}_{14} \mathrm{BrF}_{2} \mathrm{~N}_{2} \mathrm{O}_{3}{ }^{+}\right)$requires $m / z$ 423.0150, found $m / z 423.0167$.

\section{2-(7-chloro-2-(2-fluorophenyl)imidazo[1,2-a]pyridin-3-yl)-2,2-difluoro-1-phenylethan-1-} one (3d)

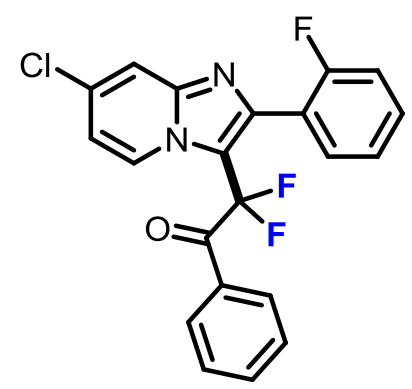

$69 \mathrm{~h}, 58 \mathrm{mg}, 72 \%$ yield, colorless oil, $\mathrm{R}_{\mathrm{f}}=0.5$ (ethyl acetate $/$ hexane $=20 \%$ ); ${ }^{1} \mathrm{H}$ NMR $\left(400 \mathrm{MHz}, \mathrm{CDCl}_{3}\right): \delta 8.32(\mathrm{~d}, J=7.6 \mathrm{~Hz}, 1 \mathrm{H}), 7.87(\mathrm{~d}, J=8.0 \mathrm{~Hz}, 2 \mathrm{H}), 7.71(\mathrm{~d}, J=1.6 \mathrm{~Hz}$, 1H), $7.58(\mathrm{t}, J=7.6 \mathrm{~Hz}, 1 \mathrm{H}), 7.46-7.30(\mathrm{~m}, 4 \mathrm{H}), 7.15(\mathrm{t}, J=7.6 \mathrm{~Hz}, 1 \mathrm{H}), 7.01-6.92(\mathrm{~m}, 2 \mathrm{H})$; ${ }^{13} \mathrm{C}$ NMR $\left(100 \mathrm{MHz}, \mathrm{CDCl}_{3}\right): \delta 186.6(\mathrm{t}, J=31.5 \mathrm{~Hz}), 161.3,158.8,146.4,142.2,134.7$, 133.6, $132.0(\mathrm{~d}, J=2.0 \mathrm{~Hz}), 131.4(\mathrm{t}, J=1.7 \mathrm{~Hz}), 131.0(\mathrm{~d}, J=8.1 \mathrm{~Hz}), 129.9(\mathrm{t}, J=2.8 \mathrm{~Hz})$, 128.6, $126.6(\mathrm{t}, J=4.4 \mathrm{~Hz}), 123.9(\mathrm{~d}, J=3.7 \mathrm{~Hz}), 121.1(\mathrm{~d}, J=14.7 \mathrm{~Hz}), 116.8,115.5,115.3$, $114.7(\mathrm{t}, J=250.4 \mathrm{~Hz}), 114.4,112.2 ;{ }^{19} \mathrm{~F} \mathrm{NMR}\left(376 \mathrm{MHz}, \mathrm{CDCl}_{3}\right): \delta-96.4(\mathrm{~d}, J=6.0 \mathrm{~Hz})$, 
-111.5--117.1 (m); HRMS (ESI): exact mass calculated for $[\mathrm{M}+\mathrm{H}]^{+}\left(\mathrm{C}_{21} \mathrm{H}_{13} \mathrm{ClF}_{3} \mathrm{~N}_{2} \mathrm{O}^{+}\right)$ requires $m / z$ 401.0663, found $m / z 401.0669$.

2-(8-bromo-6-methyl-2-phenylimidazo[1,2-a]pyridin-3-yl)-2,2-difluoro-1-phenylethan-1one (3ea)

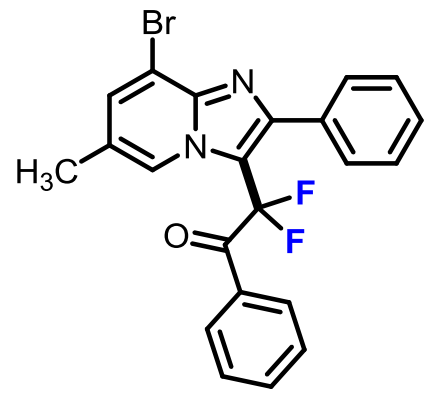

$56 \mathrm{~h}, 60 \mathrm{mg}, 68 \%$ yield, colorless oil, $\mathrm{R}_{\mathrm{f}}=0.5$ (ethyl acetate $/$ hexane $=20 \%$ ); ${ }^{1} \mathrm{H}$ NMR (400 MHz, $\left.\mathrm{CDCl}_{3}\right): \delta 8.27(\mathrm{~s}, 1 \mathrm{H}), 7.64(\mathrm{~d}, J=7.6 \mathrm{~Hz}, 2 \mathrm{H}), 7.52(\mathrm{~s}, 1 \mathrm{H}), 7.48(\mathrm{t}, J=7.2 \mathrm{~Hz}$, $1 \mathrm{H}), 7.37(\mathrm{~d}, J=6.8 \mathrm{~Hz}, 2 \mathrm{H}), 7.32(\mathrm{~d}, J=6.8 \mathrm{~Hz}, 1 \mathrm{H}), 7.30-7.20(\mathrm{~m}, 4 \mathrm{H}), 2.41(\mathrm{~s}, 3 \mathrm{H}) ;{ }^{13} \mathrm{C}$ NMR (100 MHz, $\left.\mathrm{CDCl}_{3}\right): \delta 185.8(\mathrm{t}, J=29.9 \mathrm{~Hz}), 148.6,143.5,134.4,132.8,132.3,131.3$, $130.4,129.8,128.8,128.4,127.9,123.8,123.5(\mathrm{t}, J=5.0 \mathrm{~Hz}), 114.2(\mathrm{t}, J=248.6 \mathrm{~Hz}), 111.2$, $18.3 ;{ }^{19} \mathrm{~F} \mathrm{NMR}\left(376 \mathrm{MHz}, \mathrm{CDCl}_{3}\right): \delta-94.3$; HRMS (ESI): exact mass calculated for $[\mathrm{M}+\mathrm{H}]^{+}$ $\left(\mathrm{C}_{22} \mathrm{H}_{16} \mathrm{BrF}_{2} \mathrm{~N}_{2} \mathrm{O}^{+}\right)$requires $m / z$ 441.0409, found $\mathrm{m} / \mathrm{z} 441.0410$.

2-(8-bromo-2-(4-methoxyphenyl)-6-methylimidazo[1,2-a]pyridin-3-yl)-2,2-difluoro-1-ph enylethan-1-one (3eb)

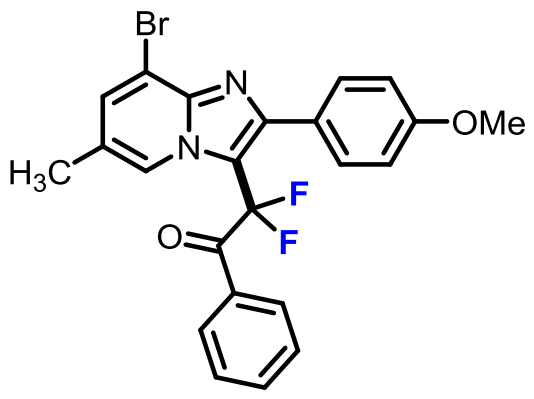

$69 \mathrm{~h}, 81 \mathrm{mg}, 86 \%$ yield, colorless oil, $\mathrm{R}_{\mathrm{f}}=0.3$ (ethyl acetate $/$ hexane $=25 \%$ ); ${ }^{1} \mathrm{H}$ NMR (400 MHz, $\left.\mathrm{CDCl}_{3}\right): \delta 8.27(\mathrm{~s}, 1 \mathrm{H}), 7.63(\mathrm{~d}, J=7.6 \mathrm{~Hz}, 2 \mathrm{H}), 7.50(\mathrm{~s}, 1 \mathrm{H}), 7.47$ (t, $J=7.6 \mathrm{~Hz}$, 1H), $7.29(\mathrm{~d}, J=8.4 \mathrm{~Hz}, 2 \mathrm{H}), 7.23(\mathrm{t}, J=7.6 \mathrm{~Hz}, 2 \mathrm{H}), 6.79(\mathrm{~d}, J=8.8 \mathrm{~Hz}, 2 \mathrm{H}), 3.81(\mathrm{~s}, 3 \mathrm{H})$, 
$2.40(\mathrm{~s}, 3 \mathrm{H}) ;{ }^{13} \mathrm{C} \mathrm{NMR}\left(100 \mathrm{MHz}, \mathrm{CDCl}_{3}\right): \delta 185.7(\mathrm{t}, J=29.5 \mathrm{~Hz}), 160.2,148.4,143.4$, 134.4, 132.2, 131.7, 131.4, $129.7(\mathrm{t}, J=2.2 \mathrm{~Hz}), 128.4,125.1,123.7,123.5(\mathrm{t}, J=5.0 \mathrm{~Hz})$, $114.2(\mathrm{t}, J=247.9 \mathrm{~Hz}), 113.5(\mathrm{t}, J=30.5 \mathrm{~Hz}), 113.3(\mathrm{~d}, J=15.4 \mathrm{~Hz}), 111.0,55.3,18.2 ;{ }^{19} \mathrm{~F}$ NMR (376 MHz, $\left.\mathrm{CDCl}_{3}\right): \delta$-94.1 ; HRMS (ESI): exact mass calculated for $[\mathrm{M}+\mathrm{H}]^{+}$ $\left(\mathrm{C}_{23} \mathrm{H}_{18} \mathrm{BrF}_{2} \mathrm{~N}_{2} \mathrm{O}_{2}{ }^{+}\right)$requires $m / z$ 471.0514, found $m / z 471.0498$.

\section{2,2-difluoro-1-phenyl-2-(6-phenylimidazo[2,1-b]thiazol-5-yl)ethan-1-one (3fa)}

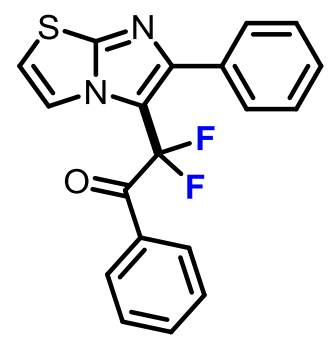

$48 \mathrm{~h}, 55 \mathrm{mg}, 78 \%$ yield, light yellow oil, $\mathrm{R}_{\mathrm{f}}=0.5$ (ethyl acetate $/$ hexane $=20 \%$ ); ${ }^{1} \mathrm{H}$ NMR (400 MHz, $\left.\mathrm{CDCl}_{3}\right): \delta 7.73(\mathrm{~d}, J=4.0 \mathrm{~Hz}, 1 \mathrm{H}), 7.64(\mathrm{~d}, J=7.6 \mathrm{~Hz}, 2 \mathrm{H}), 7.51-7.45(\mathrm{~m}, 1 \mathrm{H})$, $7.40(\mathrm{~d}, J=5.6 \mathrm{~Hz}, 2 \mathrm{H}), 7.35-7.21(\mathrm{~m}, 5 \mathrm{H}), 6.96(\mathrm{~d}, J=3.6 \mathrm{~Hz}, 1 \mathrm{H}) ;{ }^{13} \mathrm{C} \mathrm{NMR}(100 \mathrm{MHz}$, $\left.\mathrm{CDCl}_{3}\right): \delta 186.4(\mathrm{t}, J=29.7 \mathrm{~Hz}), 151.9,149.3,134.3(\mathrm{~d}, J=8.1 \mathrm{~Hz}), 132.9,131.4,129.7$, $129.6,128.7,128.6,128.4,128.4,128.1,128.0,119.7,114.4(\mathrm{t}, J=31.7 \mathrm{~Hz}), 113.9(\mathrm{t}, J=$ $248.6 \mathrm{~Hz}), 113.81 ;{ }^{19} \mathrm{~F}$ NMR (376 MHz, $\left.\mathrm{CDCl}_{3}\right): \delta$-92.6 ; HRMS (ESI): exact mass calculated for $[\mathrm{M}+\mathrm{H}]^{+}\left(\mathrm{C}_{19} \mathrm{H}_{13} \mathrm{~F}_{2} \mathrm{~N}_{2} \mathrm{OS}^{+}\right)$requires $m / z$ 355.0711, found $m / z$ 355.0710.

\section{2,2-difluoro-2-(6-(4-methoxyphenyl)imidazo[2,1-b]thiazol-5-yl)-1-phenylethan-1-one} (3fb)

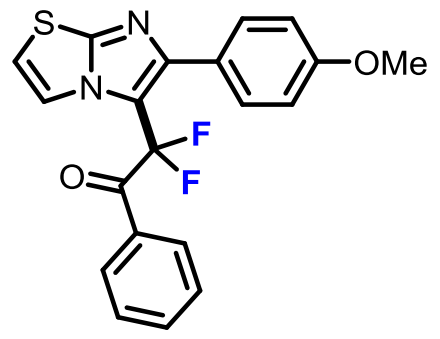

$48 \mathrm{~h}, 57 \mathrm{mg}, 75 \%$ yield, light brown oil, $\mathrm{R}_{\mathrm{f}}=0.4$ (ethyl acetate $/$ hexane $=25 \%$ ); ${ }^{1} \mathrm{H}$ NMR $\left(400 \mathrm{MHz}, \mathrm{CDCl}_{3}\right): \delta 7.72(\mathrm{~d}, J=4.0 \mathrm{~Hz}, 1 \mathrm{H}), 7.64(\mathrm{~d}, J=7.6 \mathrm{~Hz}, 2 \mathrm{H}), 7.48(\mathrm{t}, J=7.2 \mathrm{~Hz}$, $1 \mathrm{H}), 7.33(\mathrm{~d}, J=8.4 \mathrm{~Hz}, 2 \mathrm{H}), 7.25(\mathrm{t}, J=6.4 \mathrm{~Hz}, 2 \mathrm{H}), 6.95(\mathrm{dd}, J=4.4,0.8 \mathrm{~Hz}, 1 \mathrm{H}), 6.83(\mathrm{~d}$, 
$J=7.6 \mathrm{~Hz}, 2 \mathrm{H}), 3.82(\mathrm{~s}, 3 \mathrm{H}) ;{ }^{13} \mathrm{C} \mathrm{NMR}\left(100 \mathrm{MHz}, \mathrm{CDCl}_{3}\right): \delta 186.4(\mathrm{t}, J=30.4 \mathrm{~Hz}), 160.0$, 151.78, $149.2(\mathrm{t}, J=3.7 \mathrm{~Hz}), 134.3,131.5,130.9,129.7(\mathrm{t}, J=2.4 \mathrm{~Hz}), 128.4,125.4,119.7(\mathrm{t}$, $J=3.3 \mathrm{~Hz}), 114.0(\mathrm{t}, J=31.7 \mathrm{~Hz}), 113.9$ (t, $J=248.4 \mathrm{~Hz}), 113.5,55.3 ;{ }^{19} \mathrm{~F}$ NMR $(376 \mathrm{MHz}$, $\left.\mathrm{CDCl}_{3}\right): \delta-92.4$; HRMS (ESI): exact mass calculated for $[\mathrm{M}+\mathrm{H}]^{+}\left(\mathrm{C}_{20} \mathrm{H}_{15} \mathrm{~F}_{2} \mathrm{~N}_{2} \mathrm{O}_{2} \mathrm{~S}^{+}\right)$requires $\mathrm{m} / \mathrm{z} 385.0817$, found $\mathrm{m} / \mathrm{z} 385.0819$.

4-(5-(1,1-difluoro-2-oxo-2-phenylethyl)imidazo[2,1-b]thiazol-6-yl)benzonitrile (3fc)

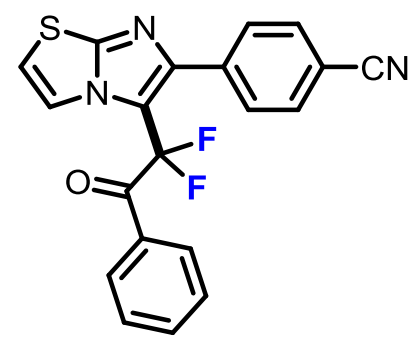

48 h, $66 \mathrm{mg}, 87 \%$ yield, light yellow oil; $\mathrm{R}_{\mathrm{f}}=0.4$ (ethyl acetate $/$ hexane $=20 \%$ ); ${ }^{1} \mathrm{H}$ NMR $\left(400 \mathrm{MHz}, \mathrm{CDCl}_{3}\right): \delta 7.72(\mathrm{t}, J=7.6 \mathrm{~Hz}, 3 \mathrm{H}), 7.64-7.53(\mathrm{~m}, 5 \mathrm{H}), 7.34(\mathrm{t}, J=8.0 \mathrm{~Hz}, 2 \mathrm{H})$, $7.03(\mathrm{~d}, J=4.4 \mathrm{~Hz}, 1 \mathrm{H}) ;{ }^{13} \mathrm{C} \mathrm{NMR}\left(100 \mathrm{MHz}, \mathrm{CDCl}_{3}\right): \delta 186.20(\mathrm{t}, J=31.2 \mathrm{~Hz}), 152.3$, 147.0, 137.6, 134.8, 131.8, 131.2, 130.1, 129.8 (t, $J=2.6 \mathrm{~Hz}), 128.7,119.6(\mathrm{t}, J=3.5 \mathrm{~Hz})$, 118.7, $115.1(\mathrm{t}, J=32.3 \mathrm{~Hz}), 114.6,114.0(\mathrm{t}, J=250.4 \mathrm{~Hz}), 112.18 ;{ }^{19} \mathrm{~F}$ NMR $(376 \mathrm{MHz}$, $\left.\mathrm{CDCl}_{3}\right): \delta$-92.7; HRMS (ESI): exact mass calculated for $[\mathrm{M}+\mathrm{H}]^{+}\left(\mathrm{C}_{20} \mathrm{H}_{12} \mathrm{~F}_{2} \mathrm{~N}_{3} \mathrm{OS}^{+}\right)$requires $\mathrm{m} / \mathrm{z} 380.0664$, found $\mathrm{m} / \mathrm{z} 380.0662$.

\section{2-(6-(4-chlorophenyl)imidazo[2,1-b]thiazol-5-yl)-2,2-difluoro-1-phenylethan-1-} one (3fd)

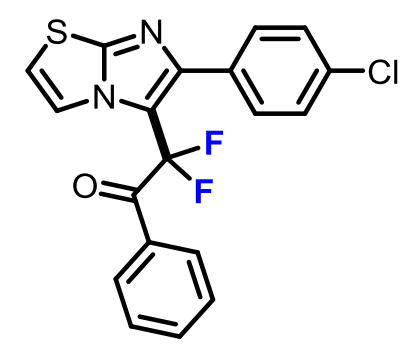

$48 \mathrm{~h}, 52 \mathrm{mg}, 67 \%$ yield, light pink oil, $\mathrm{R}_{\mathrm{f}}=0.4$ (ethyl acetate $/$ hexane $=20 \%$ ); ${ }^{1} \mathrm{H}$ NMR $\left(400 \mathrm{MHz}, \mathrm{CDCl}_{3}\right): \delta 7.72(\mathrm{~d}, J=4.4 \mathrm{~Hz}, 1 \mathrm{H}), 7.68(\mathrm{~d}, J=7.6 \mathrm{~Hz}, 2 \mathrm{H}), 7.52(\mathrm{t}, J=7.2 \mathrm{~Hz}$, $1 \mathrm{H}), 7.35(\mathrm{~d}, J=8.4 \mathrm{~Hz}, 2 \mathrm{H}), 7.29(\mathrm{t}, J=7.6 \mathrm{~Hz}, 4 \mathrm{H}), 6.99(\mathrm{~d}, J=4.4 \mathrm{~Hz}, 1 \mathrm{H}) ;{ }^{13} \mathrm{C} \mathrm{NMR}$ 
$\left(100 \mathrm{MHz}, \mathrm{CDCl}_{3}\right): \delta 186.3,152.0,148.0,134.8,134.6,131.3(\mathrm{t}, J=8.9 \mathrm{~Hz}), 130.9,129.8$, 128.6, 128.3, 119.7, 114.0, $\left.113.9(\mathrm{t}, J=249.5 \mathrm{~Hz}) ;{ }^{19} \mathrm{~F} \mathrm{NMR} \mathrm{(376} \mathrm{MHz,} \mathrm{CDCl}_{3}\right): \delta-92.7$; HRMS (ESI): exact mass calculated for $[\mathrm{M}+\mathrm{H}]^{+}\left(\mathrm{C}_{19} \mathrm{H}_{12} \mathrm{ClF}_{2} \mathrm{~N}_{2} \mathrm{OS}^{+}\right)$requires $m / z$ 389.0321, found $m / z 389.0324$.

2,2-difluoro-1-phenyl-2-(2-phenylbenzo[d]imidazo[2,1-b]thiazol-3-yl)ethan-1-one (3ga)

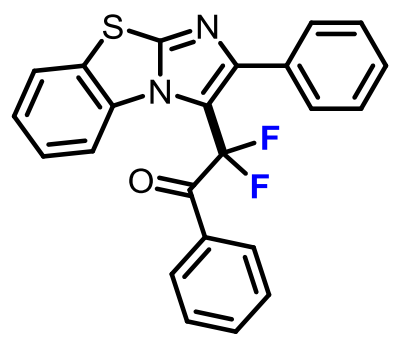

$48 \mathrm{~h}, 63 \mathrm{mg}, 78 \%$ yield, colorless oil, $\mathrm{R}_{\mathrm{f}}=0.4$ (ethyl acetate $/$ hexane $=20 \%$ ); ${ }^{1} \mathrm{H}$ NMR (400 MHz, $\left.\mathrm{CDCl}_{3}\right): \delta 8.07(\mathrm{~d}, J=8.4 \mathrm{~Hz}, 1 \mathrm{H}), 7.77(\mathrm{~d}, J=8.0 \mathrm{~Hz}, 1 \mathrm{H}), 7.64(\mathrm{~d}, J=8.0 \mathrm{~Hz}$, 2H), 7.53-7.41 (m, 3H), 7.28-7.21 (m, 3H), 7.21-7.14 (m, 4H) ; ${ }^{13} \mathrm{C}$ NMR $(100 \mathrm{MHz}$, $\left.\mathrm{CDCl}_{3}\right): \delta 185.8(\mathrm{t}, J=28.9 \mathrm{~Hz}), 151.1,150.6(\mathrm{t}, J=5.3 \mathrm{~Hz}), 134.2,132.8,132.7,131.7$, $130.9,130.2,130.1,129.8,128.7,128.3,127.8,126.7,125.4,124.2,116.0(\mathrm{t}, J=7.0 \mathrm{~Hz})$, $113.6(\mathrm{t}, J=280.0 \mathrm{~Hz}) ;{ }^{19} \mathrm{~F}$ NMR $\left(376 \mathrm{MHz}, \mathrm{CDCl}_{3}\right): \delta-86.2$; HRMS (ESI): exact mass calculated for $[\mathrm{M}+\mathrm{H}]^{+}\left(\mathrm{C}_{23} \mathrm{H}_{15} \mathrm{~F}_{2} \mathrm{~N}_{2} \mathrm{OS}^{+}\right)$requires $\mathrm{m} / \mathrm{z}$ 405.0868, found $\mathrm{m} / \mathrm{z} 405.0869$.

\section{2,2-difluoro-2-(2-(4-methoxyphenyl)benzo[ $d]$ imidazo[2,1-b]thiazol-3-yl)-1-phenylethan-} 1-one (3gb)

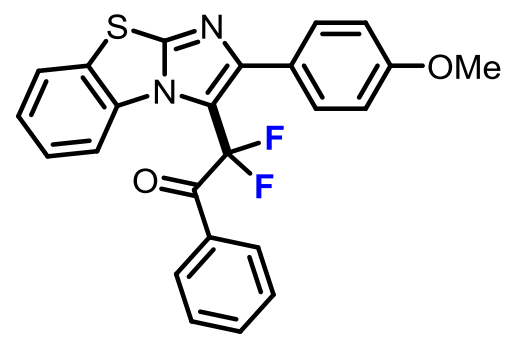

$36 \mathrm{~h}, 75 \mathrm{mg}, 87 \%$ yield, white solid, $\mathrm{R}_{\mathrm{f}}=0.3$ (ethyl acetate /hexane $\left.=25 \%\right) ;{ }^{1} \mathrm{H}$ NMR (400 $\left.\mathrm{MHz}, \mathrm{CDCl}_{3}\right): \delta 8.09(\mathrm{~d}, J=8.4 \mathrm{~Hz}, 1 \mathrm{H}), 7.76(\mathrm{~d}, J=8.0 \mathrm{~Hz}, 1 \mathrm{H}), 7.63(\mathrm{~d}, J=8.0 \mathrm{~Hz}, 2 \mathrm{H})$, 7.51-7.40 (m, 4H), 7.24 (t, $J=8.0 \mathrm{~Hz}, 2 \mathrm{H}), 7.08(\mathrm{~d}, J=8.8 \mathrm{~Hz}, 2 \mathrm{H}), 6.68(\mathrm{~d}, J=8.8 \mathrm{~Hz}, 2 \mathrm{H})$, $3.79(\mathrm{~s}, 3 \mathrm{H}) ;{ }^{13} \mathrm{C} \mathrm{NMR}\left(100 \mathrm{MHz}, \mathrm{CDCl}_{3}\right): \delta 185.7(\mathrm{t}, J=28.6 \mathrm{~Hz}), 160.1,150.5(\mathrm{t}, J=5.6$ 
Hz), 134.1, 132.9, 131.7, 131.4, 130.1, 129.6, 128.2, 126.7, 125.3, 125.1, 124.1, 115.9 (t, $J=$ $6.8 \mathrm{~Hz}), 113.5(\mathrm{t}, J=244.2 \mathrm{~Hz}), 113.31,55.33 ;{ }^{19} \mathrm{~F}$ NMR (376 MHz, $\left.\mathrm{CDCl}_{3}\right): \delta-85.8$; HRMS (ESI): exact mass calculated for $[\mathrm{M}+\mathrm{H}]^{+}\left(\mathrm{C}_{24} \mathrm{H}_{17} \mathrm{~F}_{2} \mathrm{~N}_{2} \mathrm{O}_{2} \mathrm{~S}^{+}\right)$requires $m / z$ 435.0973, found $m / z$ 435.0963.

2-(2-(4-chlorophenyl)benzo[d]imidazo[2,1-b]thiazol-3-yl)-2,2-difluoro-1-phenylethan-1-o ne (3gc)

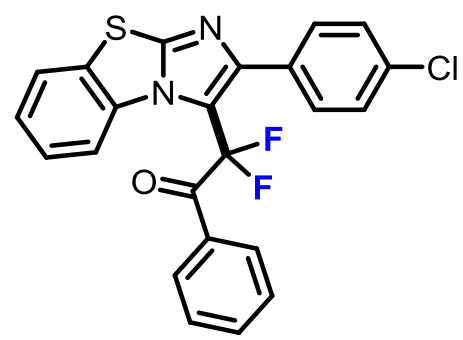

$48 \mathrm{~h}, 64 \mathrm{mg}, 73 \%$ yield, red solid, $\mathrm{R}_{\mathrm{f}}=0.4$ (ethyl acetate $/$ hexane $\left.=20 \%\right) ;{ }^{1} \mathrm{H}$ NMR (400 $\left.\mathrm{MHz}, \mathrm{CDCl}_{3}\right): \delta 8.03(\mathrm{~d}, J=8.0 \mathrm{~Hz}, 1 \mathrm{H}), 7.76(\mathrm{~d}, J=8.0 \mathrm{~Hz}, 1 \mathrm{H}), 7.69(\mathrm{~d}, J=8.0 \mathrm{~Hz}, 2 \mathrm{H})$, $7.51(\mathrm{dd}, J=15.2,7.6 \mathrm{~Hz}, 2 \mathrm{H}), 7.43(\mathrm{t}, J=8.0 \mathrm{~Hz}, 1 \mathrm{H}), 7.31-7.26(\mathrm{~m}, 2 \mathrm{H}), 7.19-7.10(\mathrm{~m}$, $4 \mathrm{H}) ;{ }^{13} \mathrm{C}$ NMR $\left(100 \mathrm{MHz}, \mathrm{CDCl}_{3}\right): \delta 185.8(\mathrm{t}, J=29.3 \mathrm{~Hz}), 151.2,149.2(\mathrm{t}, J=5.1 \mathrm{~Hz})$, 135.0, 134.5, 132.7, 131.6, 131.3, 131.3, 130.2, $129.8(\mathrm{t}, J=2.2 \mathrm{~Hz}), 128.4,128.0,126.8$, 125.5, 124.2, $116.5(\mathrm{t}, J=30.3 \mathrm{~Hz}), 115.9(\mathrm{t}, J=6.8 \mathrm{~Hz}), 113.6(\mathrm{t}, J=245.9 \mathrm{~Hz}) ;{ }^{19} \mathrm{~F} \mathrm{NMR}$ $\left(376 \mathrm{MHz}, \mathrm{CDCl}_{3}\right): \delta-86.7$; HRMS (ESI): exact mass calculated for $[\mathrm{M}+\mathrm{H}]^{+}$ $\left(\mathrm{C}_{23} \mathrm{H}_{14} \mathrm{ClF}_{2} \mathrm{~N}_{2} \mathrm{OS}^{+}\right)$requires $m / z$ 439.0478, found $m / z 439.0482$.

2,2-difluoro-2-(2-(2-fluorophenyl)benzo[d]imidazo[2,1-b]thiazol-3-yl)-1-phenylethan-1-o ne (3gd)

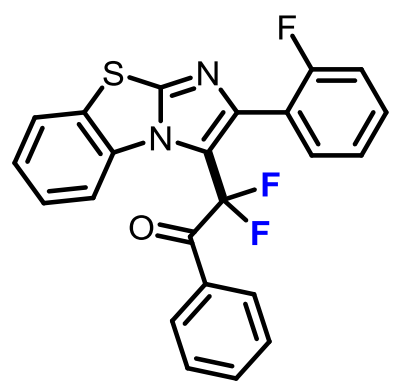

$48 \mathrm{~h}, 44 \mathrm{mg}, 52 \%$ yield, light yellow oil, $\mathrm{R}_{\mathrm{f}}=0.4$ (ethyl acetate $/$ hexane $=20 \%$ ); ${ }^{1} \mathrm{H}$ NMR $\left(400 \mathrm{MHz}, \mathrm{CDCl}_{3}\right): \delta 8.03(\mathrm{~d}, J=8.0 \mathrm{~Hz}, 1 \mathrm{H}), 7.76(\mathrm{~d}, J=8.0 \mathrm{~Hz}, 1 \mathrm{H}), 7.69(\mathrm{~d}, J=8.0 \mathrm{~Hz}$, 
2H), $7.51(\mathrm{dd}, J=15.2,7.6 \mathrm{~Hz}, 2 \mathrm{H}), 7.43(\mathrm{t}, J=8.0 \mathrm{~Hz}, 1 \mathrm{H}), 7.31-7.26(\mathrm{~m}, 2 \mathrm{H}), 7.19-7.10$ $(\mathrm{m}, 4 \mathrm{H}) ;{ }^{13} \mathrm{C} \mathrm{NMR}\left(100 \mathrm{MHz}, \mathrm{CDCl}_{3}\right): \delta 186.2(\mathrm{t}, J=30.4 \mathrm{~Hz}), 161.3,158.8,151.1,143.2$, 134.5, 132.6, 132.3, 131.6, 130.8 (d, $J=8.4 \mathrm{~Hz}), 130.2,129.9$ (t, $J=2.5 \mathrm{~Hz}), 128.5,126.6$, 125.5, 124.2, $123.7(\mathrm{~d}, J=3.7 \mathrm{~Hz}), 121.2(\mathrm{~d}, J=15.0 \mathrm{~Hz}), 118.0(\mathrm{t}, J=30.4 \mathrm{~Hz}), 115.9(\mathrm{t}, J$ $=6.4 \mathrm{~Hz}), 115.4,115.1,114.1(\mathrm{t}, J=247.7 \mathrm{~Hz}) . ;{ }^{19} \mathrm{~F} \mathrm{NMR}\left(376 \mathrm{MHz}, \mathrm{CDCl}_{3}\right): \delta$-89.4, $-113.1(\mathrm{t}, J=4.9 \mathrm{~Hz})$; HRMS (ESI): exact mass calculated for $[\mathrm{M}+\mathrm{H}]^{+}\left(\mathrm{C}_{23} \mathrm{H}_{14} \mathrm{~F}_{3} \mathrm{~N}_{2} \mathrm{OS}^{+}\right)$ requires $m / z$ 423.0773, found $m / z$ 423.0776.

\section{2,2-difluoro-1-phenyl-2-(2-phenylimidazo[1,2-a]pyridin-3-yl)ethan-1-one (3ha)}

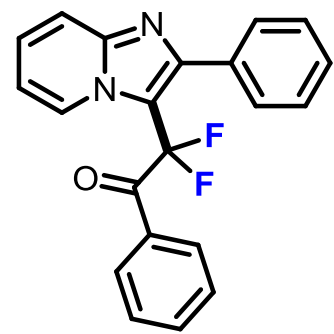

$48 \mathrm{~h}, 53 \mathrm{mg}, 77 \%$ yield, colorless oil, $\mathrm{R}_{\mathrm{f}}=0.4$ (ethyl acetate $/$ hexane $=20 \%$ ); ${ }^{1} \mathrm{H}$ NMR (400 MHz, $\left.\mathrm{CDCl}_{3}\right): \delta 8.52(\mathrm{~d}, J=6.8 \mathrm{~Hz}, 1 \mathrm{H}), 7.70(\mathrm{~d}, J=8.8 \mathrm{~Hz}, 1 \mathrm{H}), 7.65(\mathrm{~d}, J=8.0 \mathrm{~Hz}$, 2H), 7.47 (t, $J=7.6 \mathrm{~Hz}, 1 \mathrm{H}), 7.42-7.25(\mathrm{~m}, 6 \mathrm{H}), 7.22(\mathrm{t}, J=7.6 \mathrm{~Hz}, 2 \mathrm{H}), 6.98$ (t, $J=6.8 \mathrm{~Hz}$, $1 \mathrm{H}) ;{ }^{13} \mathrm{C} \mathrm{NMR}\left(100 \mathrm{MHz}, \mathrm{CDCl}_{3}\right): \delta 186.0(\mathrm{t}, J=30.1 \mathrm{~Hz}), 148.4,146.4,134.7,134.32$, $133.1,131.4,130.2,129.8,129.8,129.7,129.0,128.8,128.37,127.9,126.9,126.5(\mathrm{t}, J=4.8$ $\mathrm{Hz}), 117.9,114.5(\mathrm{t}, J=248.0 \mathrm{~Hz}), 113.7,112.3(\mathrm{t}, J=31.1 \mathrm{~Hz}) ;{ }^{19} \mathrm{~F}$ NMR $(376 \mathrm{MHz}$, $\left.\mathrm{CDCl}_{3}\right): \delta$-94.2 ; HRMS (ESI): exact mass calculated for $[\mathrm{M}+\mathrm{H}]^{+}\left(\mathrm{C}_{21} \mathrm{H}_{15} \mathrm{~F}_{2} \mathrm{~N}_{2} \mathrm{O}^{+}\right)$requires $m / z$ 349.1147, found $m / z$ 349.1154.

2,2-difluoro-2-(2-phenylimidazo[1,2-a]pyridin-3-yl)-1-(p-tolyl)ethan-1-one (3hb)

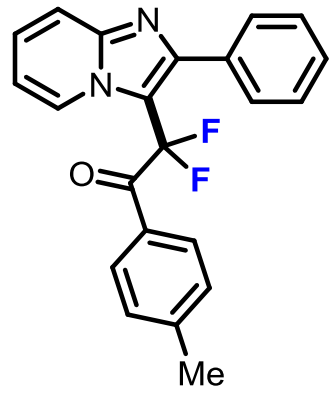


$48 \mathrm{~h}, 53 \mathrm{mg}, 73 \%$ yield, yellow oil, $\mathrm{R}_{\mathrm{f}}=0.4$ (ethyl acetate $/$ hexane $\left.=20 \%\right) ;{ }^{1} \mathrm{H}$ NMR $(400$ $\left.\mathrm{MHz}, \mathrm{CDCl}_{3}\right): \delta 8.49(\mathrm{~d}, J=7.2 \mathrm{~Hz}, 1 \mathrm{H}), 7.69(\mathrm{~d}, J=9.2 \mathrm{~Hz}, 1 \mathrm{H}), 7.57(\mathrm{~d}, J=8.0 \mathrm{~Hz}, 2 \mathrm{H})$, $7.44(\mathrm{~d}, J=6.8 \mathrm{~Hz}, 2 \mathrm{H}), 7.39-7.29(\mathrm{~m}, 4 \mathrm{H}), 7.02(\mathrm{~d}, J=8.0 \mathrm{~Hz}, 2 \mathrm{H}), 6.96(\mathrm{t}, J=6.8 \mathrm{~Hz}$, 1H), 2.32, (s, 3H) ; $\left.{ }^{13} \mathrm{C} \mathrm{NMR} \mathrm{(100} \mathrm{MHz,} \mathrm{CDCl}_{3}\right): \delta 185.7(\mathrm{t}, J=30.3 \mathrm{~Hz}), 148.2,146.4$, $145.6,133.3,130.2,130.05(\mathrm{t}, J=2.6 \mathrm{~Hz}), 129.1,128.9,128.7,127.9,126.7,126.5(\mathrm{t}, J=4.8$ $\mathrm{Hz}), 117.9,114.6(\mathrm{t}, J=248.9 \mathrm{~Hz}), 113.5,21.72 ;{ }^{19} \mathrm{~F} \mathrm{NMR}\left(376 \mathrm{MHz}, \mathrm{CDCl}_{3}\right): \delta-94.4$; HRMS (ESI): exact mass calculated for $[\mathrm{M}+\mathrm{H}]^{+}\left(\mathrm{C}_{22} \mathrm{H}_{17} \mathrm{~F}_{2} \mathrm{~N}_{2} \mathrm{O}^{+}\right)$requires $\mathrm{m} / \mathrm{z}$ 363.1303, found $m / z 363.1304$.

\section{2,2-difluoro-1-(4-methoxyphenyl)-2-(2-phenylimidazo[1,2-a]pyridin-3-yl)ethan-1-one} (3hc)

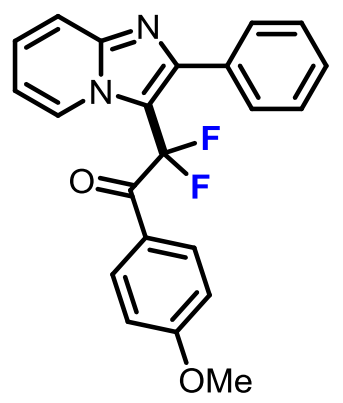

48 h, $64 \mathrm{mg}, 85 \%$ yield; colorless oil, $\mathrm{R}_{\mathrm{f}}=0.3$ (ethyl acetate $/$ hexane $=25 \%$ ); ${ }^{1} \mathrm{H}$ NMR $\left(400 \mathrm{MHz}, \mathrm{CDCl}_{3}\right): \delta 8.49(\mathrm{~d}, J=6.8 \mathrm{~Hz}, 1 \mathrm{H}), 7.68(\mathrm{t}, J=9.6 \mathrm{~Hz}, 3 \mathrm{H}), 7.45(\mathrm{~d}, J=6.8 \mathrm{~Hz}$, 2H), 7.39-7.28 (m, 4H), $6.95(\mathrm{t}, J=6.8 \mathrm{~Hz}, 1 \mathrm{H}), 6.69(\mathrm{~d}, J=8.8 \mathrm{~Hz}, 2 \mathrm{H}), 3.80(\mathrm{~s}, 3 \mathrm{H}) ;{ }^{13} \mathrm{C}$ NMR (100 MHz, $\left.\mathrm{CDCl}_{3}\right): \delta 184.6(\mathrm{t}, J=30.1 \mathrm{~Hz}), 164.5,148.1,146.3,133.4,132.5(\mathrm{~d}, J=$ $2.6 \mathrm{~Hz}), 130.2,128.7,127.9,126.7,126.6(\mathrm{t}, J=4.8 \mathrm{~Hz}), 124.3,117.8,113.7,114.7(\mathrm{t}, J=$ 248.6 Hz), 113.5, $55.5 ;{ }^{19} \mathrm{~F}$ NMR (376 $\left.\mathrm{MHz}, \mathrm{CDCl}_{3}\right): \delta-94.2$; HRMS (ESI): exact mass calculated for $[\mathrm{M}+\mathrm{H}]^{+}\left(\mathrm{C}_{22} \mathrm{H}_{17} \mathrm{~F}_{2} \mathrm{~N}_{2} \mathrm{O}_{2}{ }^{+}\right)$requires $m / z$ 379.1253, found $m / z$ 379.1236. 


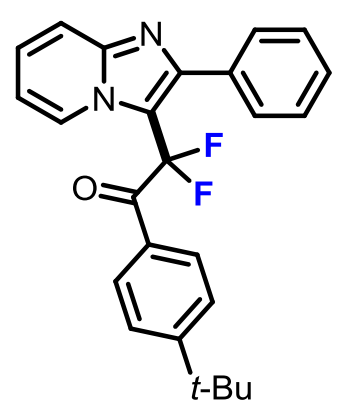

$48 \mathrm{~h}, 70 \mathrm{mg}, 87 \%$ yield, colorless oil, $\mathrm{R}_{\mathrm{f}}=0.4$ (ethyl acetate $/$ hexane $=20 \%$ ); ${ }^{1} \mathrm{H}$ NMR $\left(400 \mathrm{MHz}, \mathrm{CDCl}_{3}\right): \delta 8.50(\mathrm{~d}, J=7.2 \mathrm{~Hz}, 1 \mathrm{H}), 7.70(\mathrm{~d}, J=8.8 \mathrm{~Hz}, 1 \mathrm{H}), 7.60(\mathrm{~d}, J=8.0 \mathrm{~Hz}$, 2H), $7.40(\mathrm{~d}, J=7.2 \mathrm{~Hz}, 2 \mathrm{H}), 7.36-7.28(\mathrm{~m}, 4 \mathrm{H}), 7.23(\mathrm{~d}, J=8.4 \mathrm{~Hz}, 2 \mathrm{H}), 6.96$ (t, $J=6.8 \mathrm{~Hz}$, 1H), $1.26(\mathrm{~s}, 9 \mathrm{H}) ;{ }^{13} \mathrm{C} \mathrm{NMR}\left(100 \mathrm{MHz}, \mathrm{CDCl}_{3}\right): \delta 185.6(\mathrm{t}, J=30.1 \mathrm{~Hz}), 158.4,148.3,146.4$, $133.3,130.1(\mathrm{~d}, J=9.9 \mathrm{~Hz}), 130.0(\mathrm{~d}, J=14.7 \mathrm{~Hz}), 128.8,128.7,127.9,126.8,126.5(\mathrm{t}, J=$ $4.8 \mathrm{~Hz}), 125.4,117.9,113.6,113.6(\mathrm{t}, J=248.4 \mathrm{~Hz}), 112.5(\mathrm{t}, J=31.4 \mathrm{~Hz}), 35.2,30.9 ;{ }^{19} \mathrm{~F}$ NMR (376 MHz, $\left.\mathrm{CDCl}_{3}\right): \delta$-94.4 ; HRMS (ESI): exact mass calculated for $[\mathrm{M}+\mathrm{H}]^{+}$ $\left(\mathrm{C}_{25} \mathrm{H}_{22} \mathrm{~F}_{2} \mathrm{~N}_{2} \mathrm{O}^{+}\right)$requires $m / z$ 405.1773, found $m / z$ 405.1783.

\section{2,2-difluoro-1-(4-fluorophenyl)-2-(2-phenylimidazo[1,2-a]pyridin-3-yl)ethan-1-one (3he)}

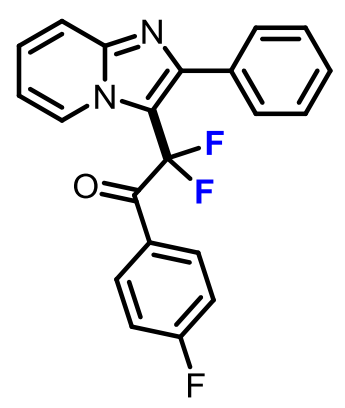

$66 \mathrm{~h}, 57 \mathrm{mg}, 78 \%$ yield, light yellow oil, $\mathrm{R}_{\mathrm{f}}=0.4$ (ethyl acetate $/$ hexane $=20 \%$ ); ${ }^{1} \mathrm{H}$ NMR $\left(400 \mathrm{MHz}, \mathrm{CDCl}_{3}\right): \delta 8.52(\mathrm{~d}, J=5.2 \mathrm{~Hz}, 1 \mathrm{H}), 7.72-7.67(\mathrm{~m}, 3 \mathrm{H}), 7.46-7.22(\mathrm{~m}, 6 \mathrm{H}), 6.99(\mathrm{~d}$, $J=5.6 \mathrm{~Hz}, 1 \mathrm{H}), 6.89-6.86(\mathrm{~m}, 2 \mathrm{H}) ;{ }^{13} \mathrm{C} \mathrm{NMR}\left(100 \mathrm{MHz}, \mathrm{CDCl}_{3}\right): \delta 184.5(\mathrm{t}, J=29.9 \mathrm{~Hz})$, 167.5, 165.0, 148.4, 146.5, 133.1, 132.7 (d, $J=9.5 \mathrm{~Hz}), 130.2$, 128.9, 127.9, 127.1, 126.5, 118.0, 115.8, 115.5, $114.4(\mathrm{t}, J=247.6 \mathrm{~Hz}), 113.7,112.1(\mathrm{t}, J=26.8 \mathrm{~Hz}) ;{ }^{19} \mathrm{~F}$ NMR $(376$ $\left.\mathrm{MHz}, \mathrm{CDCl}_{3}\right): \delta$-94.0, -101.7--101.8 (m) ; HRMS (ESI): exact mass calculated for $[\mathrm{M}+\mathrm{H}]^{+}$ $\left(\mathrm{C}_{21} \mathrm{H}_{14} \mathrm{~F}_{3} \mathrm{~N}_{2} \mathrm{O}^{+}\right)$requires $m / z$ 367.1053, found $m / z$ 367.1055. 


\section{2,2-difluoro-1-(naphthalen-2-yl)-2-(2-phenylimidazo[1,2-a]pyridin-3-yl)ethan-1-}

one (3hf)

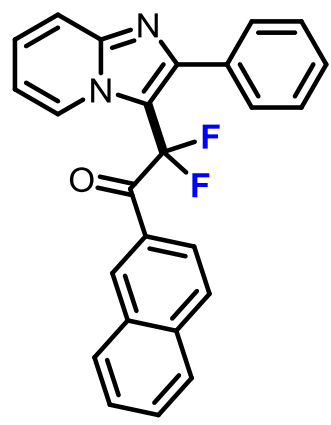

$72 \mathrm{~h}, 55 \mathrm{mg}, 69 \%$ yield, light yellow oil, $\mathrm{R}_{\mathrm{f}}=0.4$ (ethyl acetate $/$ hexane $=20 \%$ ); ${ }^{1} \mathrm{H}$ NMR $\left(400 \mathrm{MHz}, \mathrm{CDCl}_{3}\right): \delta 8.56(\mathrm{~d}, J=7.2 \mathrm{~Hz}, 1 \mathrm{H}), 8.17(\mathrm{~s}, 1 \mathrm{H}), 7.80-7.73(\mathrm{~m}, 2 \mathrm{H}), 7.69(\mathrm{~d}, J=$ $8.8 \mathrm{~Hz}, 2 \mathrm{H}), 7.59-7.45(\mathrm{~m}, 5 \mathrm{H}), 7.44-7.33(\mathrm{~m}, 4 \mathrm{H}), 6.99(\mathrm{t}, J=7.2 \mathrm{~Hz}, 1 \mathrm{H}) ;{ }^{13} \mathrm{C} \mathrm{NMR}(100$ $\left.\mathrm{MHz}, \mathrm{CDCl}_{3}\right): \delta 186.1(\mathrm{t}, J=30.6 \mathrm{~Hz}), 148.2,146.5,135.9,133.5,132.8(\mathrm{t}, J=3.7 \mathrm{~Hz})$, $131.9,130.9,130.4,130.0,129.5,128.9,128.5,128.3,128.0,127.6,126.9,126.6(\mathrm{t}, J=4.4$ $\mathrm{Hz}), 124.5,117.9,114.7(\mathrm{t}, J=249.6 \mathrm{~Hz}), 113.7 ;{ }^{19} \mathrm{~F}$ NMR $\left(376 \mathrm{MHz}, \mathrm{CDCl}_{3}\right): \delta-94.2$; HRMS (ESI): exact mass calculated for $[\mathrm{M}+\mathrm{H}]^{+}\left(\mathrm{C}_{25} \mathrm{H}_{17} \mathrm{~F}_{2} \mathrm{~N}_{2} \mathrm{O}^{+}\right)$requires $\mathrm{m} / \mathrm{z}$ 399.1303, found $m / z$ 399.1300.

\section{2,2-difluoro-2-(2-phenylimidazo[1,2-a]pyridin-3-yl)-1-(thiophen-2-yl)ethan-1-one (3hg)}

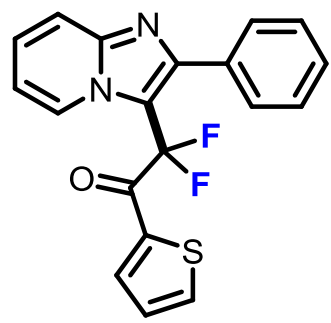

$72 \mathrm{~h}, 56 \mathrm{mg}, 79 \%$ yield, light green oil, $\mathrm{R}_{\mathrm{f}}=0.5$ (ethyl acetate $/$ hexane $=20 \%$ ); ${ }^{1} \mathrm{H}$ NMR (400 MHz, $\left.\mathrm{CDCl}_{3}\right): \delta 8.54(\mathrm{~d}, J=6.8 \mathrm{~Hz}, 1 \mathrm{H}), 7.70(\mathrm{~d}, J=8.8 \mathrm{~Hz}, 1 \mathrm{H}), 7.65(\mathrm{~d}, J=4.8 \mathrm{~Hz}$, $1 \mathrm{H}), 7.48(\mathrm{~d}, J=7.2 \mathrm{~Hz}, 2 \mathrm{H}), 7.43(\mathrm{~d}, J=2.8 \mathrm{~Hz}, 1 \mathrm{H}), 7.41-7.33(\mathrm{~m}, 4 \mathrm{H}), 6.98(\mathrm{~d}, J=6.8 \mathrm{~Hz}$, 1H), 6.96-6.92 (m, 1H) ; $\left.{ }^{13} \mathrm{C} \mathrm{NMR} \mathrm{(100} \mathrm{MHz,} \mathrm{CDCl}_{3}\right): \delta 179.6(\mathrm{t}, J=32.5 \mathrm{~Hz}), 148.5,146.4$, 137.6, 136.8, $135.8(\mathrm{t}, J=4.0 \mathrm{~Hz}), 133.4,130.2,128.8,128.5,127.9,126.9,126.6(\mathrm{t}, J=4.6$ $\mathrm{Hz}), 117.8,114.2(\mathrm{t}, J=249.5 \mathrm{~Hz}), 113.6,111.9(\mathrm{t}, J=25.5 \mathrm{~Hz}) ;{ }^{19} \mathrm{~F}$ NMR $(376 \mathrm{MHz}$, 
$\left.\mathrm{CDCl}_{3}\right): \delta$-95.5; HRMS (ESI): exact mass calculated for $[\mathrm{M}+\mathrm{H}]^{+}\left(\mathrm{C}_{19} \mathrm{H}_{13} \mathrm{~F}_{2} \mathrm{~N}_{2} \mathrm{OS}^{+}\right)$requires $m / z$ 355.0711, found $m / z 355.0710$.

1-(benzo[d][1,3]dioxol-5-yl)-2,2-difluoro-2-(2-phenylimidazo[1,2-a]pyridin-3-yl)ethan-1one (3hh)

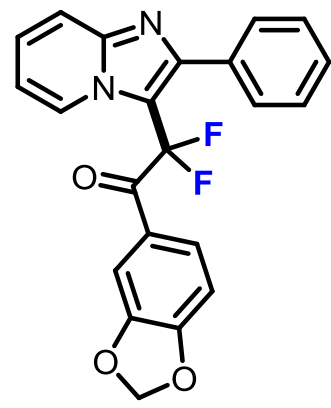

$66 \mathrm{~h}, 58 \mathrm{mg}, 74 \%$ yield, light yellow oil, $\mathrm{R}_{\mathrm{f}}=0.3$ (ethyl acetate $/$ hexane $=25 \%$ ); ${ }^{1} \mathrm{H}$ NMR (400 MHz, $\left.\mathrm{CDCl}_{3}\right): \delta 8.48(\mathrm{~d}, J=7.2 \mathrm{~Hz}, 1 \mathrm{H}), 7.70(\mathrm{~d}, J=8.8 \mathrm{~Hz}, 1 \mathrm{H}), 7.42(\mathrm{~d}, J=7.2$ $\mathrm{Hz}, 2 \mathrm{H}), 7.34(\mathrm{dt}, J=14.0,6.4 \mathrm{~Hz}, 5 \mathrm{H}), 7.11(\mathrm{~s}, 1 \mathrm{H}), 6.96(\mathrm{t}, J=6.8 \mathrm{~Hz}, 1 \mathrm{H}), 6.59(\mathrm{~d}, J=8.4$ $\mathrm{Hz}, 1 \mathrm{H}), 5.97(\mathrm{~s}, 2 \mathrm{H}) ;{ }^{13} \mathrm{C} \mathrm{NMR}\left(100 \mathrm{MHz}, \mathrm{CDCl}_{3}\right): \delta 184.1(\mathrm{t}, J=29.9 \mathrm{~Hz}), 152.9,148.2(\mathrm{t}$, $J=2.9 \mathrm{~Hz}), 147.9,146.3,133.3,130.2,128.7,127.9,127.2(\mathrm{t}, J=3.3 \mathrm{~Hz}), 126.8,126.5(\mathrm{t}, J$ $=4.8 \mathrm{~Hz}), 125.9,117.8,113.6,114.5(\mathrm{t}, J=248.3 \mathrm{~Hz}), 112.5(\mathrm{t}, J=31.5 \mathrm{~Hz}), 109.3(\mathrm{t}, J=$ 2.1 Hz), 107.8, $102.0 ;{ }^{19} \mathrm{~F}$ NMR (376 MHz, $\left.\mathrm{CDCl}_{3}\right): \delta-93.7$; HRMS (ESI): exact mass calculated for $[\mathrm{M}+\mathrm{H}]^{+}\left(\mathrm{C}_{22} \mathrm{H}_{15} \mathrm{~F}_{2} \mathrm{~N}_{2} \mathrm{O}_{3}{ }^{+}\right)$requires $m / z$ 393.1045, found $m / z$ 393.1049.

1-(4-(tert-butyl)phenyl)-2,2-difluoro-2-(6-phenylimidazo[2,1-b]thiazol-5-yl)ethan-1-one (3ia)

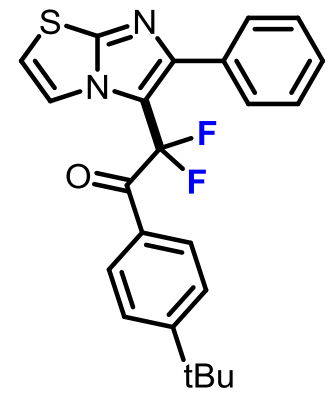

$63 \mathrm{~h}, 71 \mathrm{mg}, 87 \%$ yield, light yellow oil, $\mathrm{R}_{\mathrm{f}}=0.5$ (ethyl acetate $/$ hexane $=20 \%$ ); ${ }^{1} \mathrm{H}$ NMR (400 MHz, $\left.\mathrm{CDCl}_{3}\right): \delta 7.72(\mathrm{~d}, J=4.4 \mathrm{~Hz}, 1 \mathrm{H}), 7.60(\mathrm{~d}, J=7.6 \mathrm{~Hz}, 2 \mathrm{H}), 7.42(\mathrm{~d}, J=5.6 \mathrm{~Hz}$, 
2H), $7.31(\mathrm{~d}, J=7.2 \mathrm{~Hz}, 3 \mathrm{H}), 7.25(\mathrm{~d}, J=8.4 \mathrm{~Hz}, 2 \mathrm{H}), 6.96(\mathrm{~d}, J=4.4 \mathrm{~Hz}, 1 \mathrm{H}), 1.27(\mathrm{~s}, 9 \mathrm{H})$;

${ }^{13} \mathrm{C}$ NMR (100 MHz, $\left.\mathrm{CDCl}_{3}\right): \delta 186.0(\mathrm{t}, J=30.4 \mathrm{~Hz}), 158.5,151.8,149.2,133.1,129.8$, 129.6, 128.8, 128.6, 128.0, 125.4, 119.8, $114.6(\mathrm{t}, J=32.1 \mathrm{~Hz}), 114.0(\mathrm{t}, J=248.7 \mathrm{~Hz}), 113.6$, $35.2,30.9 ;{ }^{19} \mathrm{~F}$ NMR $\left(376 \mathrm{MHz}, \mathrm{CDCl}_{3}\right): \delta-92.9$; HRMS (ESI): exact mass calculated for $[\mathrm{M}+\mathrm{H}]^{+}\left(\mathrm{C}_{23} \mathrm{H}_{21} \mathrm{~F}_{2} \mathrm{~N}_{2} \mathrm{OS}^{+}\right)$requires $m / z$ 411.1337, found $\mathrm{m} / z 411.1339$.

\section{2,2-difluoro-1-(4-methoxyphenyl)-2-(6-phenylimidazo[2,1-b]thiazol-5-yl)ethan-1-one} (3ib)

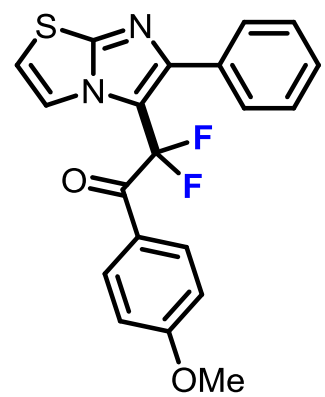

$72 \mathrm{~h}, 64 \mathrm{mg}, 83 \%$ yield, yellow oil, $\mathrm{R}_{\mathrm{f}}=0.3$ (ethyl acetate $/$ hexane $\left.=25 \%\right) ;{ }^{1} \mathrm{H}$ NMR $(400$ $\left.\mathrm{MHz}, \mathrm{CDCl}_{3}\right): \delta 7.64(\mathrm{~d}, J=4.4 \mathrm{~Hz}, 1 \mathrm{H}), 7.59(\mathrm{~d}, J=8.8 \mathrm{~Hz}, 2 \mathrm{H}), 7.42-7.33(\mathrm{~m}, 2 \mathrm{H})$, $7.31-7.23(\mathrm{~m}, 3 \mathrm{H}), 6.88(\mathrm{~d}, J=4.4 \mathrm{~Hz}, 1 \mathrm{H}), 6.64(\mathrm{~d}, J=8.8 \mathrm{~Hz}, 2 \mathrm{H}), 3.74(\mathrm{~s}, 3 \mathrm{H}) ;{ }^{13} \mathrm{C} \mathrm{NMR}$ $\left(100 \mathrm{MHz}, \mathrm{CDCl}_{3}\right): \delta 184.9(\mathrm{t}, J=26.6 \mathrm{~Hz}), 164.5,151.7,149.1,133.1,132.4,130.8,129.7$, $128.8,128.6,128.0,124.3,119.8,114.1(\mathrm{t}, J=248.9 \mathrm{~Hz}), 113.8,113.5,55.5 ;{ }^{19} \mathrm{~F}$ NMR $(376$ $\left.\mathrm{MHz}, \mathrm{CDCl}_{3}\right): \delta-92.8$; HRMS (ESI): exact mass calculated for $[\mathrm{M}+\mathrm{H}]^{+}\left(\mathrm{C}_{20} \mathrm{H}_{15} \mathrm{~F}_{2} \mathrm{~N}_{2} \mathrm{O}_{2} \mathrm{~S}^{+}\right)$ requires $m / z$ 385.0817, found $m / z 385.0821$.

\section{Gram-scale reaction}

Imidazopyridines $1 \mathrm{a}(0.21 \mathrm{~g}, 1.1 \mathrm{mmol}, 1.0 \mathrm{eq})$ was added to a $25 \mathrm{~mL}$ flame-dried flask containing a stirring bar and purged by evacuating the flask and backfilling with argon three times. In the absence of light, tetramethylethylenediamine (315 $\mu \mathrm{L}, 2.2 \mathrm{mmol}, 2.0 \mathrm{eq})$, $\alpha$-bromo difluoroacylarenes $2 \mathbf{a}(0.37 \mathrm{~g}, 1.6 \mathrm{mmol}, 1.5 \mathrm{eq})$ and anhydrous acetonitrile $(11 \mathrm{ml}$, 0.1 M) was added and the flask was sealed. The mixture was then stirred under irradiation from $33 \mathrm{~W}$ CFL. After completion of the reaction, the crude mixture was filtered and the filtrate was concentrated under reduced pressure. The residue was purified by column 
chromatography over silica gel using $20 \%$ ethyl acetate in hexanes as elucent to yield $\mathbf{3 h a}$ $(0.26 \mathrm{~g}, 71 \%)$.

\section{Mechanistic consideration}

\section{Comparative experiment.}

a)
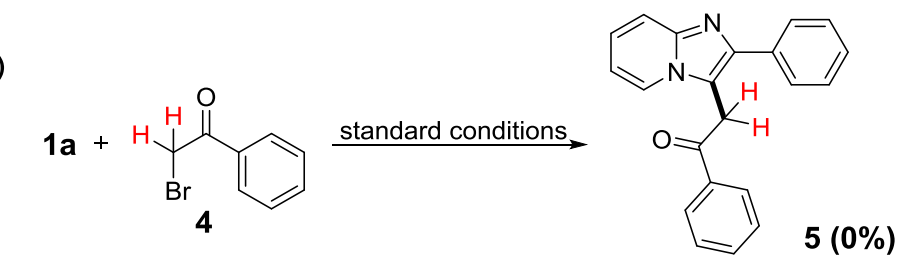

b)
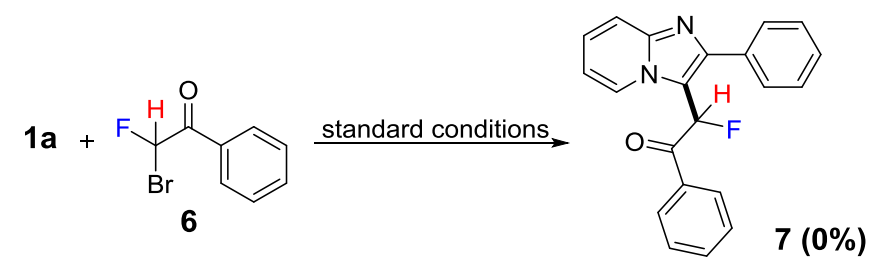

Scheme S1. Comparative experiment.

\section{Radical trapping experiment with 1,1-diphenylethylene.}

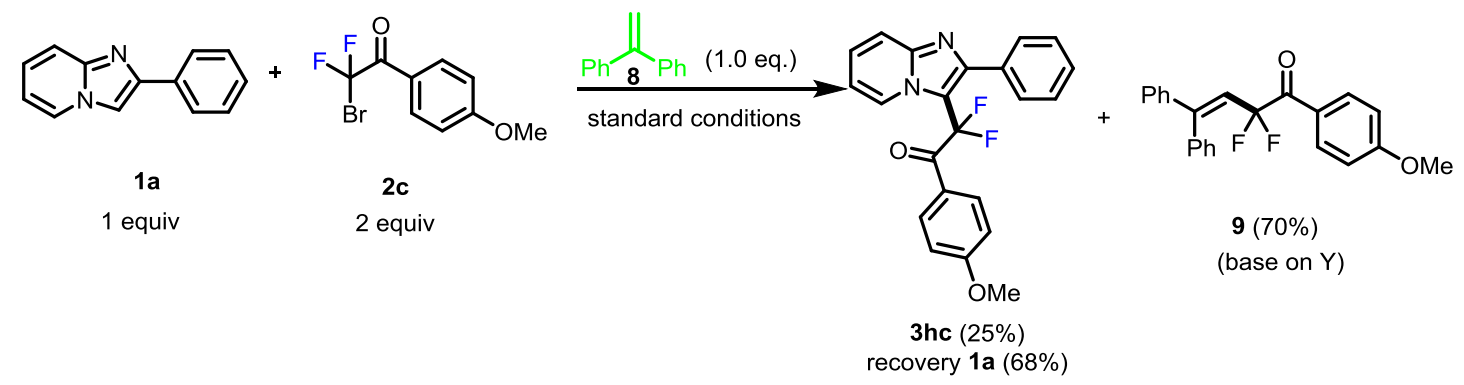

Scheme S2. Trapping experiment in the standard reaction.

To a Schlenk tube charged with a magnetic stirring bar, substrate 1a $(0.2 \mathrm{mmol})$ was added and the Schlenk tube was purged with argon for three times. Subsequently, anhydrous $\mathrm{CH}_{3} \mathrm{CN}(2 \mathrm{ml}), 2 \mathrm{c}(0.3 \mathrm{mmol})$ and 1,1-diphenylethylene $(0.2 \mathrm{mmol})$ were added in turn via syringes. The tightly sealed tube was then irradiated with a $33 \mathrm{~W}$ CFL at room temperature for $48 \mathrm{~h}$. The corresponding difluoroacetylated product 3hc was obtained in $25 \%$ yield and the substrate 1a was recovered in $68 \%$ yield after purification through flash column chromatography on silica gel. In the meanwhile, the $\mathrm{CF}_{2} \mathrm{COAr}$-trapped product 9 was isolated in $70 \%$ yield (based on 1,1-diphenylethylene, Scheme S2), colorless oil, $\mathrm{R}_{\mathrm{f}}=0.4$ $($ Hexane/Ethyl acetate $=95 / 5),{ }^{1} \mathrm{HNMR}\left(400 \mathrm{MHz}, \mathrm{CDCl}_{3}\right): \delta 7.76(\mathrm{~d}, J=8.8 \mathrm{~Hz}, 2 \mathrm{H})$, 
7.24-7.17 (m, 8H), $6.93(\mathrm{~d}, J=7.6 \mathrm{~Hz}, 2 \mathrm{H}), 6.78(\mathrm{~d}, J=8.4 \mathrm{~Hz}, 2 \mathrm{H}), 6.41(\mathrm{t}, J=12.4 \mathrm{~Hz}$, 1H), $3.78(\mathrm{~s}, 3 \mathrm{H}) ;{ }^{13} \mathrm{C}$ NMR (100 MHz, $\left.\mathrm{CDCl}_{3}\right): 164.1,150.9(\mathrm{t}, J=8.6 \mathrm{~Hz}), 140.7,137.2$, $134.2(\mathrm{~d}, J=14.0 \mathrm{~Hz}), 132.3,129.9,129.0,128.5,128.4,127.8,124.9,120.5(\mathrm{t}, J=27.1 \mathrm{~Hz})$, $115.4(\mathrm{t}, J=245.9 \mathrm{~Hz}), 113.6,55.5 ;{ }^{19} \mathrm{~F} \mathrm{NMR}\left(376 \mathrm{MHz}, \mathrm{CDCl}_{3}\right): \delta-87.5$.

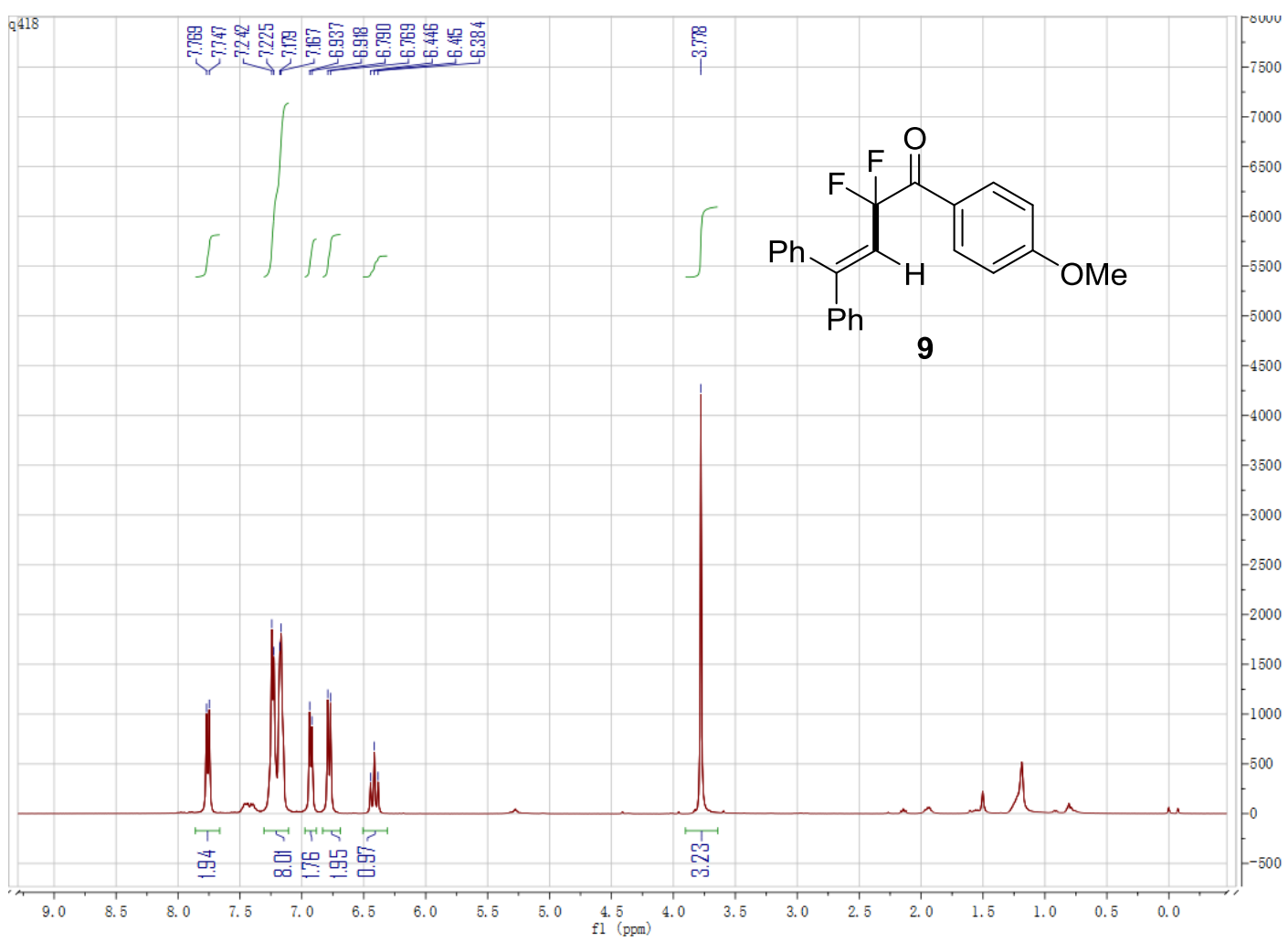



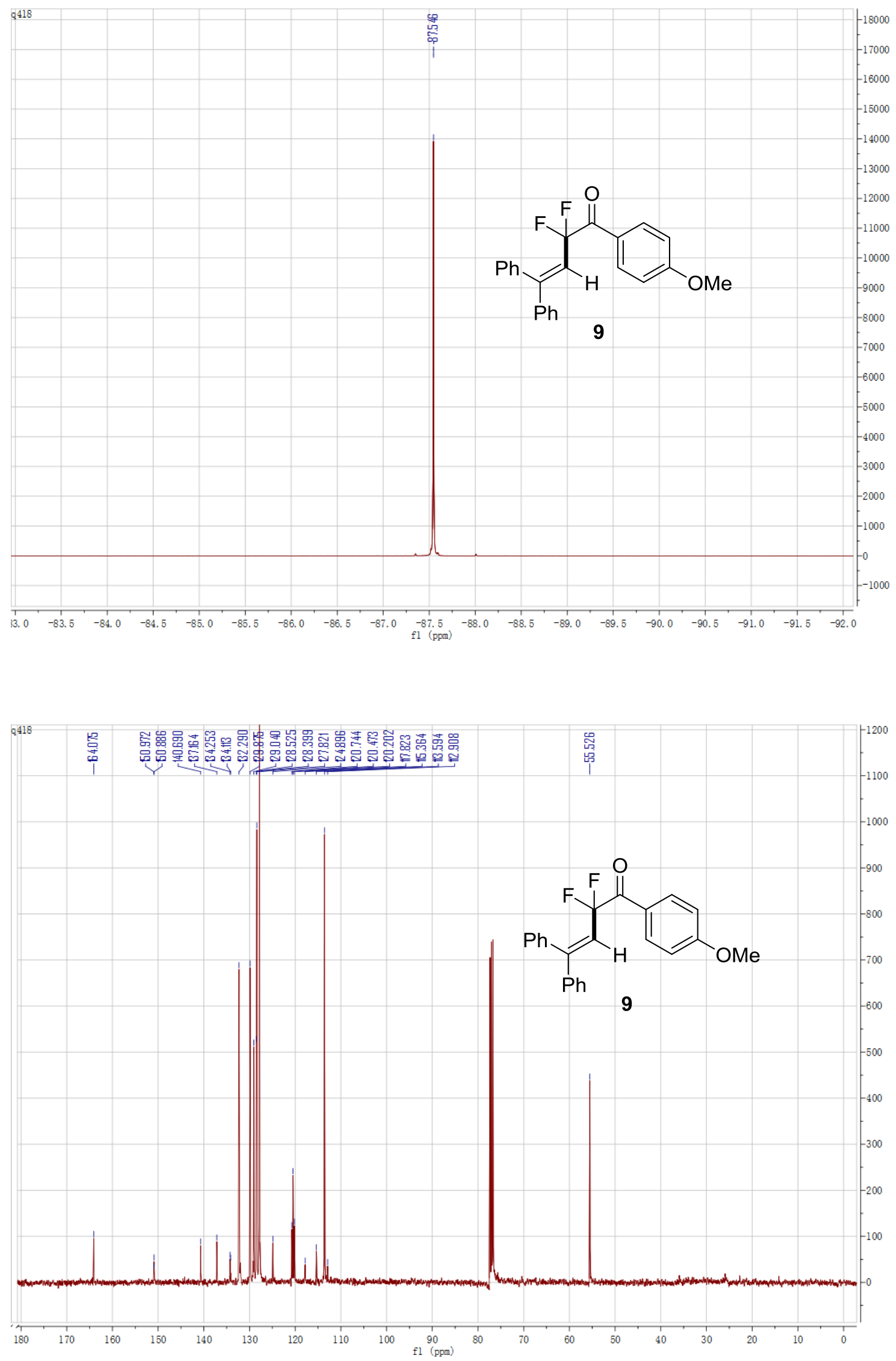

S26 


\section{Trapping experiment with TEMPO free radical}

The most common used for intermediate identification is adding an additional reagent to trap the species. The persistent free radical 2,2,6,6-tetramethylpiperdine 1-oxyl (TEMPO) is usually served as a trapping reagent of carbon-centered radicals, also for intramolecular radical cyclization. Based on above reasons, we applied this radical trapping agent in our reaction to examine its behavior. Under the standard reaction, when 2.0 equivalent of TEMPO (with respect to 1a) was added to the reactions, after long exposure to irradiation (36 h) products 3ha could not be detected (Scheme S3). However, the corresponding alkylfragment of the halides (oxyamination at the alpha-phenacyl positions) were formed (Scheme S3, Figure S1).

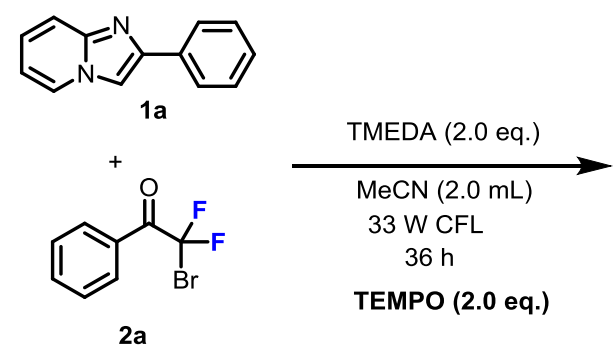

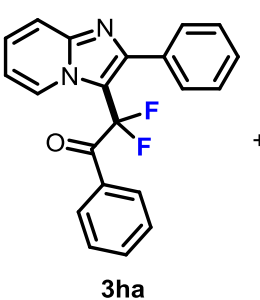

Not observed

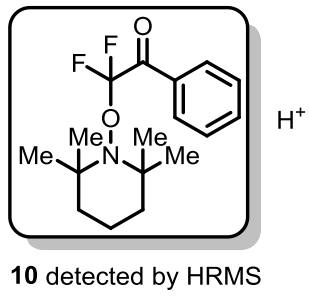

Chemical Formula: $\mathrm{C}_{17} \mathrm{H}_{24} \mathrm{~F}_{2} \mathrm{NO}_{2}{ }^{+}$

Exact Mass: 312.1770

Found: 312.1753

Scheme S3. Trapping experiment in the standard reaction.

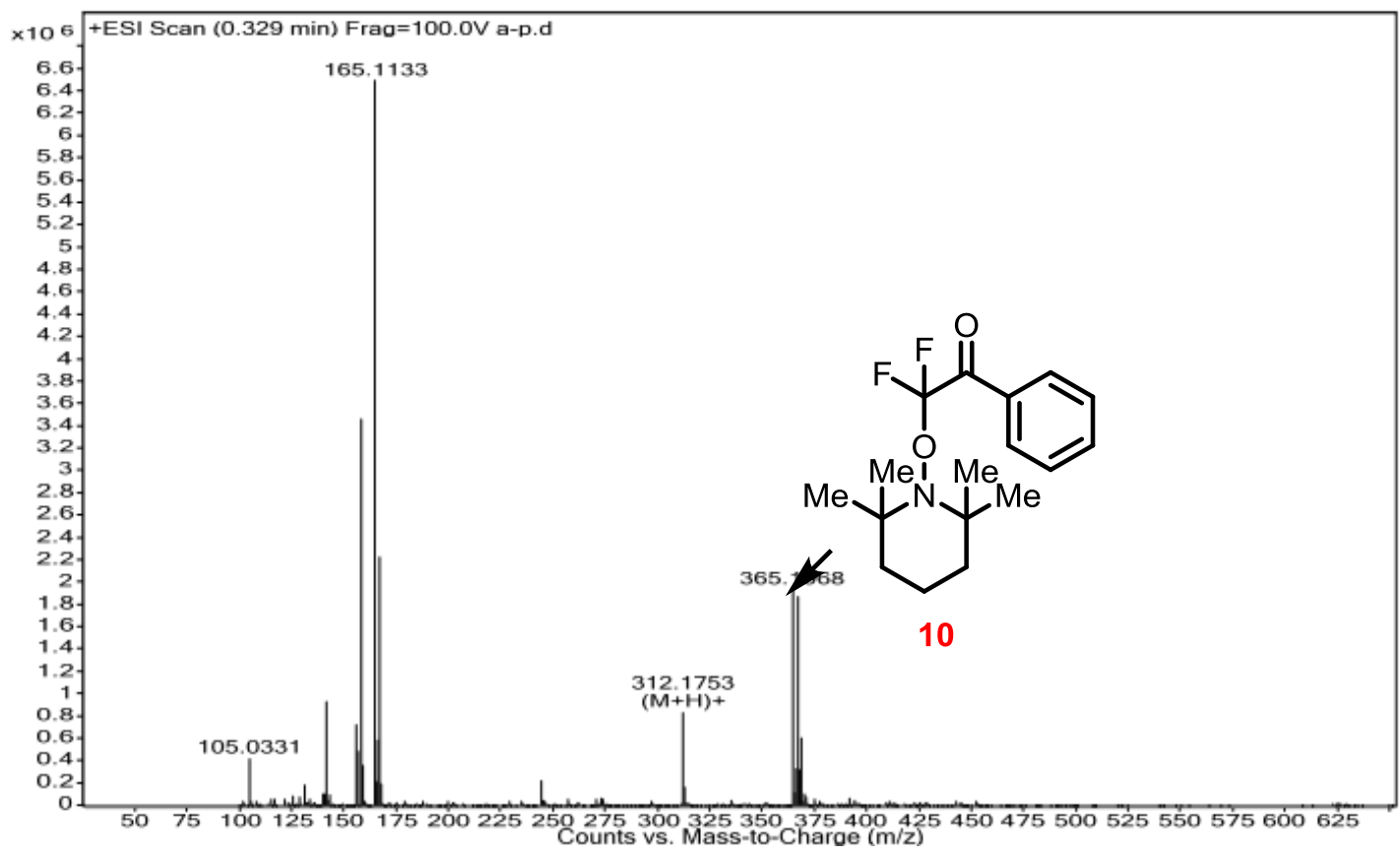

Figure S1: The HRMS data of compound 10. 
This result may suggest the formation of the radical species involved in our reaction conditions, there is another plausible explanation that visible light induced the formation of difluoroacyl radical species $\mathbf{I}$.

\section{UV/Vis Plot}

In Figure S2, we don't really see a bathochromic shift. Upon mixing $\mathbf{1 a}+\mathbf{2 a}$, the new curve is a bit different to the one of $\mathbf{2 a}$ (difluoro-bromo compound) but if we look in the visible range (from $390 \mathrm{~nm}$ ) it is completely superimposable to the one of 1a (imidazopyridines). This observation excludes the formation of an electron donor-acceptor complexes (EDAC) and the following visible light-promoted SET.

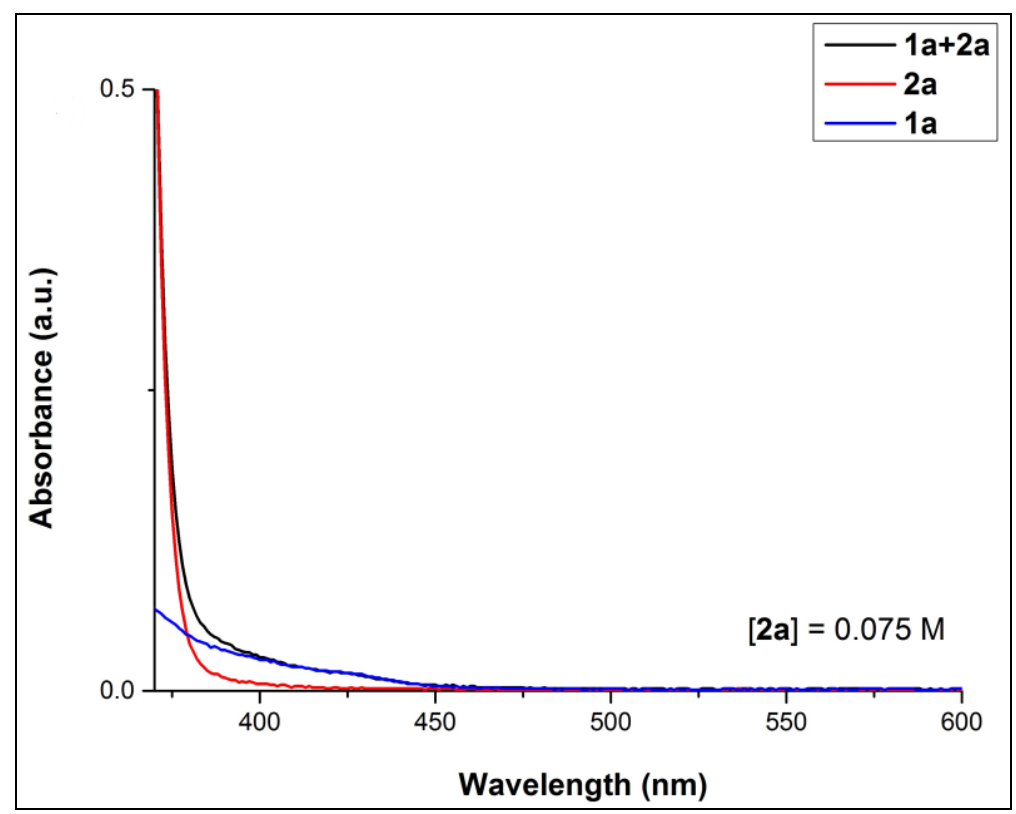

Figure S2. Optical absorption spectra of 2a with 1a (recorded in MeCN in $1 \mathrm{~mm}$ path length quartz cuvettes using a Shimadzu UV-3600 UV-visible spectrophotometer). 


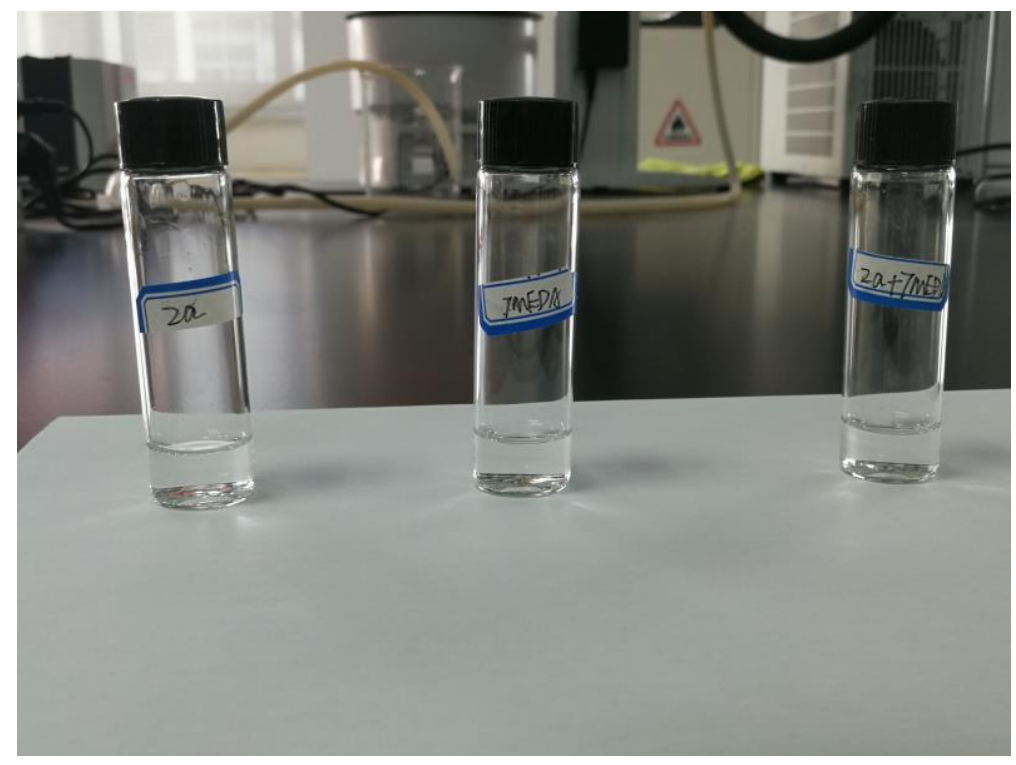

Figure S3. No visual appearance of the separate reaction components and the colored EDA complex.

The UV/Vis absorption spectra of MeCN solutions of TMEDA $(0.067 \mathrm{M}), \mathbf{2 a}(0.1 \mathrm{M})$, and a mixture of 2a and TMEDA are shown in Figure S4. An obvious bathochromic shift can not be observed (the new curve of the solution between 2a and TMEDA is a bit different to the one of $\mathbf{2 a}$, but if we look in the visible range (from $400 \mathrm{~nm}$ ) and ultraviolet zone (below 375 $\mathrm{nm}$ ) it is completely supermposable to the one of 2a.). This observation excludes the formation of electron donor-acceptor complexes (EDAC) and the following visible light-promoted SET. 


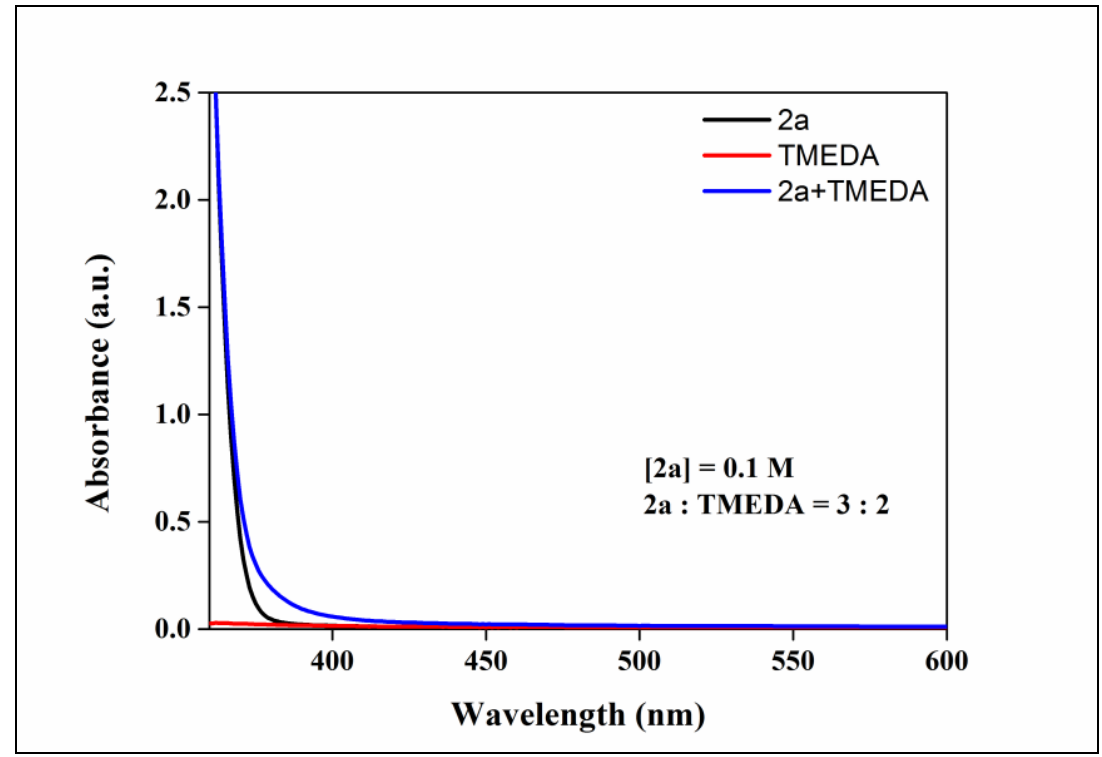

Figure S4. Optical absorption spectra of 2a with TMEDA (recorded in MeCN in $1 \mathrm{~mm}$ path length quartz cuvettes using a Beijing Persee T9 series double beam UV-visible spectrophotometer).

\section{Light-induction requirement}
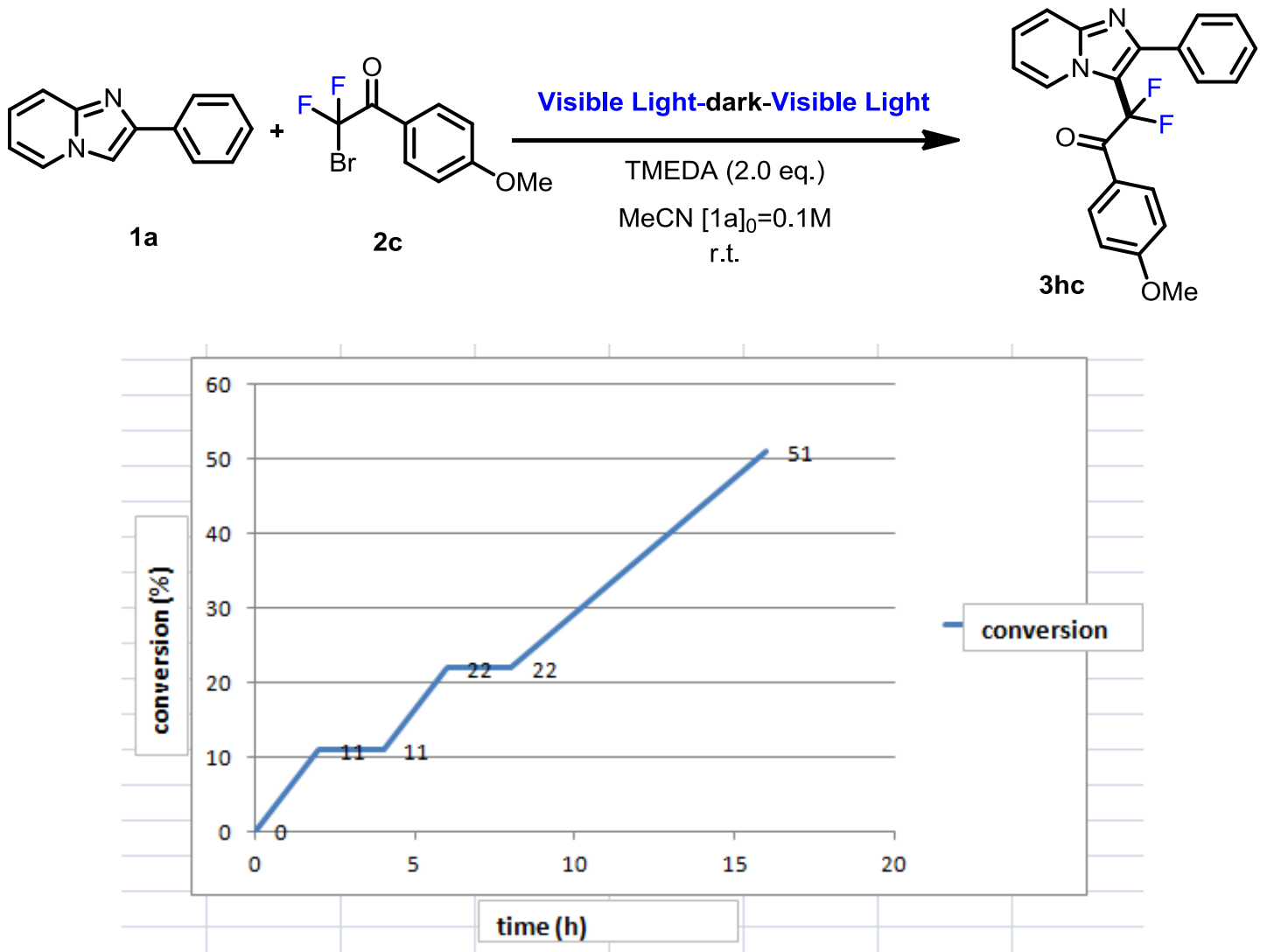

Figure S5. Successive intervals of irradiation and dark periods for reactions. 
The reactions presented in this part are light induced processes. The reactions of imidazopyridines (1a) with substrates 2a catalyzed by electron were performed normally according to the general procedure described above, although at intermediate conversion light irradiation was ceased. Conversion of $\mathbf{1 a}$ was determined by means of ${ }^{1} \mathrm{H}$ NMR analysis from an aliquot from the reaction mixture (toluene as internal standard, $b p=110{ }^{\circ} \mathrm{C}$ ). The reaction mixture was subsequently degassed and then stirred in the dark for 2 hours. After that time, in all cases the reactions did not evolve further (Figure S5). These results demonstrate that light is a necessary component of the reaction. However, they do not definitively rule out a radical chain mechanism, the data show that this propagation process must be short-live.

\section{References}

${ }^{1}$ T. Nihei; N. Iwai; T. Matsuda and T. Kitazume, J. Org. Chem., 2005, 70, 5912.

2 (a) L. Dymińska, Bioorg. Med. Chem. 2015, 23, 6087; (b) M. S. Christodoulou; F. Colombo; D. Passarella; G. Ieronimo; V. Zuco; M. D. Cesare; F. Zunino, Bioorg. Med. Chem. 2011, 19. 1649; (c) C. Mukherjee; K. T. Watanabe; E. R. Biehl, Tetrahedron Lett. 2012, 53, 6008.

${ }^{3}$ (a) M. P. D. Rafael; C. B. B. Antonio, Org. Lett. 2016, 18, 3034; (b) C. B. B. Antonio, Chem. Eur. J. 2017, 23, 16980 
Copies ${ }^{1} \mathrm{H}$ NMR, ${ }^{13} \mathrm{C}$ NMR, ${ }^{19} \mathrm{~F}$ NMR

2-bromo-2,2-difluoro-1-( $p$-tolyl)ethan-1-one (2b)

${ }^{1} \mathrm{H}$ NMR (400 MHz, $\left.\mathrm{CDCl}_{3}\right)$ :

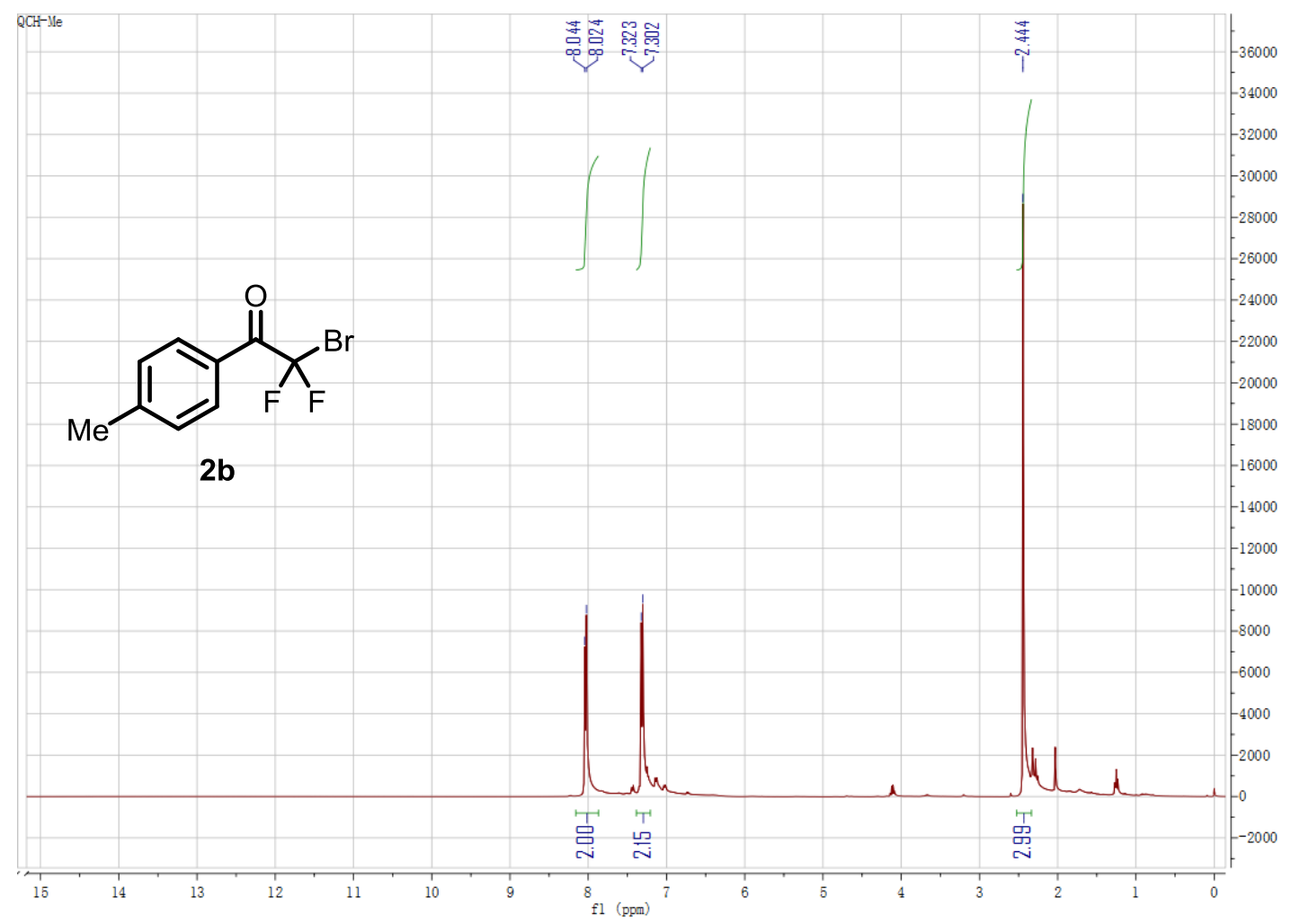

${ }^{19}$ F NMR (376 MHz, CDCl3):

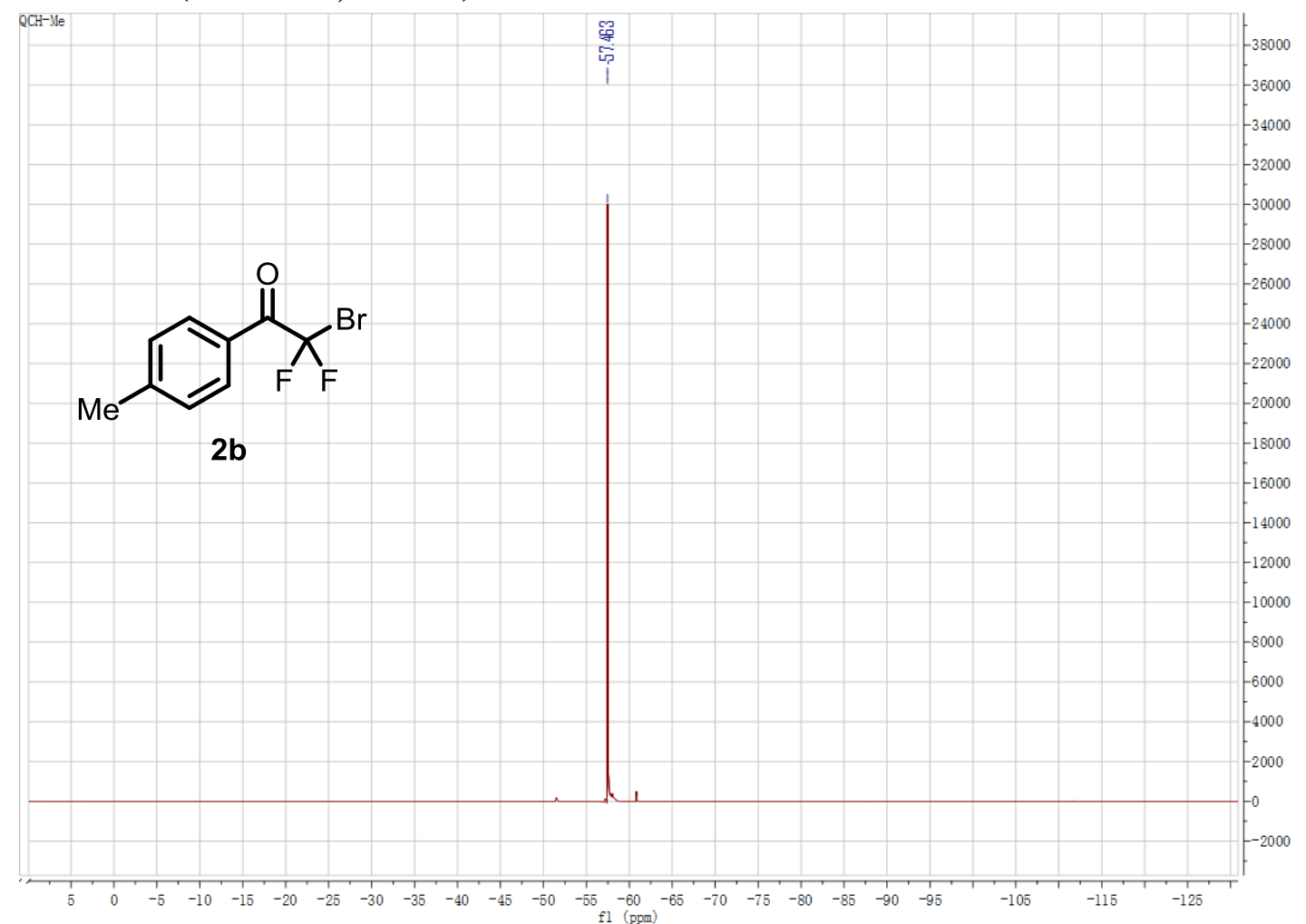


${ }^{13} \mathrm{C}$ NMR (100 MHz, $\left.\mathrm{CDCl}_{3}\right)$ :

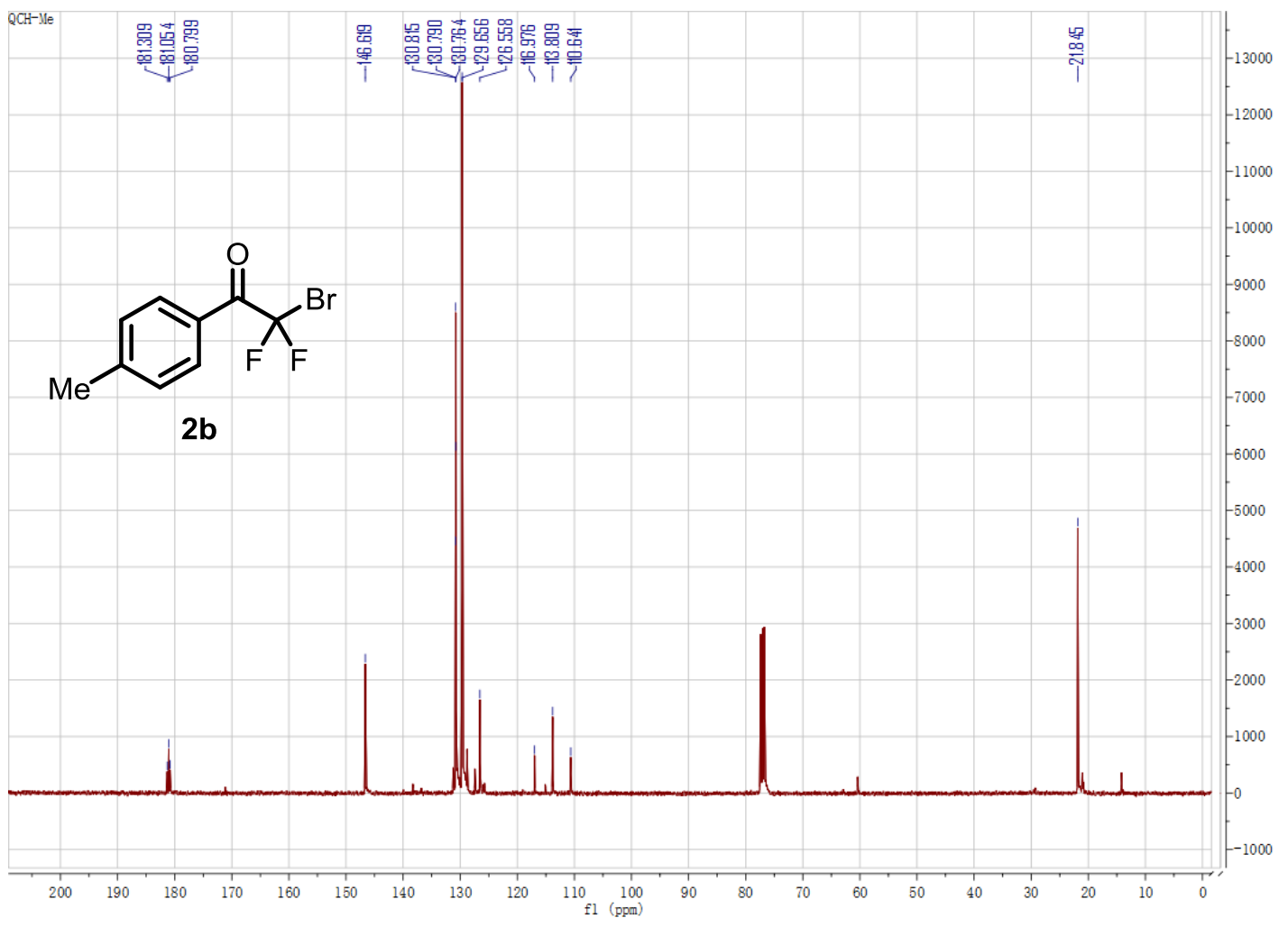


2-bromo-2,2-difluoro-1-(4-methoxyphenyl)ethan-1-one (2c)

${ }^{1}$ H NMR (400 MHz, CDCl3):

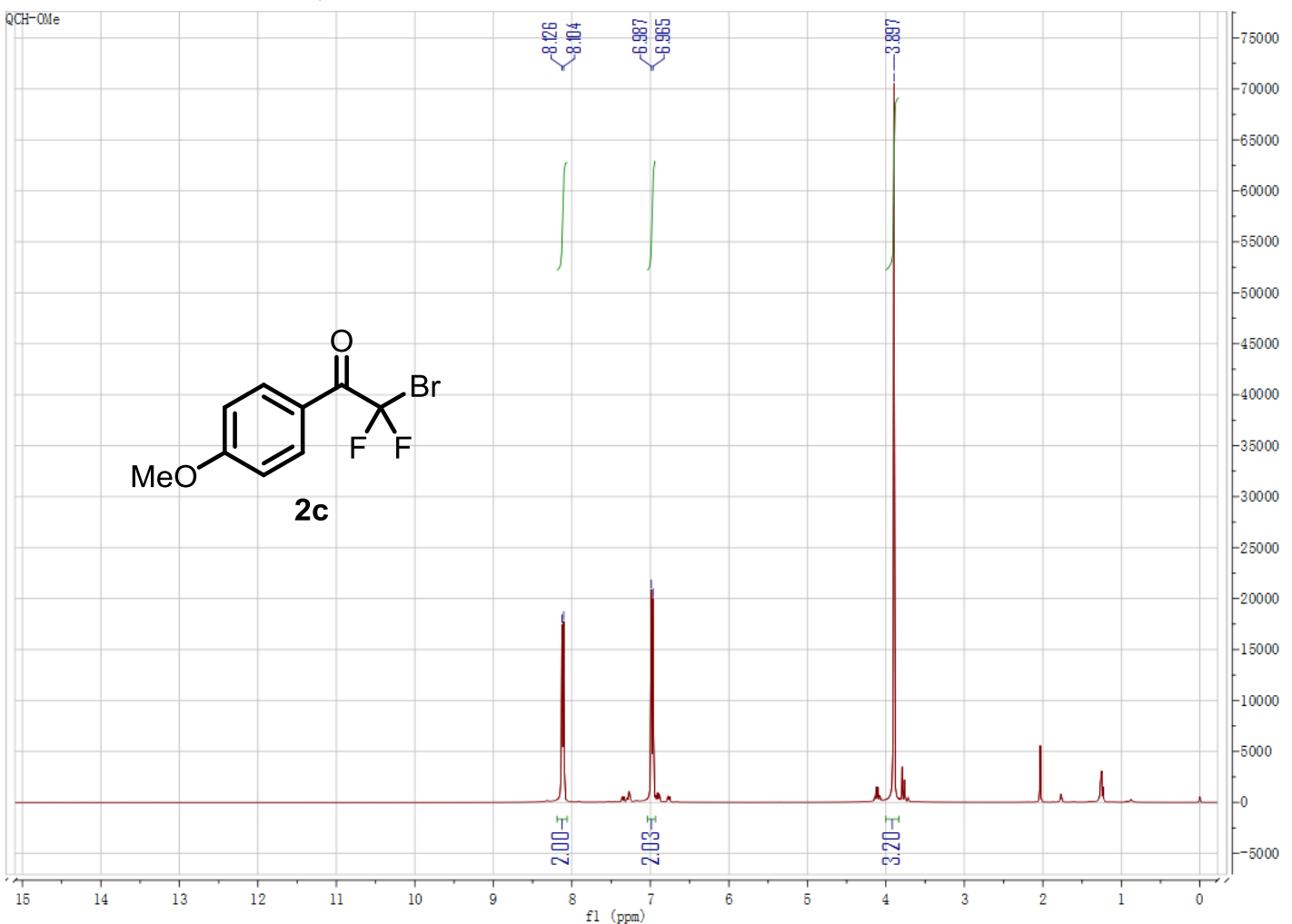

${ }^{19}$ F NMR (376 MHz, CDCl3):

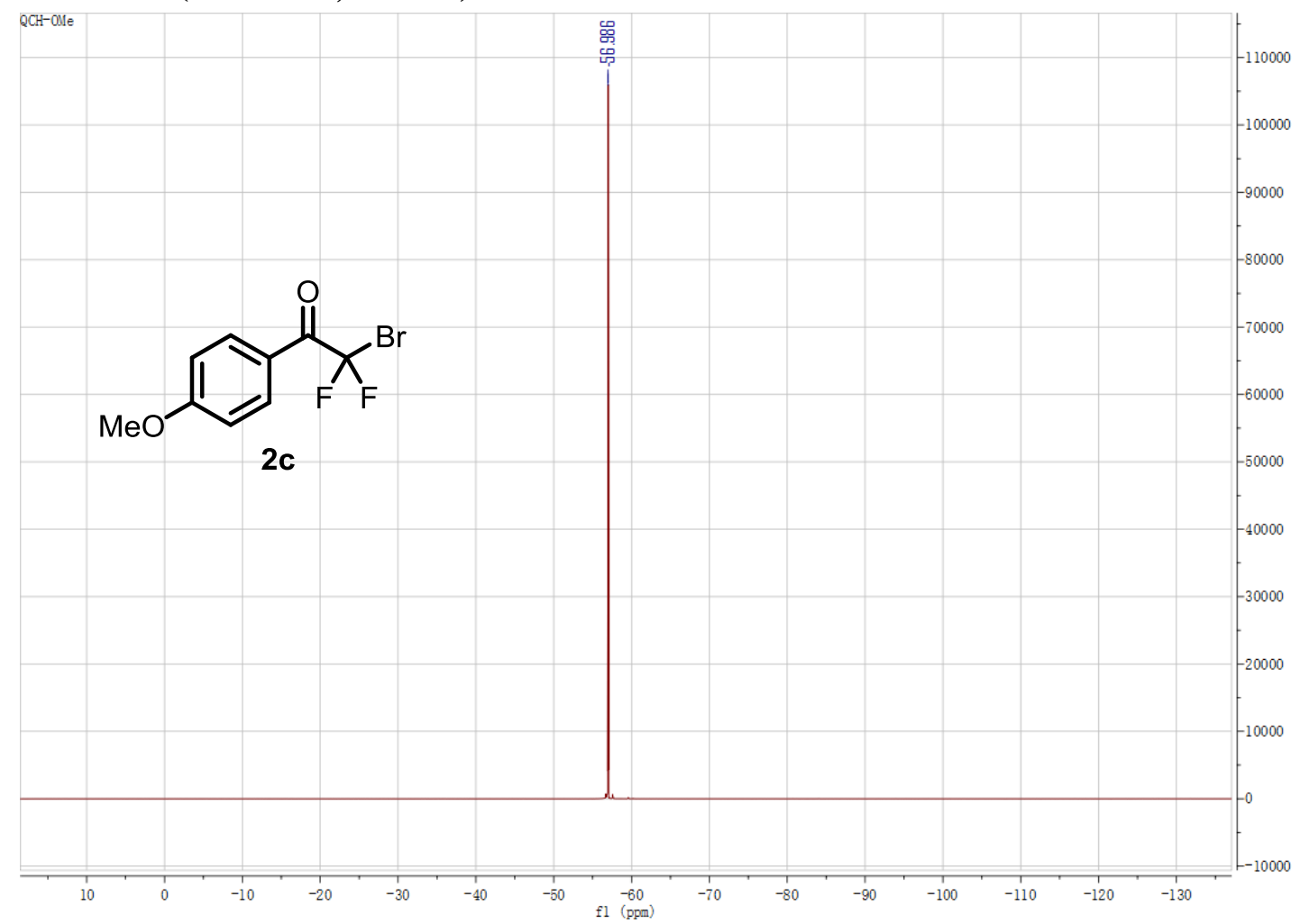


${ }^{13} \mathrm{C}$ NMR (100 MHz, CDCl3):

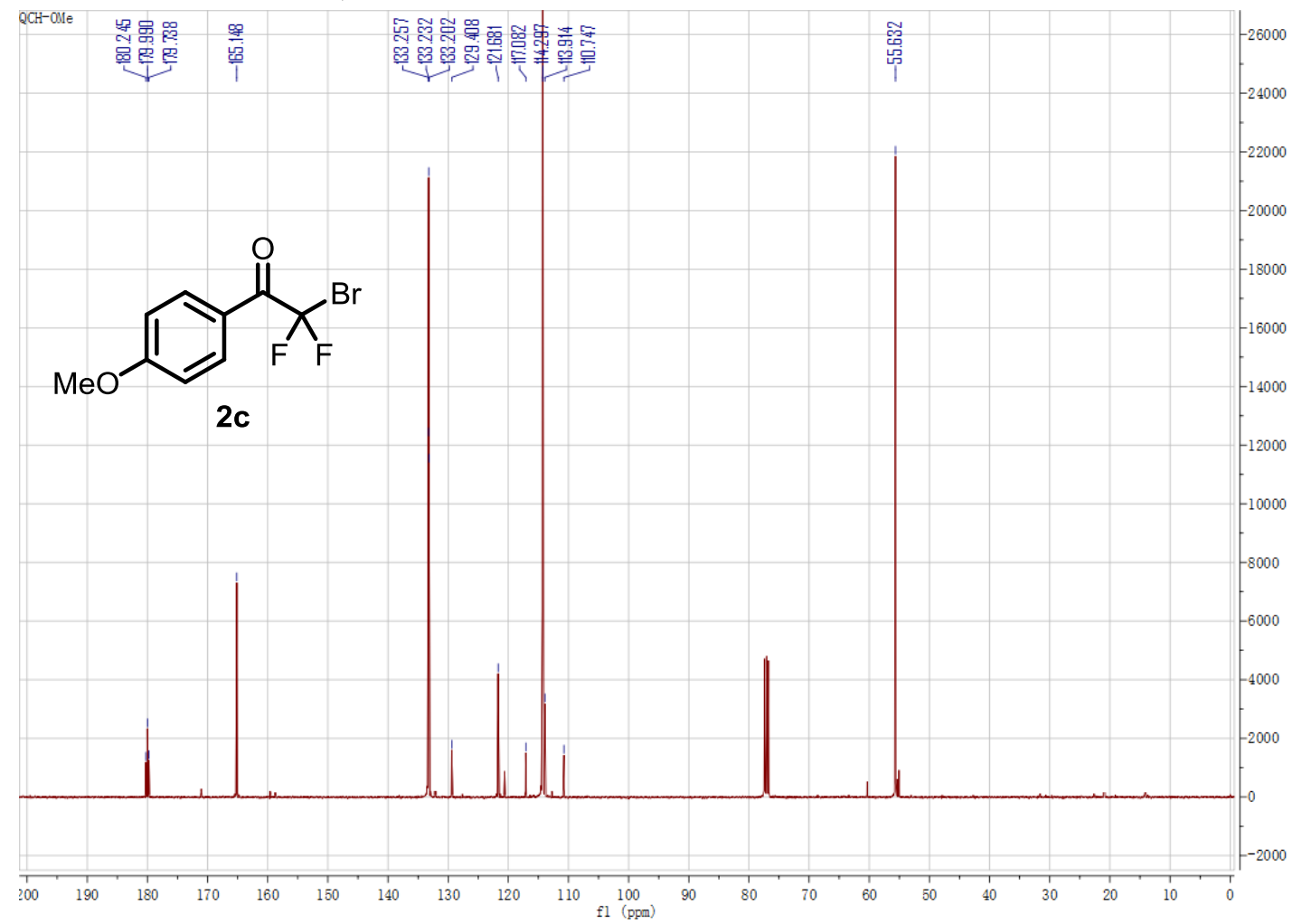


2-bromo-1-(4-(tert-butyl)phenyl)-2,2-difluoroethan-1-one (2d) ${ }^{1} \mathrm{H}$ NMR (400 MHz, $\left.\mathrm{CDCl}_{3}\right)$ :

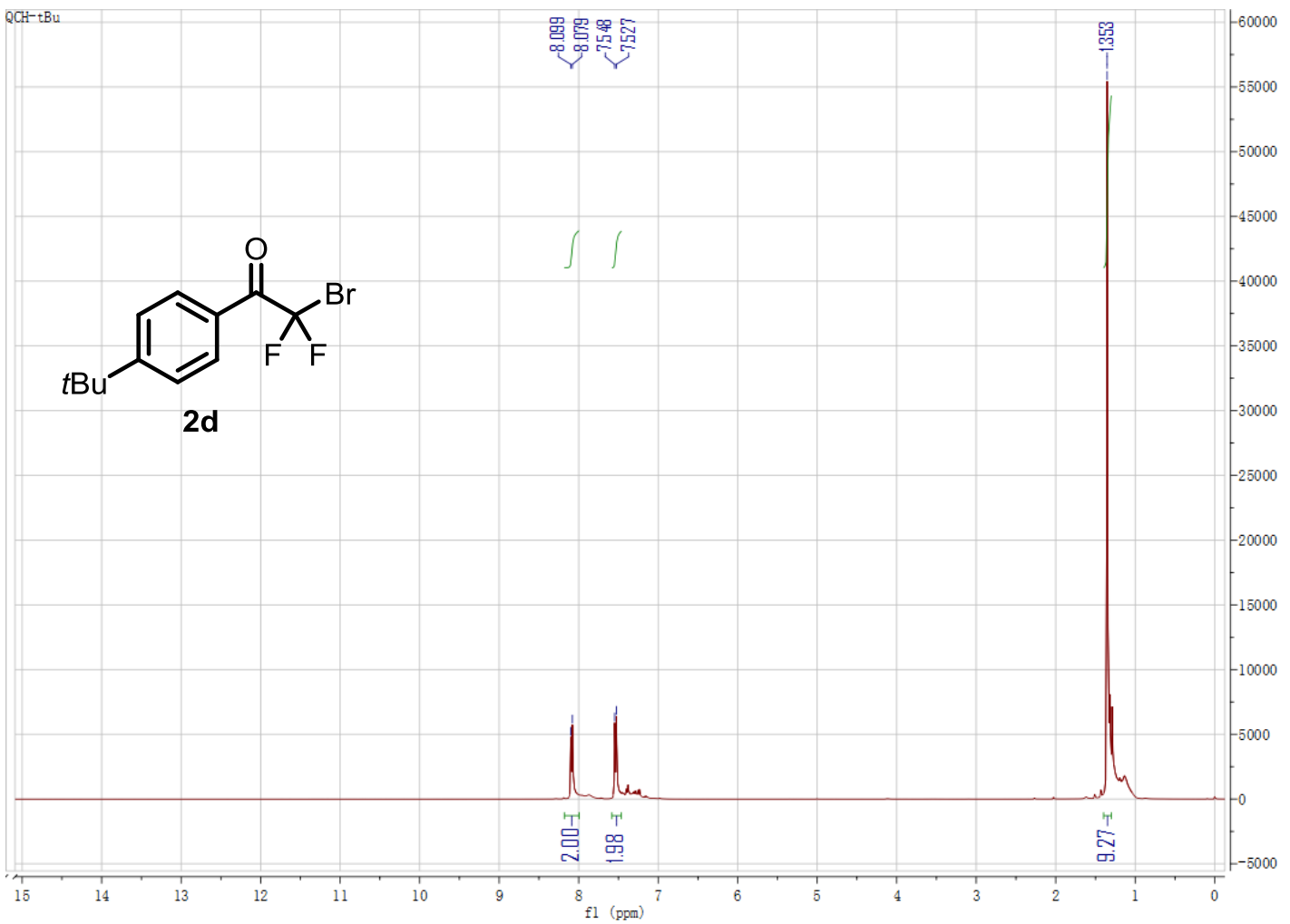

${ }^{19}$ F NMR (376 MHz, CDCl 3$)$ :

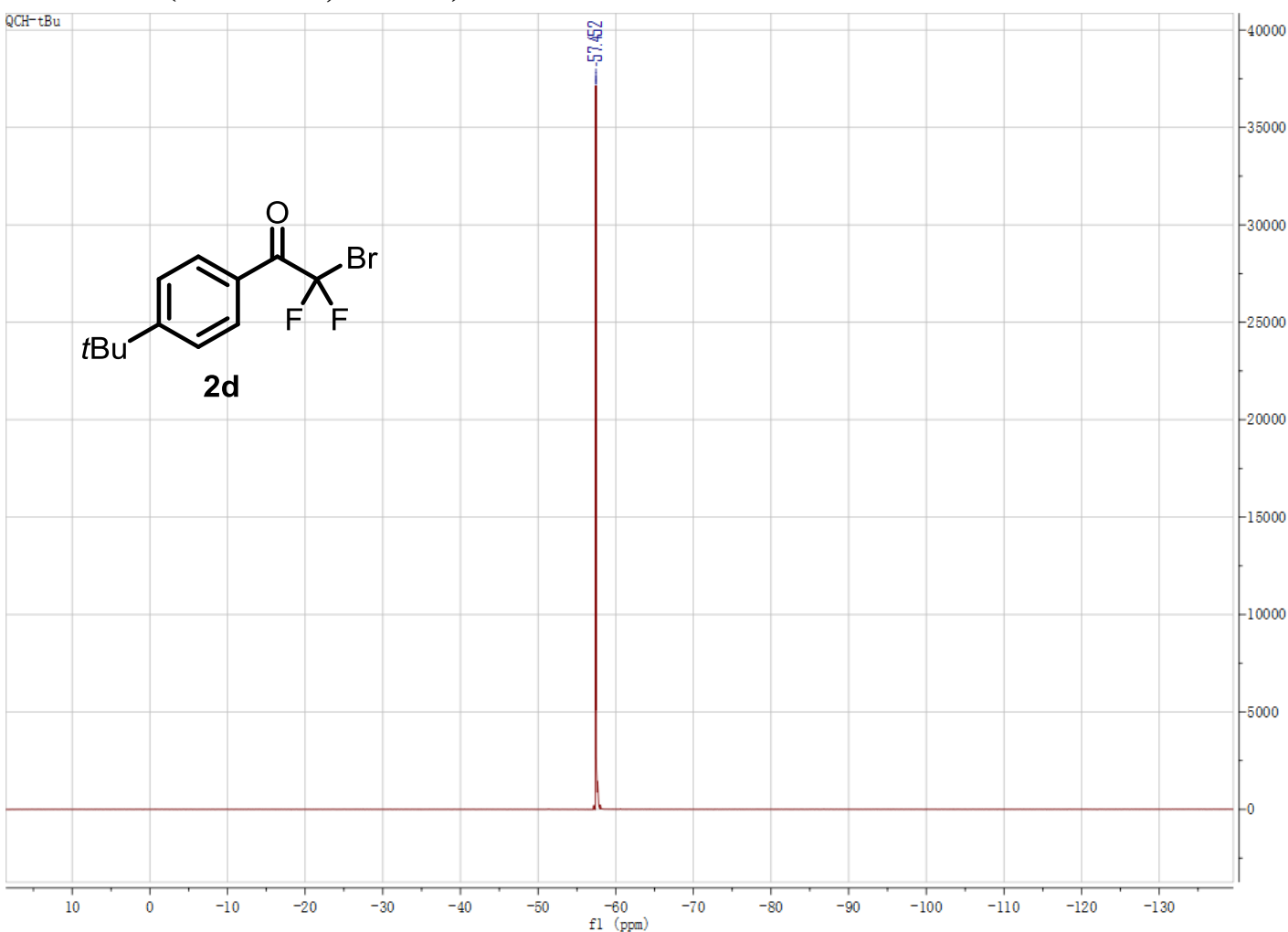


${ }^{13} \mathrm{C}$ NMR (100 MHz, CDCl3):

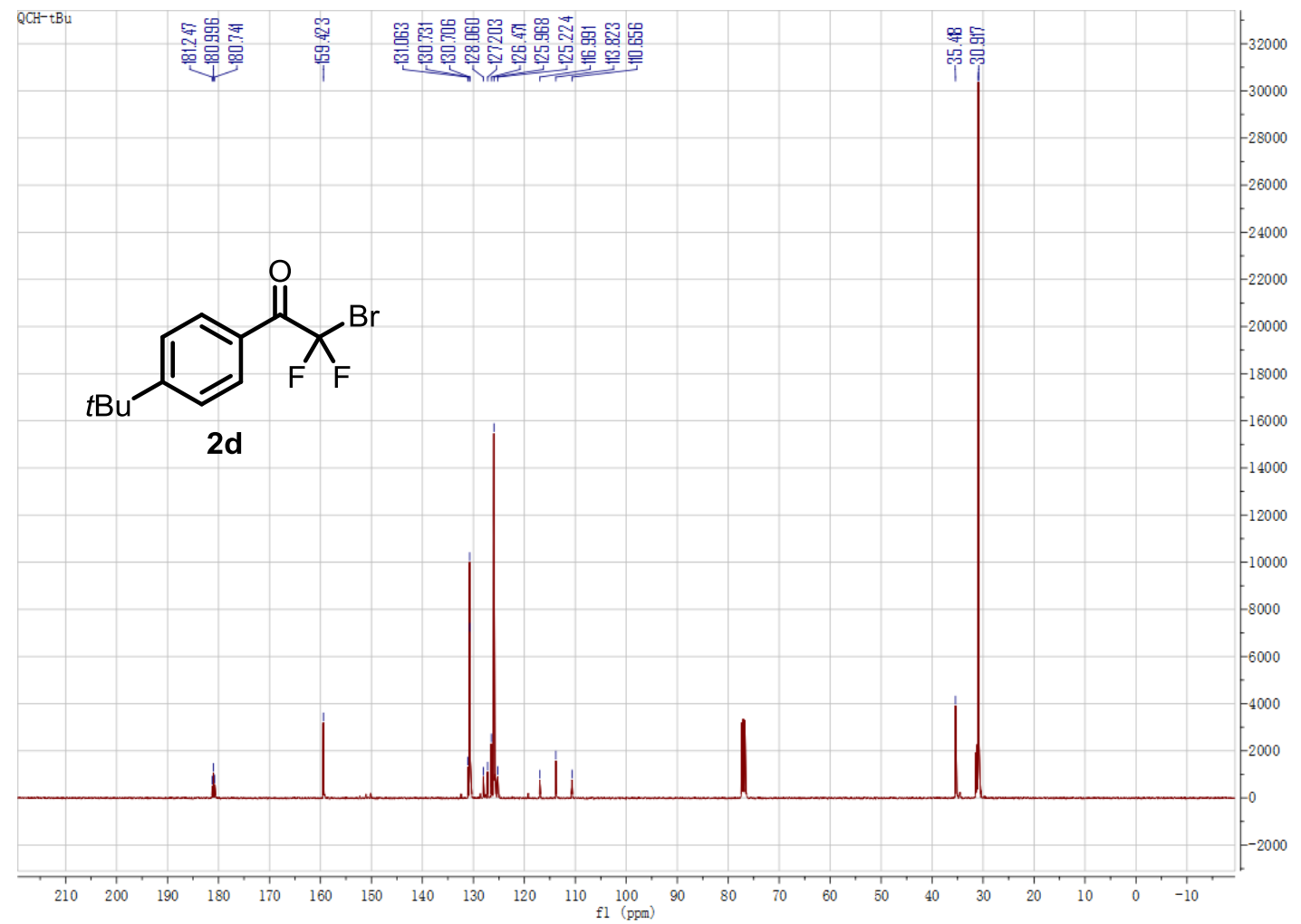


2-bromo-2,2-difluoro-1-(4-fluorophenyl)ethan-1-one (2e)

${ }^{1} \mathrm{H}$ NMR (400 MHz, $\left.\mathrm{CDCl}_{3}\right)$ :

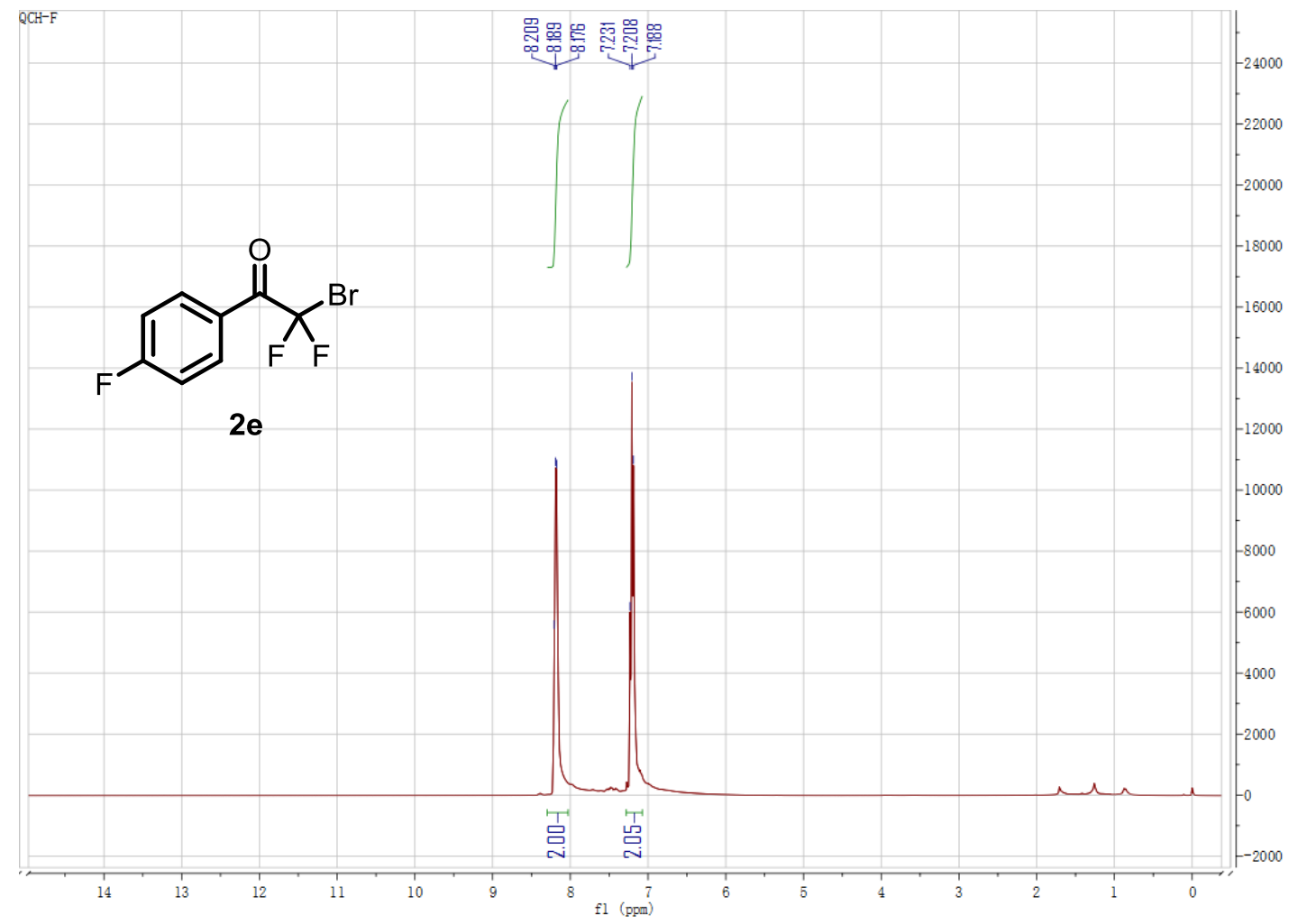

${ }^{19}$ F NMR (376 MHz, CDCl3): 


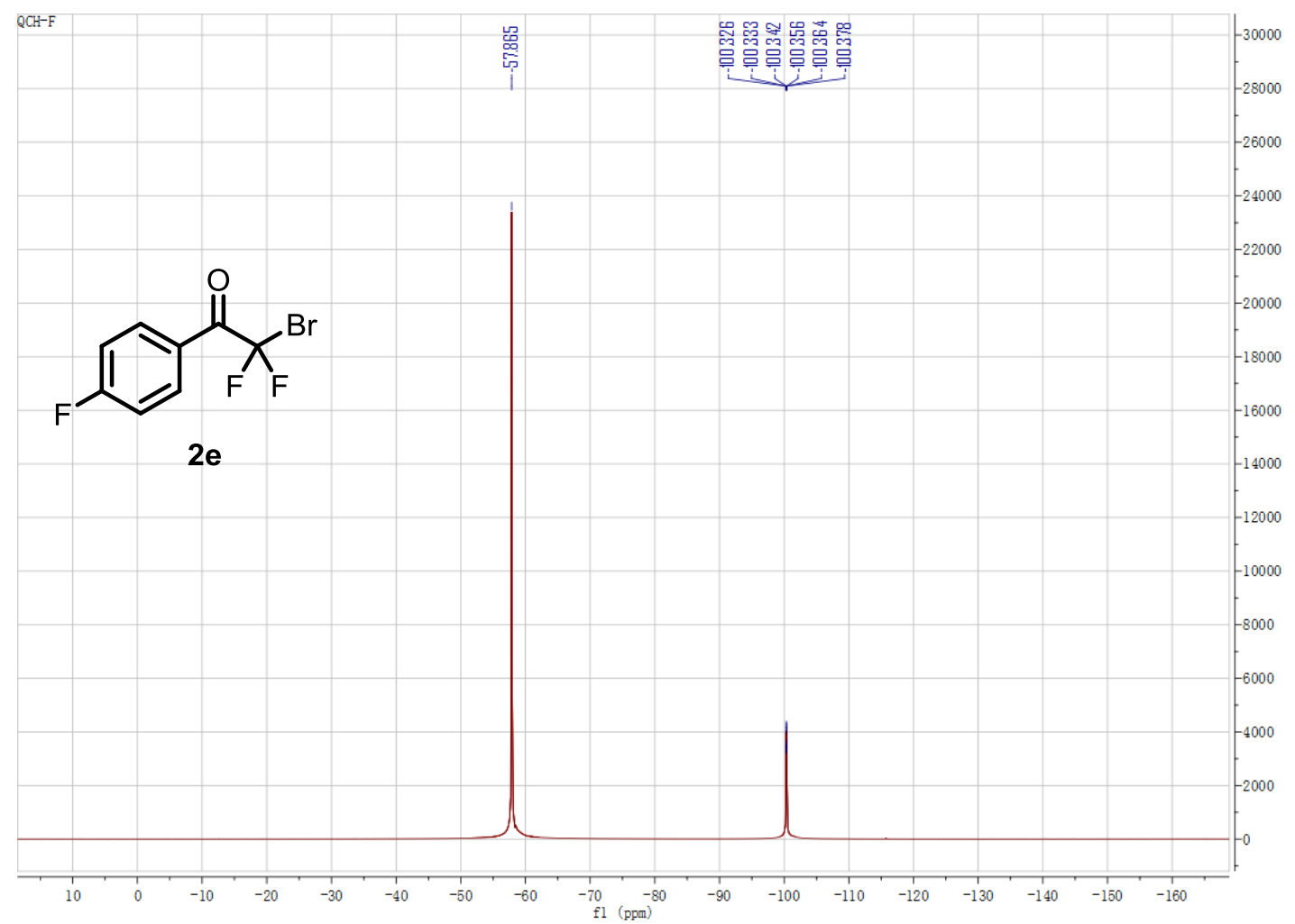

${ }^{13} \mathrm{C}$ NMR (100 MHz, CDCl3):

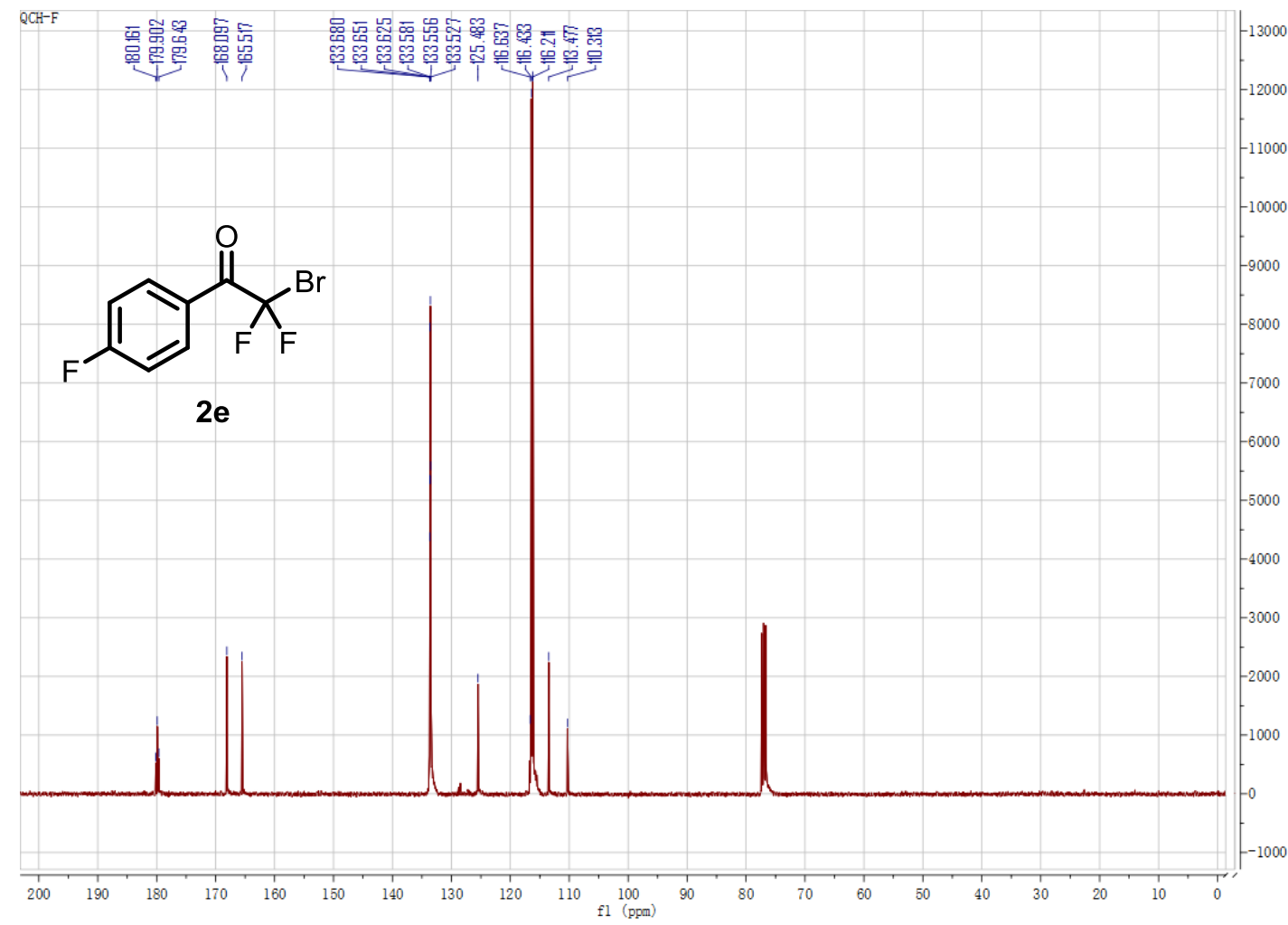


2-bromo-2,2-difluoro-1-(naphthalen-2-yl)ethan-1-one (2f) ${ }^{1} \mathrm{H}$ NMR (400 MHz, CDCl3):

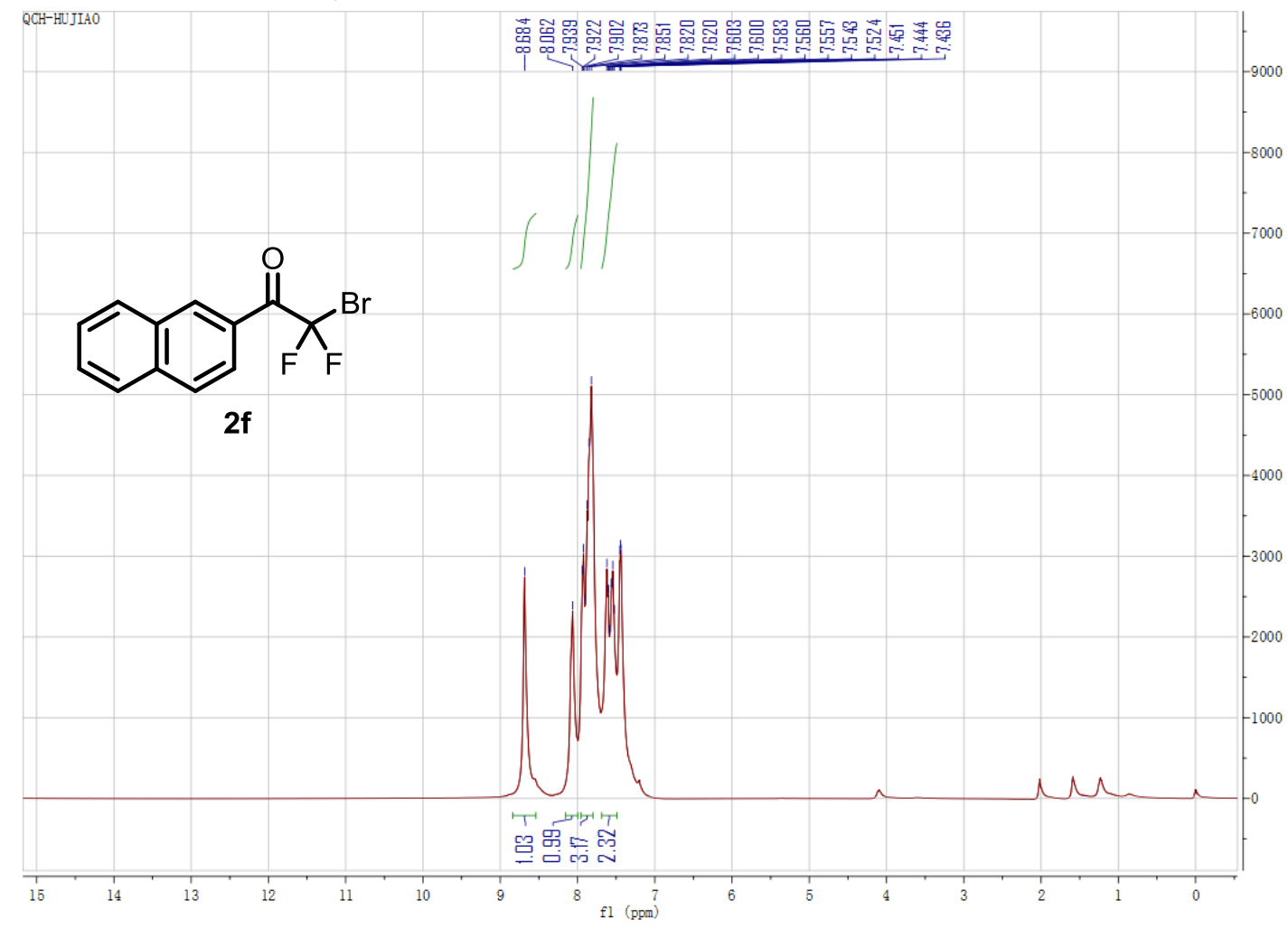


${ }^{19}$ F NMR (376 MHz, CDCl3):

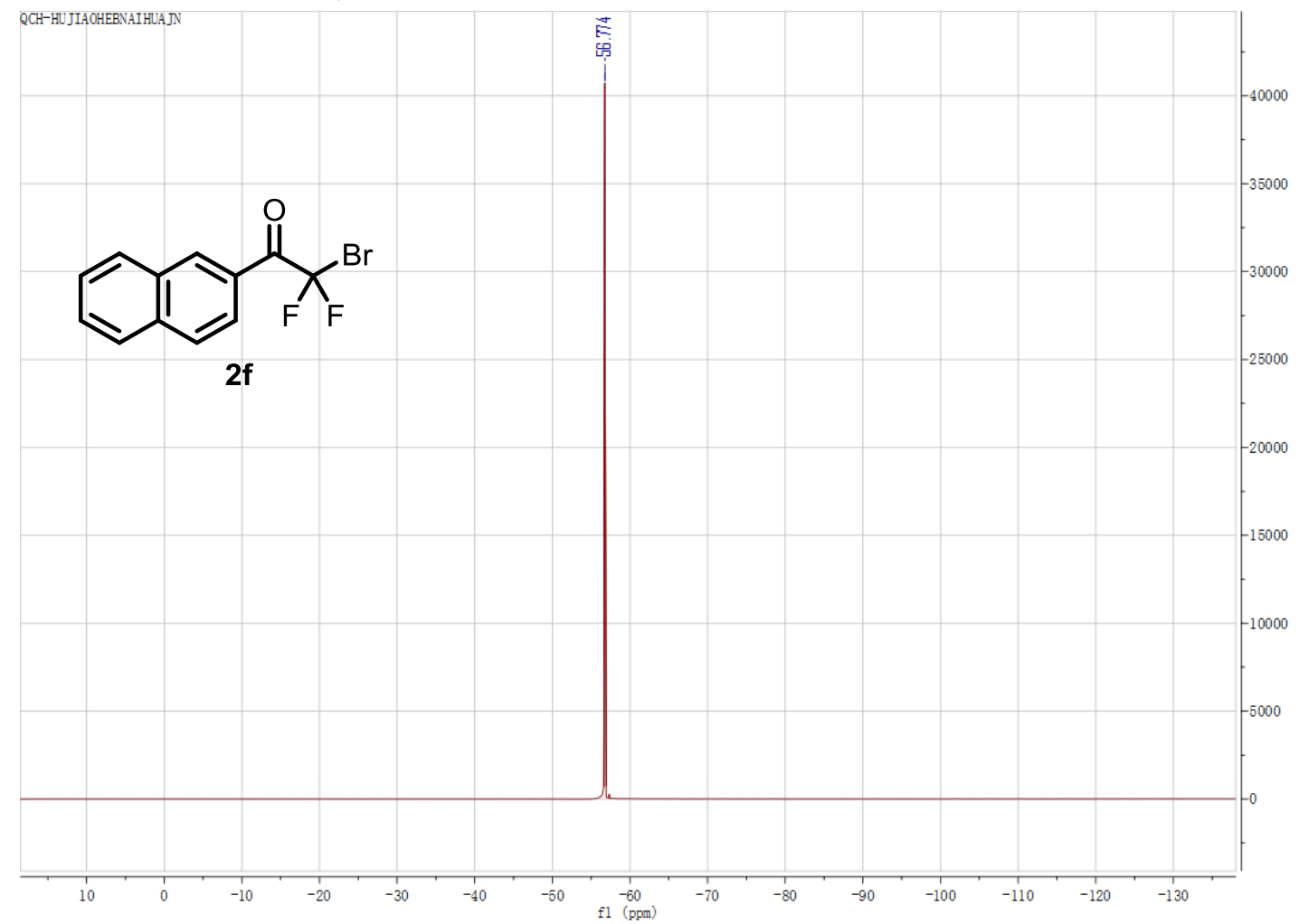

${ }^{13} \mathrm{C}$ NMR (100 MHz, CDCl3):

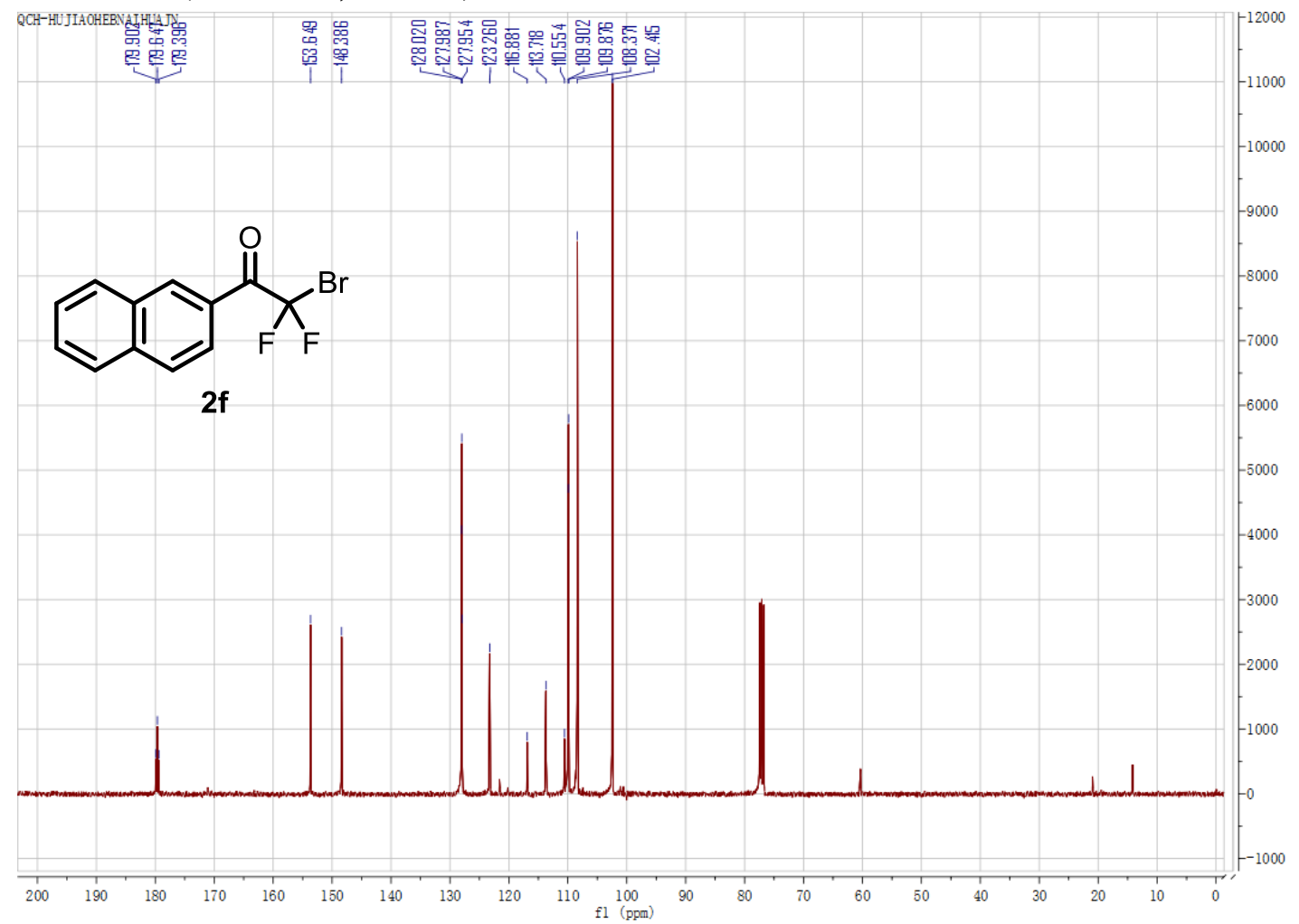


2-bromo-2,2-difluoro-1-(thiophen-2-yl)ethan-1-one (2g)

${ }^{1}$ H NMR (400 MHz, CDCl3):

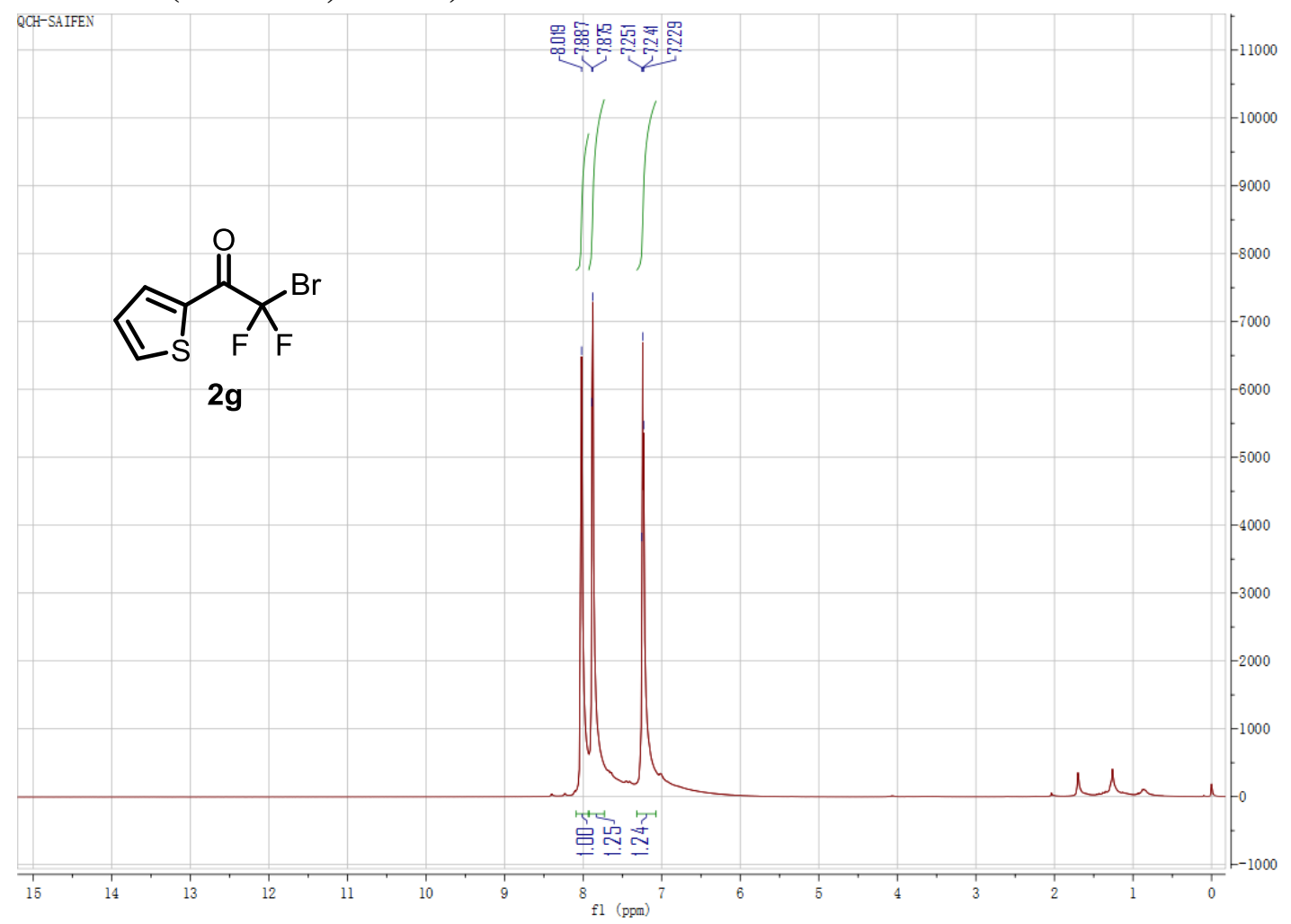


${ }^{19}$ F NMR (376 MHz, CDCl3):

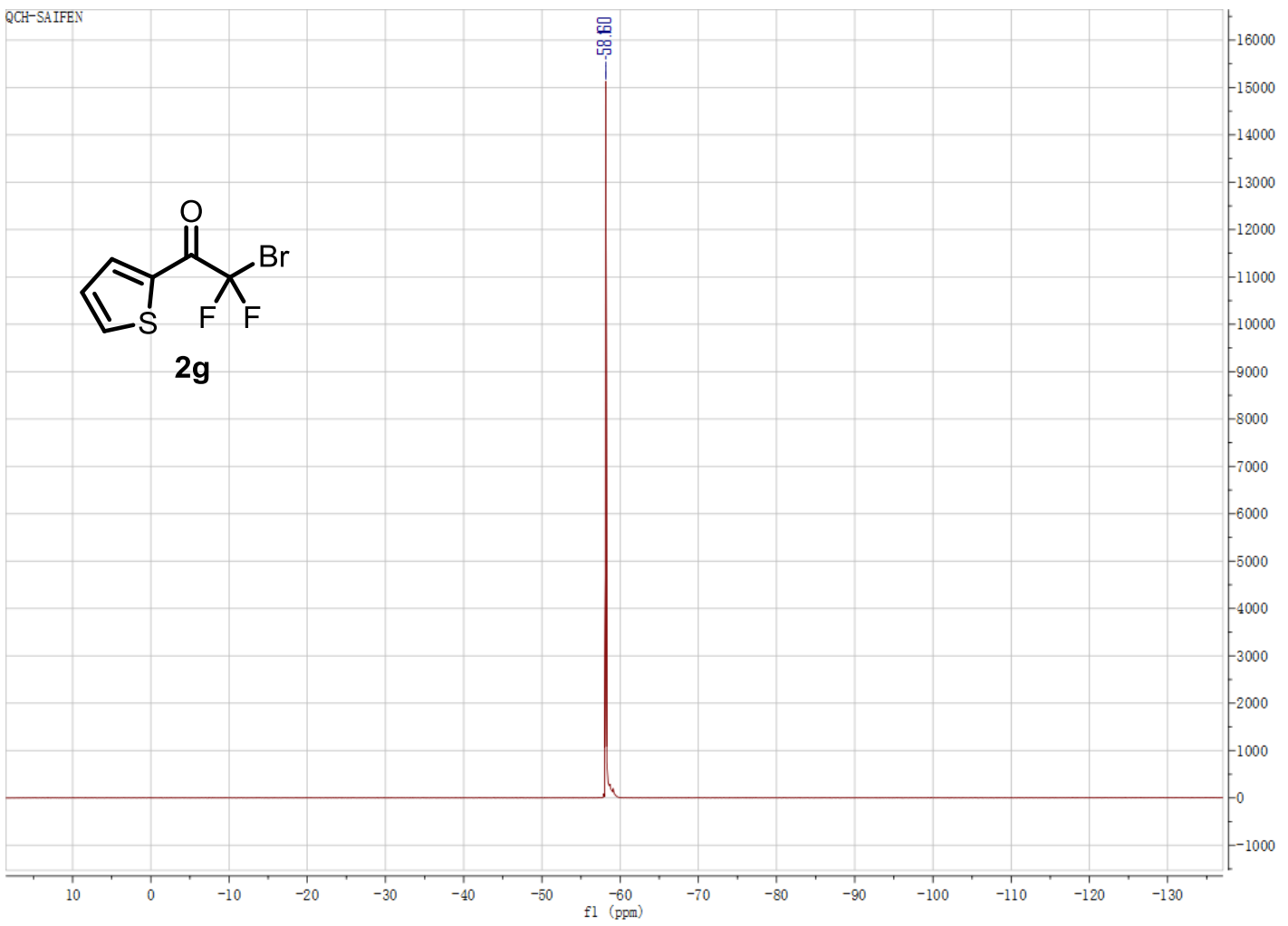

${ }^{13} \mathrm{C}$ NMR (100 MHz, CDCl 3$)$ :

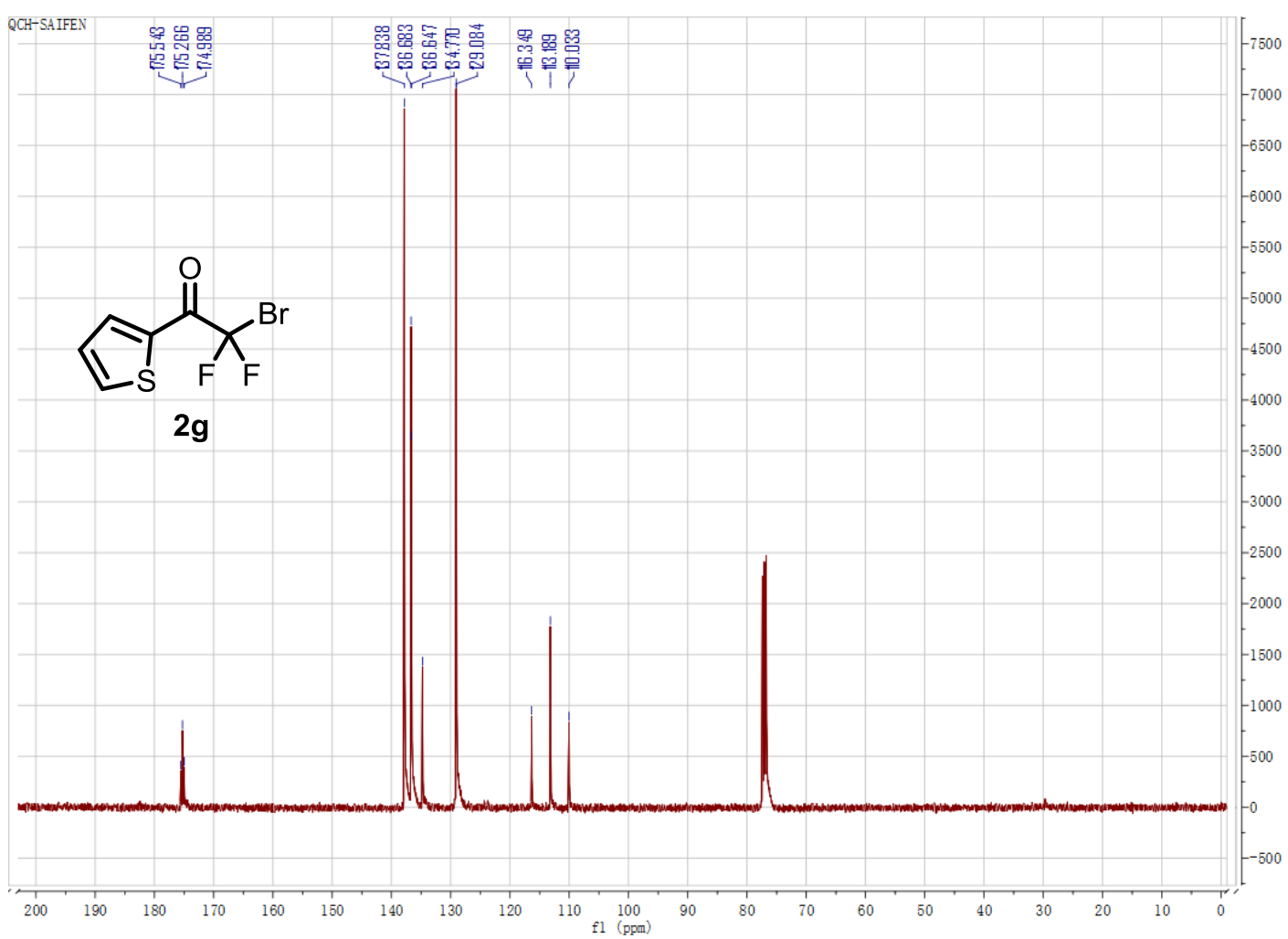


1-(benzo[d][1,3]dioxol-5-yl)-2-bromo-2,2-difluoroethan-1-one (2h) ${ }^{1} \mathrm{H}$ NMR (400 MHz, $\left.\mathrm{CDCl}_{3}\right)$ : 


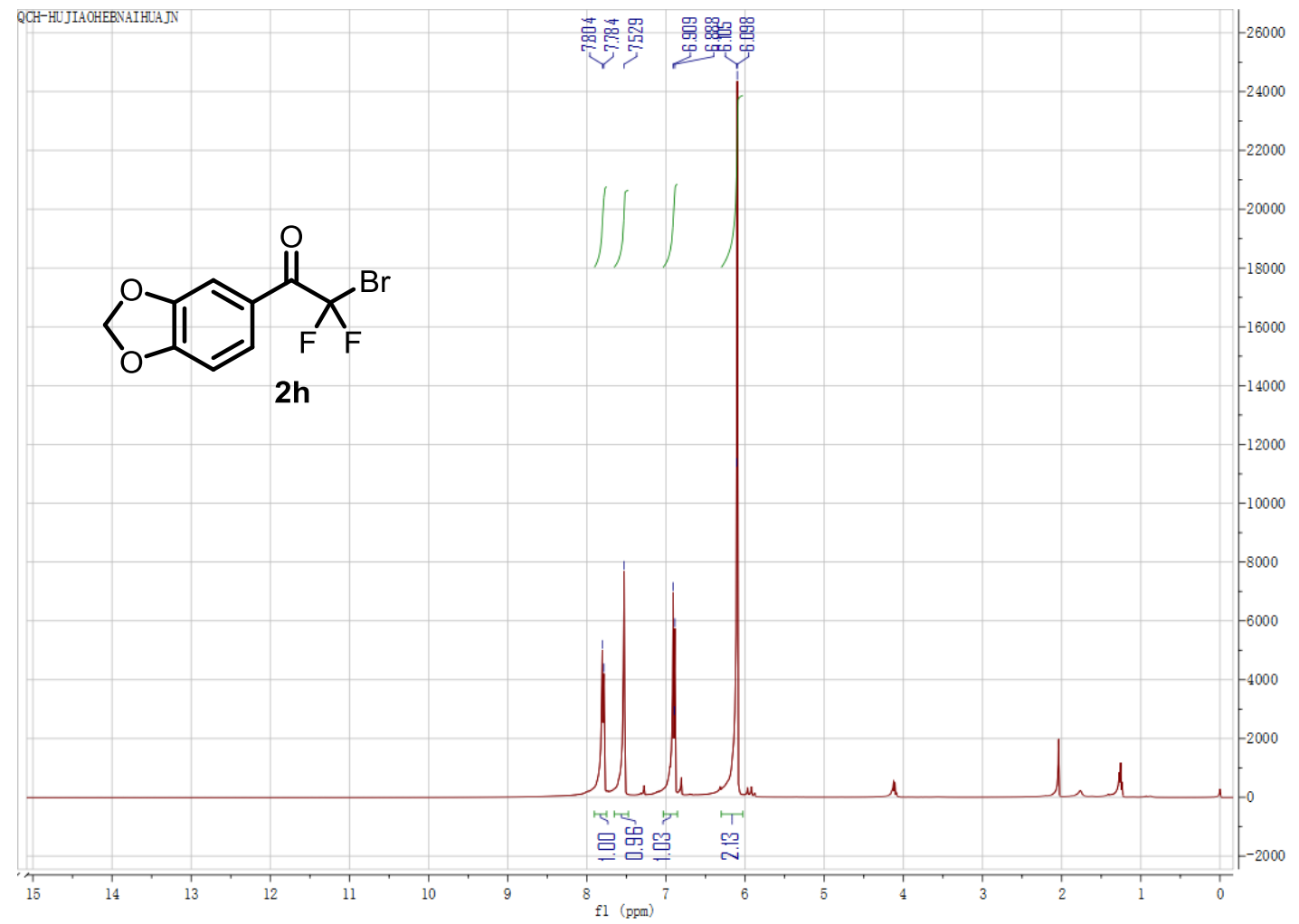

${ }^{19}$ F NMR (376 MHz, CDCl 3 ):

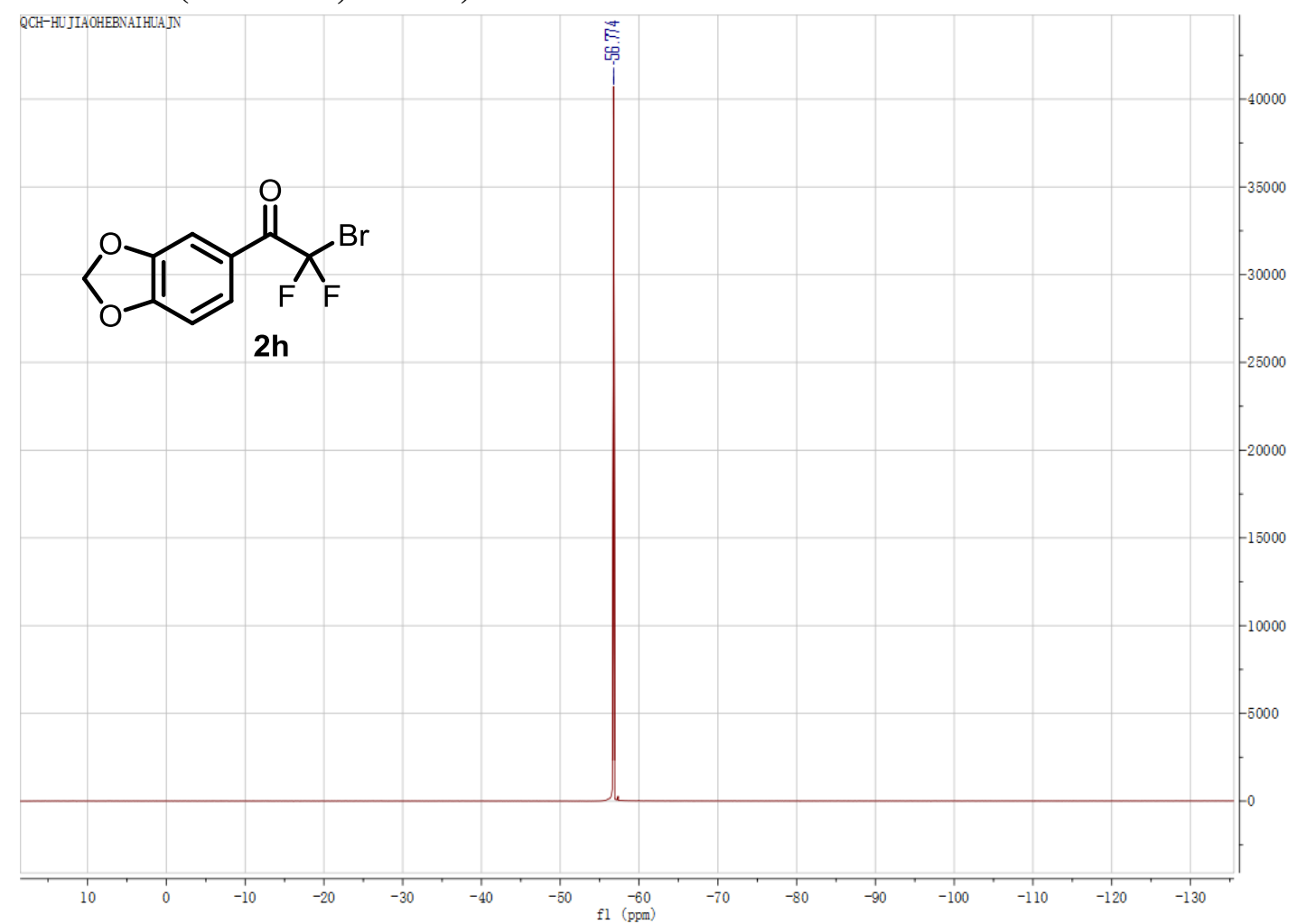

${ }^{13} \mathrm{C}$ NMR (100 MHz, $\left.\mathrm{CDCl}_{3}\right)$ : 


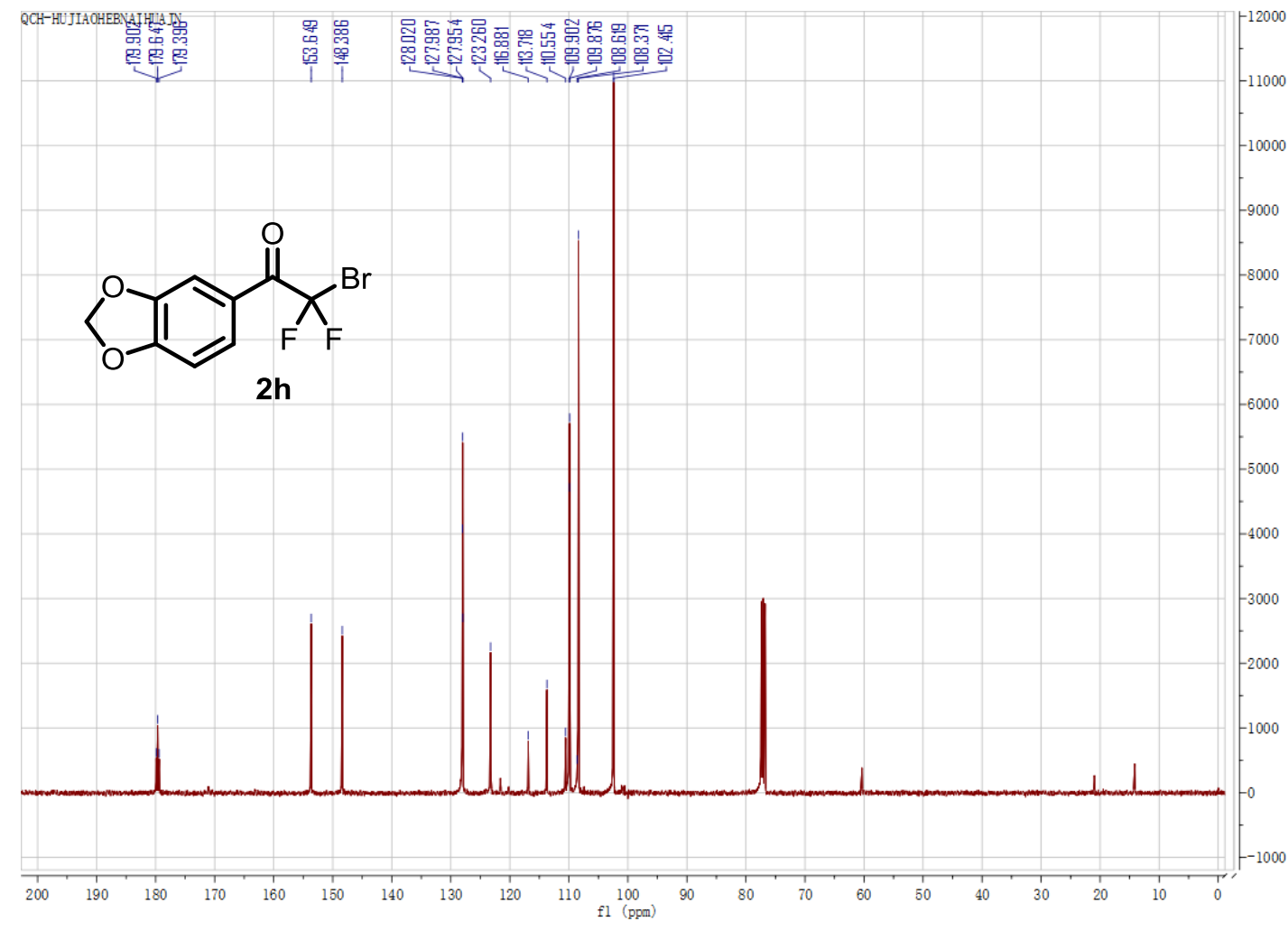


2,2-difluoro-2-(8-methyl-2-phenylimidazo[1,2-a]pyridin-3-yl)-1-phenylethan-1one (3aa)

${ }^{1} \mathrm{H}$ NMR (400 MHz, $\mathrm{CDCl}_{3}$ ):

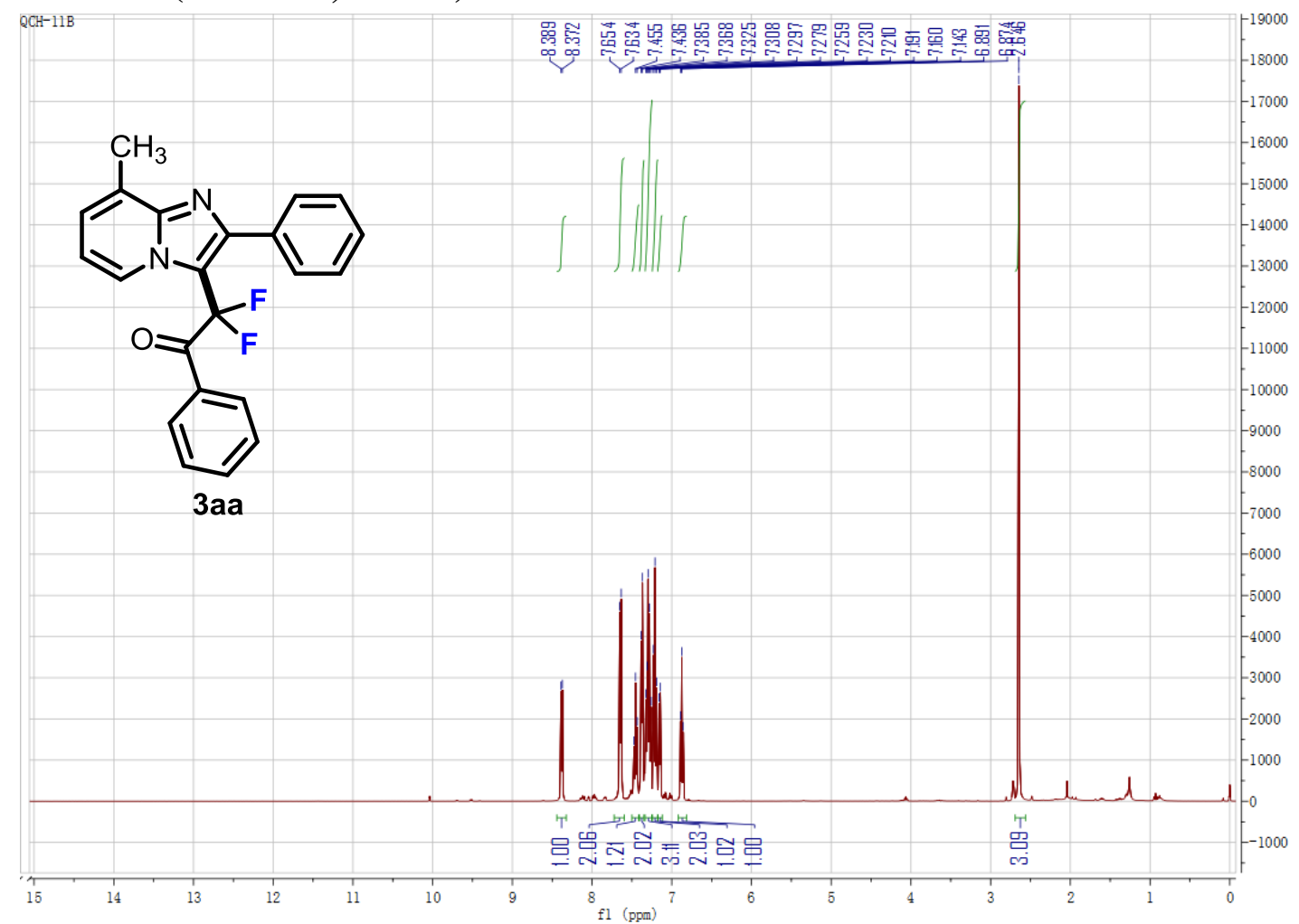

${ }^{19}$ F NMR (376 MHz, CDCl3):

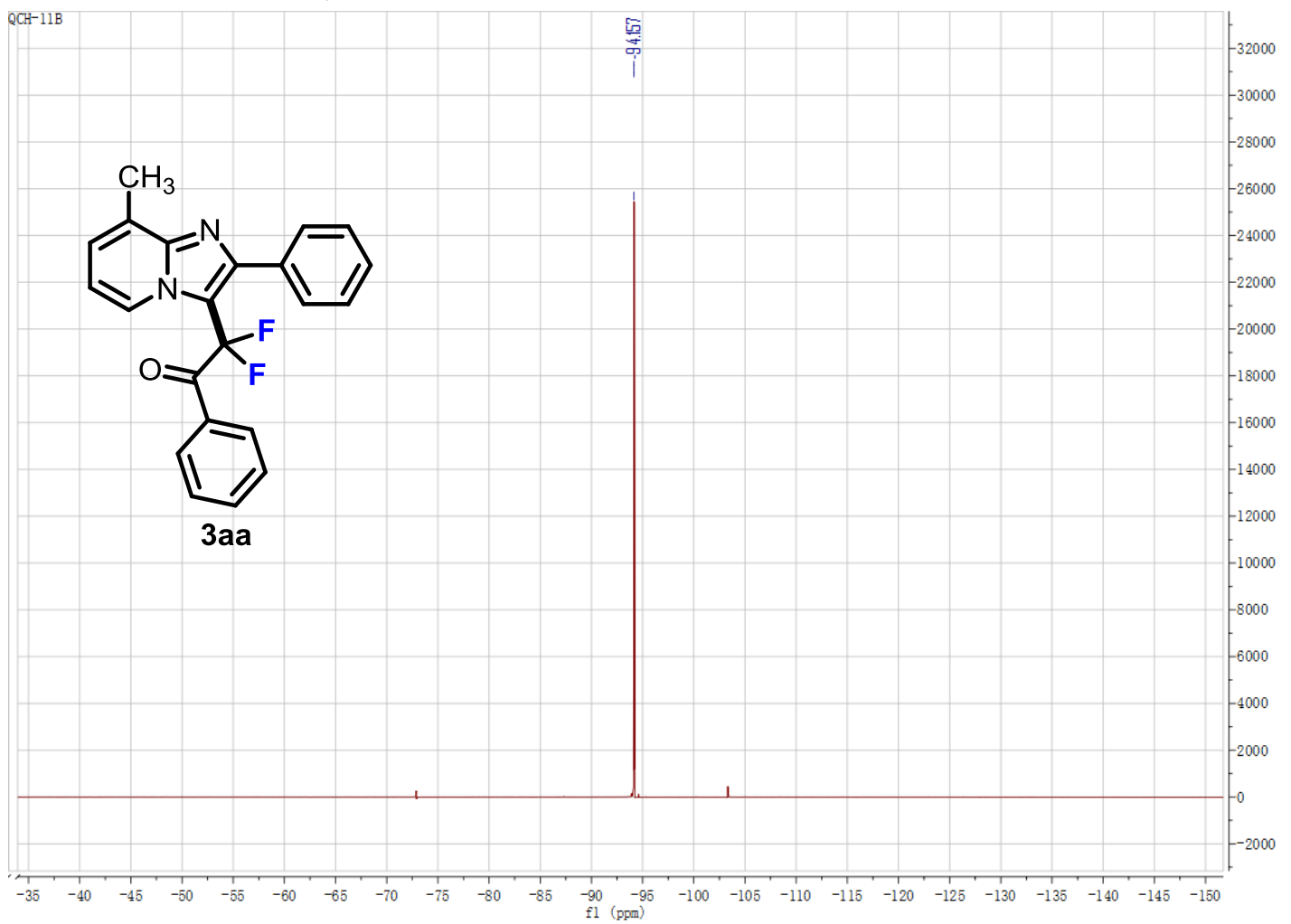


${ }^{13} \mathrm{C}$ NMR (100 MHz, CDCl3):

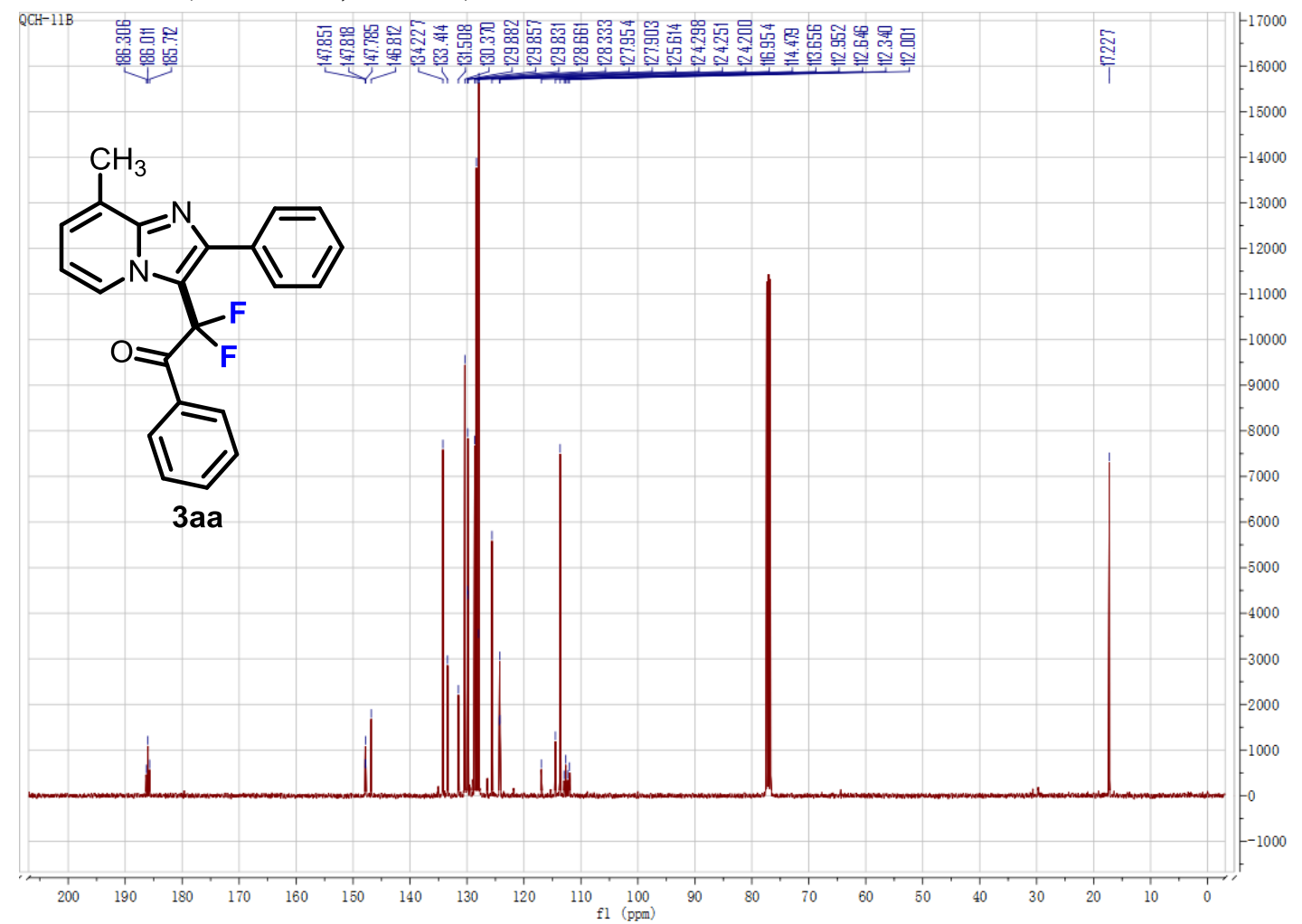


2,2-difluoro-2-(2-(4-methoxyphenyl)-8-methylimidazo[1,2-a]pyridin-3-yl)-1-phenylethan -1-one (3ab)

${ }^{1} \mathrm{H}$ NMR (400 MHz, $\left.\mathrm{CDCl}_{3}\right)$ :

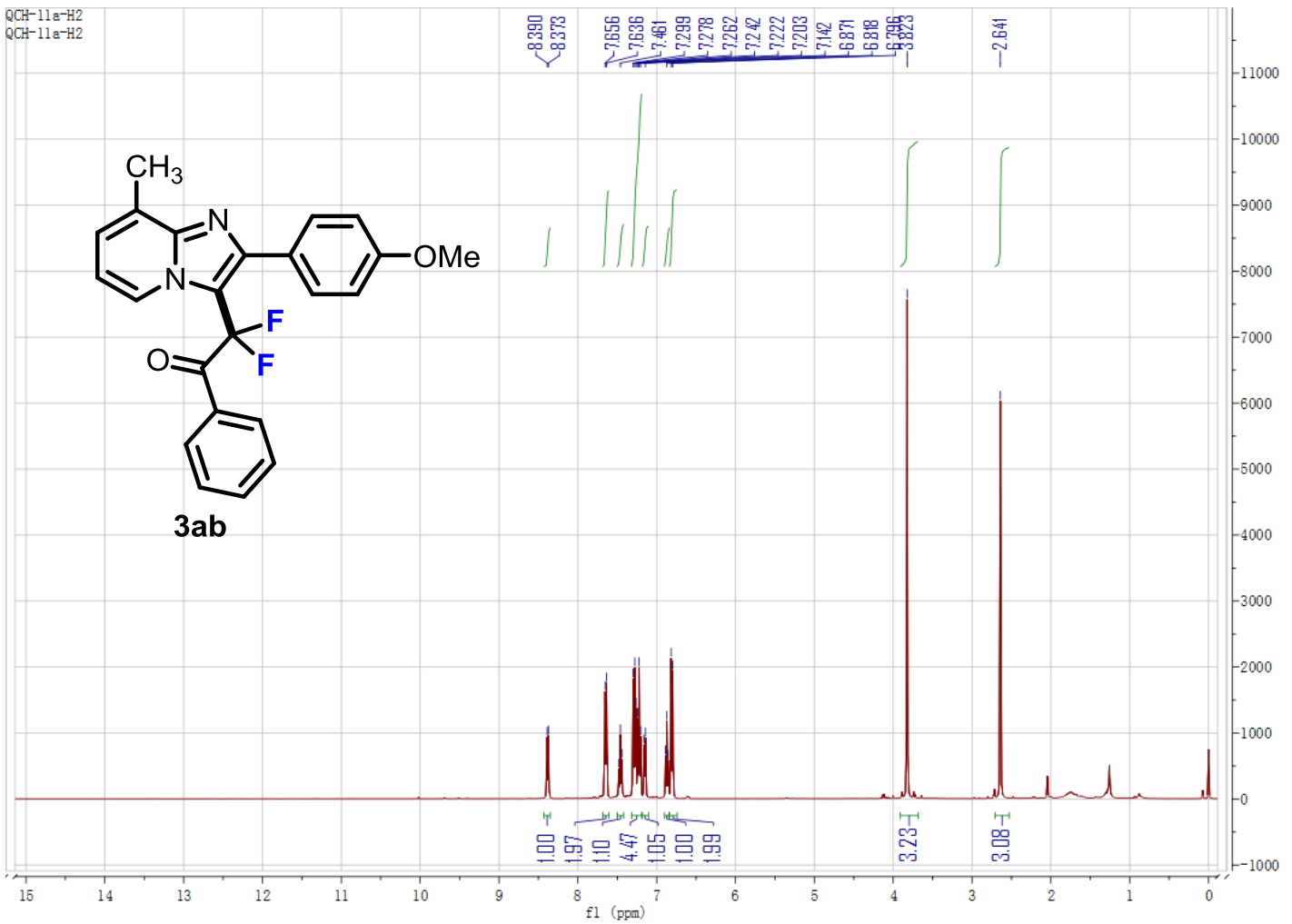

${ }^{19}$ F NMR (376 MHz, CDCl3): 


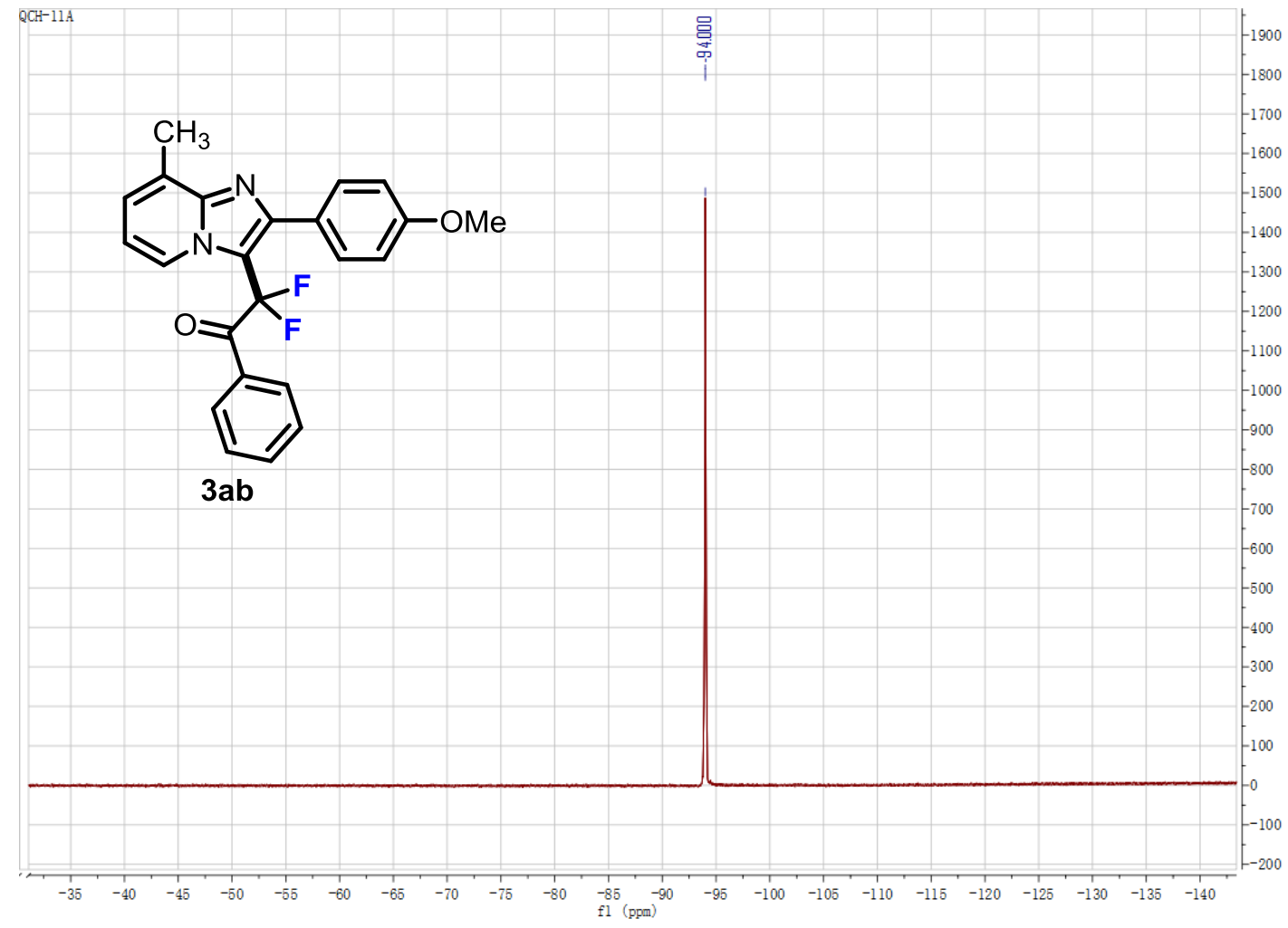

${ }^{13} \mathrm{C}$ NMR (100 MHz, $\left.\mathrm{CDCl}_{3}\right)$ :

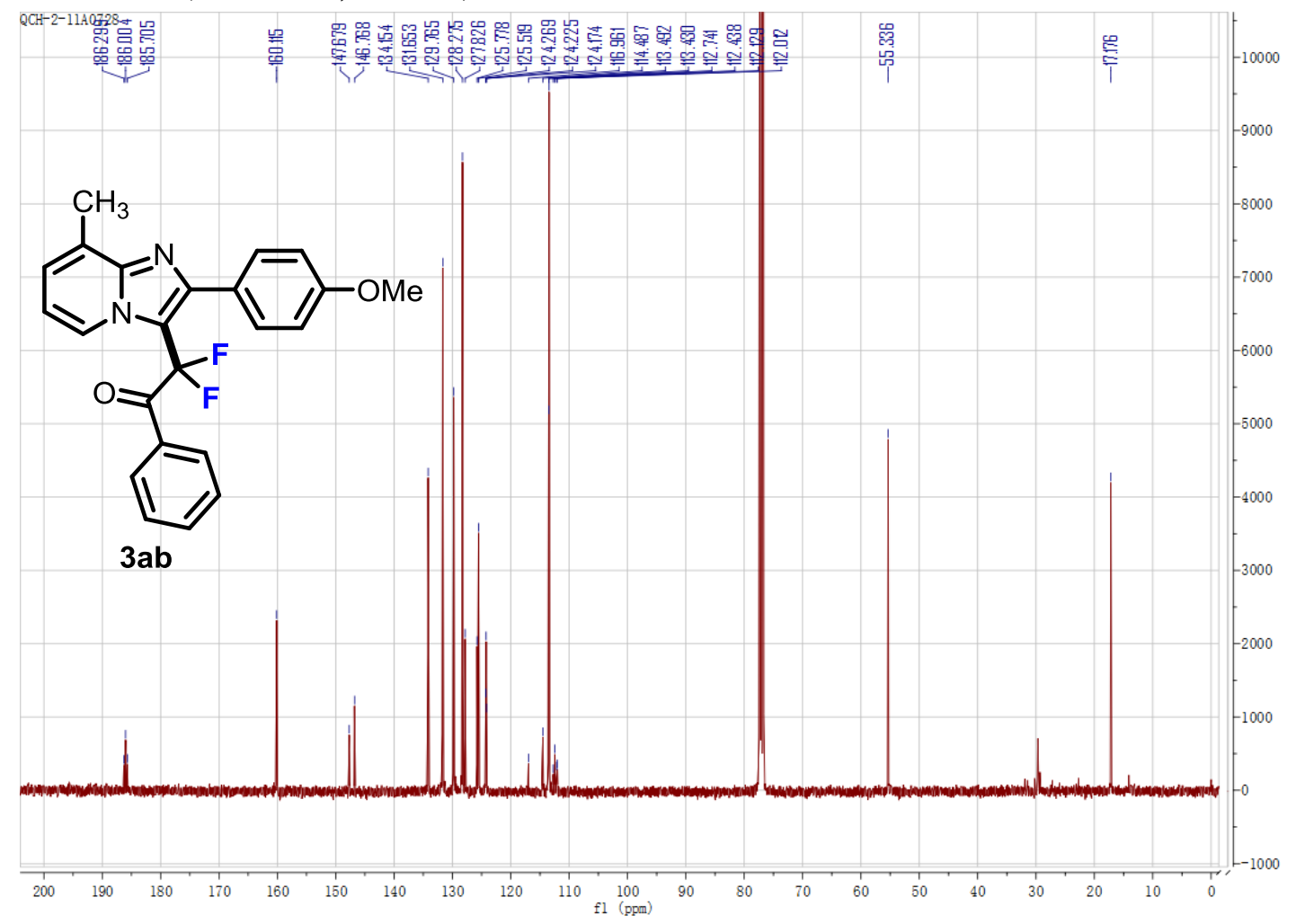


2,2-difluoro-2-(2-(2-fluorophenyl)-8-methylimidazo[1,2-a]pyridin-3-yl)-1-phenylethan-1 -one (3ac)

${ }^{1} \mathrm{H}$ NMR (400 MHz, $\left.\mathrm{CDCl}_{3}\right)$ :

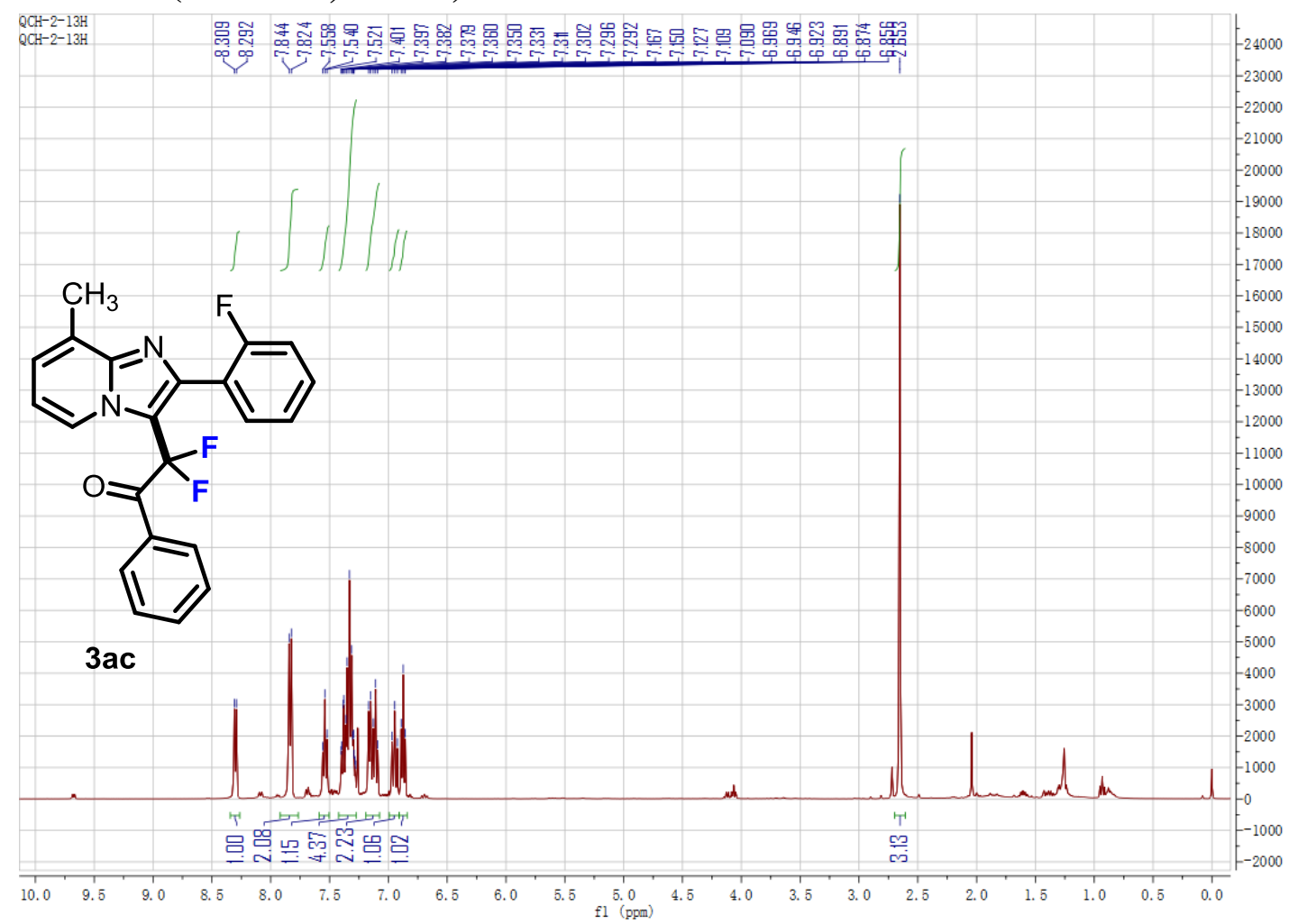

${ }^{19}$ F NMR (376 MHz, CDCl $)$ : 


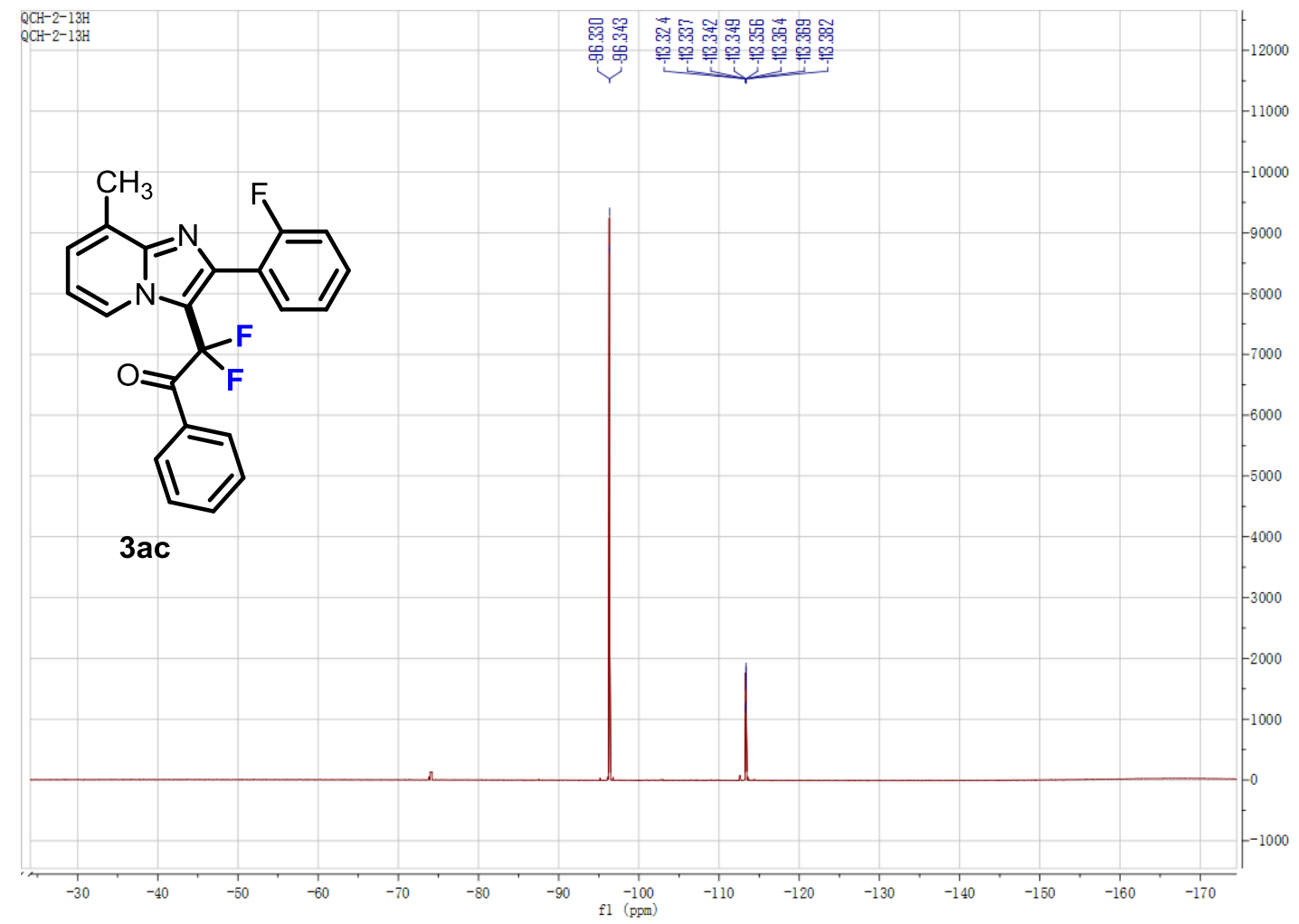

${ }^{13} \mathrm{C}$ NMR (100 MHz, $\left.\mathrm{CDCl}_{3}\right)$ :

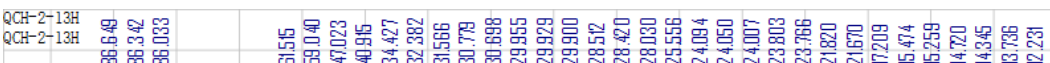

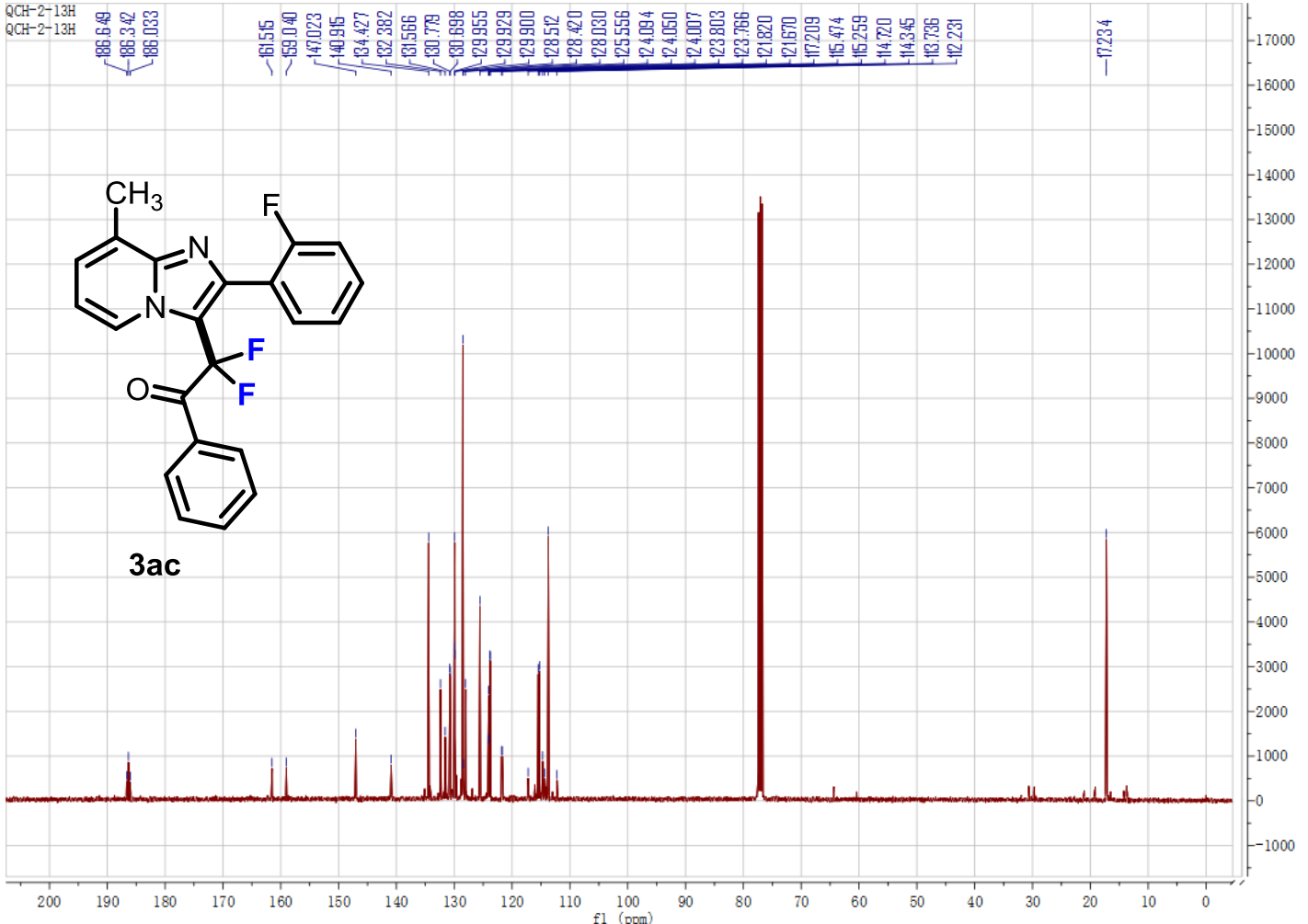


2-(8-bromo-2-phenylimidazo[1,2-a]pyridin-3-yl)-2,2-difluoro-1-phenylethan-1one (3ba)

${ }^{1} \mathrm{H}$ NMR (400 MHz, $\left.\mathrm{CDCl}_{3}\right)$ :

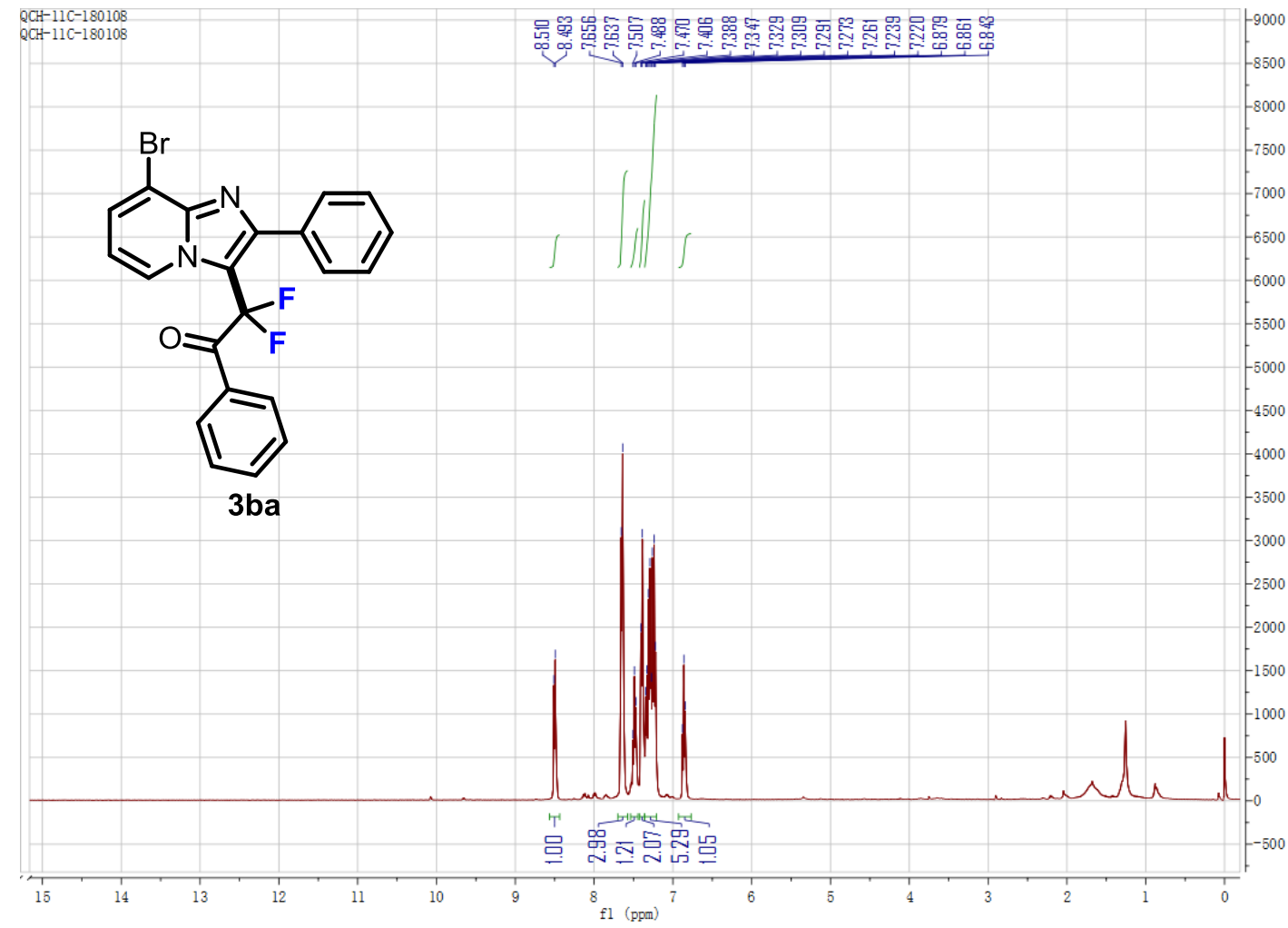


${ }^{19}$ F NMR (376 MHz, CDCl3):

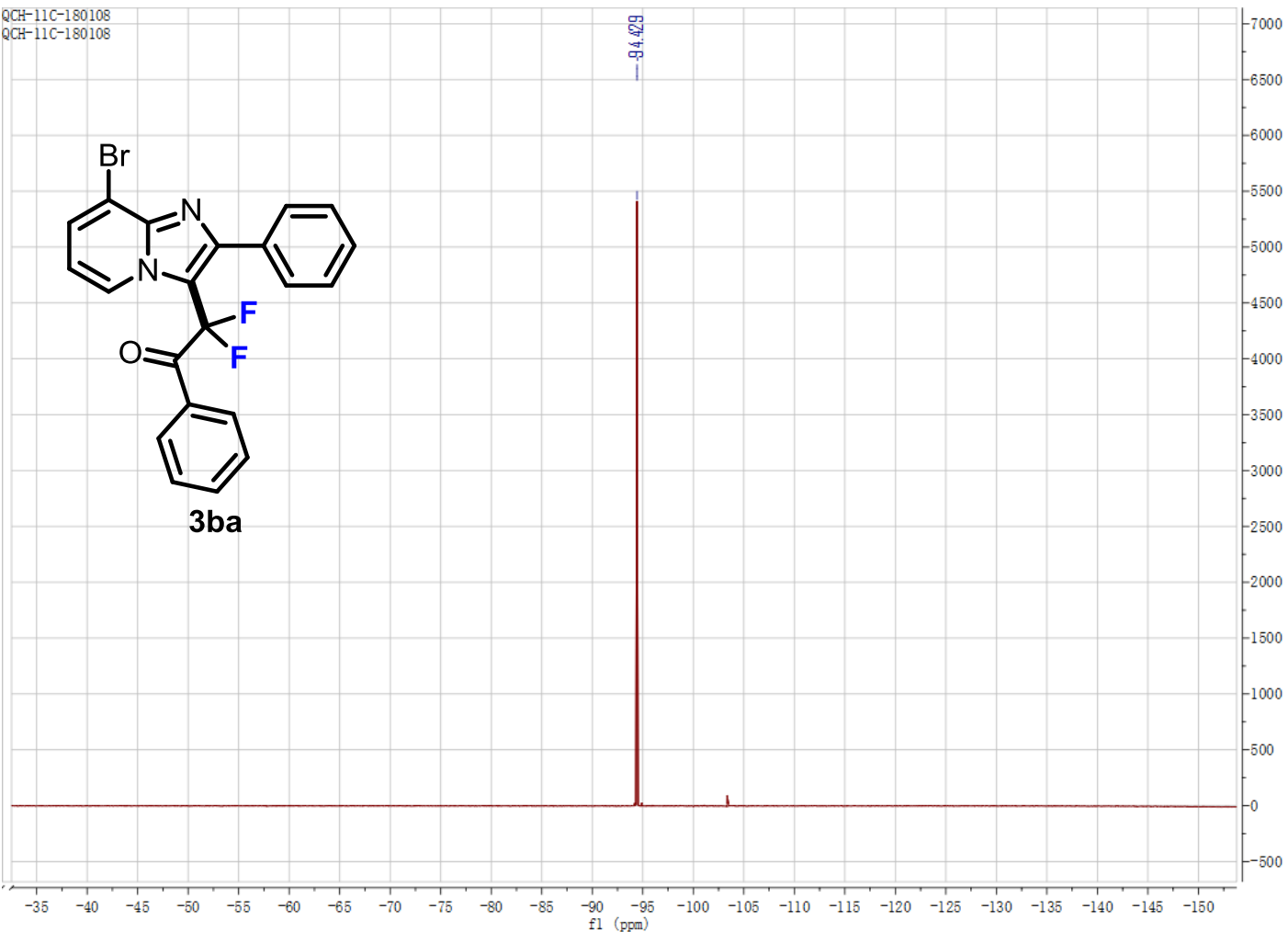

${ }^{13} \mathrm{C}$ NMR (100 MHz, CDCl 3$)$ :

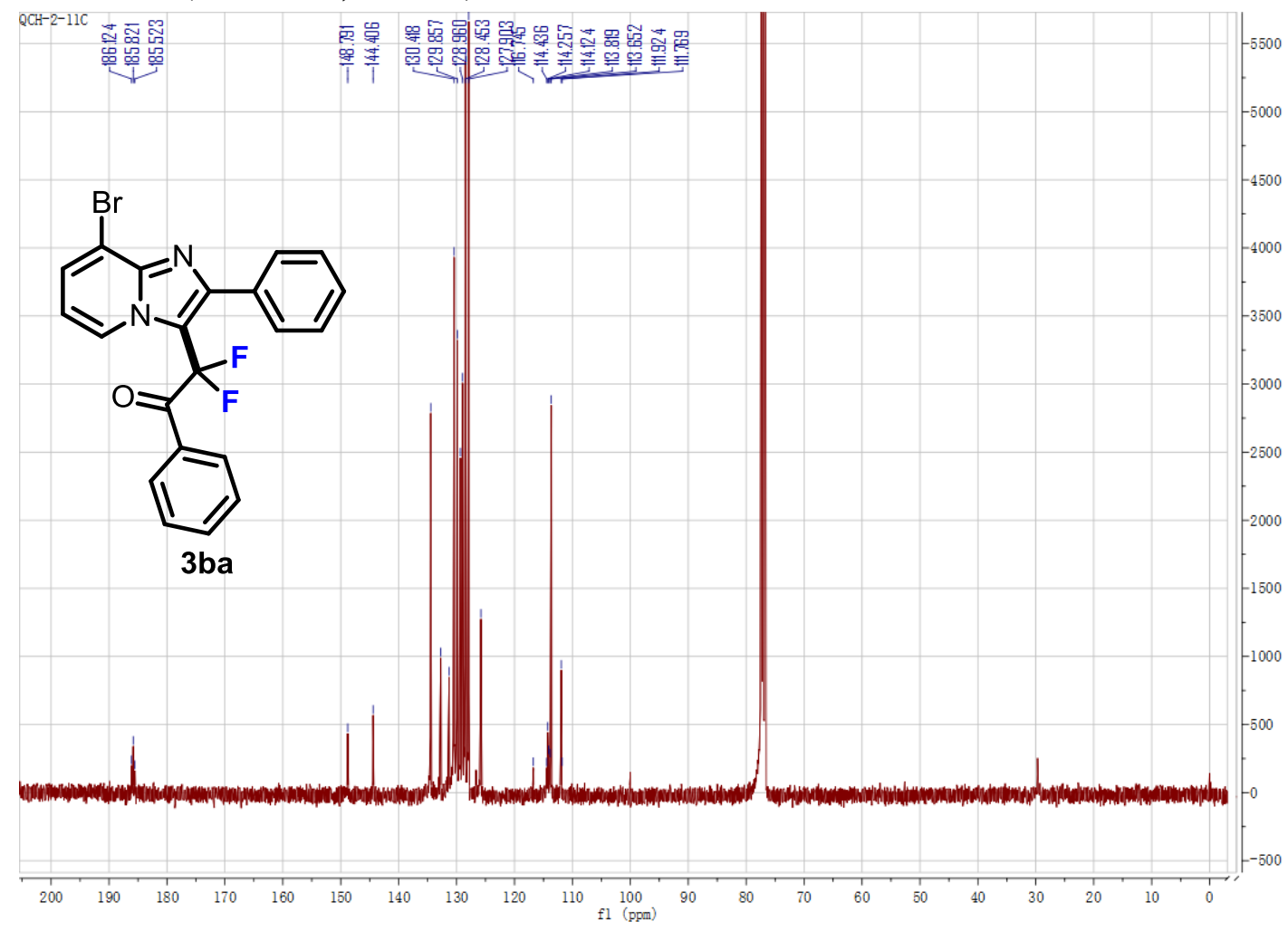


2-(8-bromo-2-(4-methoxyphenyl)imidazo[1,2-a]pyridin-3-yl)-2,2-difluoro-1-phenylethan $-1-$ one (3bb) ${ }^{1} \mathrm{H}$ NMR (400 MHz, $\left.\mathrm{CDCl}_{3}\right)$ :

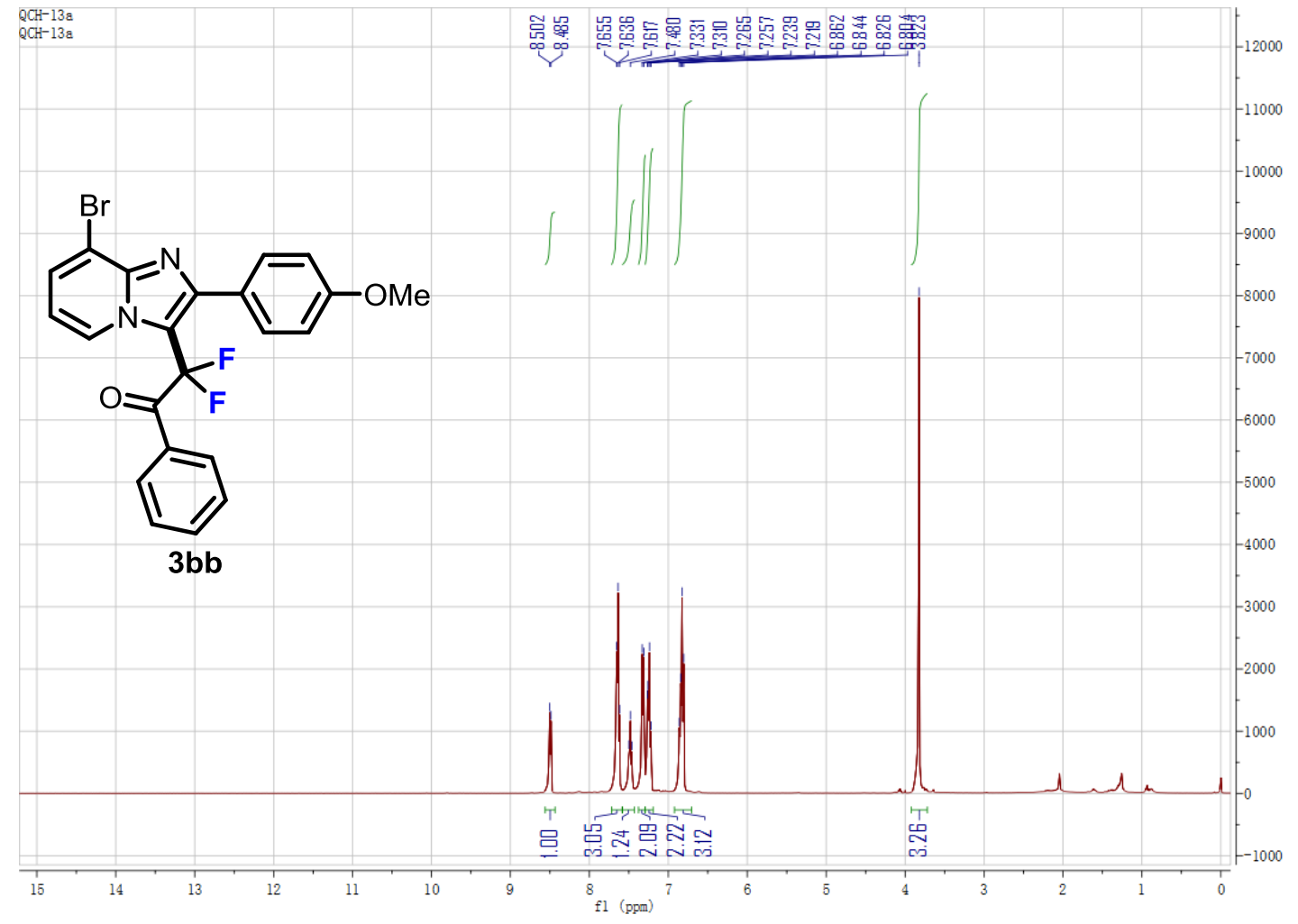


${ }^{19}$ F NMR (376 MHz, CDCl3):

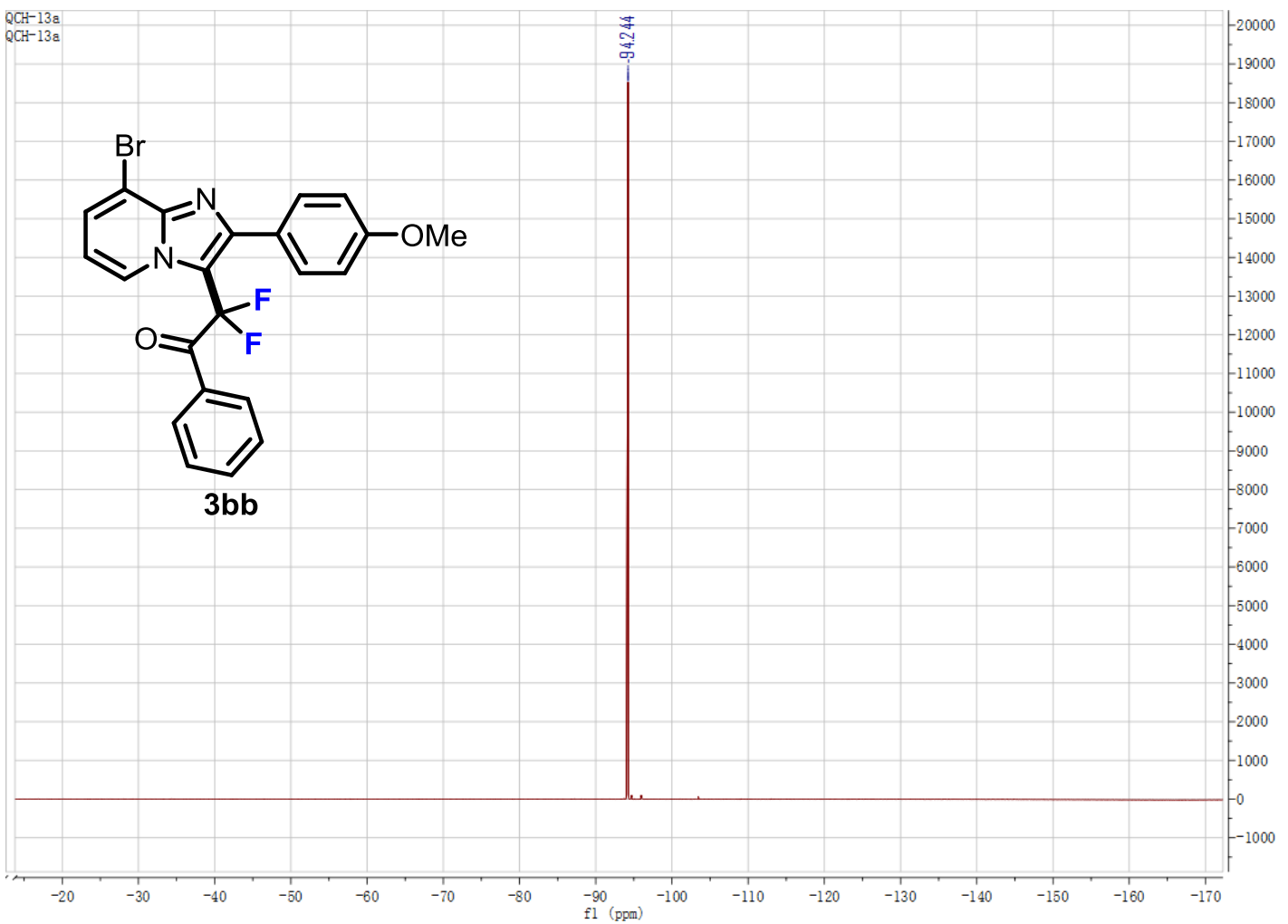

${ }^{3} \mathrm{C}$ NMR (100 MHz, CDCl3):

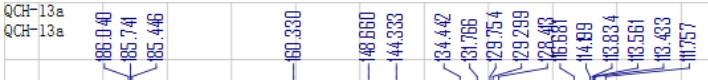<smiles>COc1ccc(-c2nc3c(Br)cccn3c2C(F)(F)C(=O)c2ccc(Br)cc2)cc1</smiles>
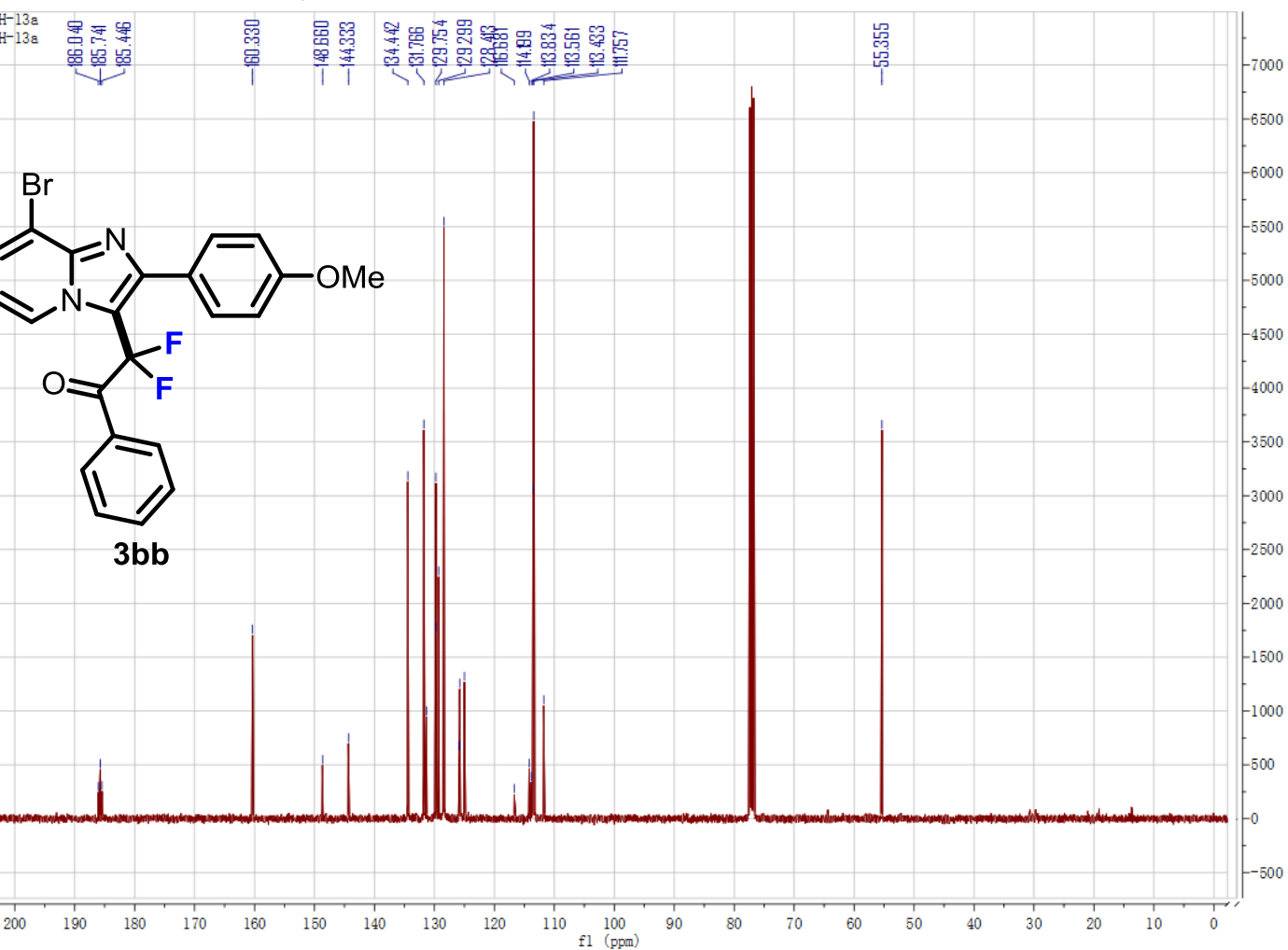
4-(8-bromo-3-(1,1-difluoro-2-oxo-2-phenylethyl)imidazo[1,2-a]pyridin-2-yl)benzonitrile (3bc)

${ }^{1} \mathrm{H}$ NMR (400 MHz, $\left.\mathrm{CDCl}_{3}\right)$ :

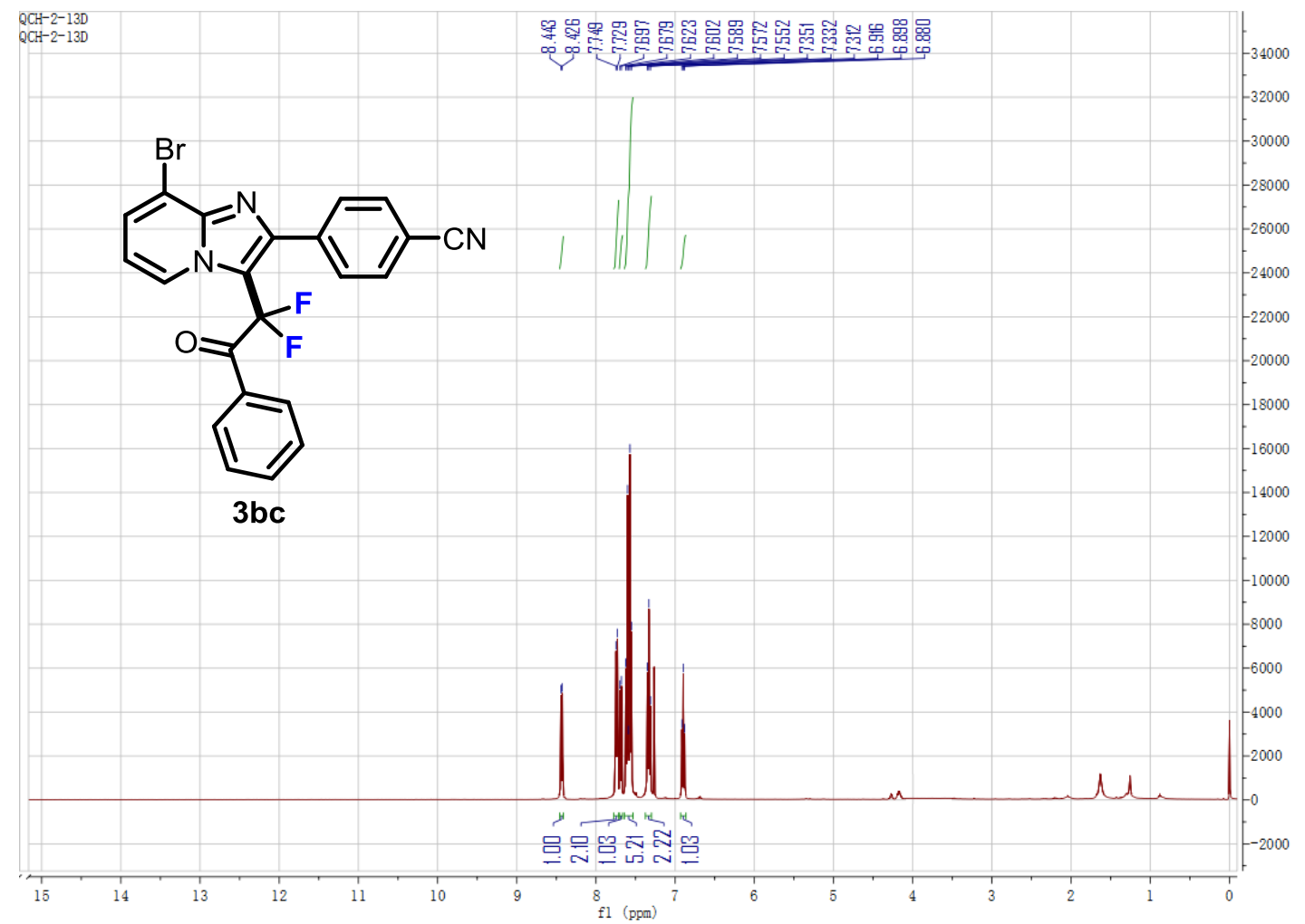


${ }^{19}$ F NMR (376 MHz, CDCl3):

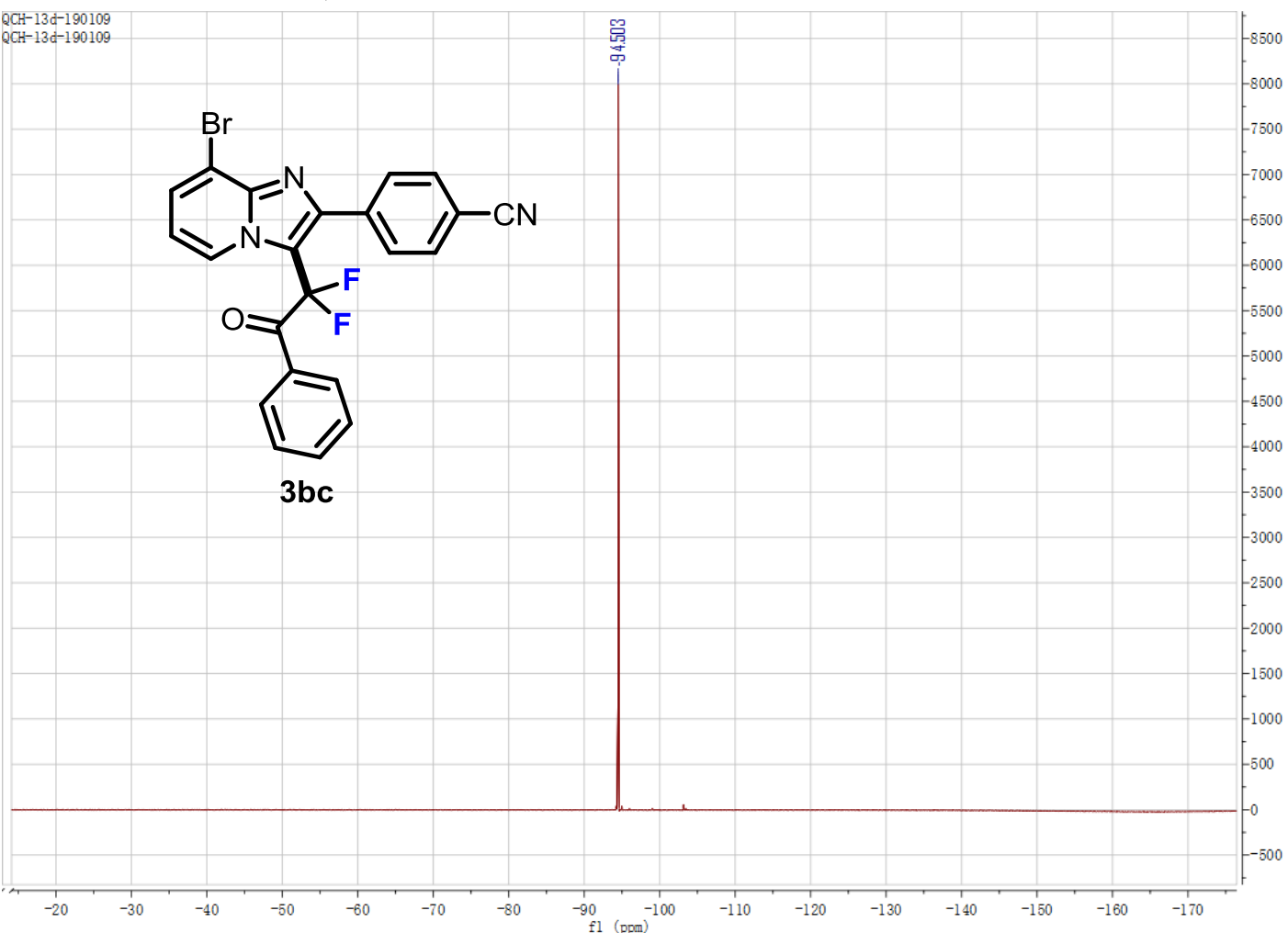

${ }^{13}$ C NMR (100 MHz, CDCl3):

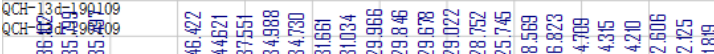

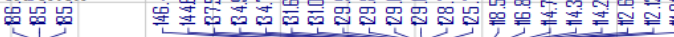<smiles>N#Cc1ccc(-c2nc3c(Br)cccn3c2C(F)(F)C(=O)c2ccccc2)cc1</smiles>

3bc
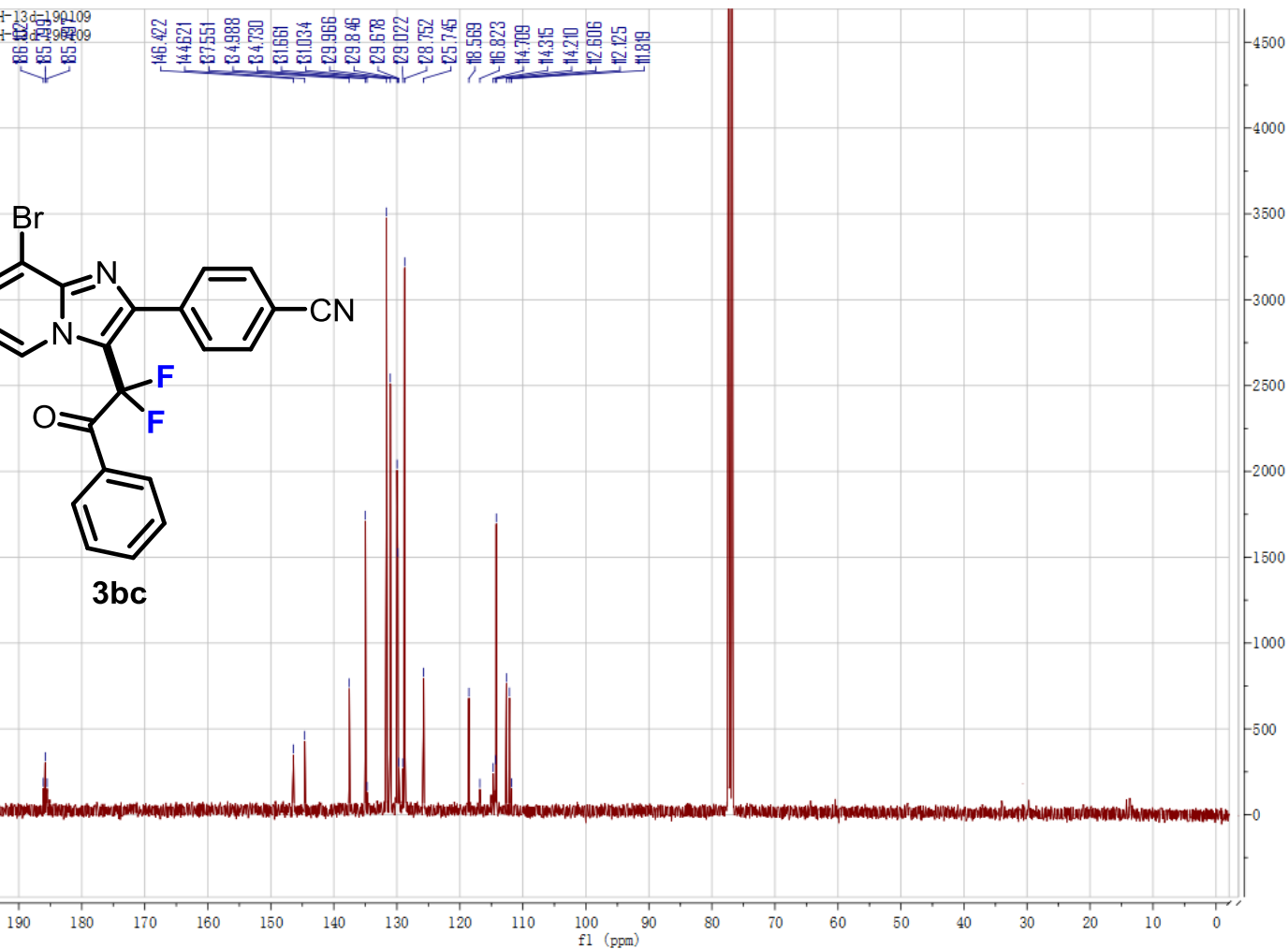
2-(8-bromo-2-(2-fluorophenyl)imidazo[1,2-a]pyridin-3-yl)-2,2-difluoro-1-phenylethan-1one (3bd)

${ }^{1} \mathrm{H}$ NMR (400 MHz, $\left.\mathrm{CDCl}_{3}\right)$ :

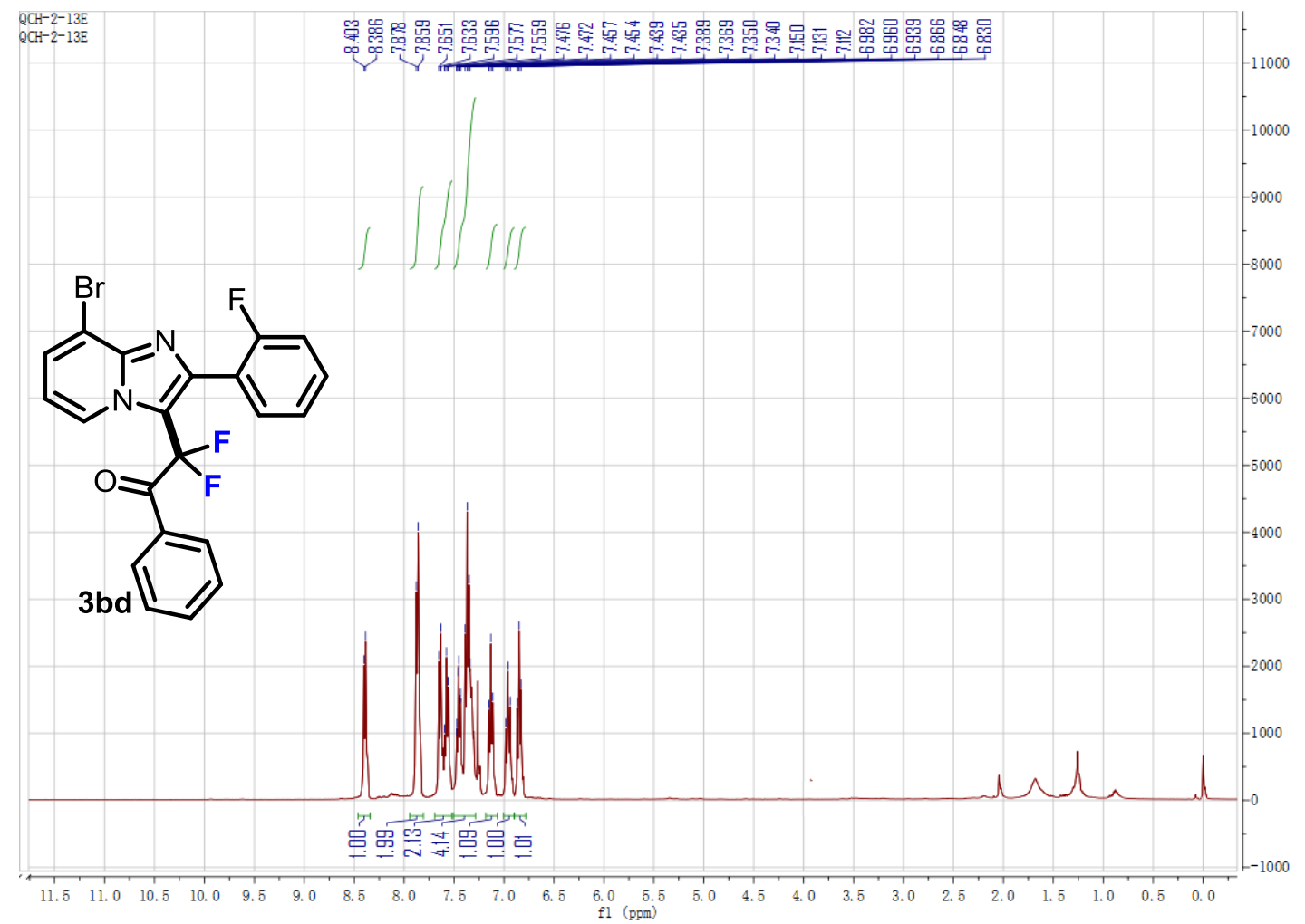


${ }^{19}$ F NMR (376 MHz, CDCl3):

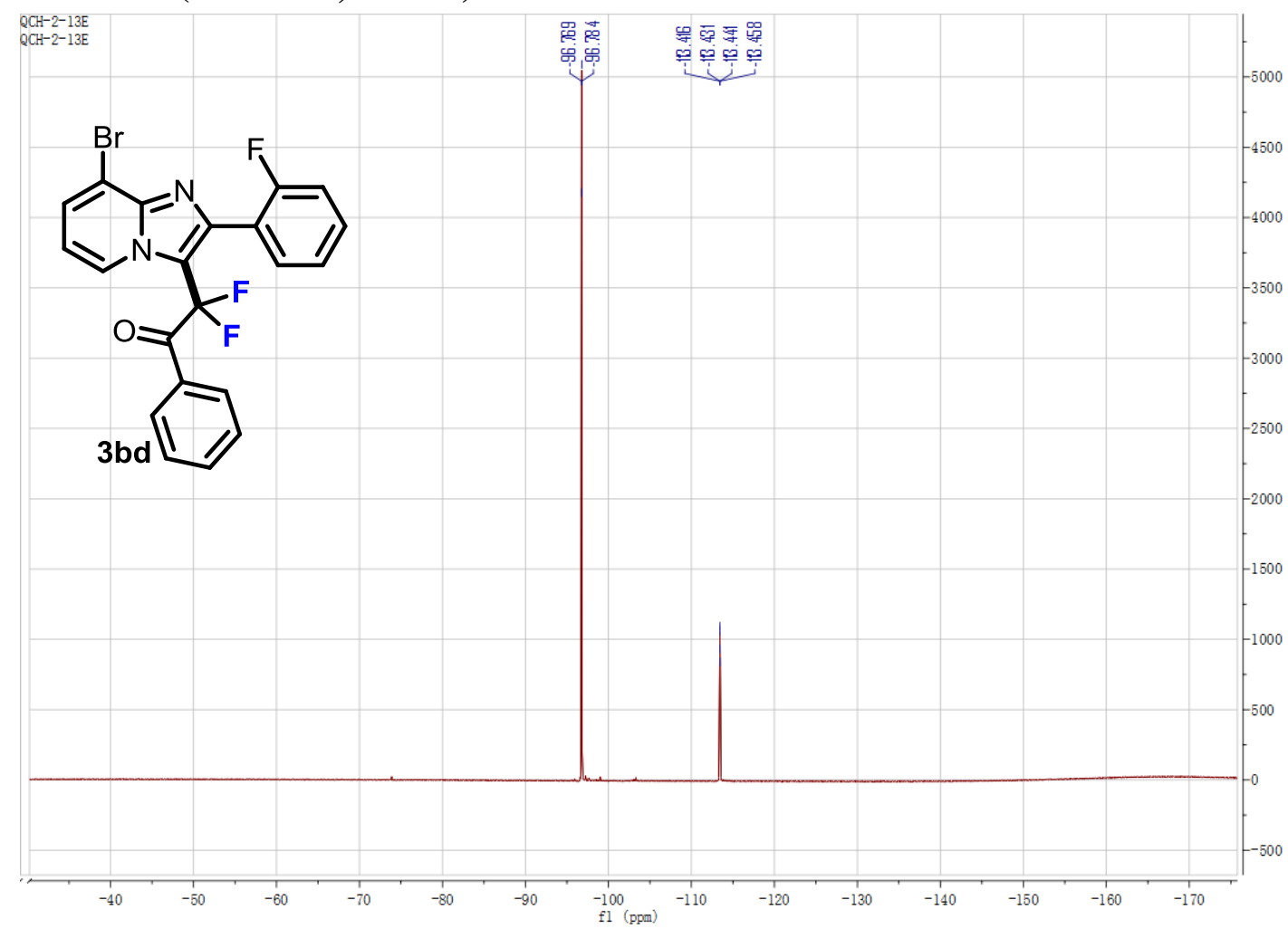

${ }^{13} \mathrm{C}$ NMR (100 MHz, CDCl 3$)$ :

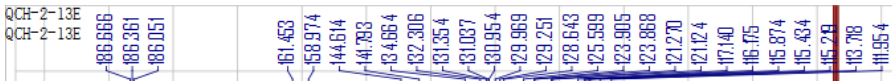

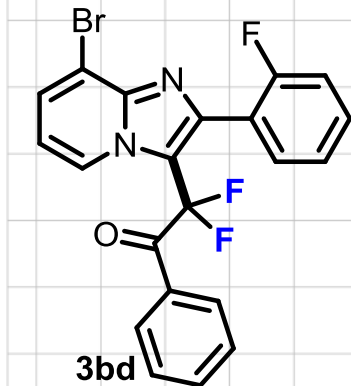


ethyl 8-bromo-3-(1,1-difluoro-2-oxo-2-phenylethyl)imidazo[1,2-a]pyridine-2carboxylate (3c)

${ }^{1} \mathrm{H}$ NMR (400 MHz, CDCl 3$)$ :

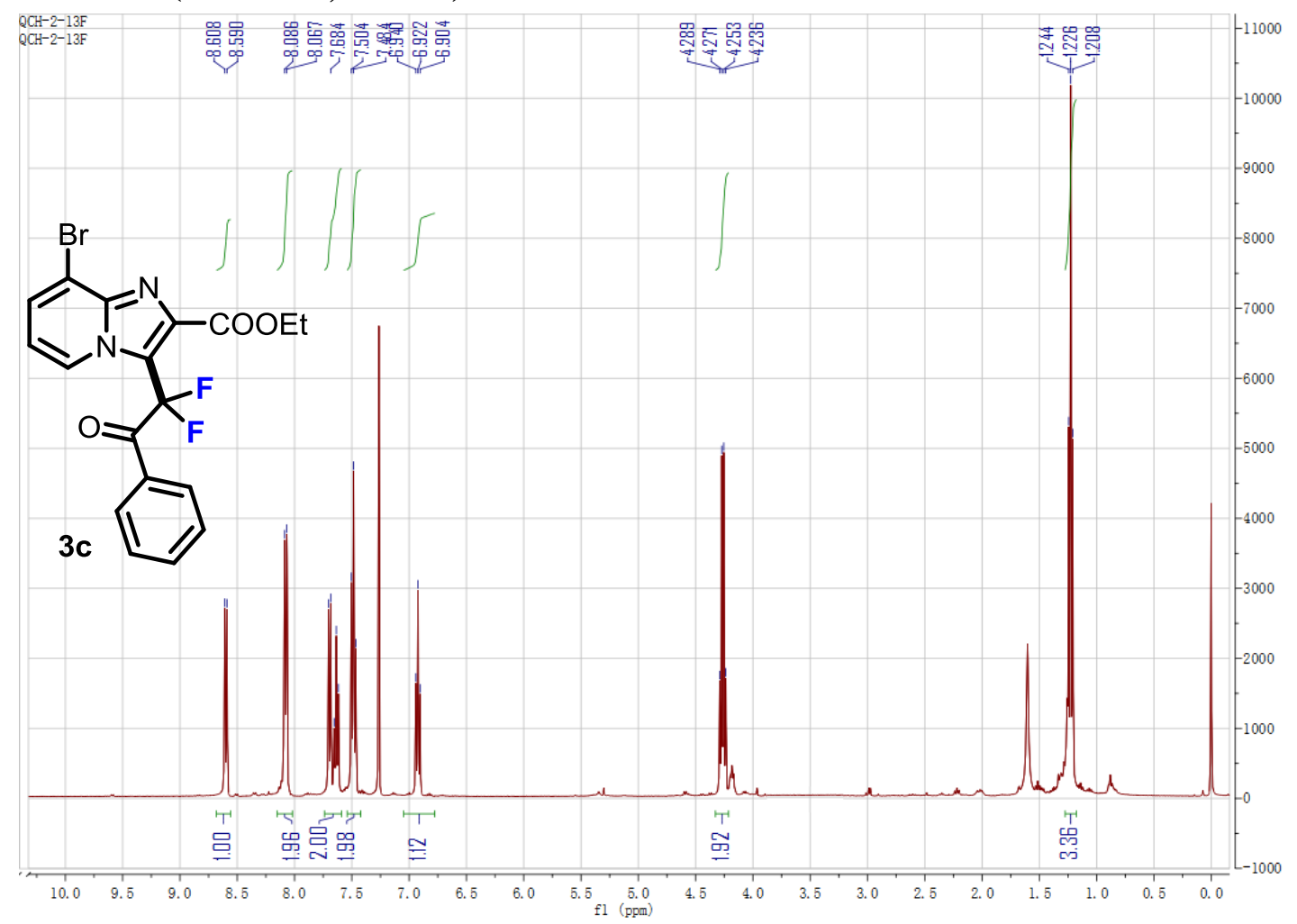


${ }^{19}$ F NMR (376 MHz, CDCl3):

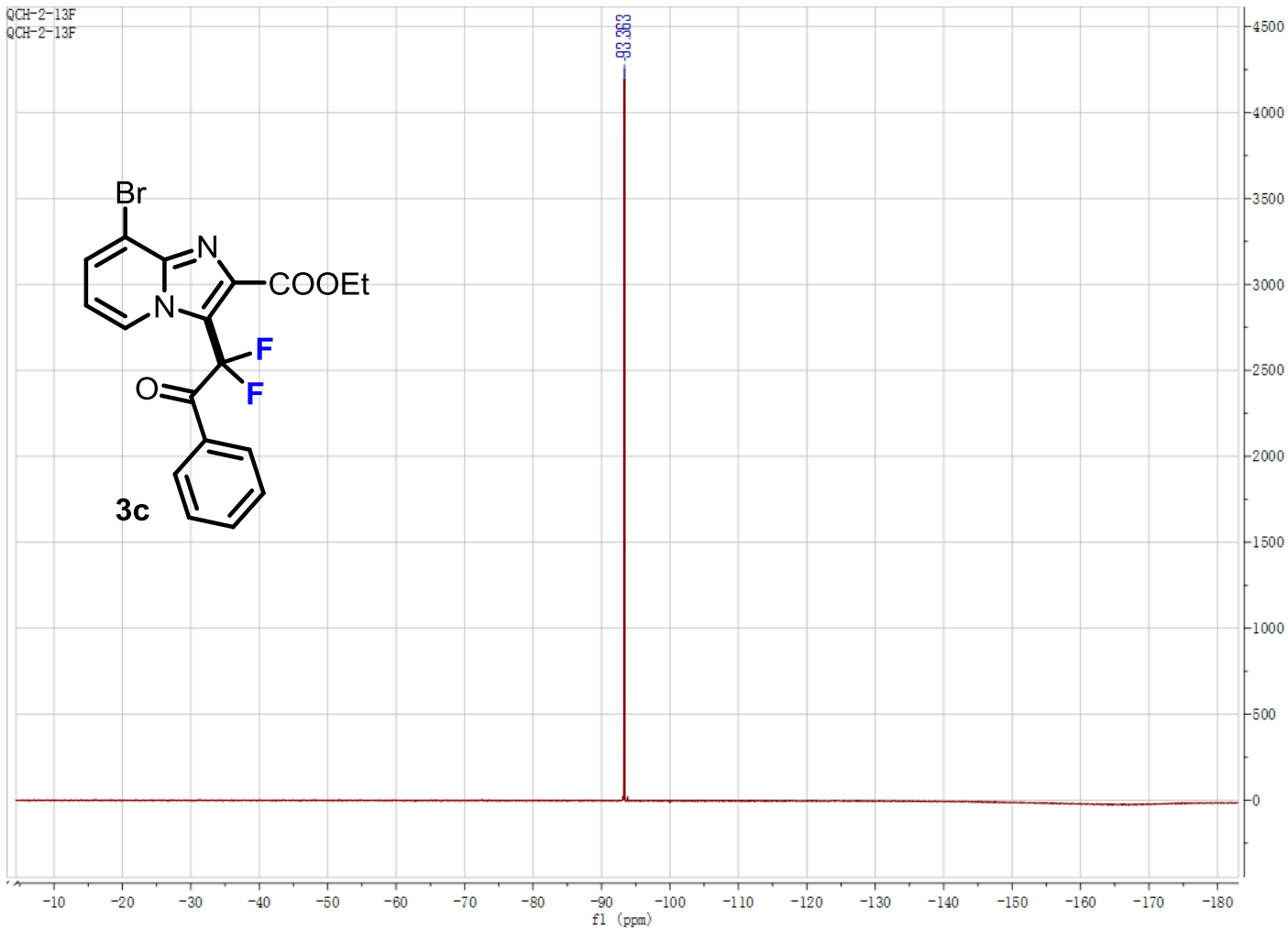

${ }^{13} \mathrm{C}$ NMR (100 MHz, CDCl 3$)$ :

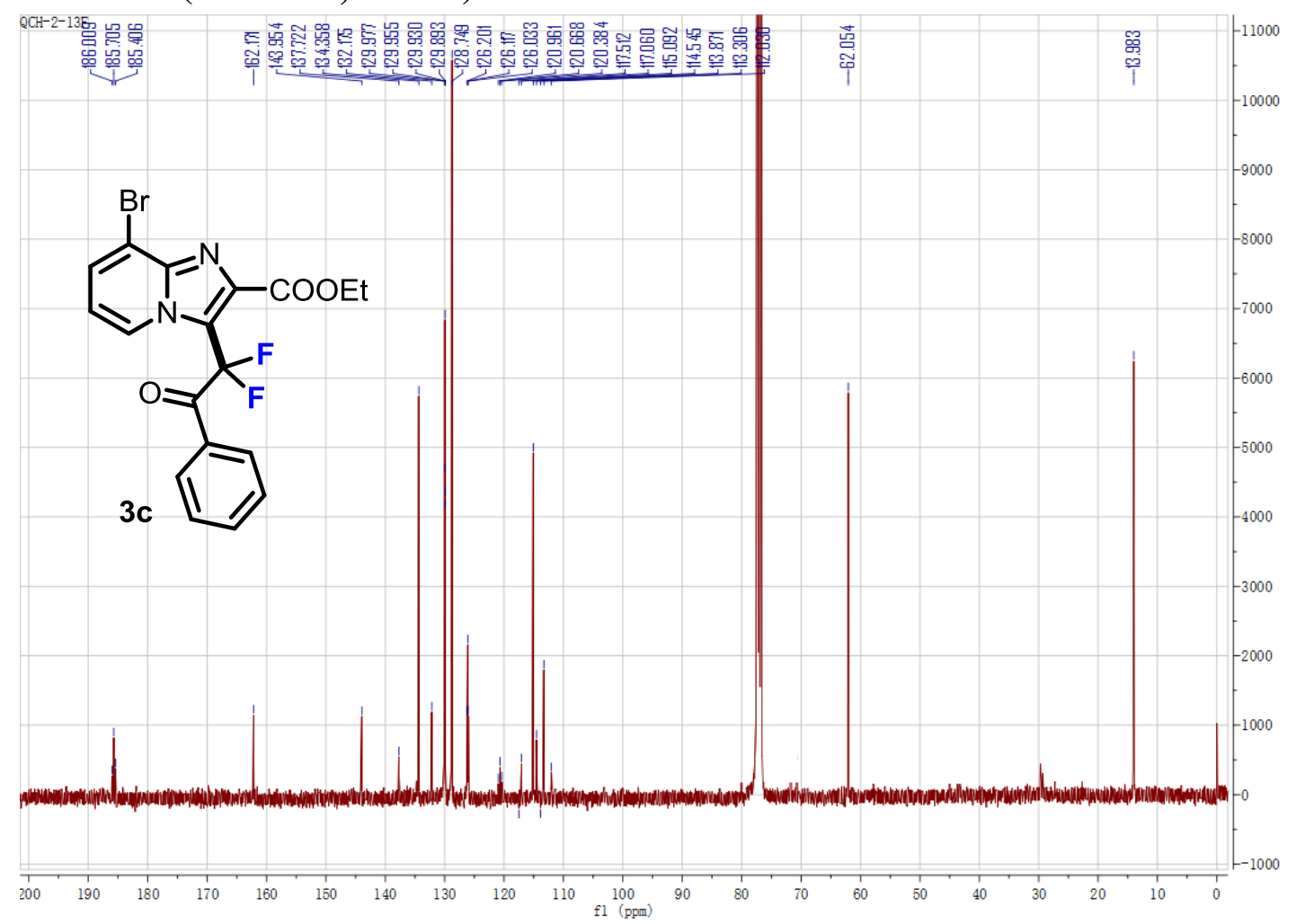


2-(7-chloro-2-(2-fluorophenyl)imidazo[1,2-a]pyridin-3-yl)-2,2-difluoro-1-phenylethan-1one (3d)

${ }^{1} \mathrm{H}$ NMR (400 MHz, CDCl3): 


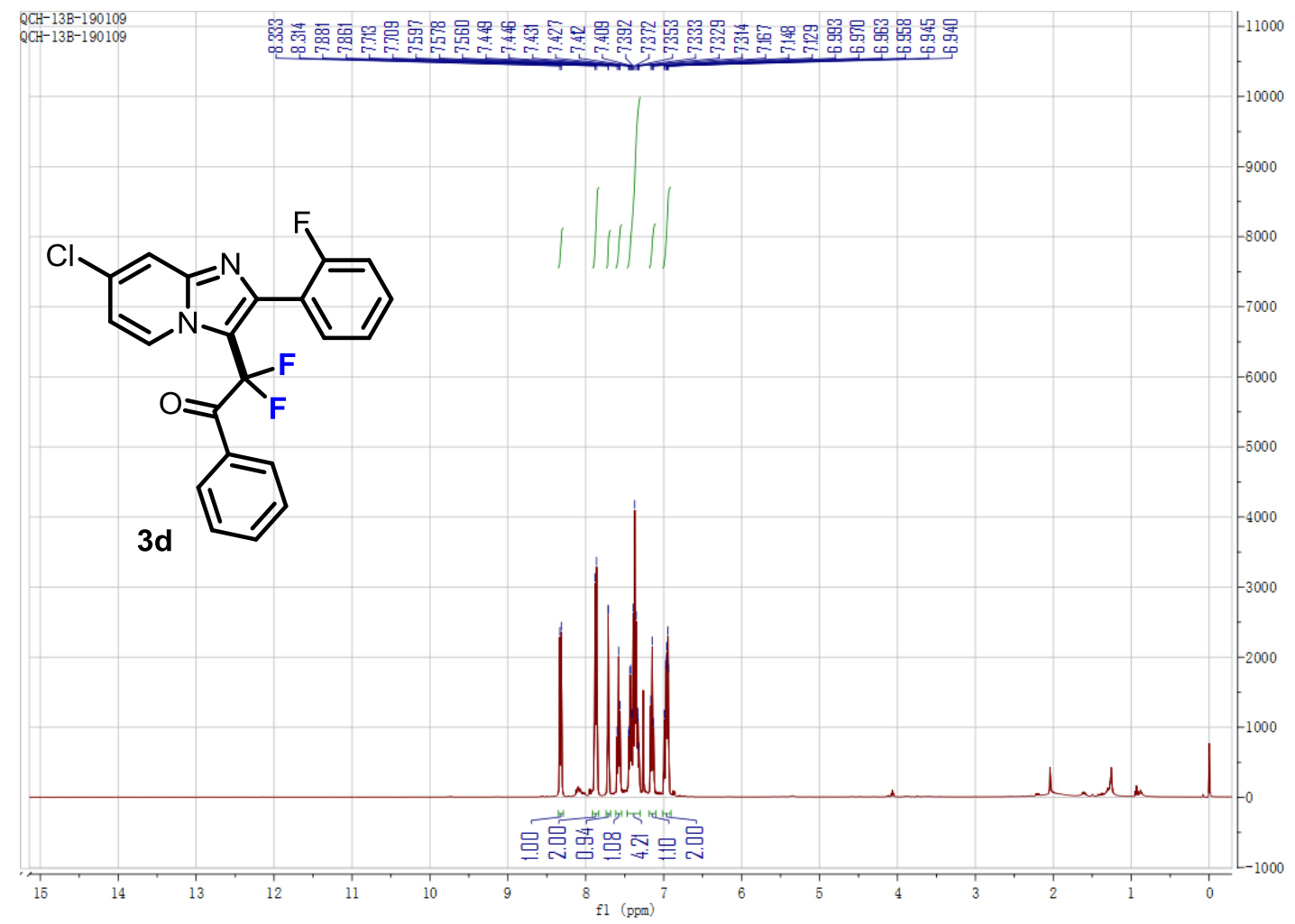

${ }^{19}$ F NMR (376 MHz, CDCl 3 ):

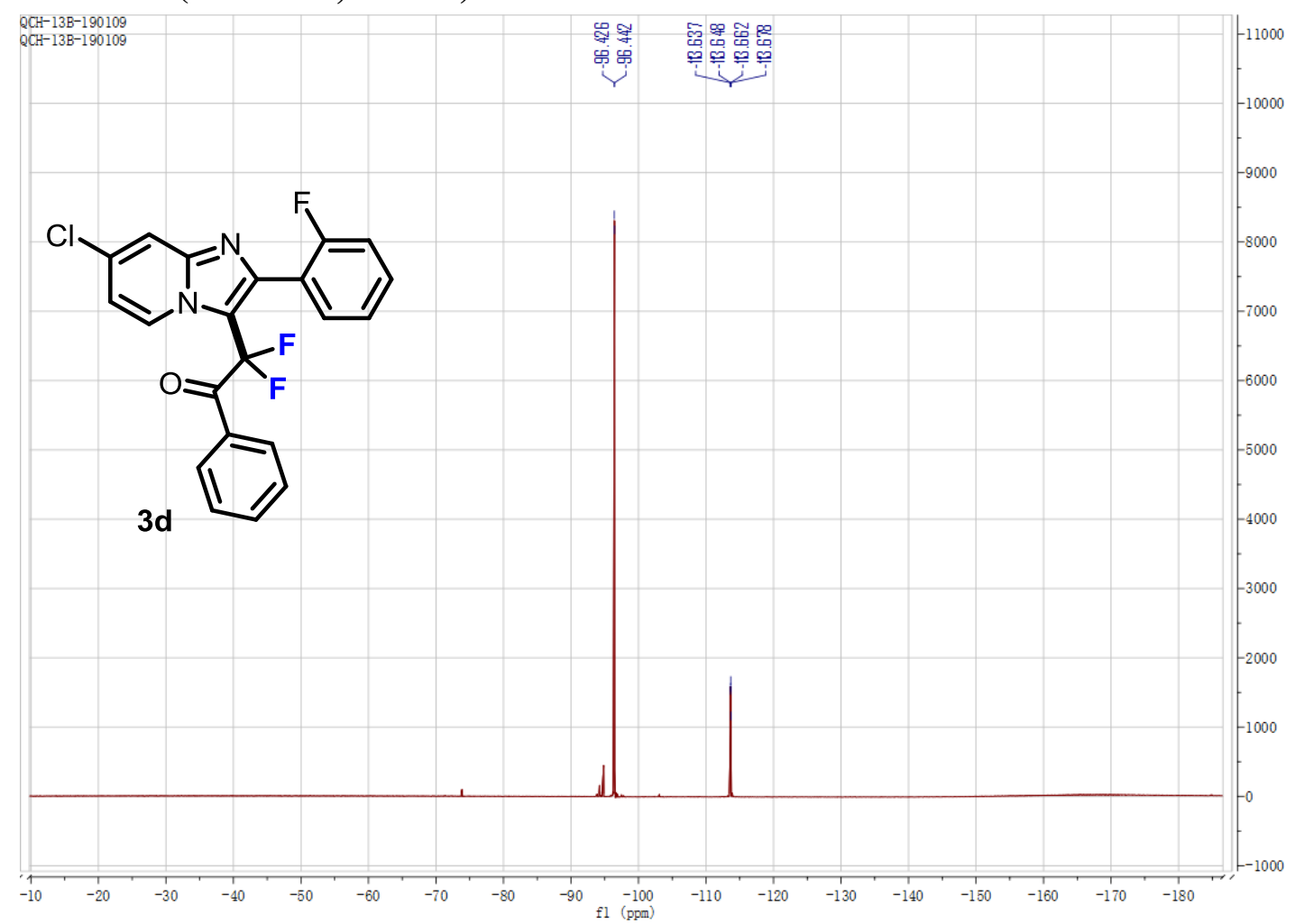

${ }^{13} \mathrm{C}$ NMR (100 MHz, CDCl3): 


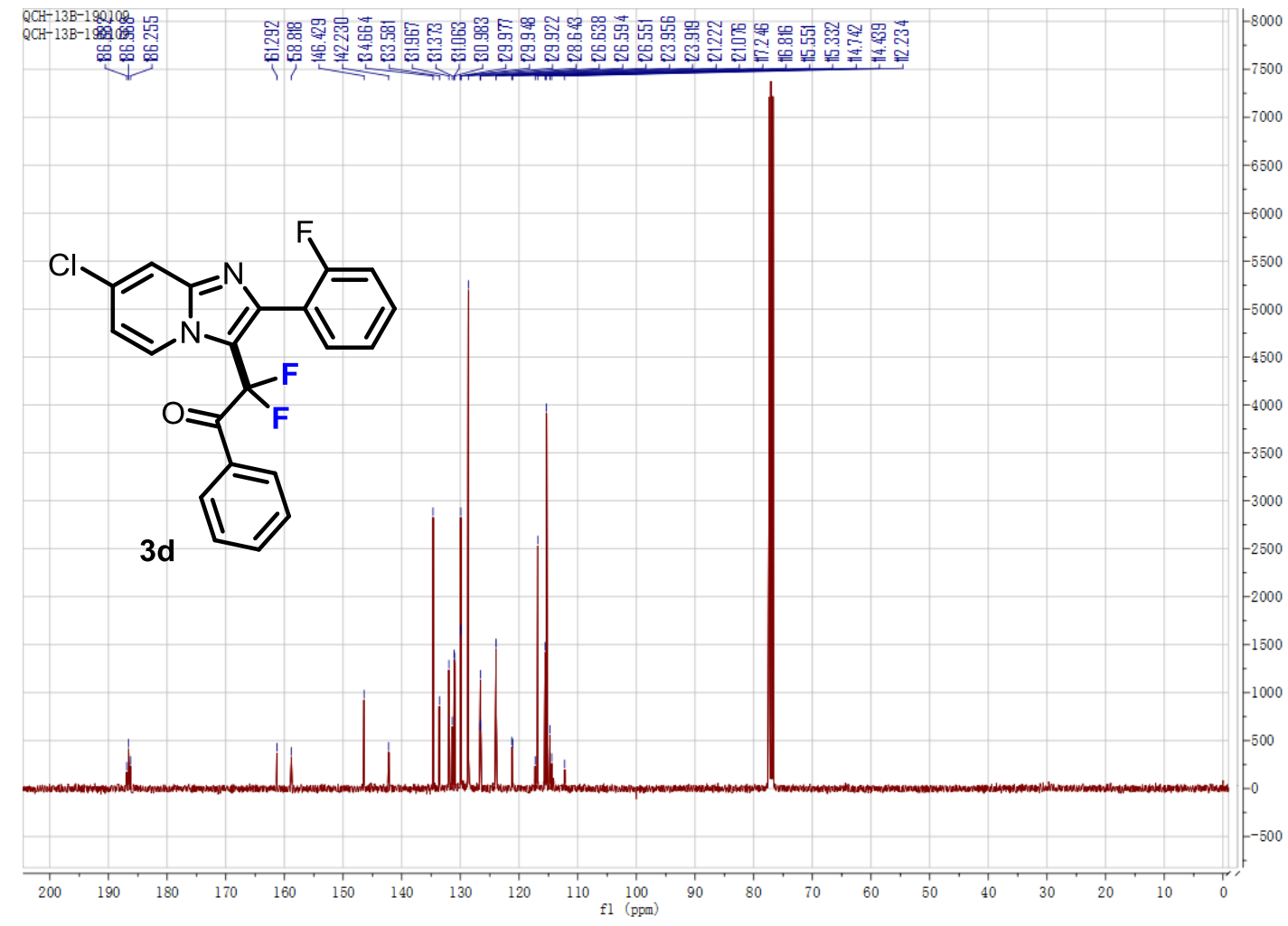


2-(8-bromo-6-methyl-2-phenylimidazo[1,2-a]pyridin-3-yl)-2,2-difluoro-1-phenylethan-1one (3ea)

${ }^{1} \mathrm{H}$ NMR (400 MHz, CDCl3):

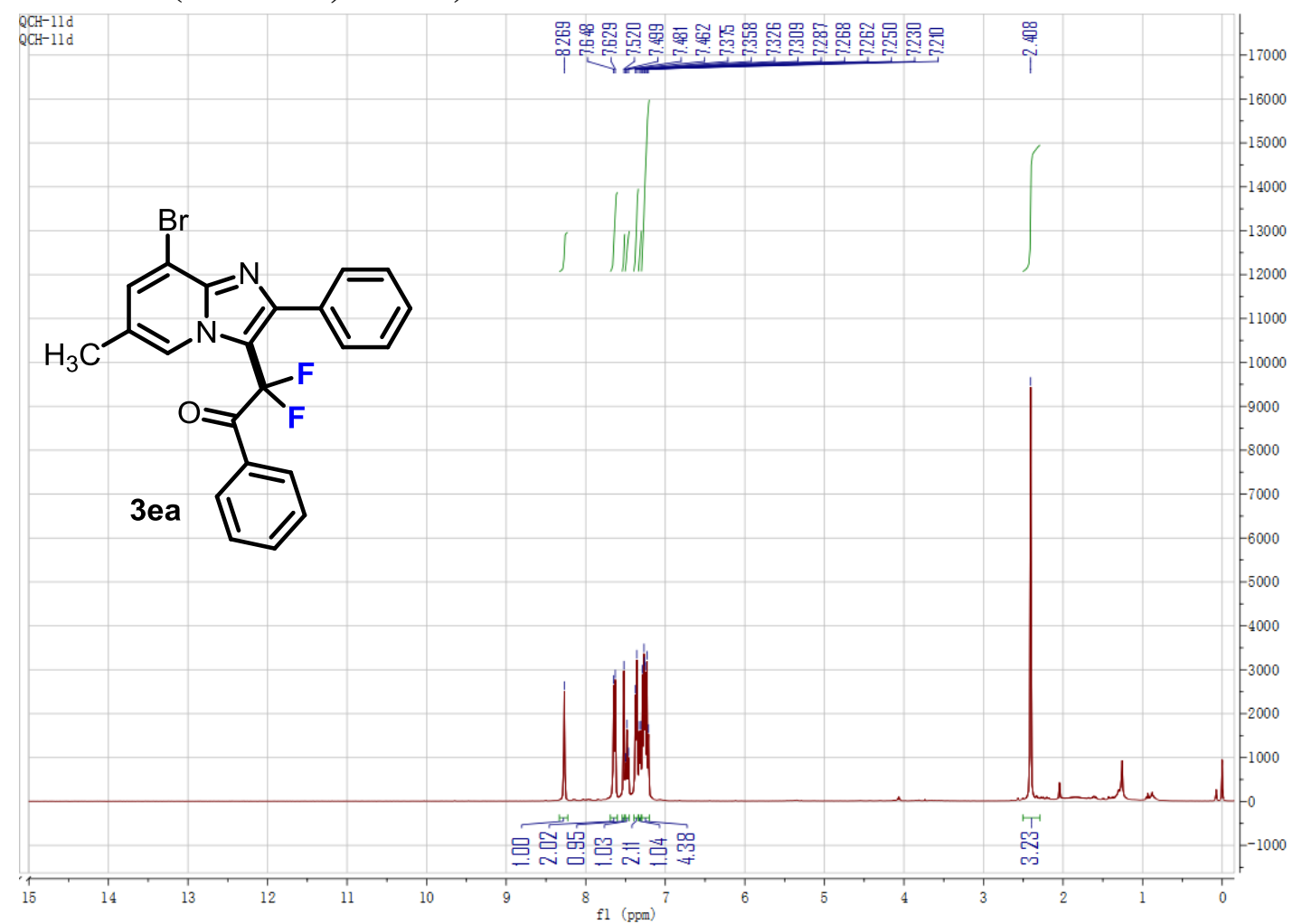

${ }^{19}$ F NMR (376 MHz, CDCl $)$ ):

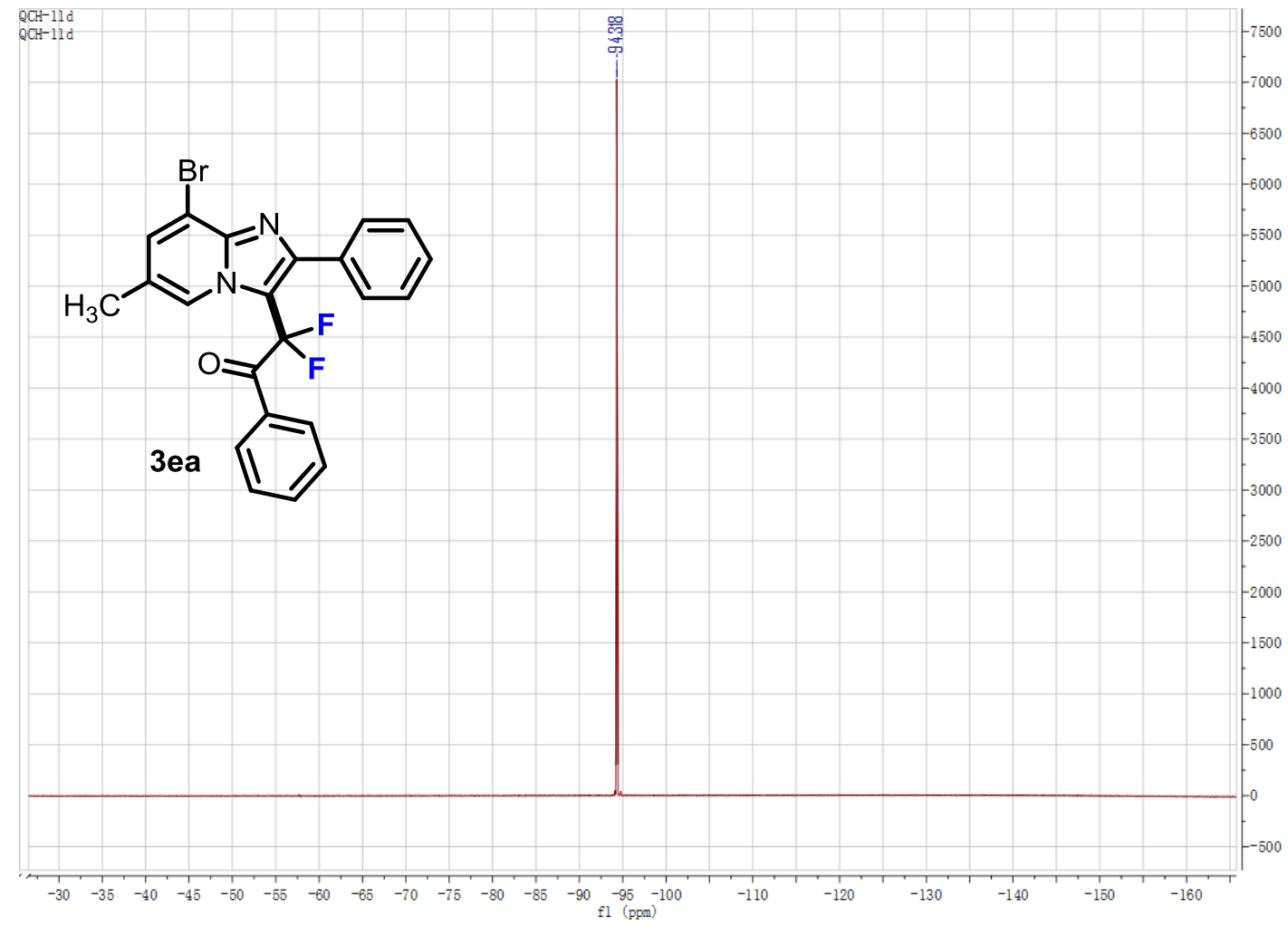


${ }^{13} \mathrm{C}$ NMR (100 MHz, CDCl3):

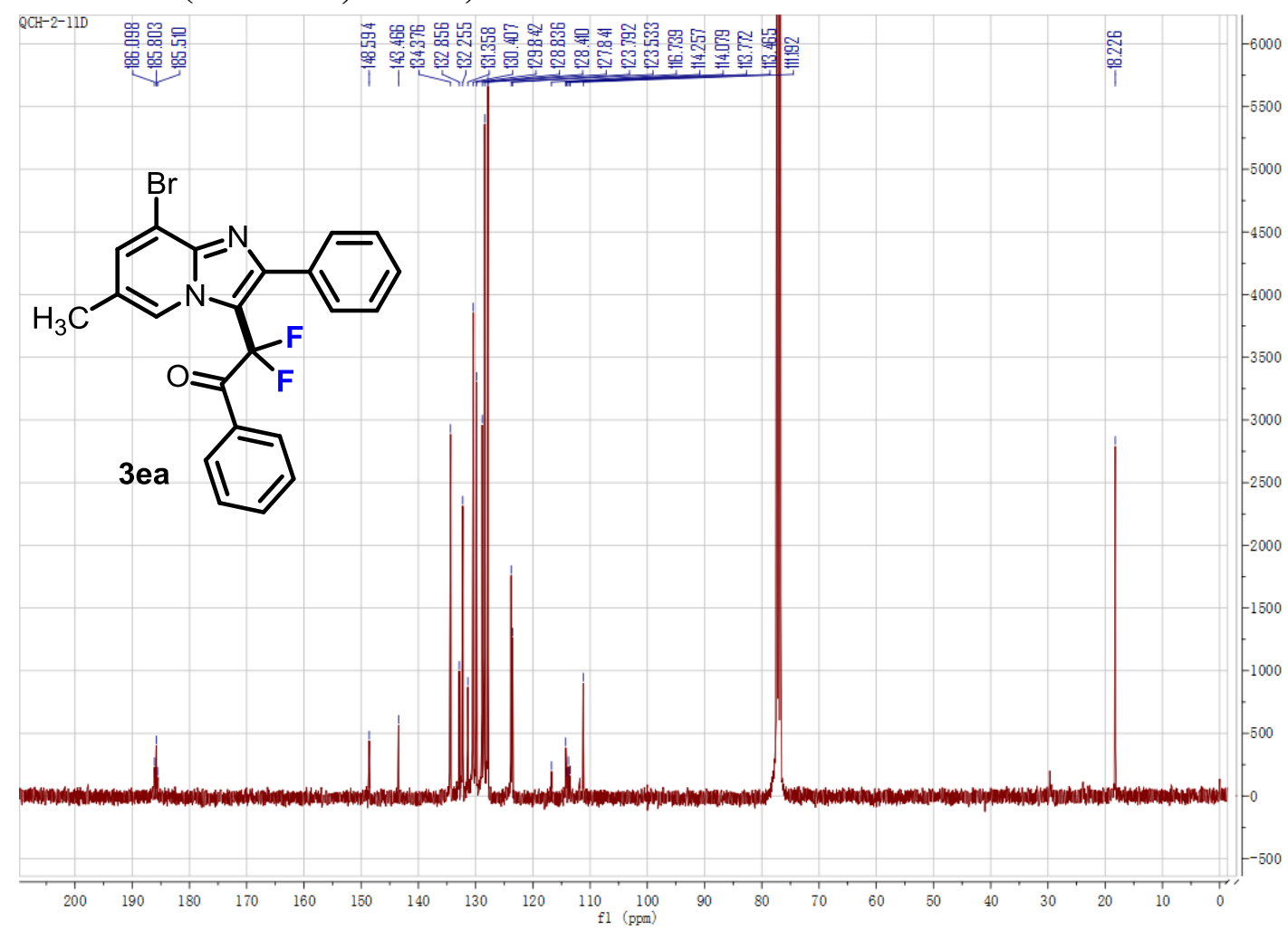


2-(8-bromo-2-(4-methoxyphenyl)-6-methylimidazo[1,2-a]pyridin-3-yl)-2,2-difluoro-1-ph enylethan-1-one (3eb)

${ }^{1}$ H NMR (400 MHz, CDCl3):

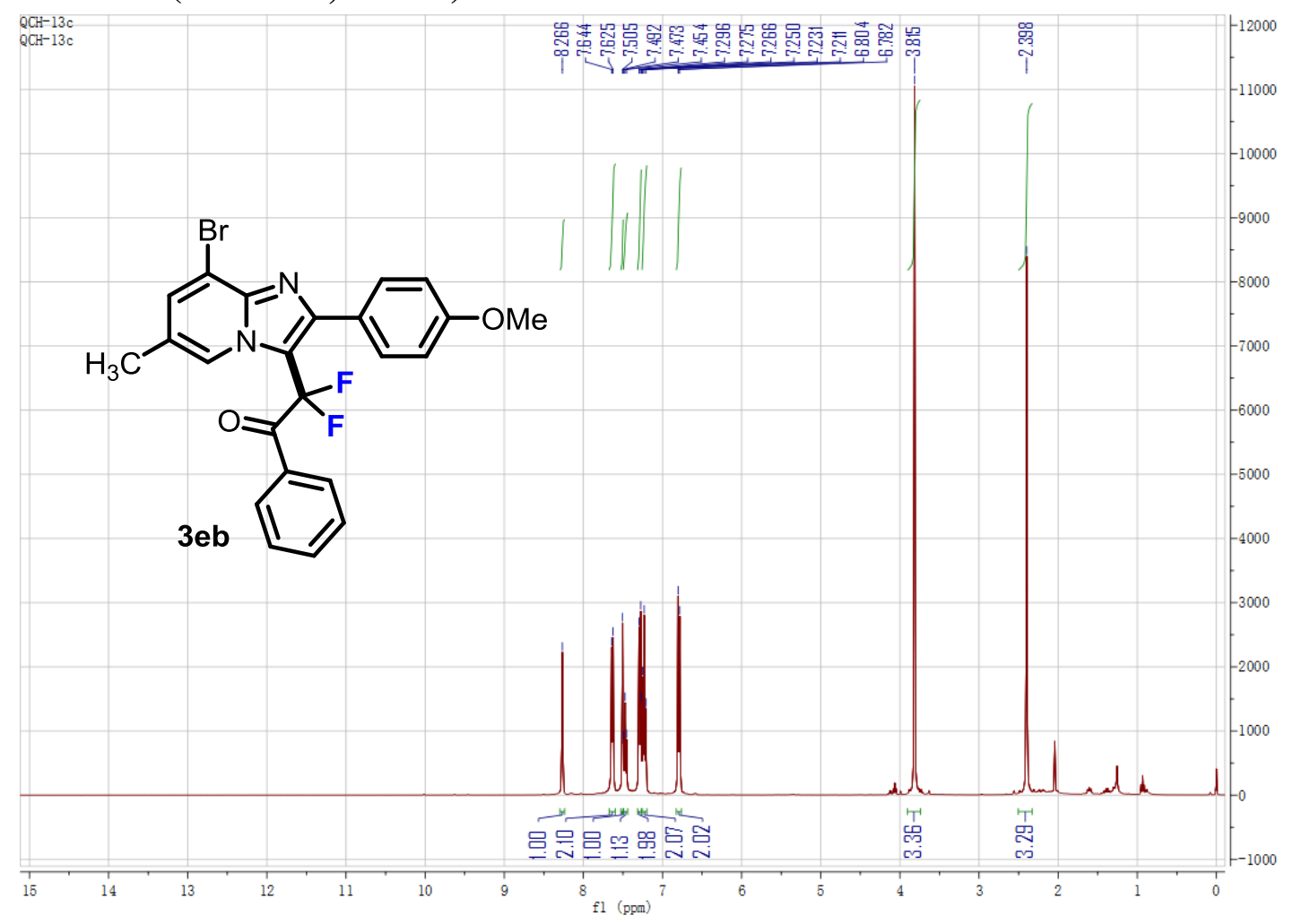

${ }^{19}$ F NMR (376 MHz, CDCl3):

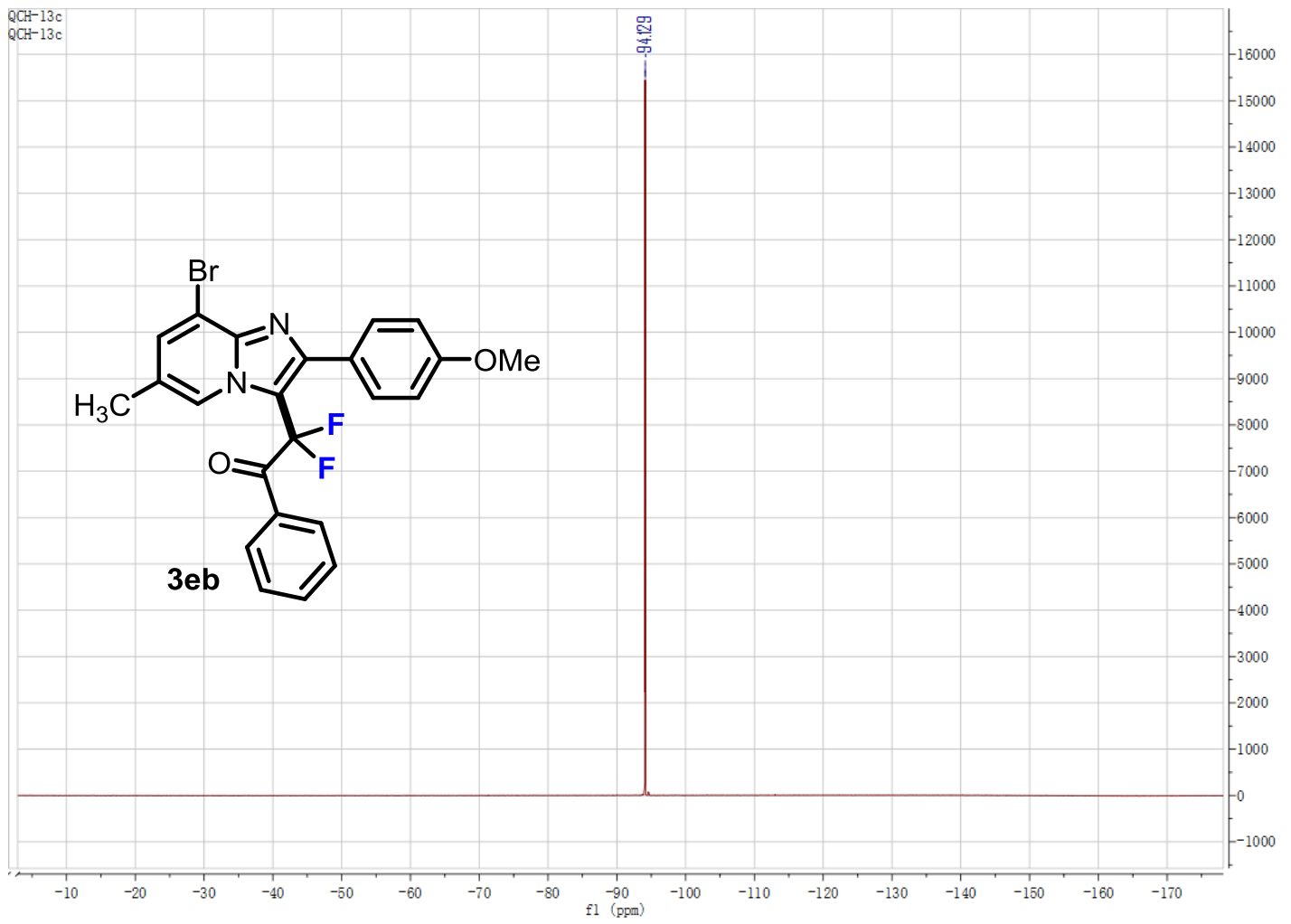


${ }^{13} \mathrm{C}$ NMR (100 MHz, CDCl3):

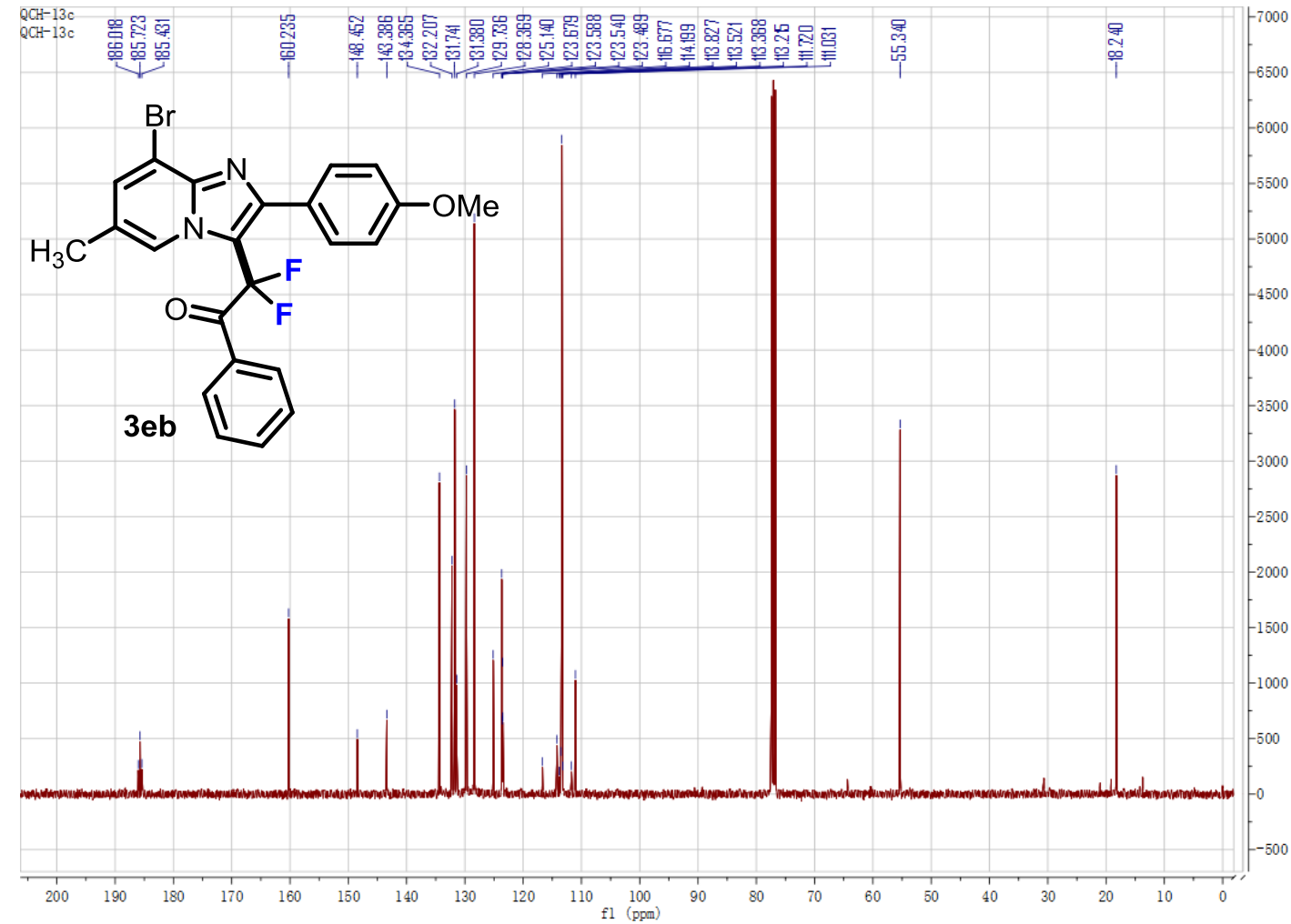


2,2-difluoro-1-phenyl-2-(6-phenylimidazo[2,1-b]thiazol-5-yl)ethan-1-one (3fa) ${ }^{1}$ H NMR (400 MHz, CDCl3):

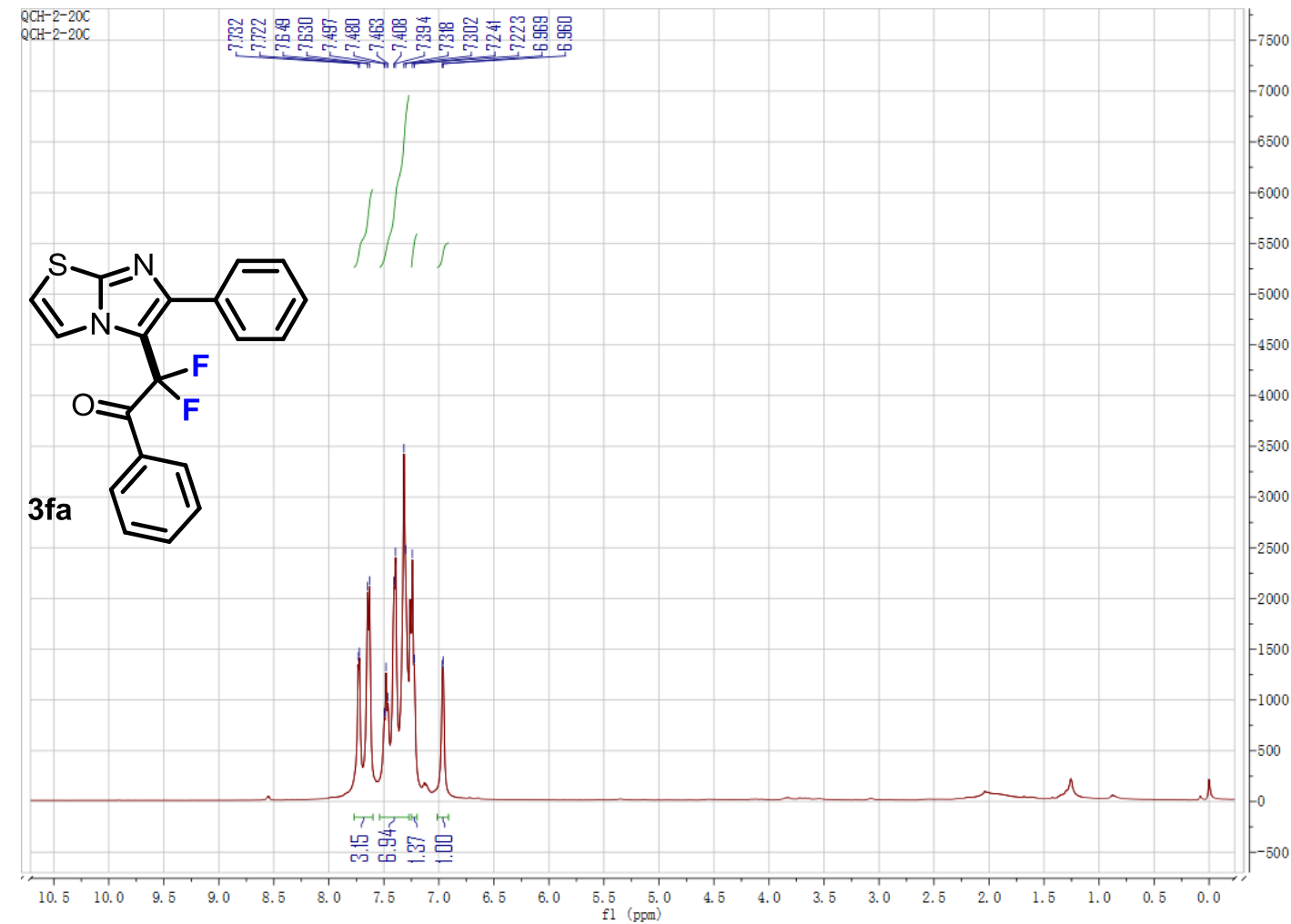

${ }^{19}$ F NMR (376 MHz, CDCl$)$ :

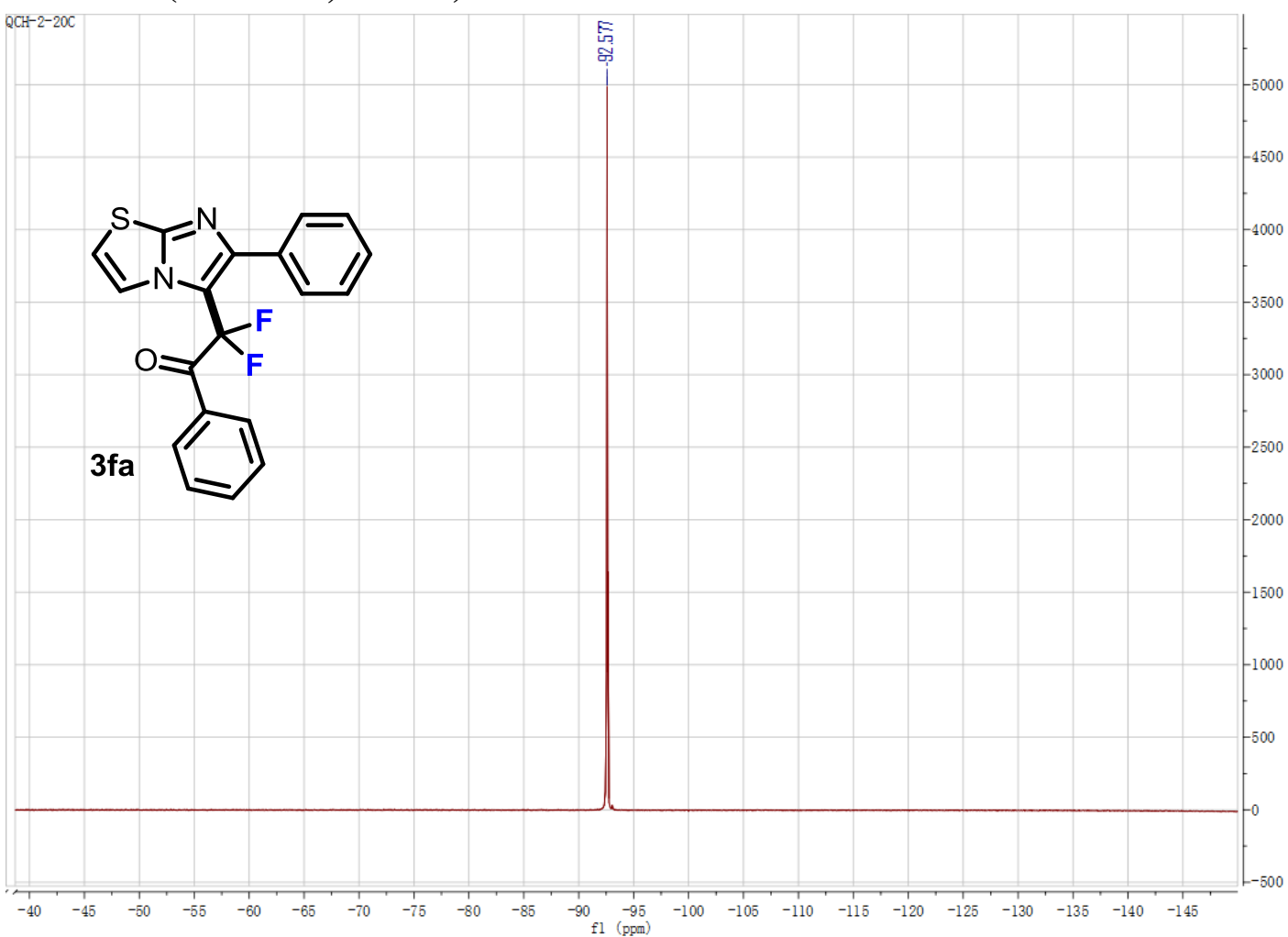


${ }^{13} \mathrm{C}$ NMR (100 MHz, $\left.\mathrm{CDCl}_{3}\right)$ :

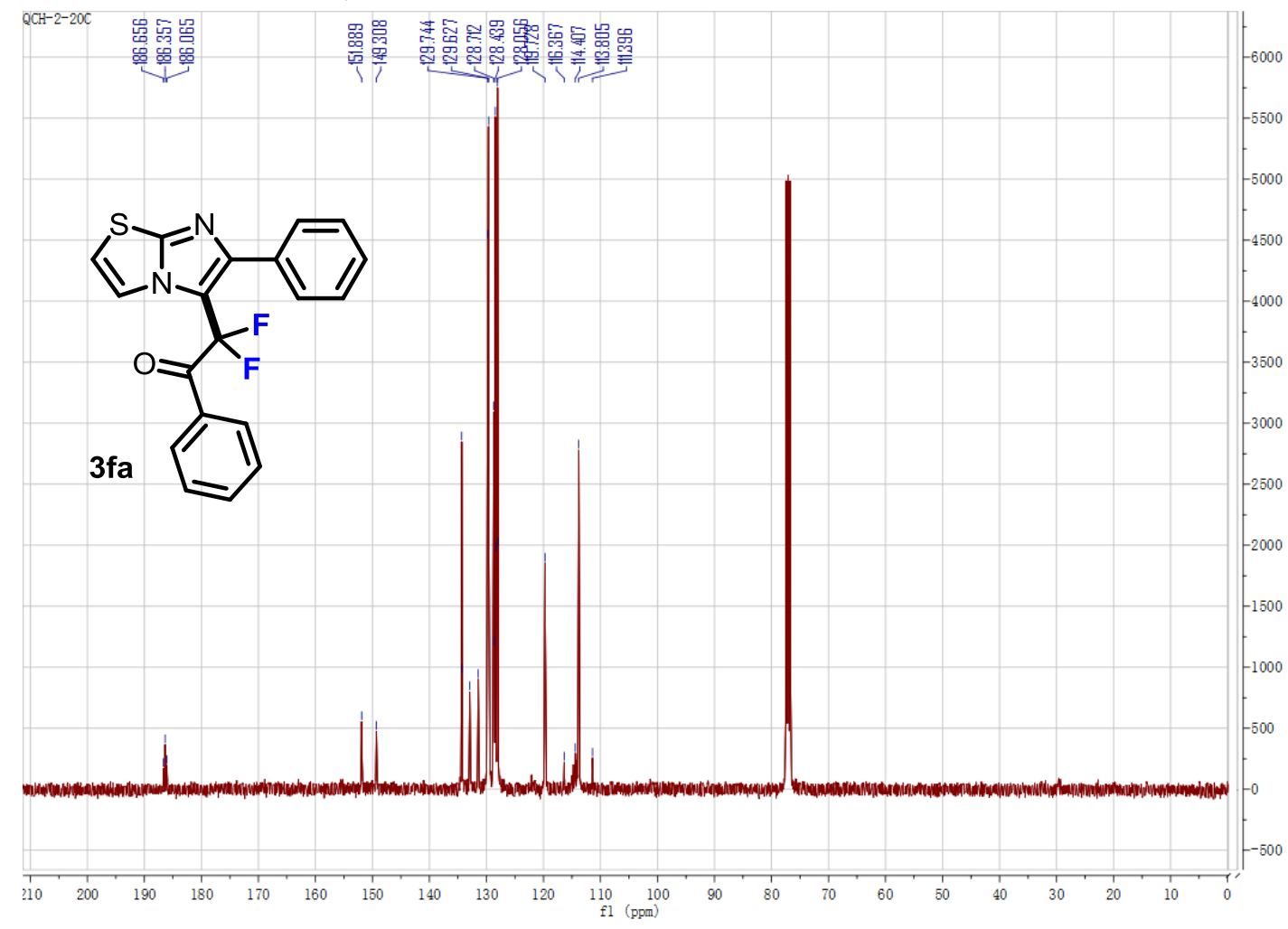


2,2-difluoro-2-(6-(4-methoxyphenyl)imidazo[2,1-b]thiazol-5-yl)-1-phenylethan-1-one (3fb)

${ }^{1} \mathrm{H}$ NMR (400 MHz, CDCl3):

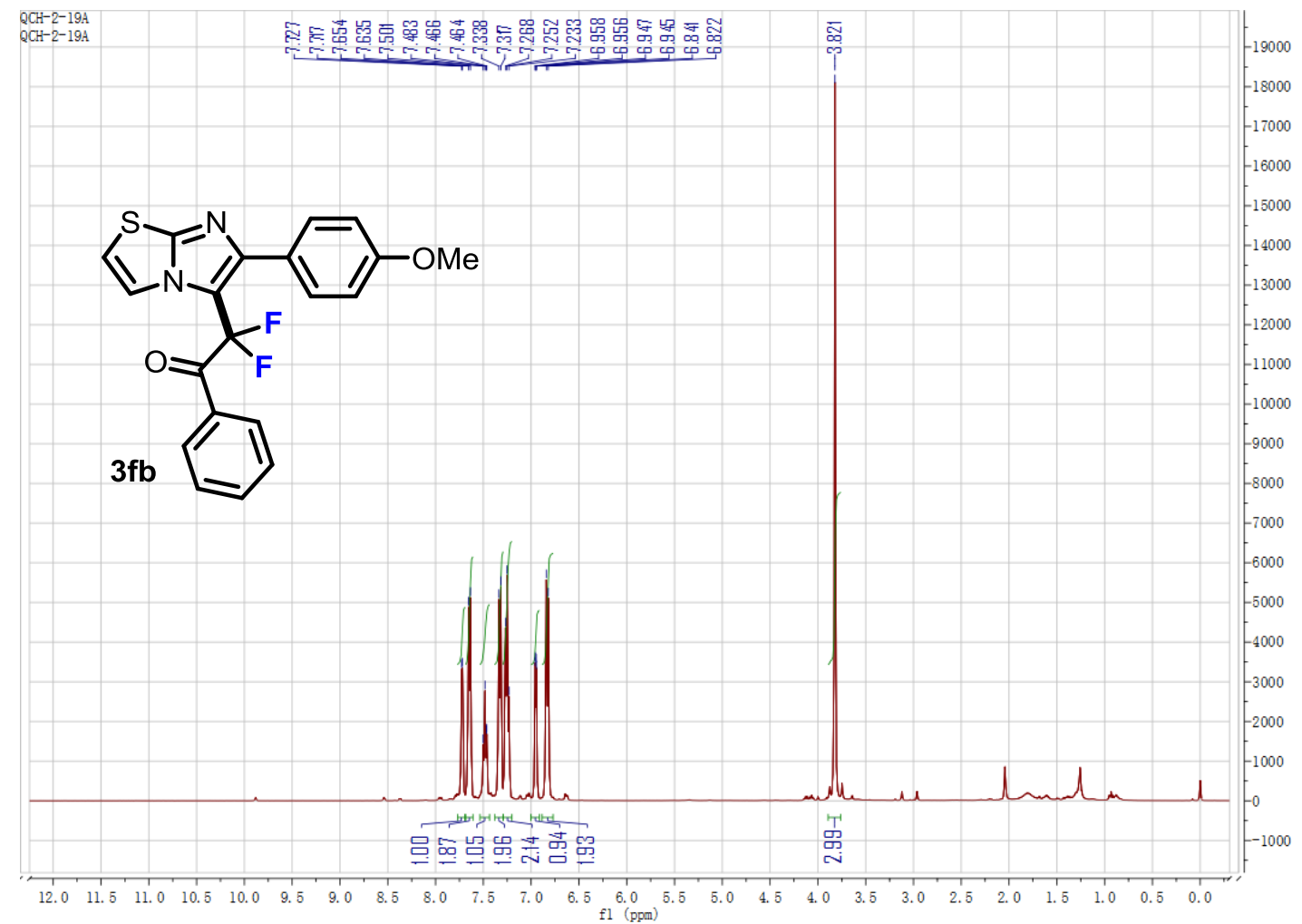

${ }^{19}$ F NMR (376 MHz, CDCl3): 


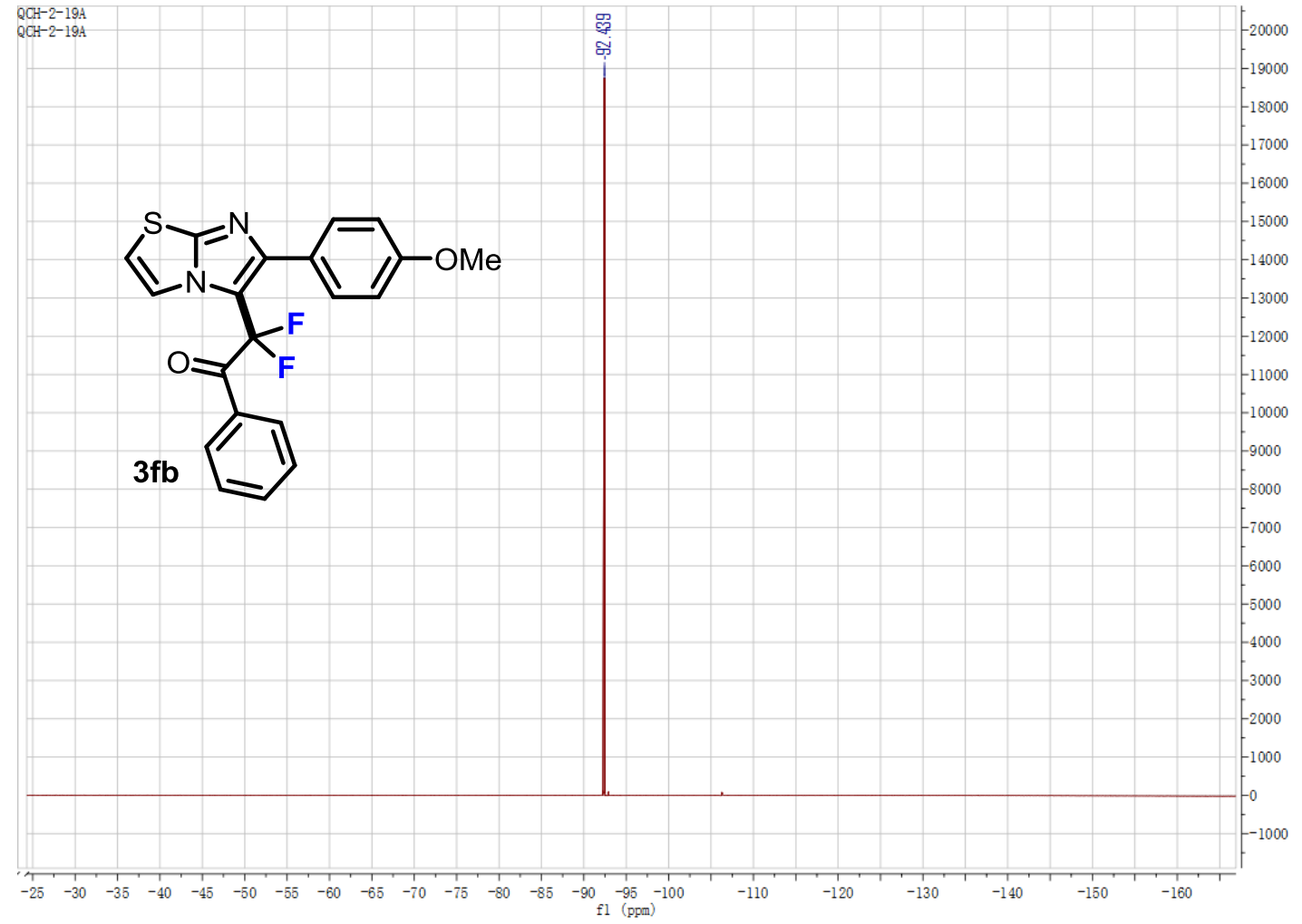

${ }^{13} \mathrm{C}$ NMR (100 MHz, $\left.\mathrm{CDCl}_{3}\right)$ :

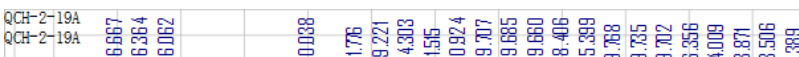

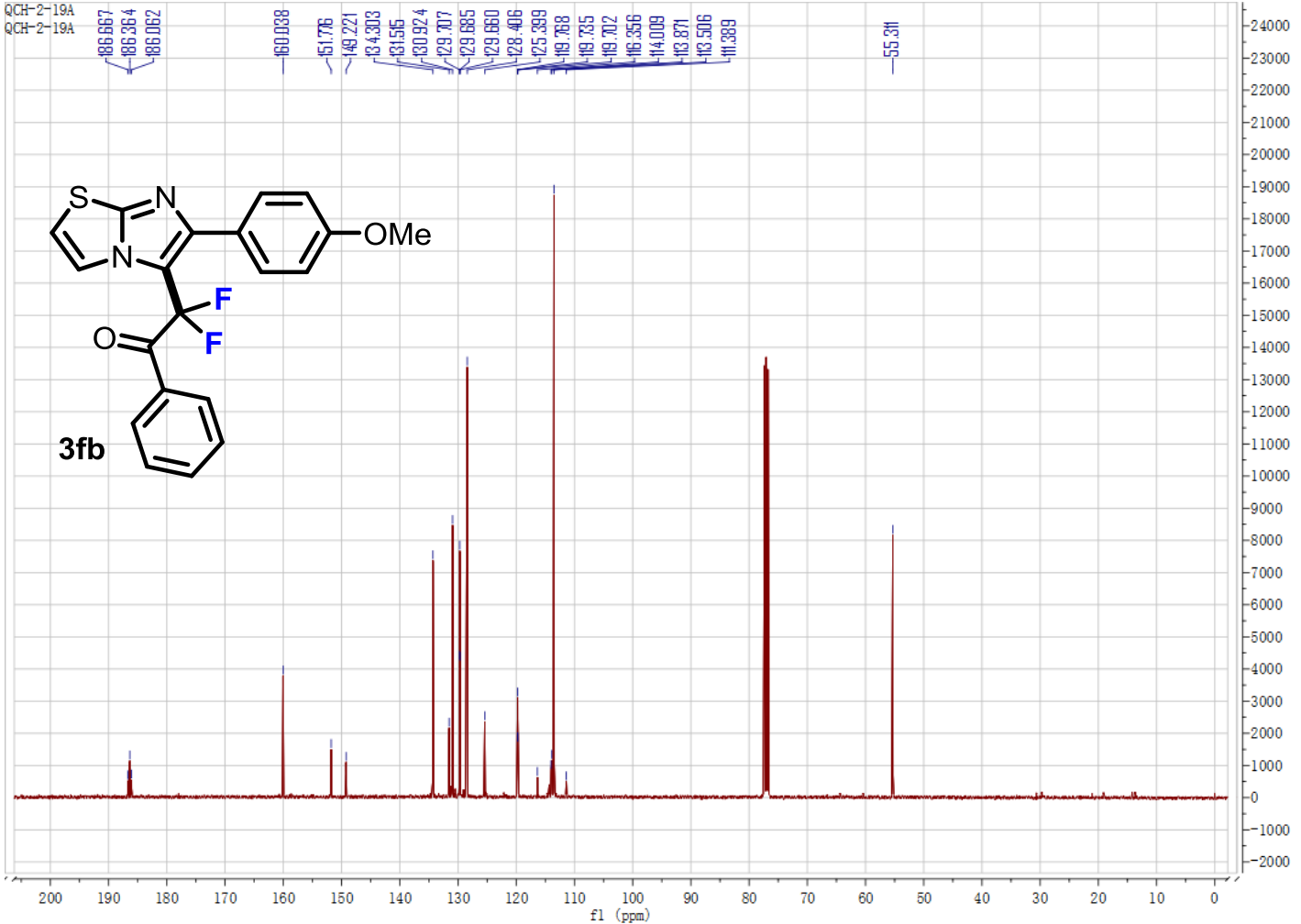


4-(5-(1,1-difluoro-2-oxo-2-phenylethyl)imidazo[2,1-b]thiazol-6-yl)benzonitrile (3fc) ${ }^{1}$ H NMR (400 MHz, CDCl3):

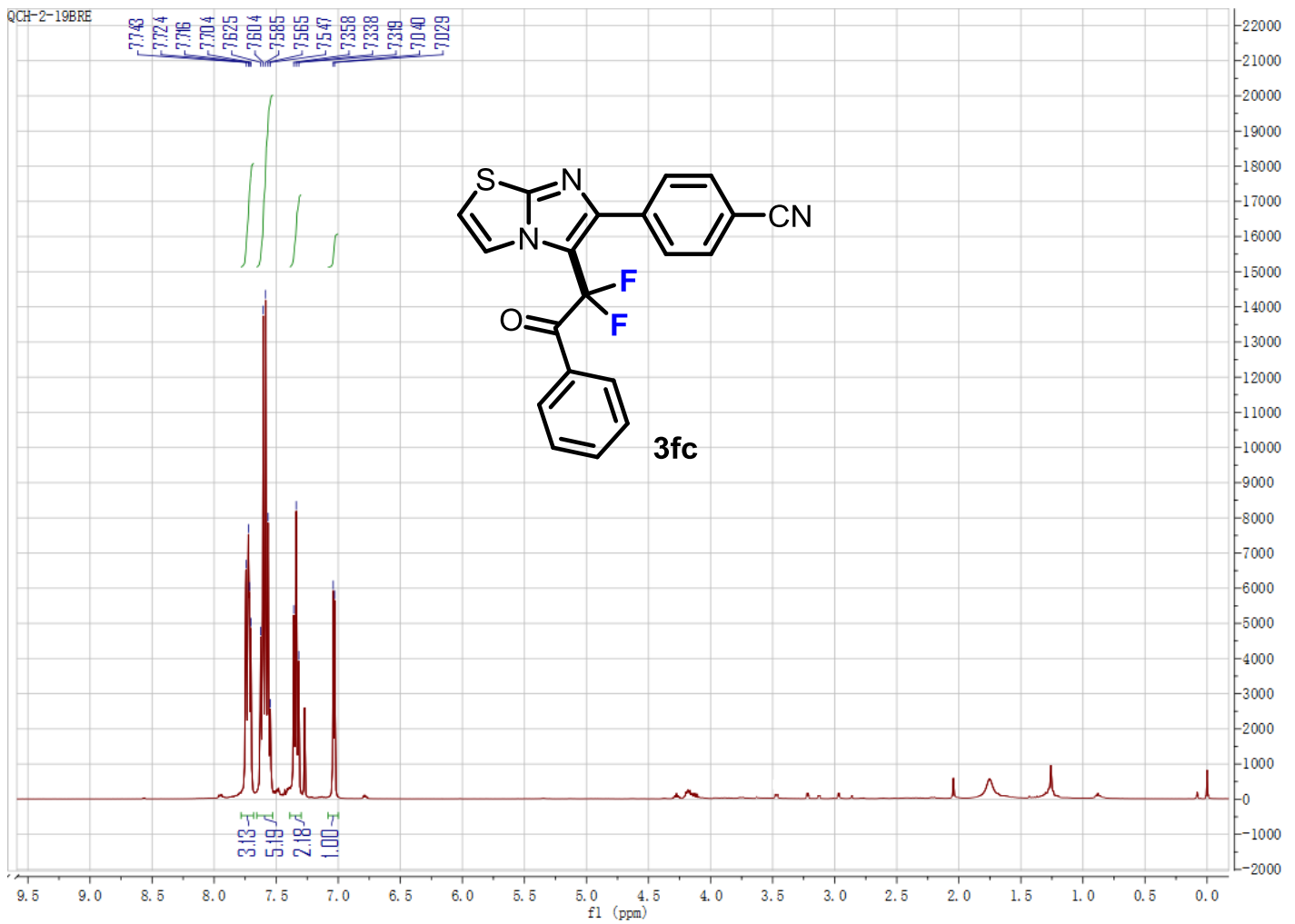

${ }^{19}$ F NMR(376 MHz, CDCl3): 


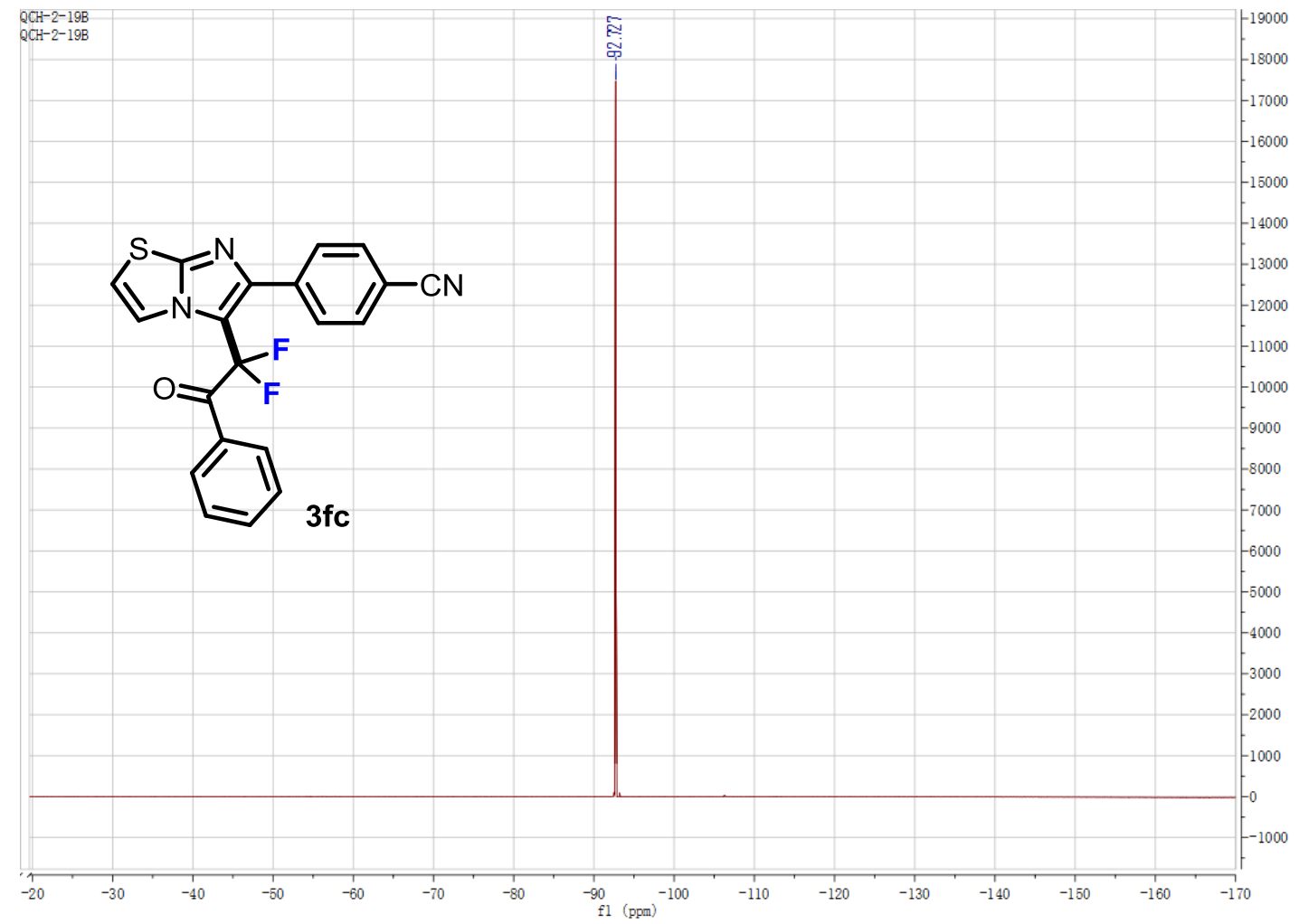

${ }^{13} \mathrm{C}$ NMR (100 MHz, $\left.\mathrm{CDCl}_{3}\right)$ :

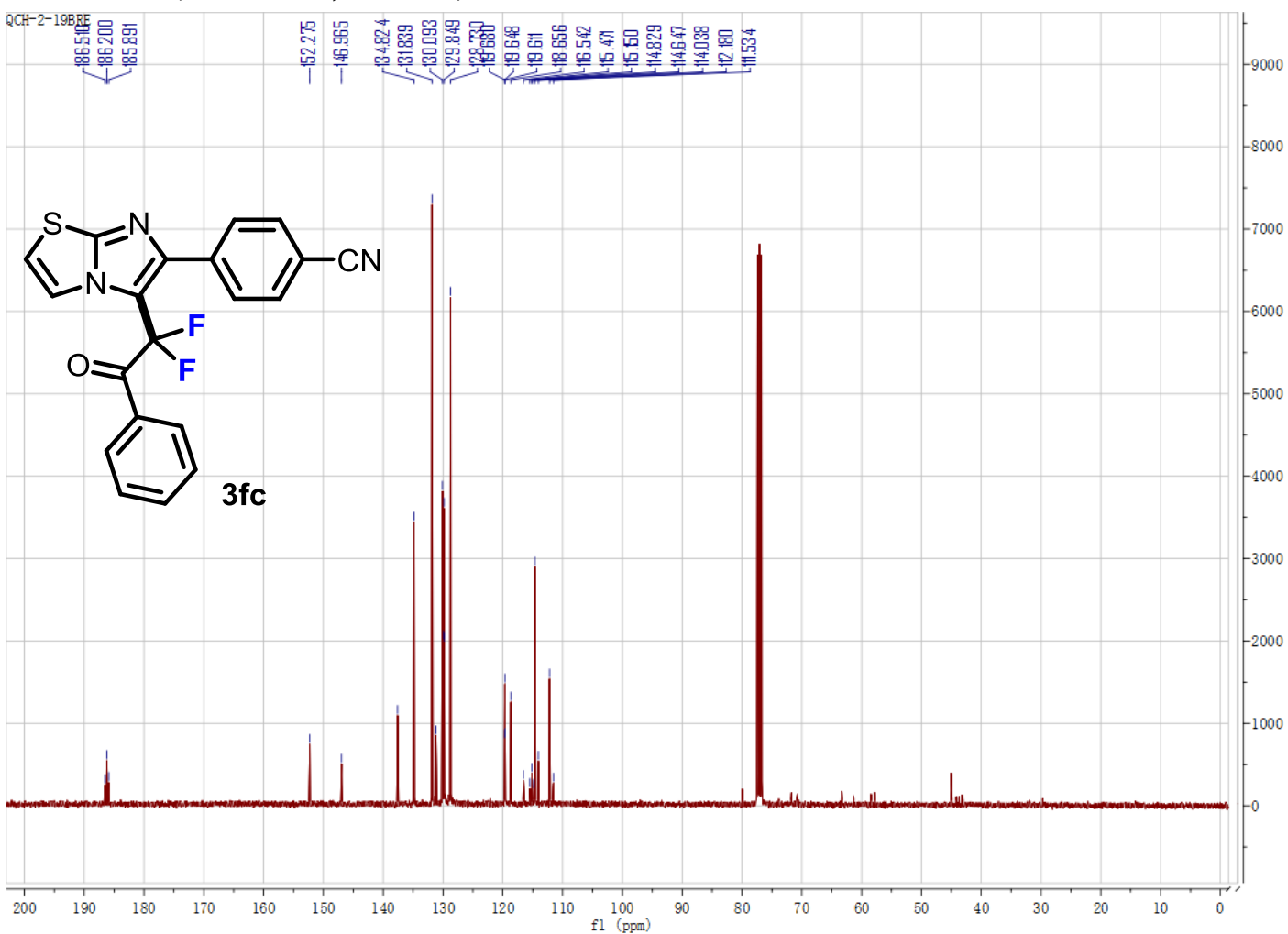


2-(6-(4-chlorophenyl)imidazo[2,1-b]thiazol-5-yl)-2,2-difluoro-1-phenylethan-1one (3fd)

${ }^{1} \mathrm{H}$ NMR (400 MHz, $\left.\mathrm{CDCl}_{3}\right)$ :

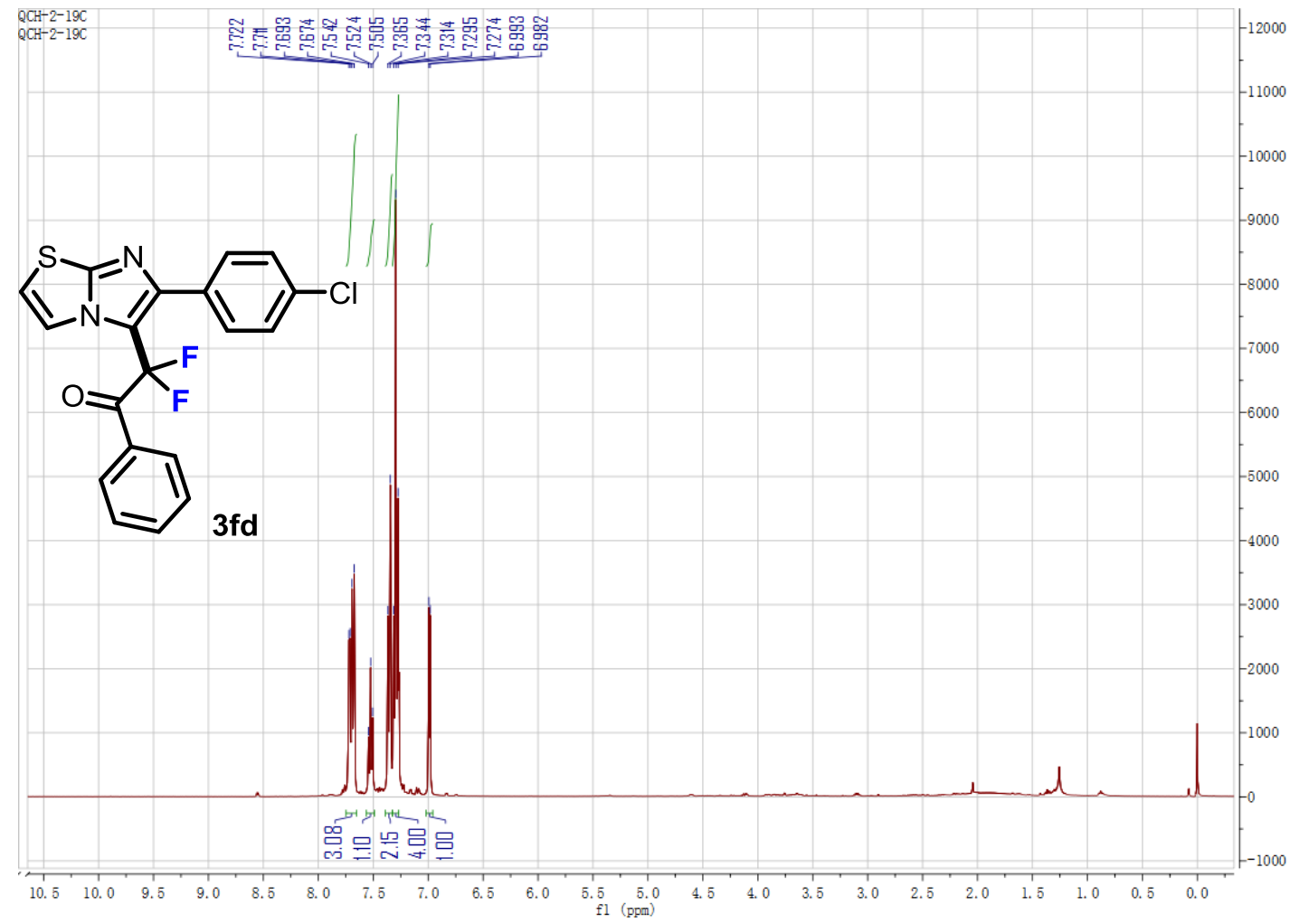


${ }^{19}$ F NMR (376 MHz, CDCl3):

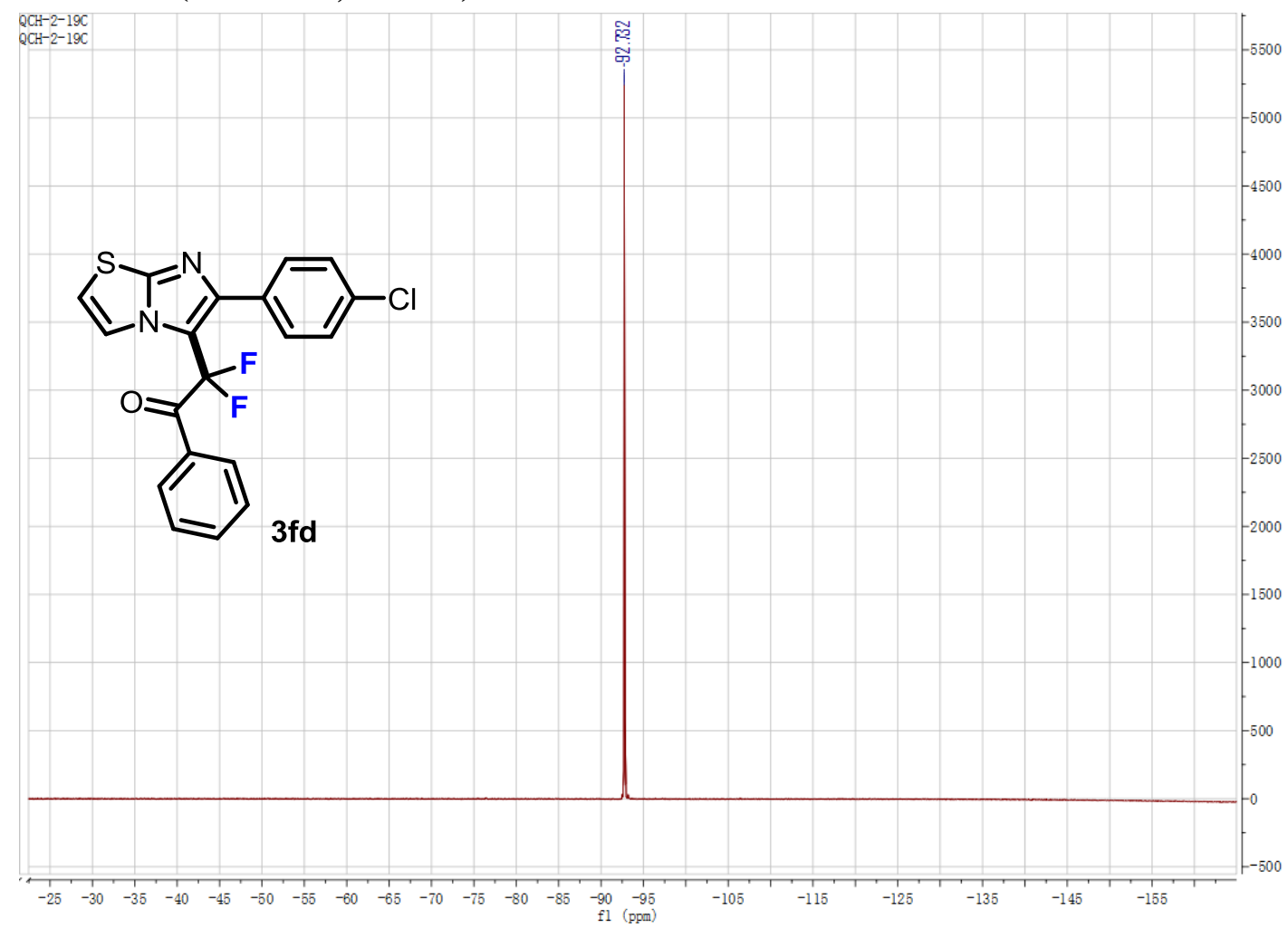

${ }^{13} \mathrm{C}$ NMR (100 MHz, CDCl 3$)$ :

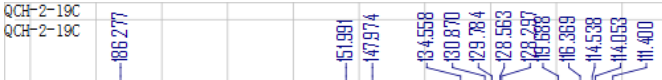

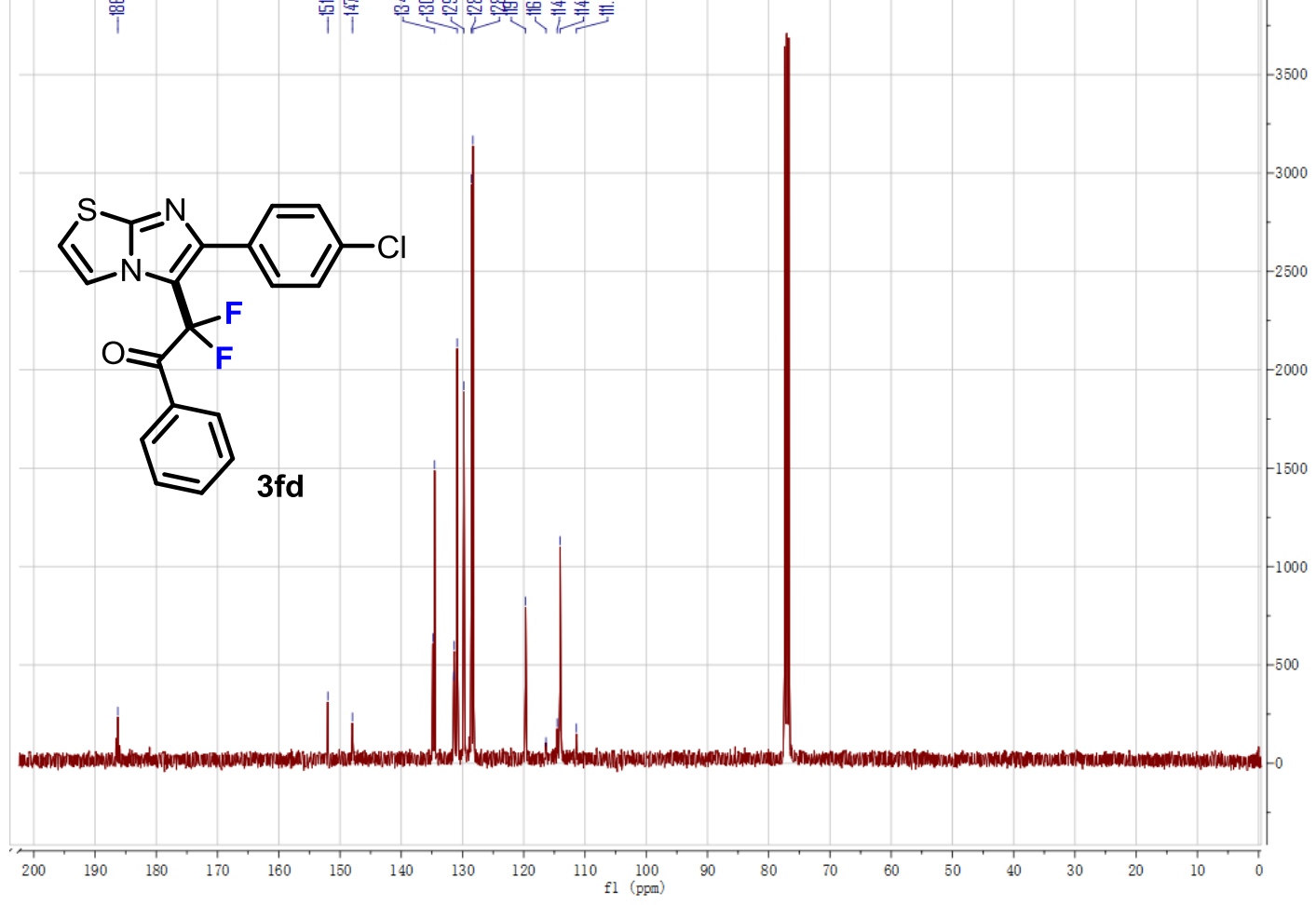


2,2-difluoro-1-phenyl-2-(2-phenylbenzo[d]imidazo[2,1-b]thiazol-3-yl)ethan-1-one (3ga) ${ }^{1} \mathrm{H}$ NMR (400 MHz, $\left.\mathrm{CDCl}_{3}\right)$ :

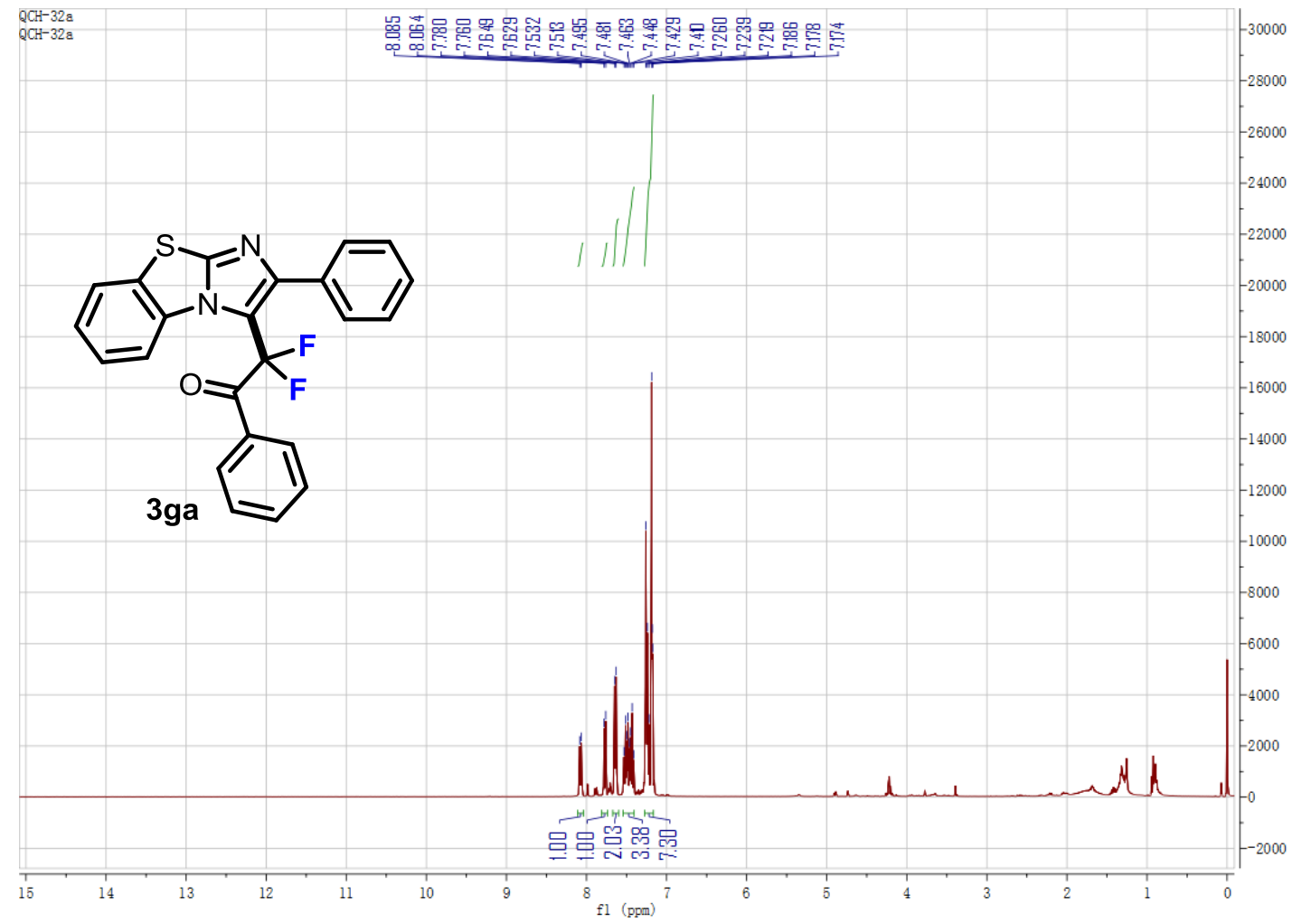


${ }^{19}$ F NMR (376 MHz, CDCl3):

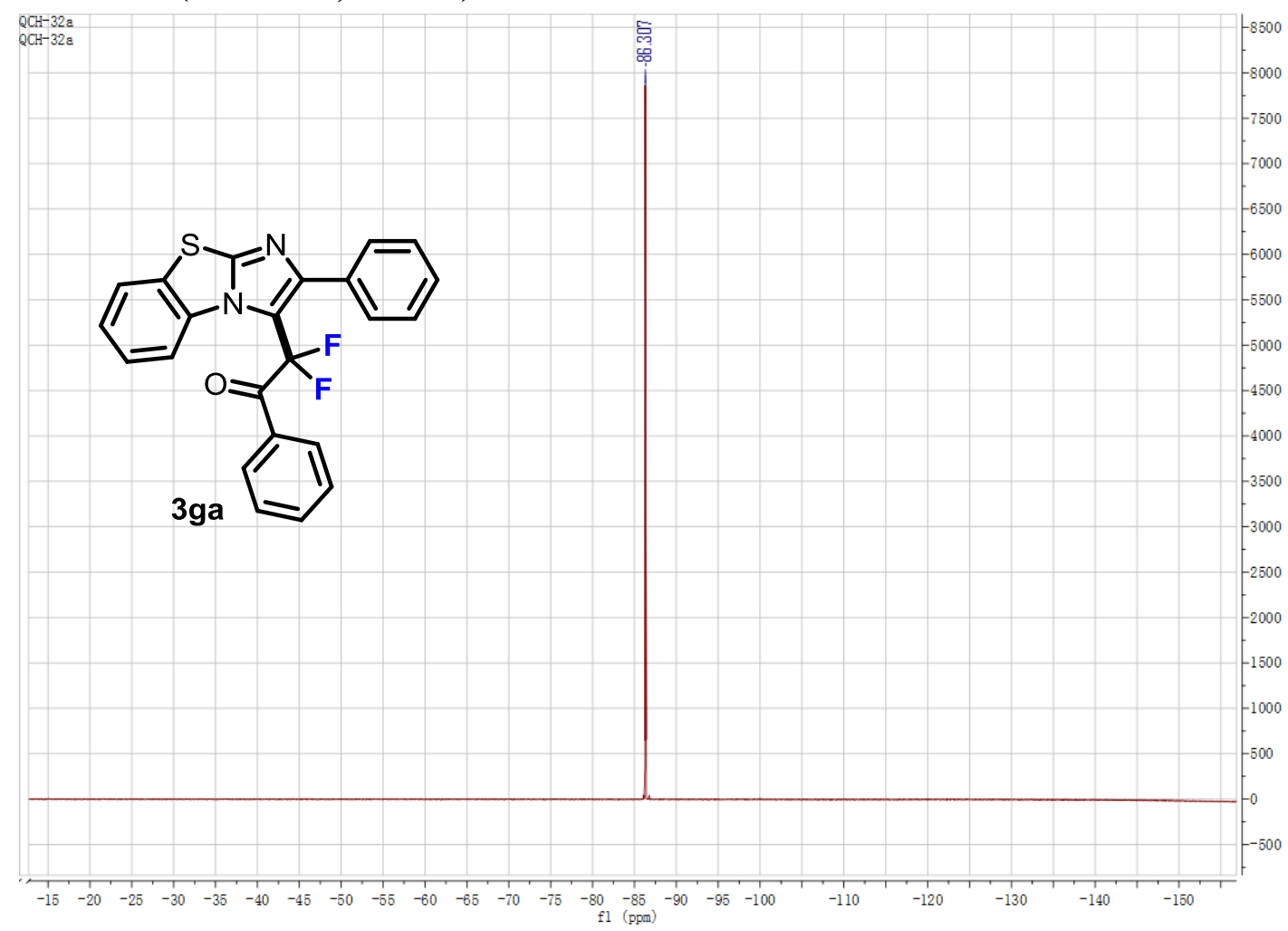

${ }^{13} \mathrm{C}$ NMR (100 MHz, CDCl 3$)$ :

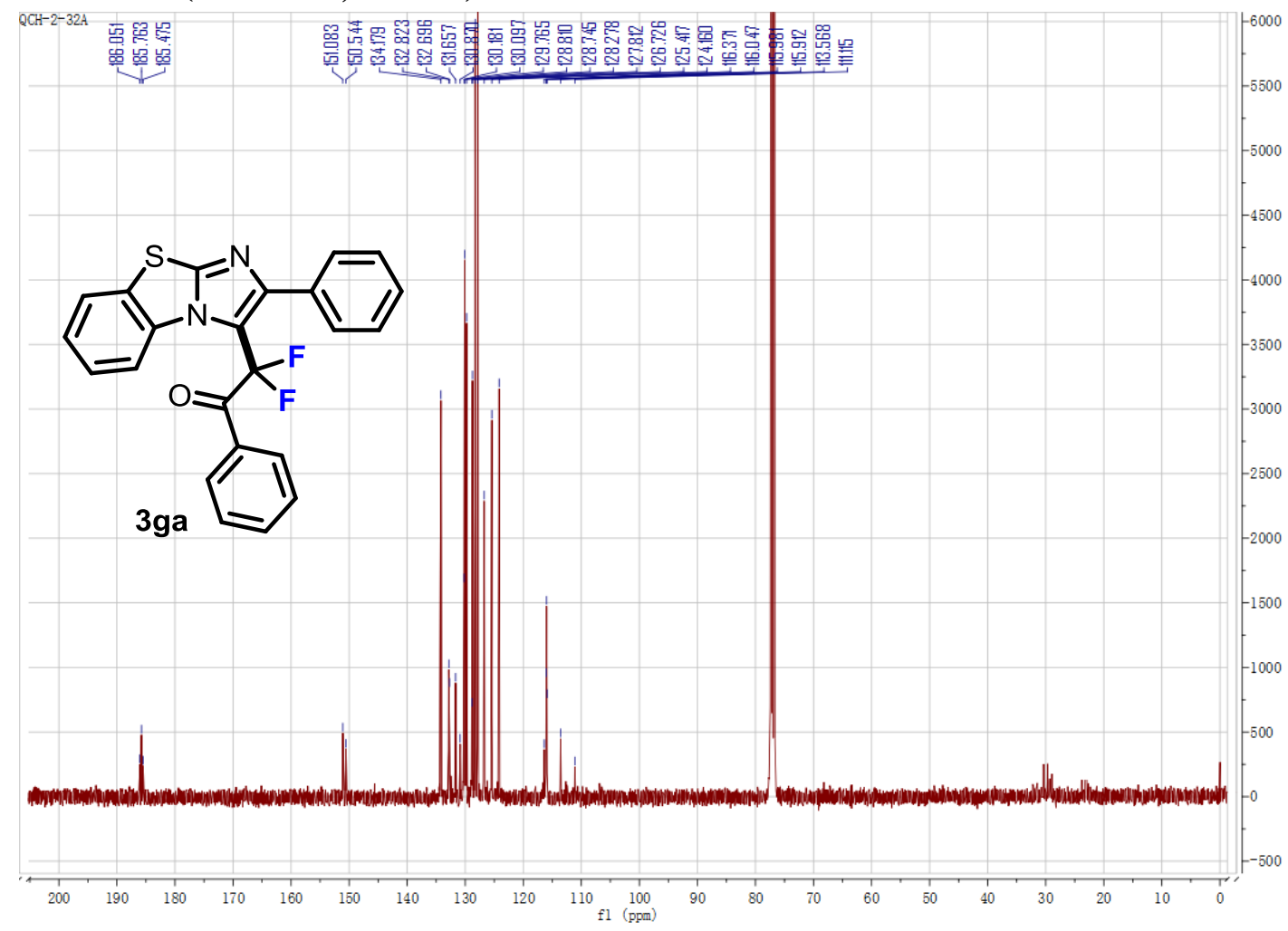


2,2-difluoro-2-(2-(4-methoxyphenyl)benzo[ $d]$ imidazo[2,1-b]thiazol-3-yl)-1-phenylethan1-one (3gb)

${ }^{1} \mathrm{H}$ NMR (400 MHz, $\mathrm{CDCl}_{3}$ ):

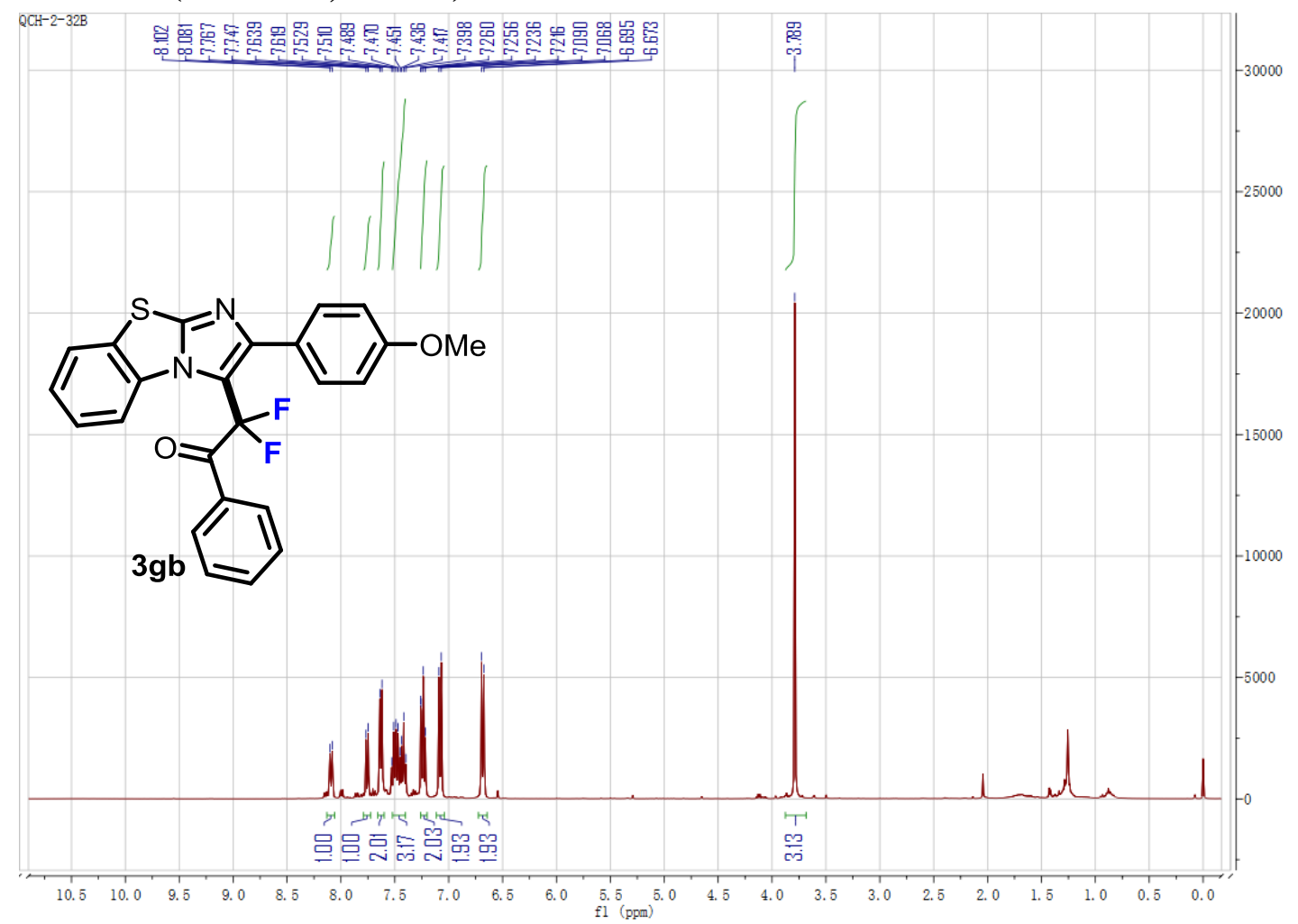


${ }^{19}$ F NMR (376 MHz, CDCl3):

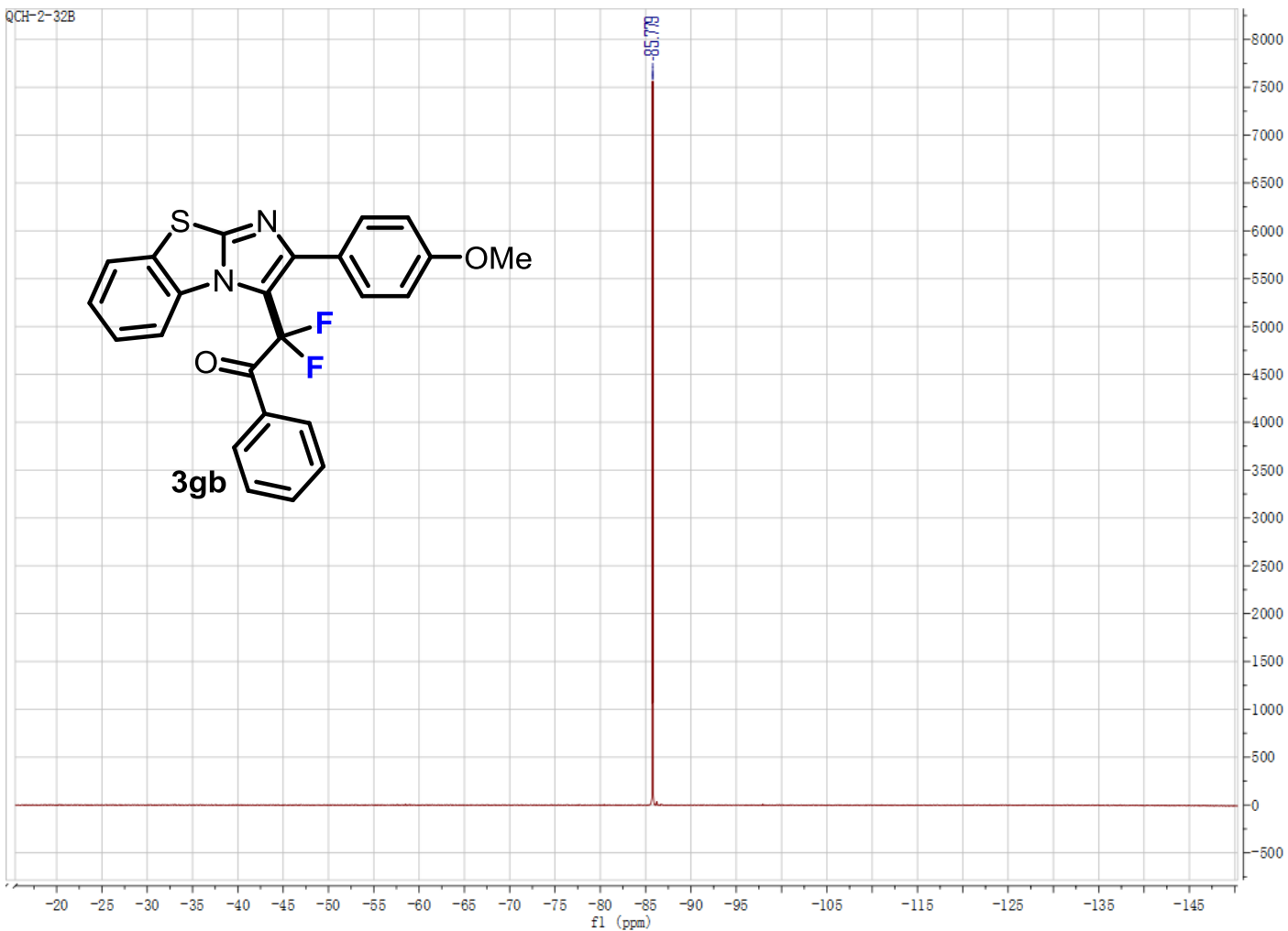

${ }^{13} \mathrm{C}$ NMR (100 MHz, CDCl 3$)$ :

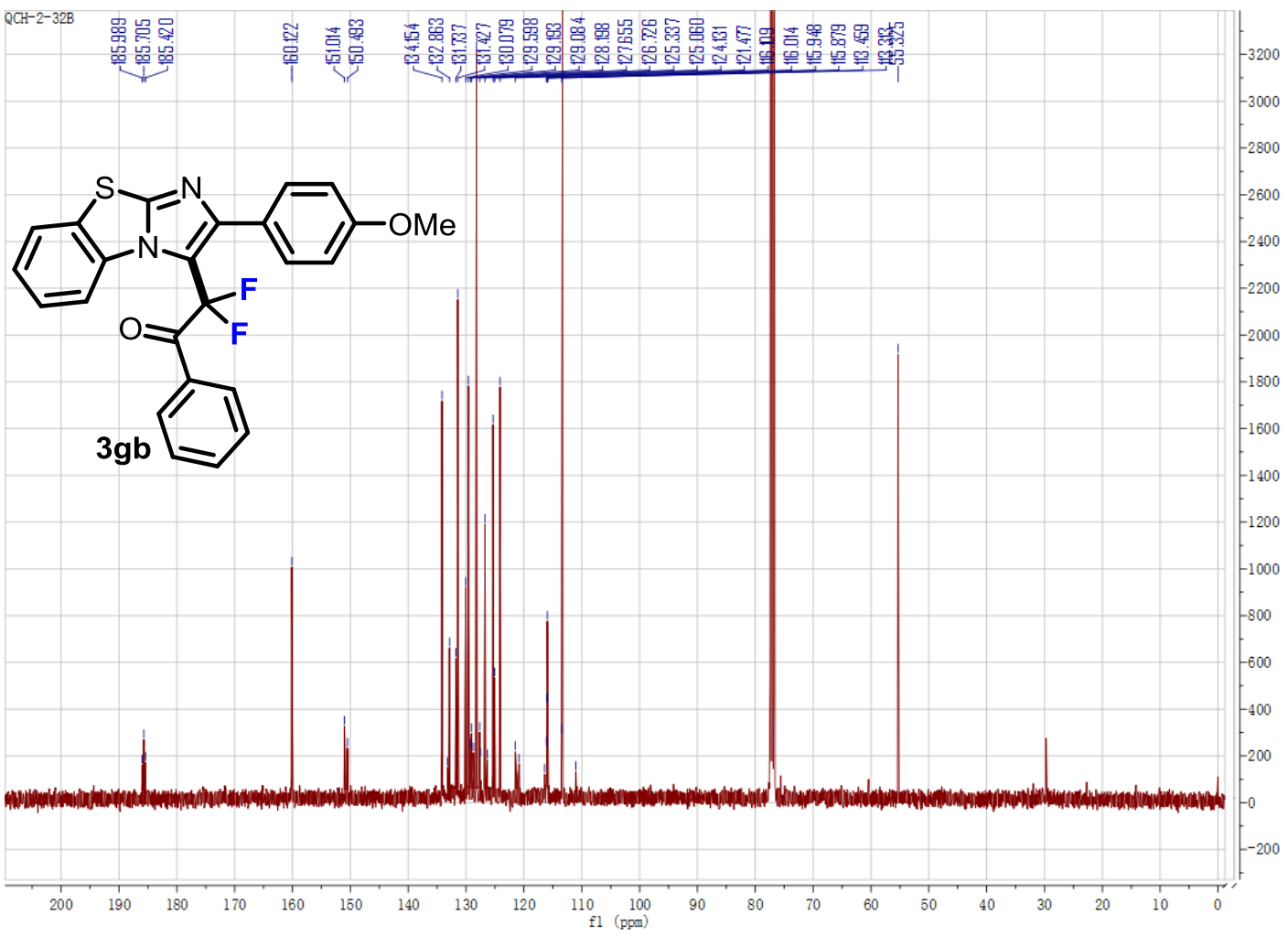


2-(2-(4-chlorophenyl)benzo[d]imidazo[2,1-b]thiazol-3-yl)-2,2-difluoro-1-phenylethan-1-o ne (3gc)

${ }^{1} \mathrm{H}$ NMR (400 MHz, $\left.\mathrm{CDCl}_{3}\right)$ :

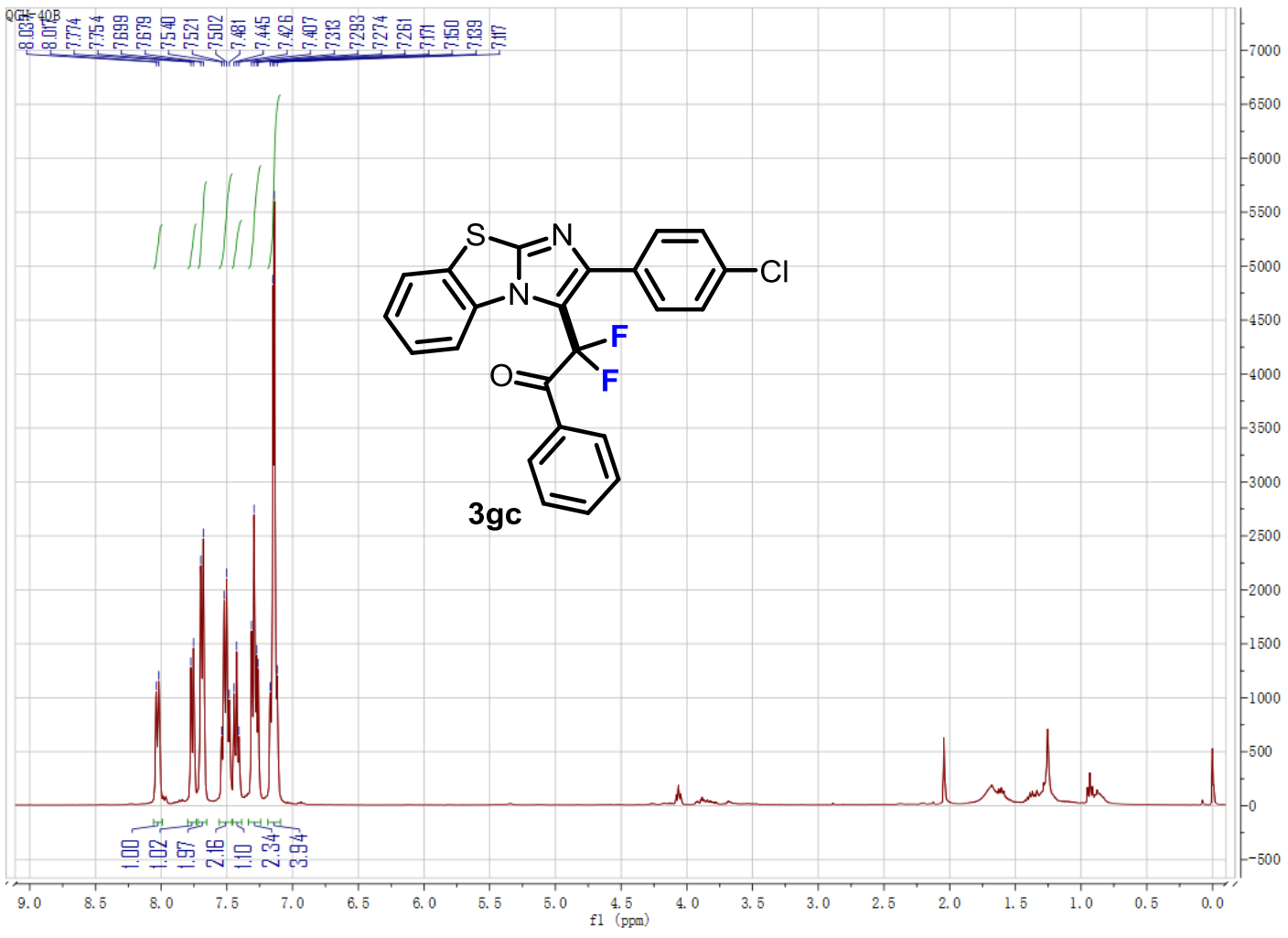


${ }^{19}$ F NMR (376 MHz, CDCl 3$)$ :

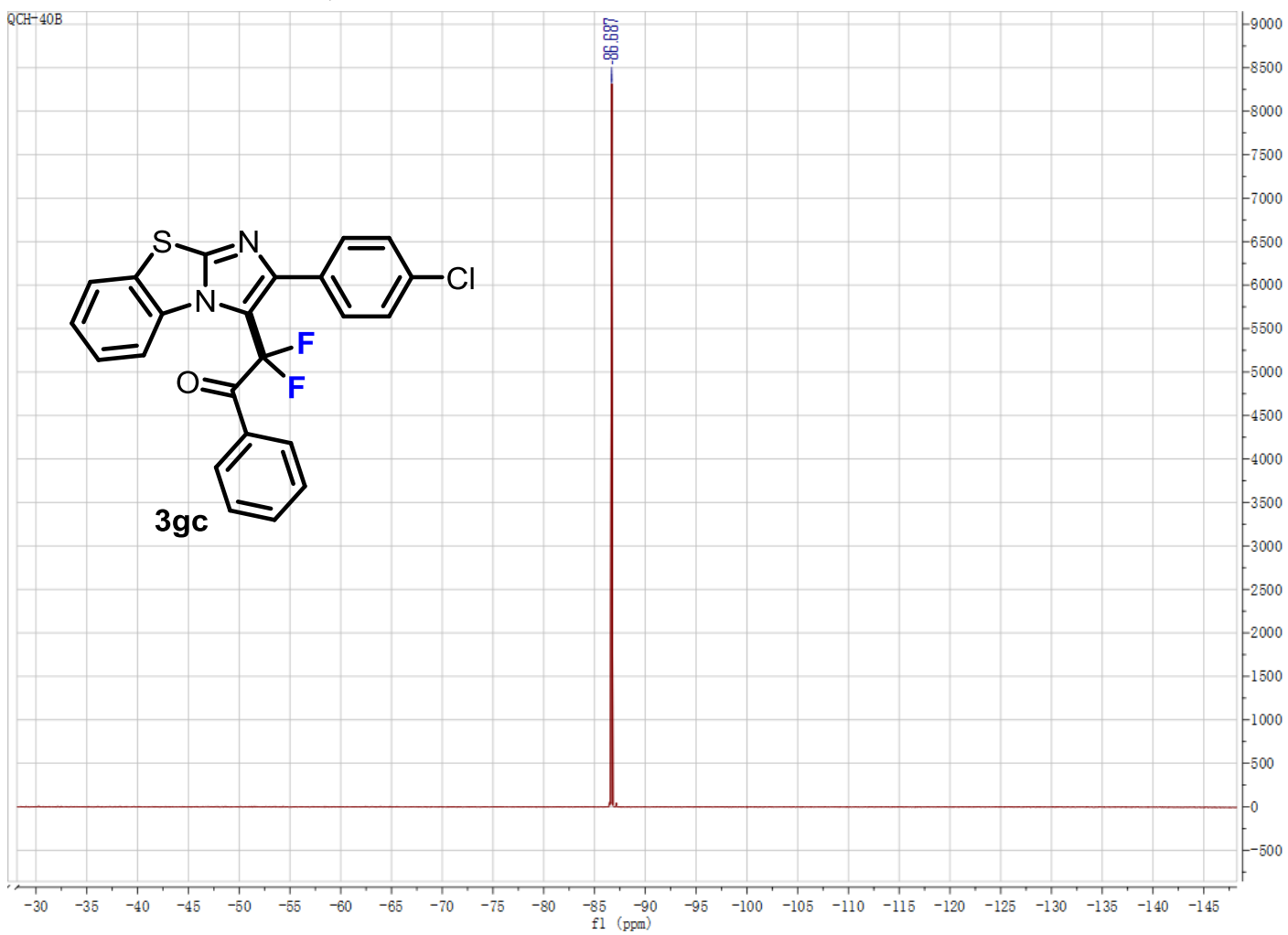

${ }^{13} \mathrm{C}$ NMR (100 MHz, CDCl 3$)$ :

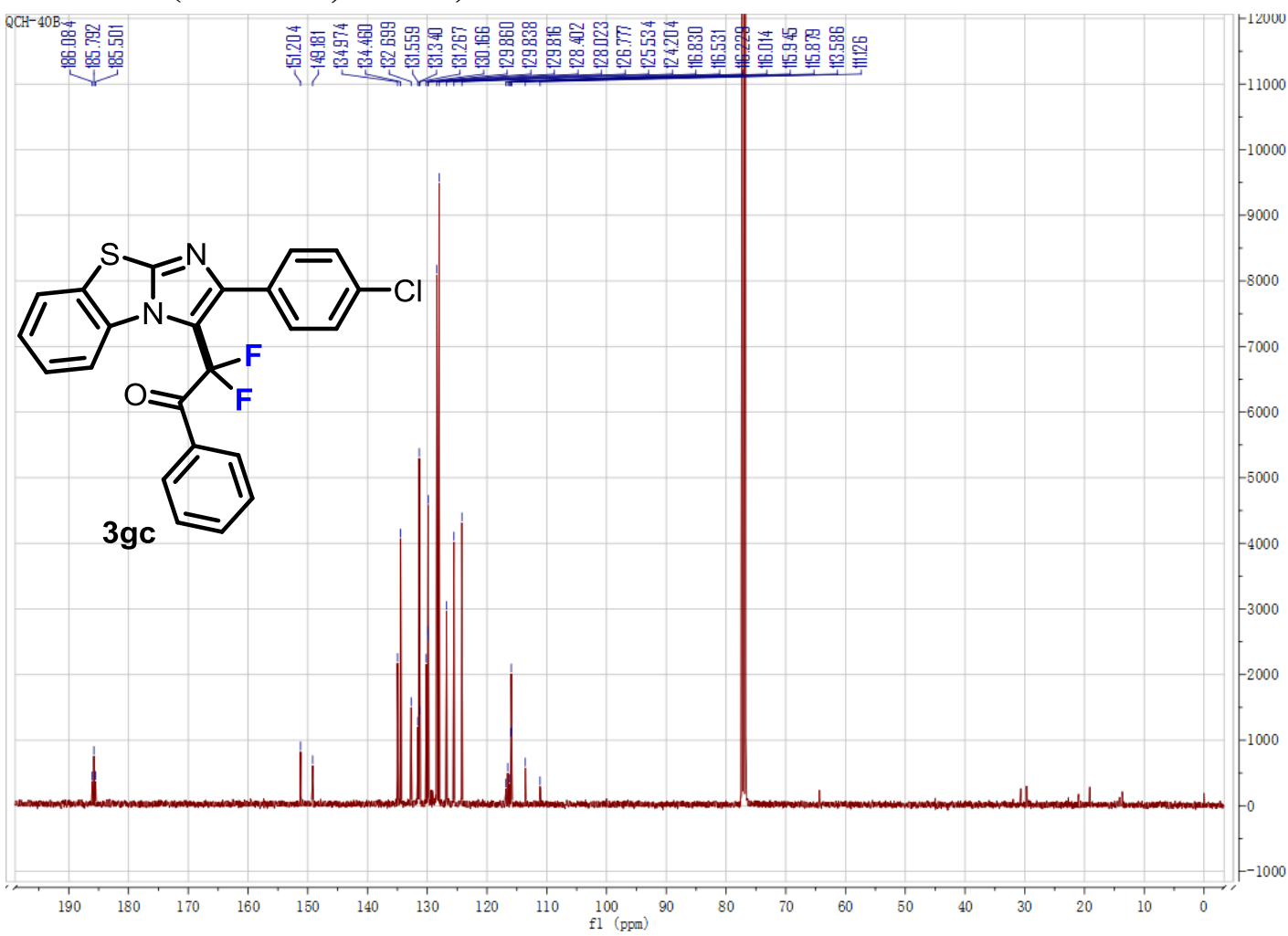


2,2-difluoro-2-(2-(2-fluorophenyl)benzo[ $[d]$ imidazo[2,1-b]thiazol-3-yl)-1-phenylethan-1-o ne (3gd)

${ }^{1} \mathrm{H}$ NMR (400 MHz, $\left.\mathrm{CDCl}_{3}\right)$ :

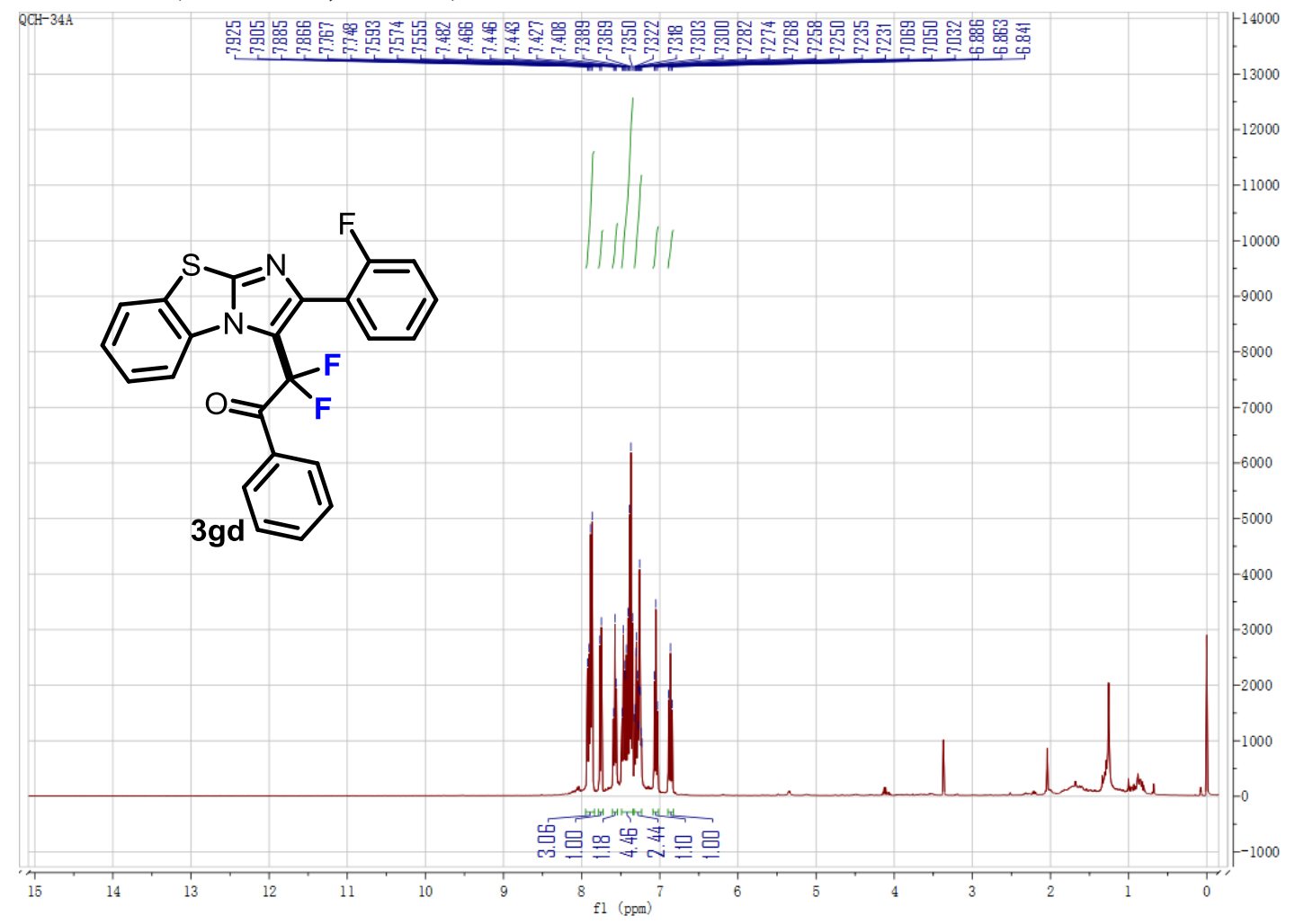


${ }^{19}$ F NMR (376 MHz, CDCl3):

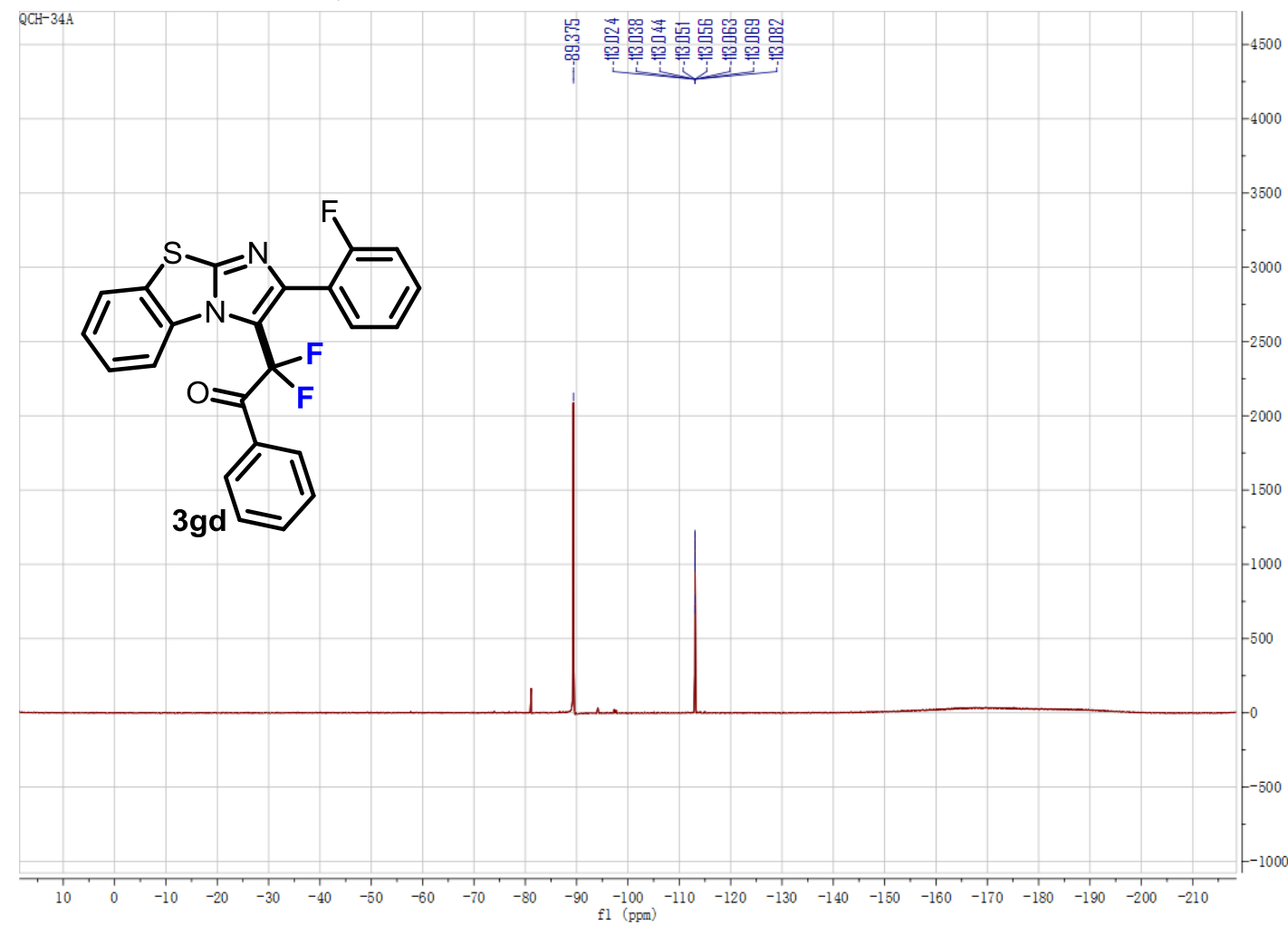

${ }^{13} \mathrm{C}$ NMR (100 MHz, CDCl 3$)$ :

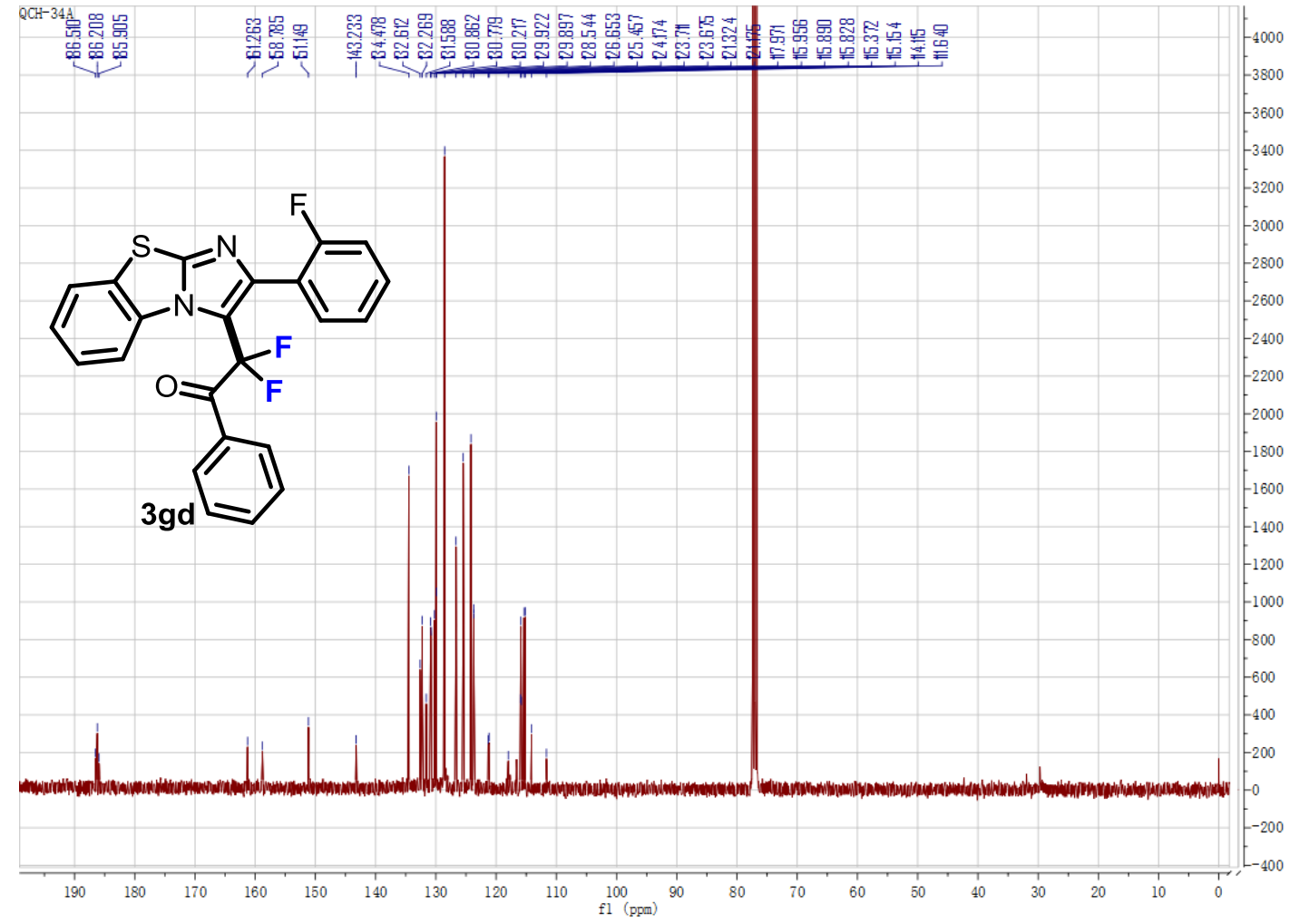


2,2-difluoro-1-phenyl-2-(2-phenylimidazo[1,2-a]pyridin-3-yl)ethan-1-one (3ha) ${ }^{1} \mathrm{H}$ NMR (400 MHz, CDCl $)$ :

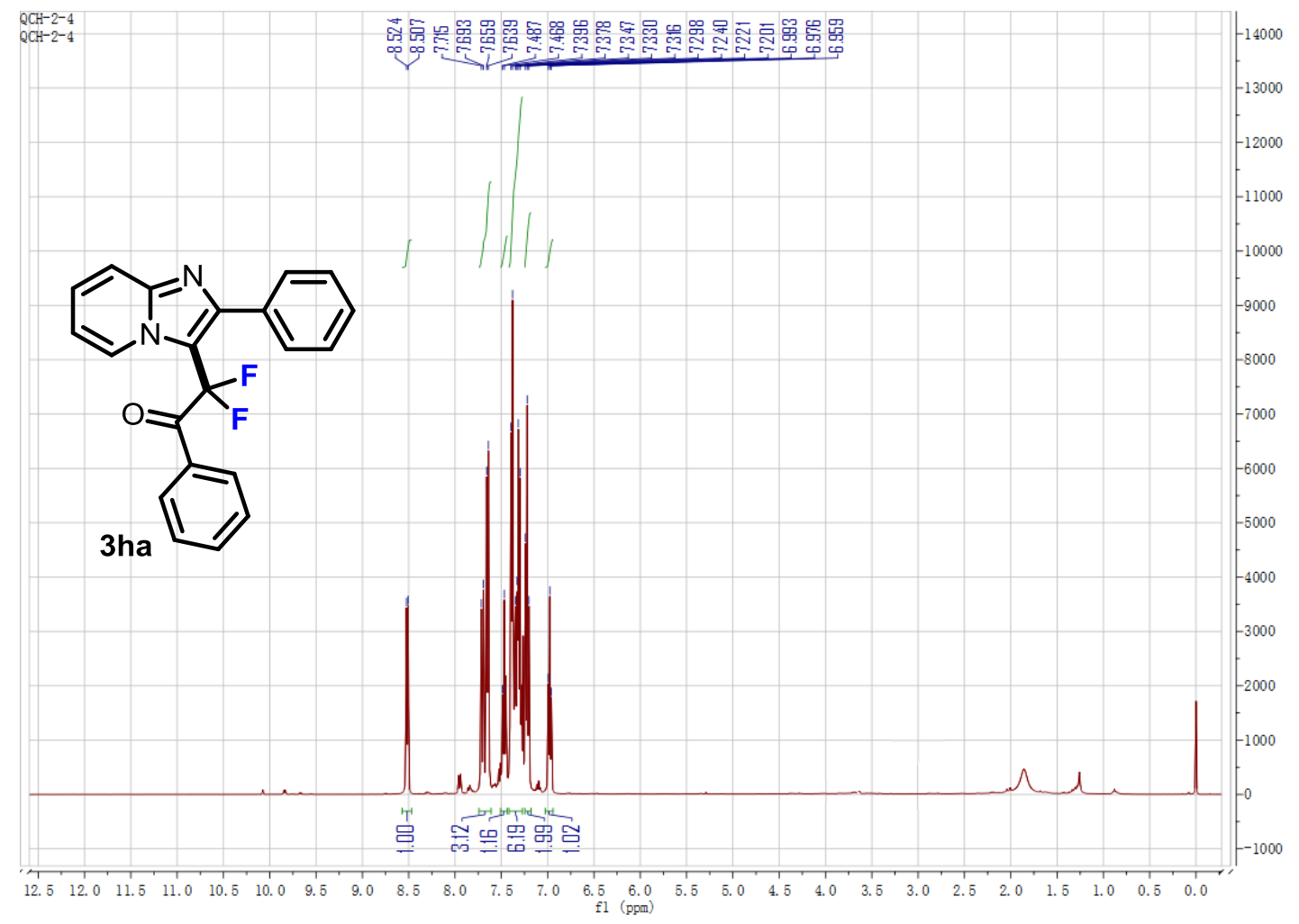


${ }^{19}$ F NMR (376 MHz, CDCl3):

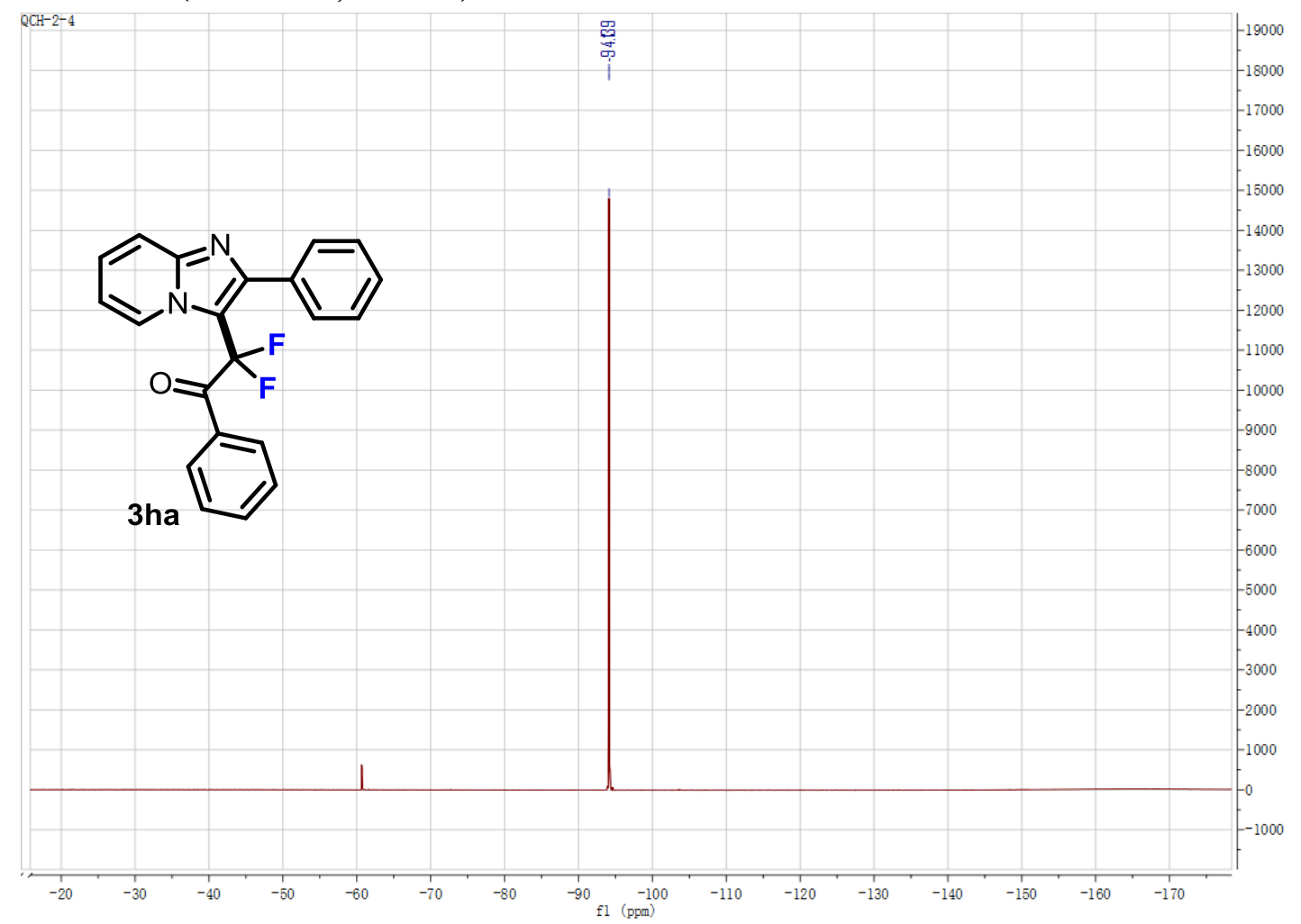

${ }^{13} \mathrm{C}$ NMR (100 MHz, CDCl3):

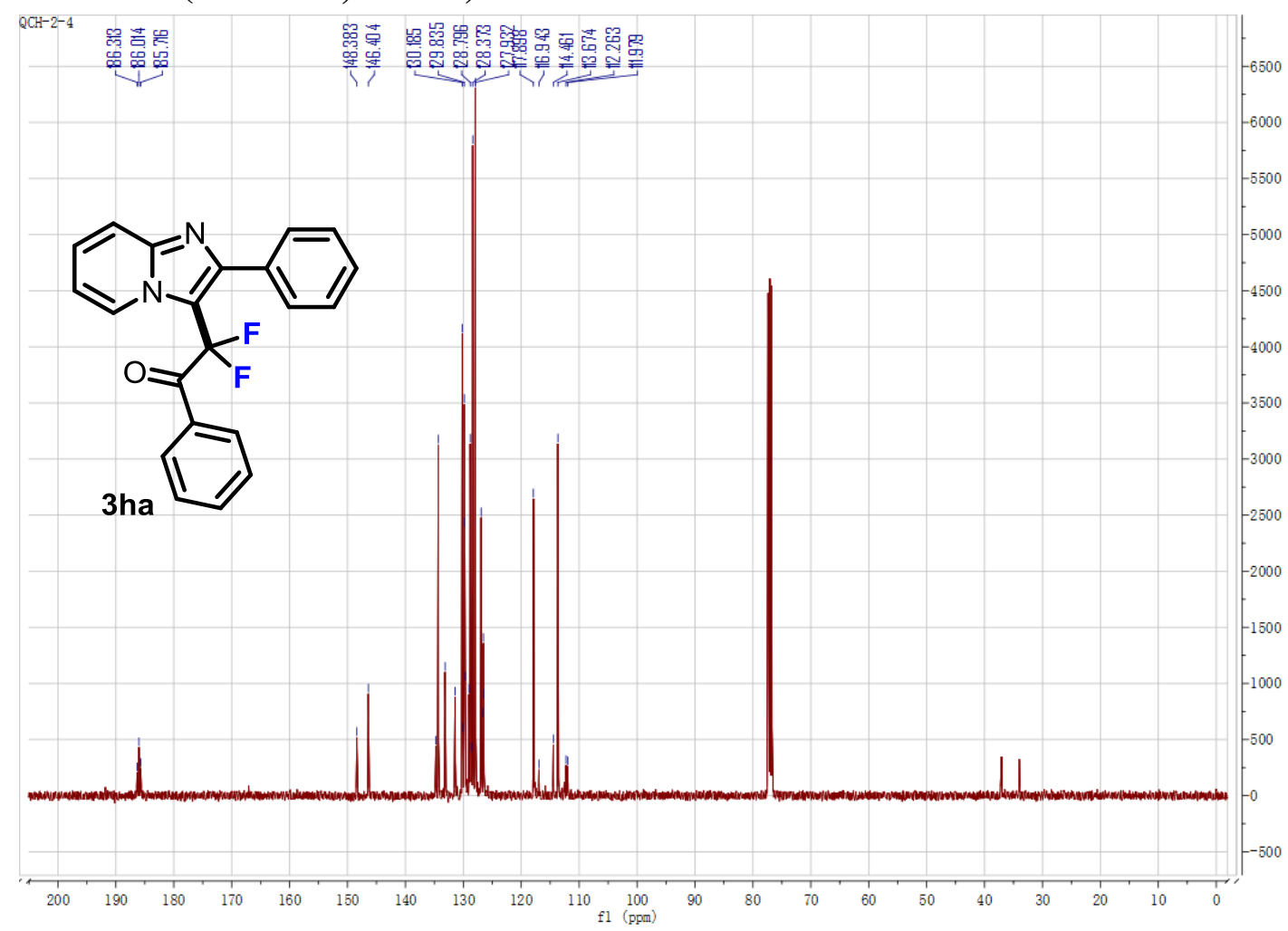


2,2-difluoro-2-(2-phenylimidazo[1,2-a]pyridin-3-yl)-1-(p-tolyl)ethan-1-one (3hb) ${ }^{1} \mathrm{H}$ NMR (400 MHz, CDCl 3$)$ :

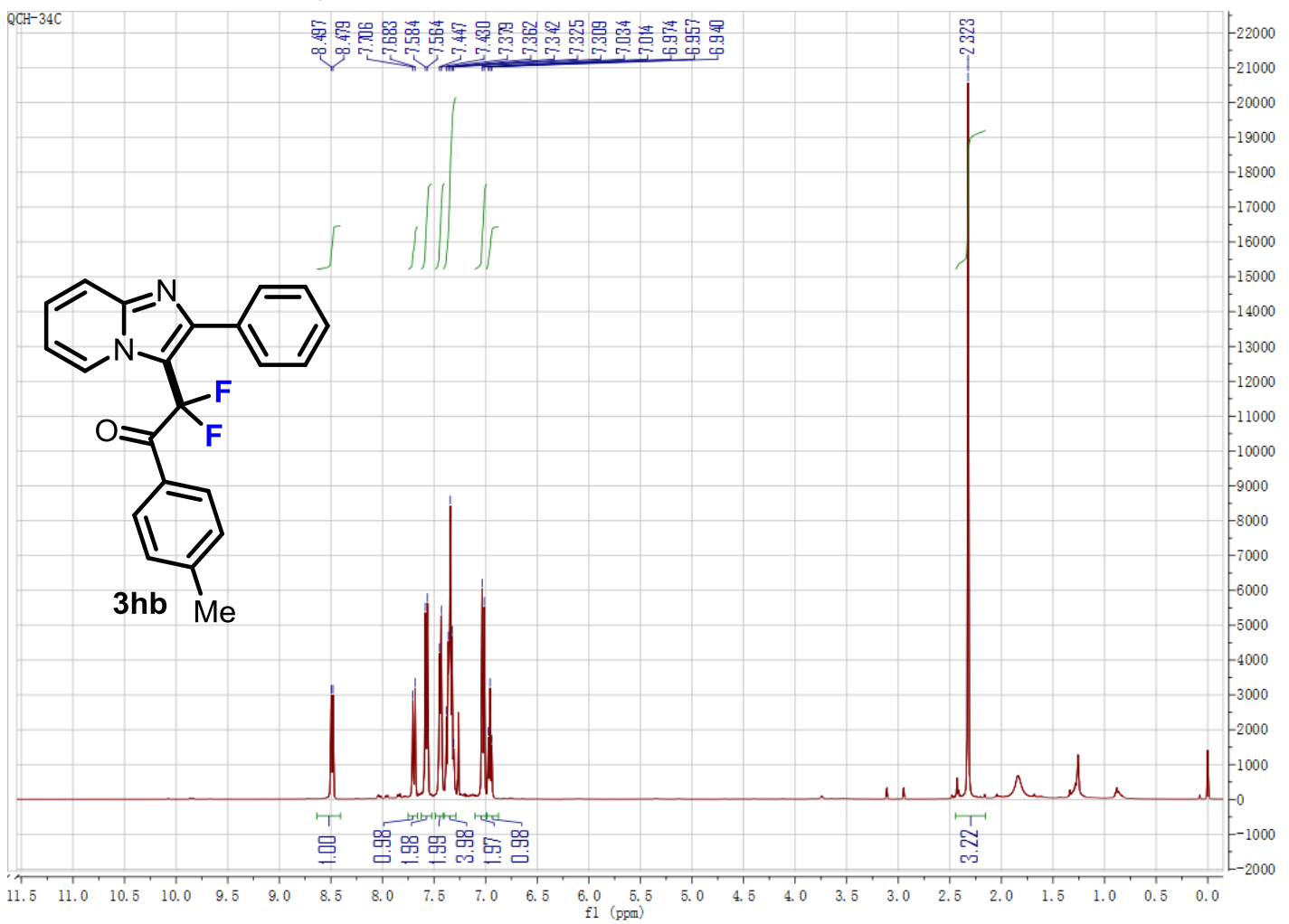


${ }^{19}$ F NMR (376 MHz, CDCl3):

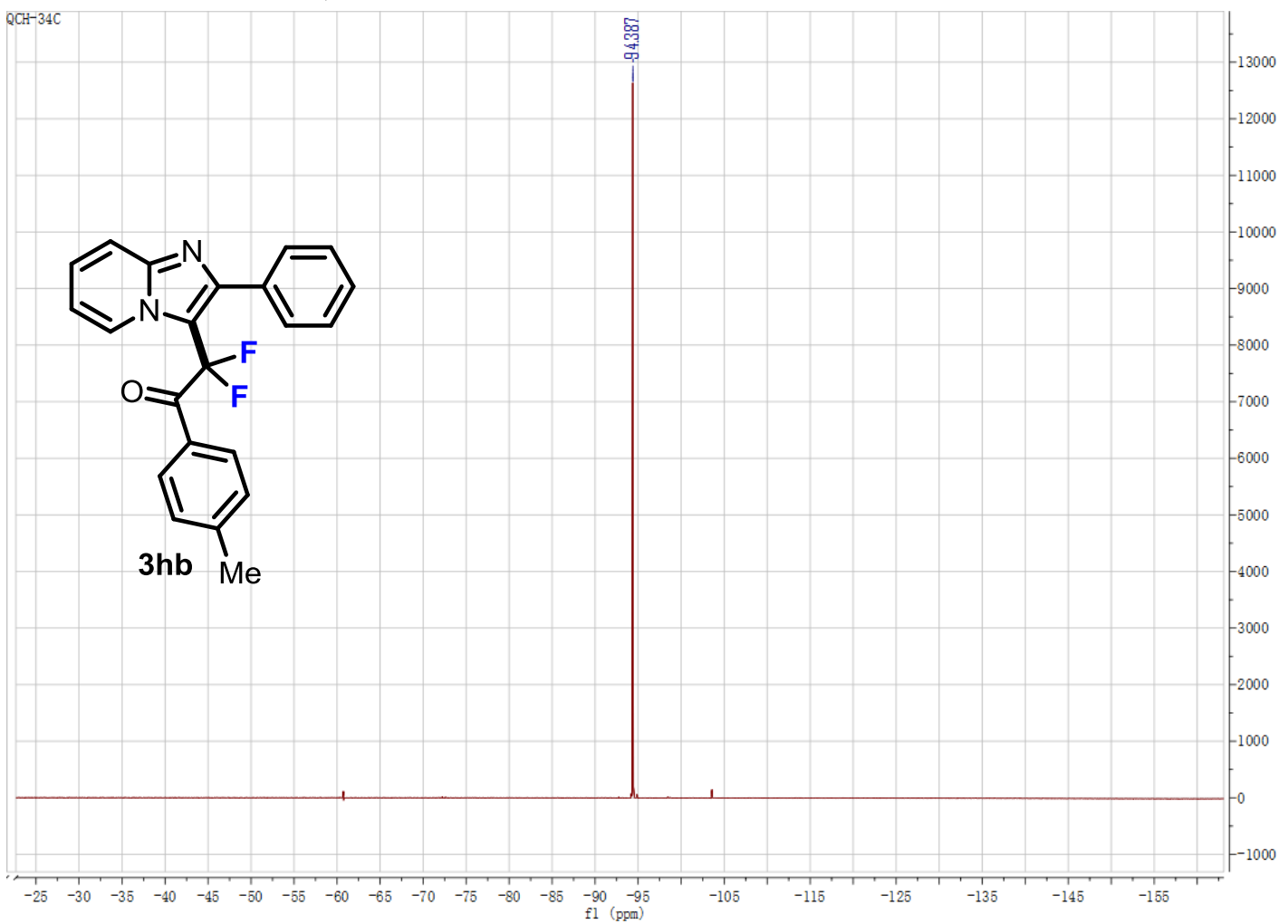

${ }^{13} \mathrm{C}$ NMR (100 MHz, CDCl3):

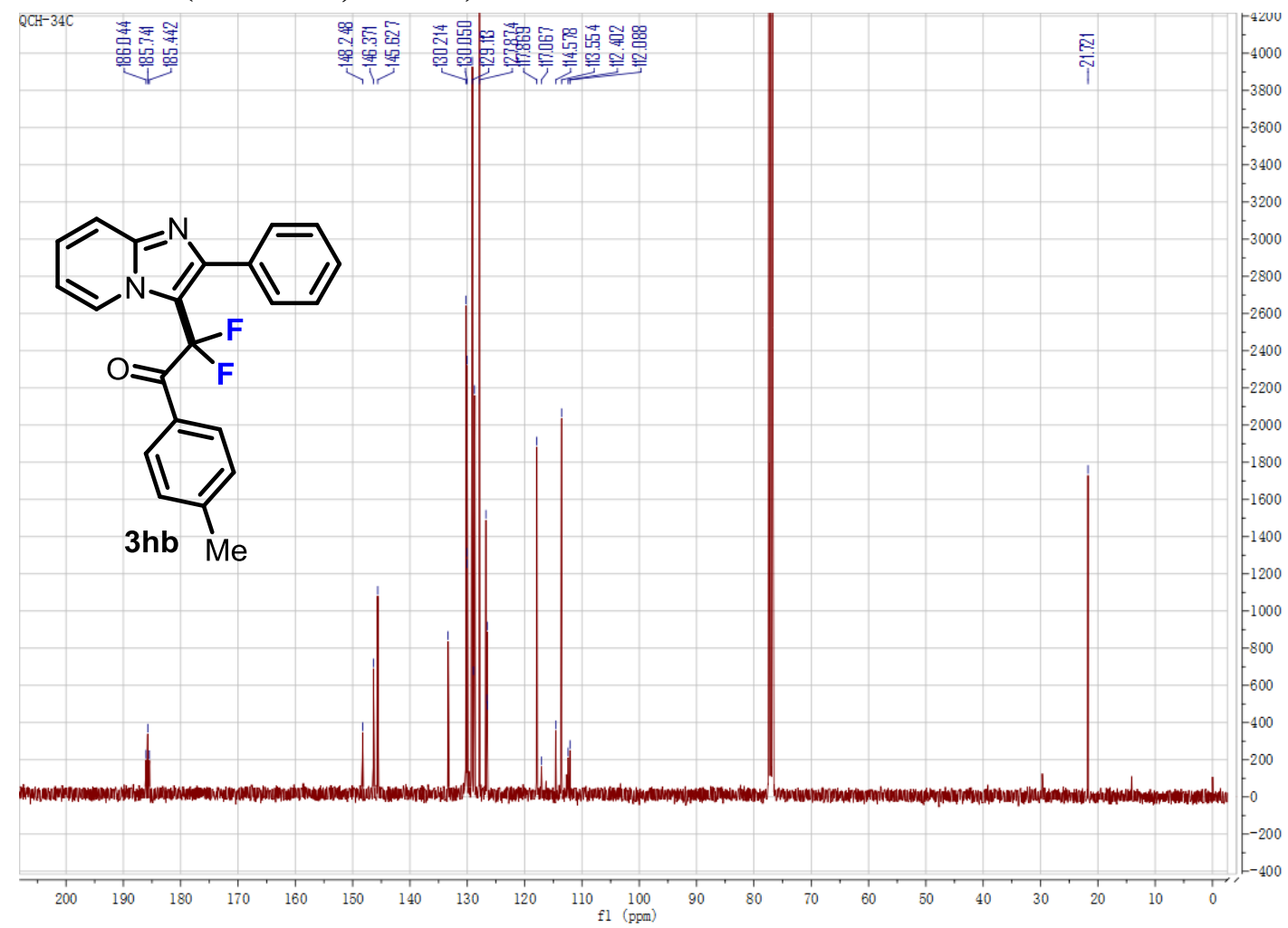


2,2-difluoro-1-(4-methoxyphenyl)-2-(2-phenylimidazo[1,2-a]pyridin-3-yl)ethan-1-one (3hc)

${ }^{1} \mathrm{H}$ NMR (400 MHz, CDCl3): 


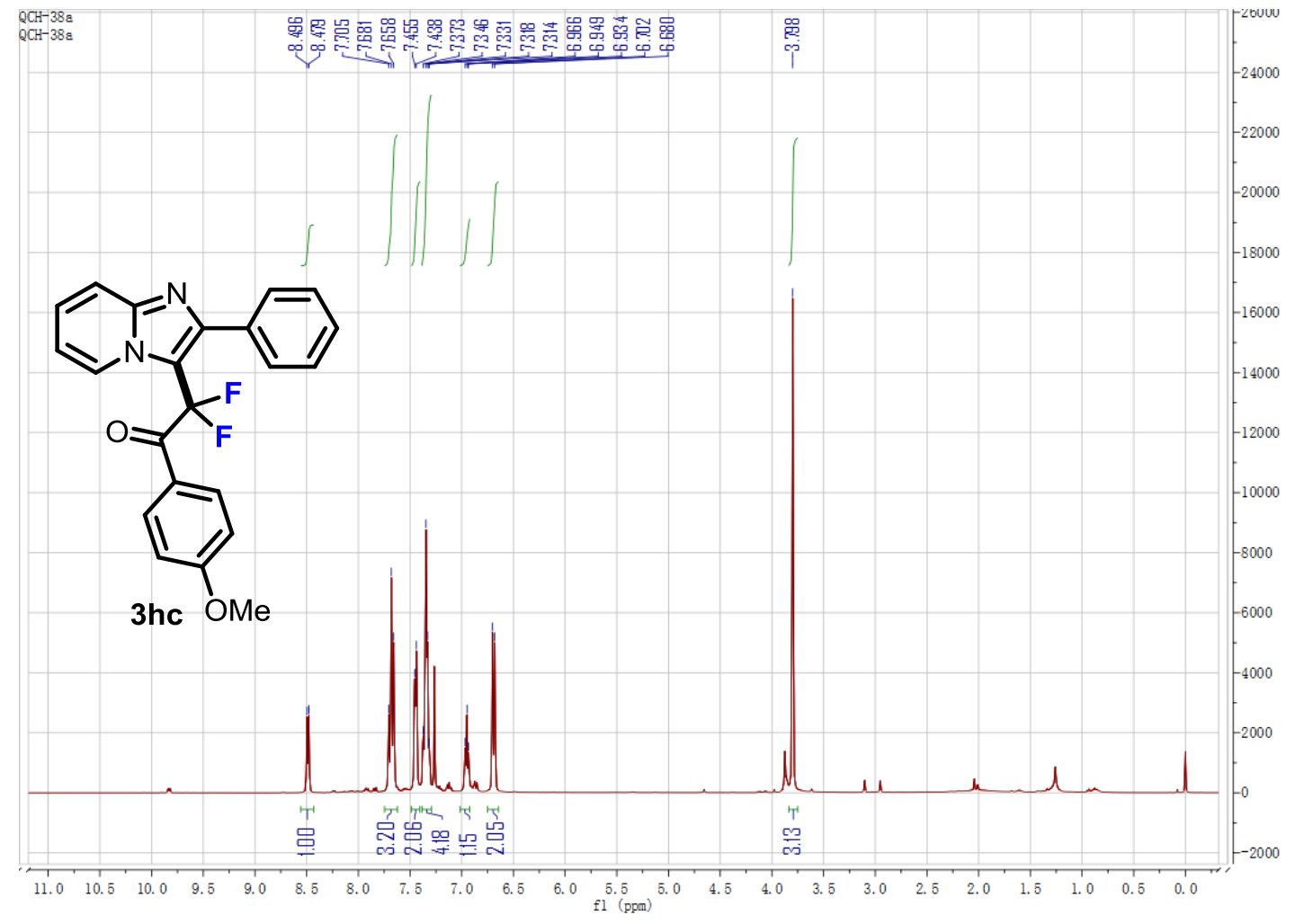

${ }^{19}$ F NMR (376 MHz, CDCl $)$ ):

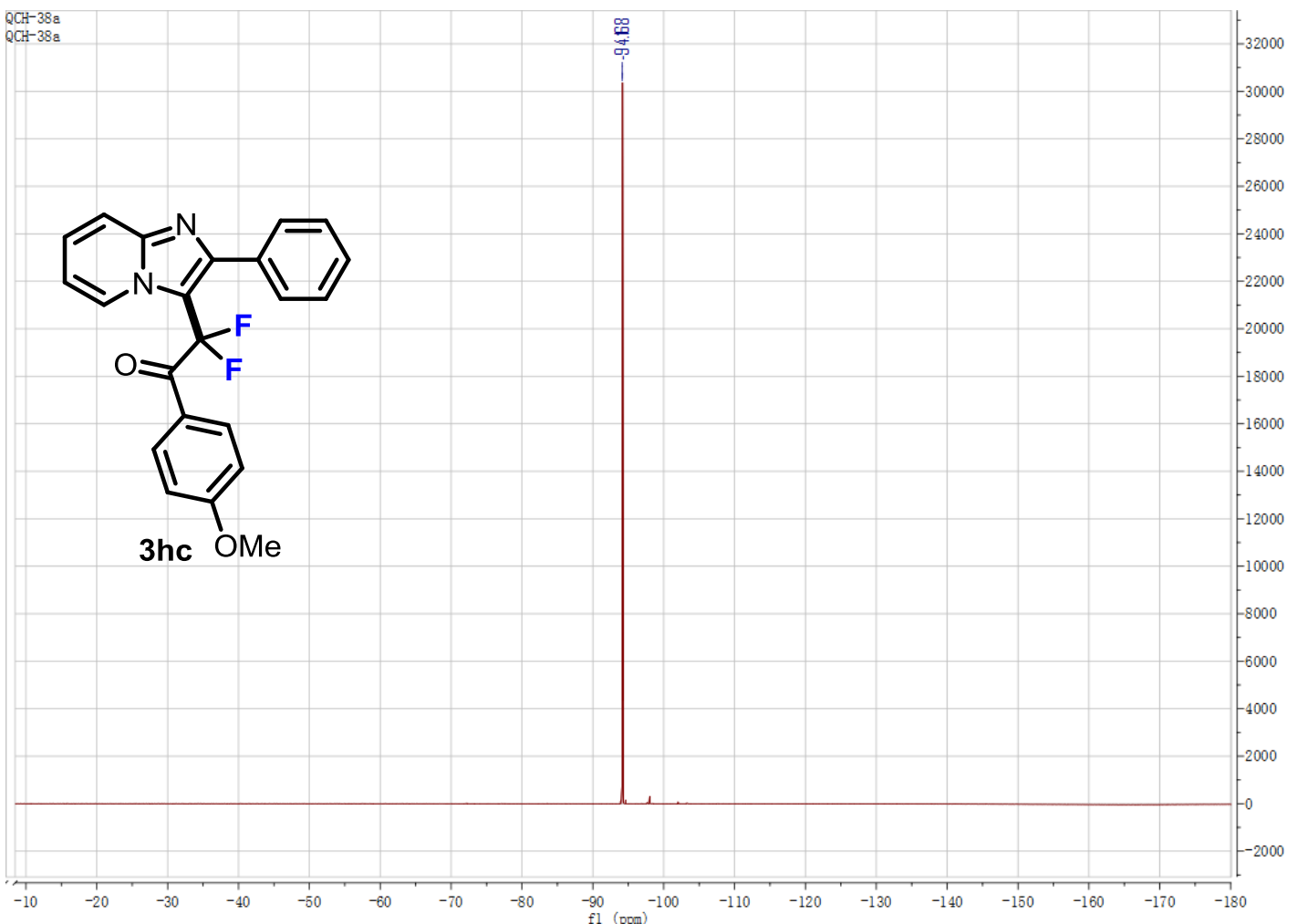

${ }^{13} \mathrm{C}$ NMR (100 MHz, CDCl3): 


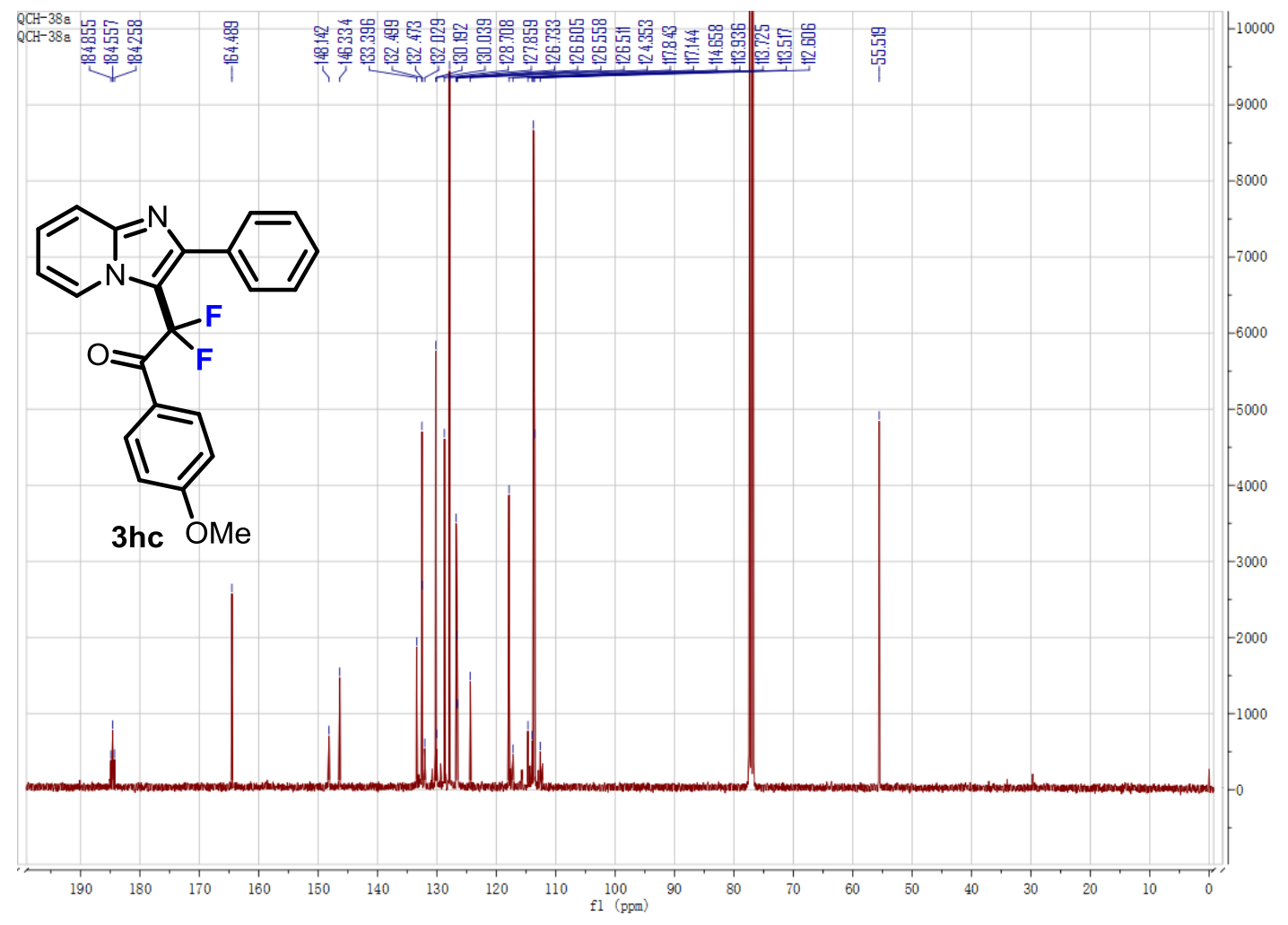


1-(4-(tert-butyl)phenyl)-2,2-difluoro-2-(2-phenylimidazo[1,2-a]pyridin-3-yl)ethan-1-one (3hd)

${ }^{1}$ H NMR (400 MHz, CDCl3):

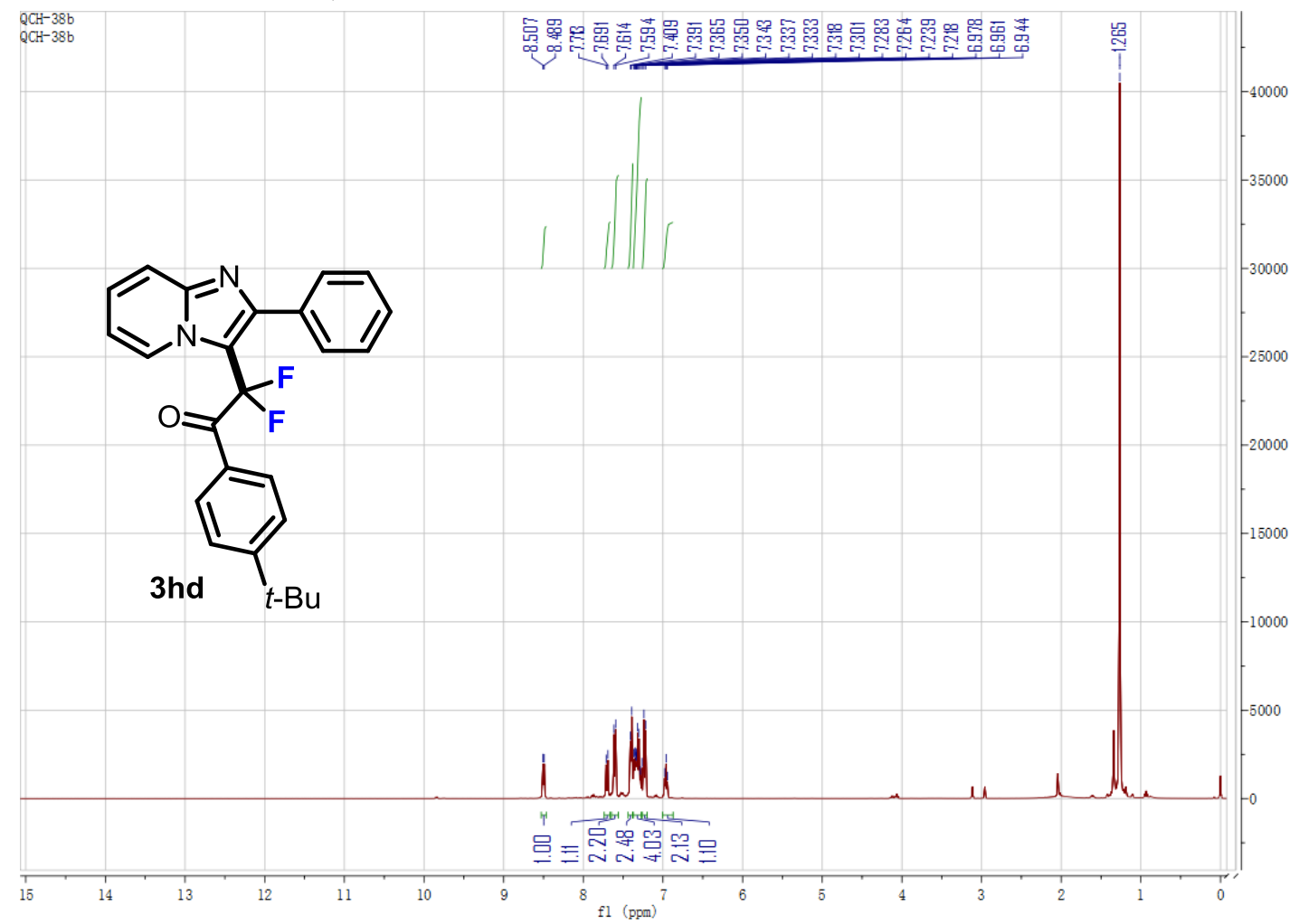

${ }^{19}$ F NMR (376 MHz, CDCl3):

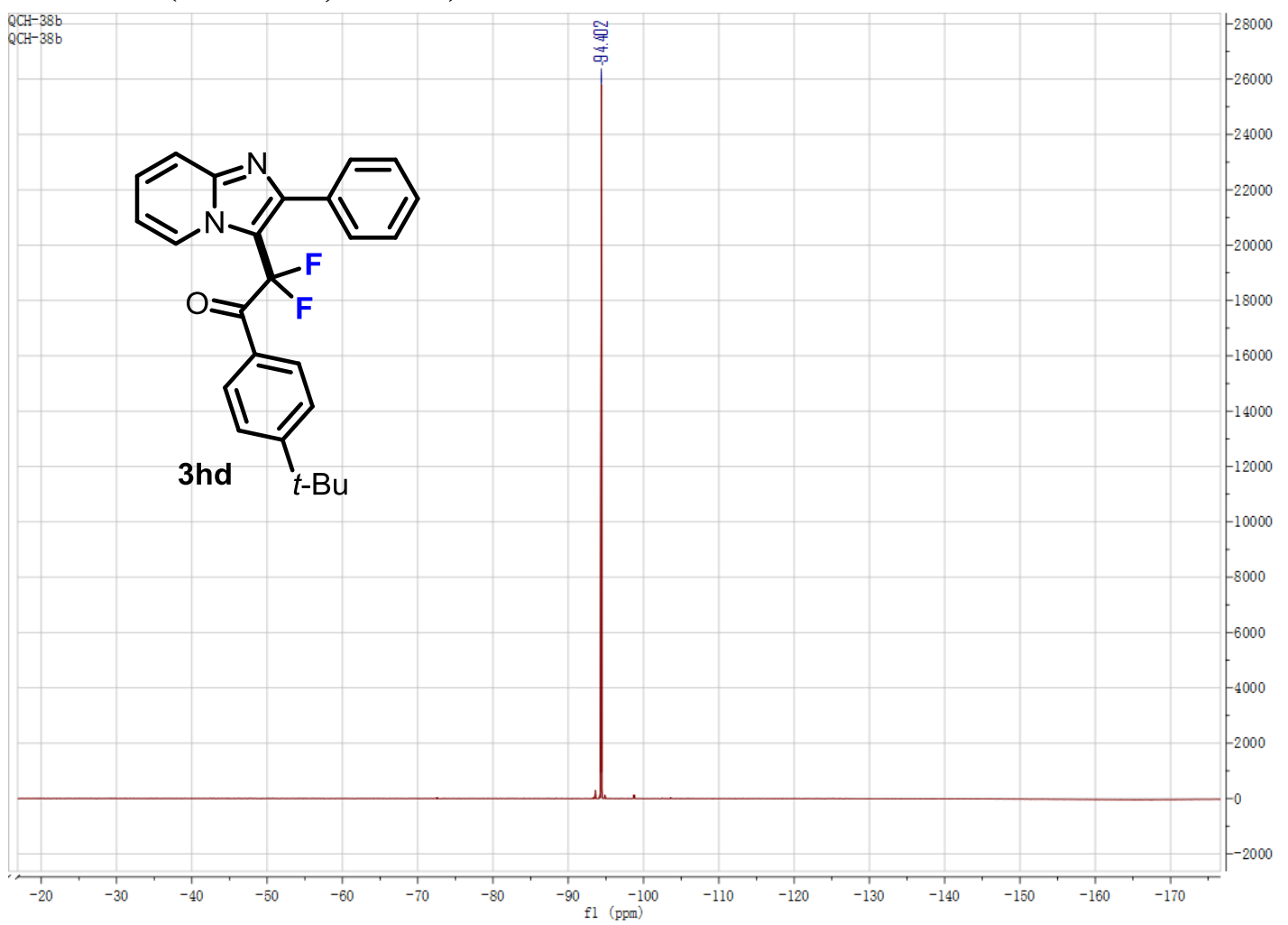


${ }^{13} \mathrm{C}$ NMR (100 MHz, CDCl3):

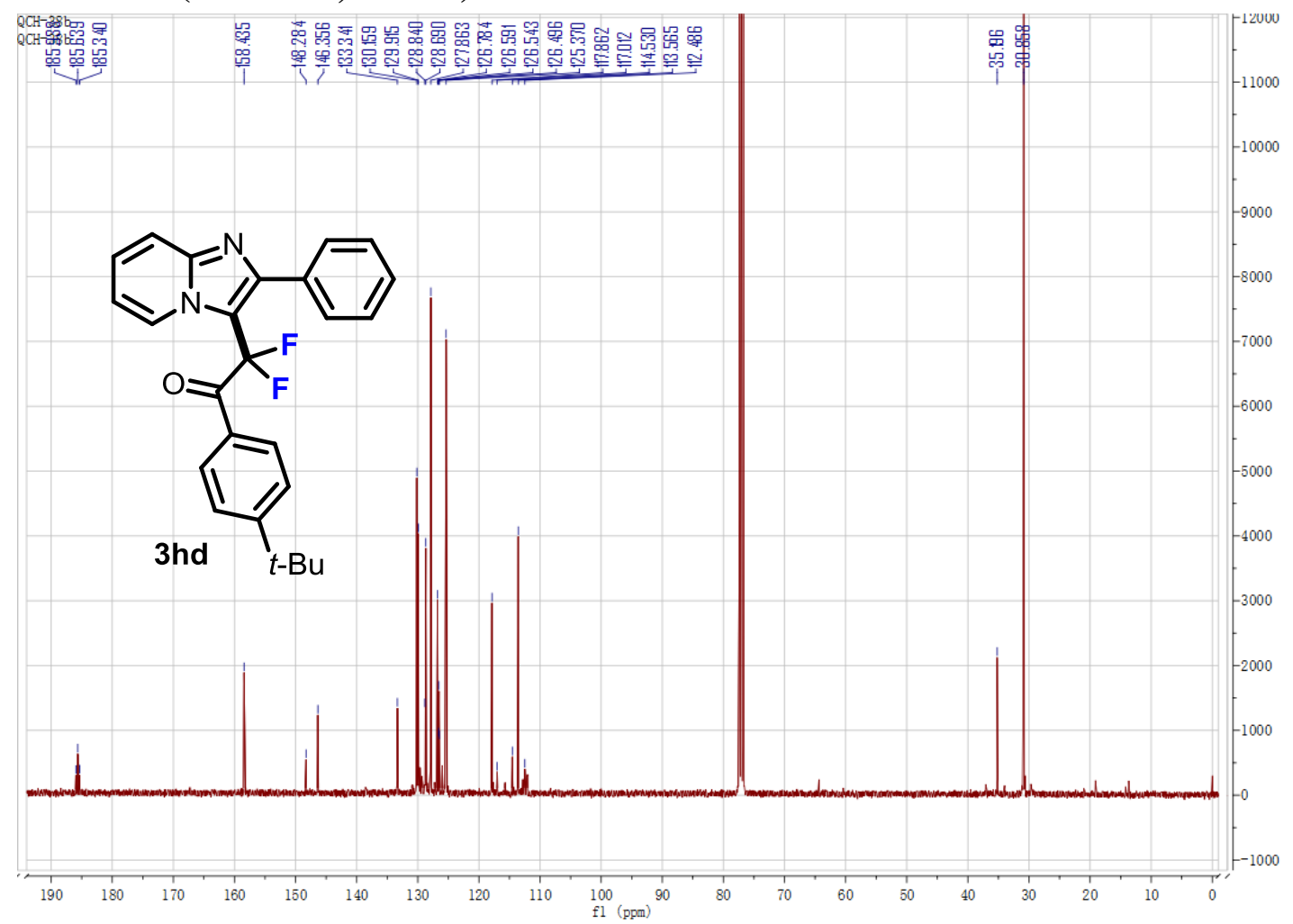


2,2-difluoro-1-(4-fluorophenyl)-2-(2-phenylimidazo[1,2-a]pyridin-3-yl)ethan-1one (3he)

${ }^{1} \mathrm{H}_{\mathrm{NMR}}\left(400 \mathrm{MHz}, \mathrm{CDCl}_{3}\right)$ :

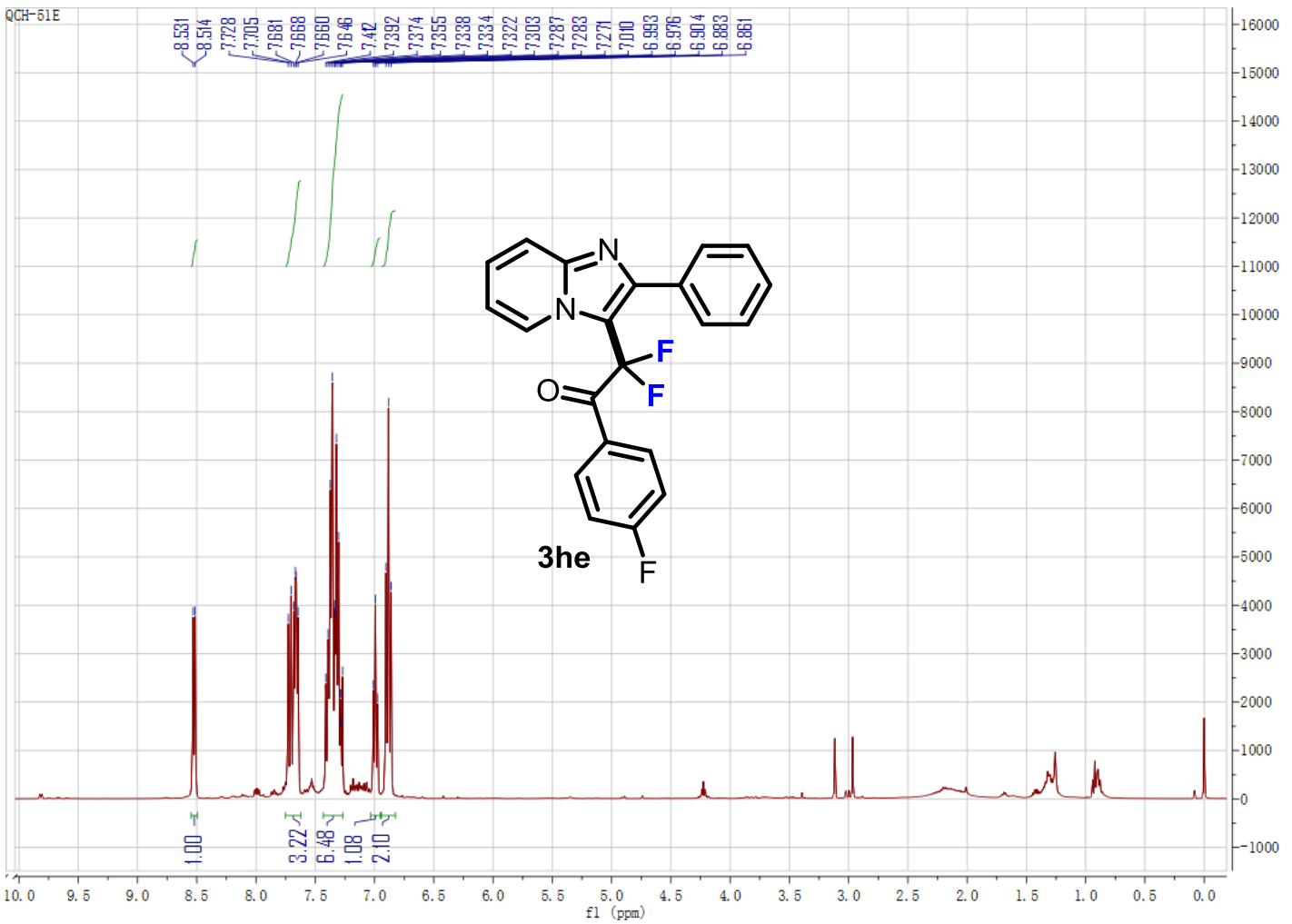

${ }^{19}$ F NMR (376 MHz, CDCl3): 


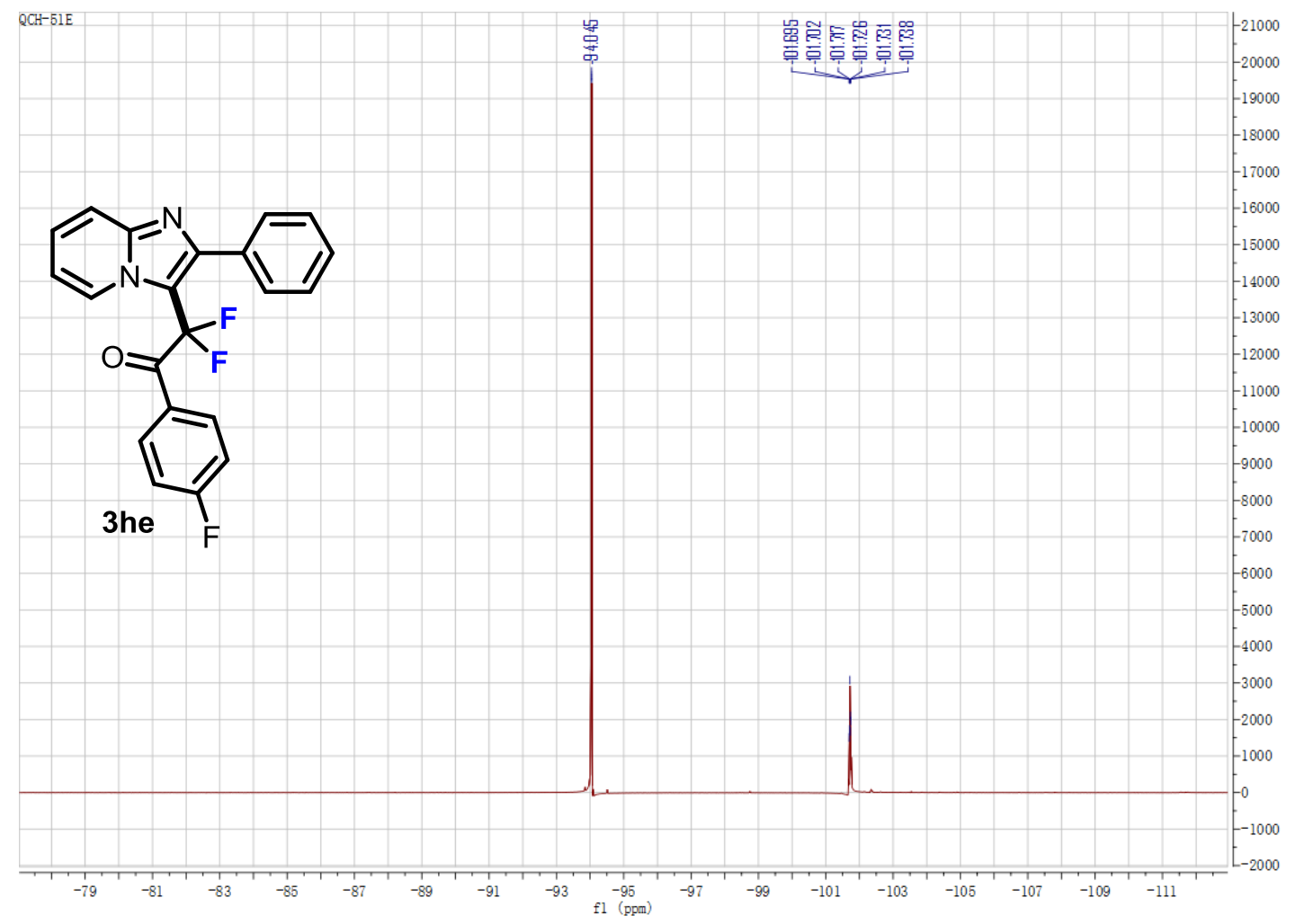

${ }^{13} \mathrm{C}$ NMR (100 MHz, $\left.\mathrm{CDCl}_{3}\right)$ :

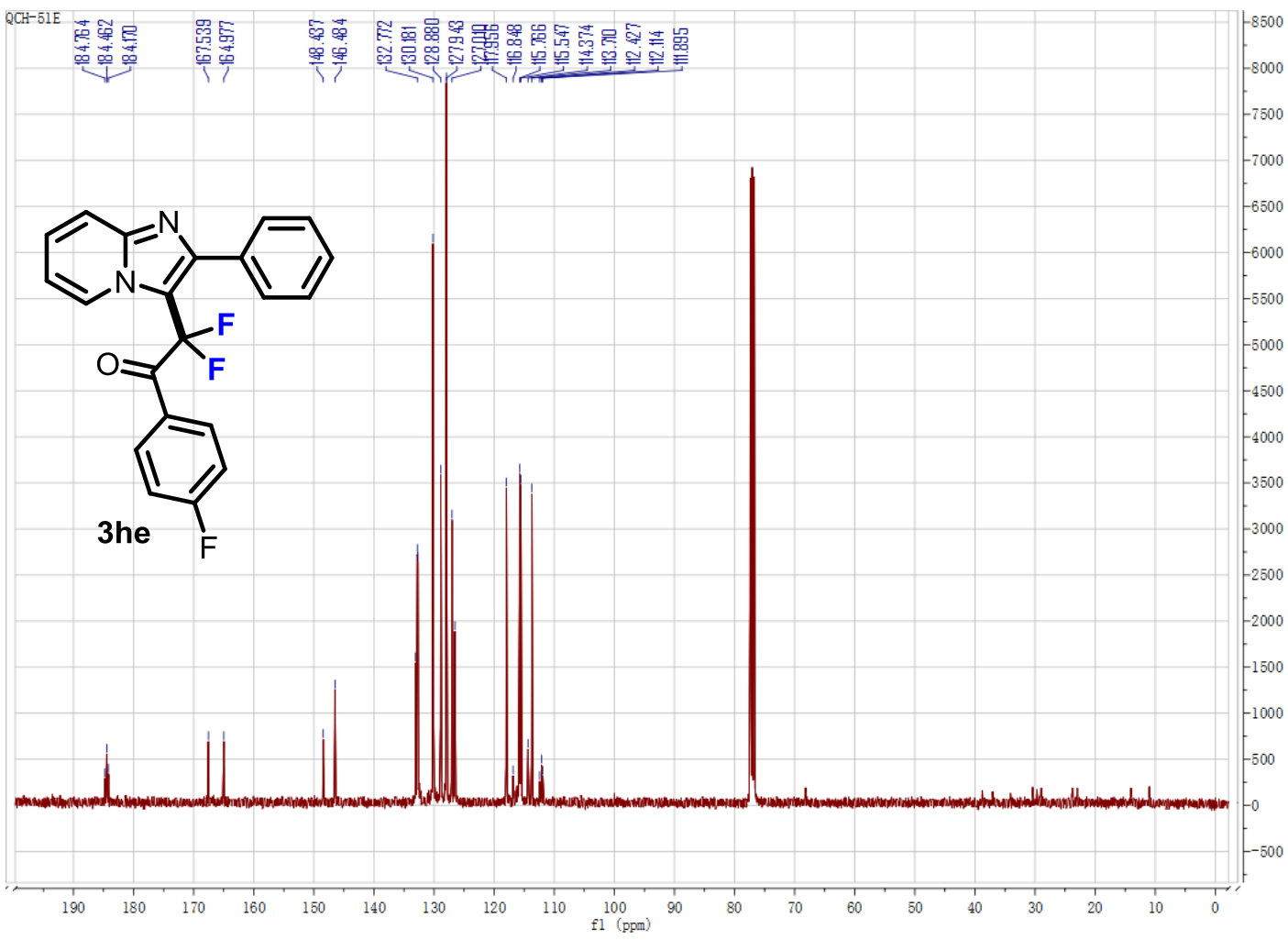


2,2-difluoro-1-(naphthalen-2-yl)-2-(2-phenylimidazo[1,2-a]pyridin-3-yl)ethan-1one (3hf)

${ }^{1} \mathrm{H}$ NMR (400 MHz, CDCl3):

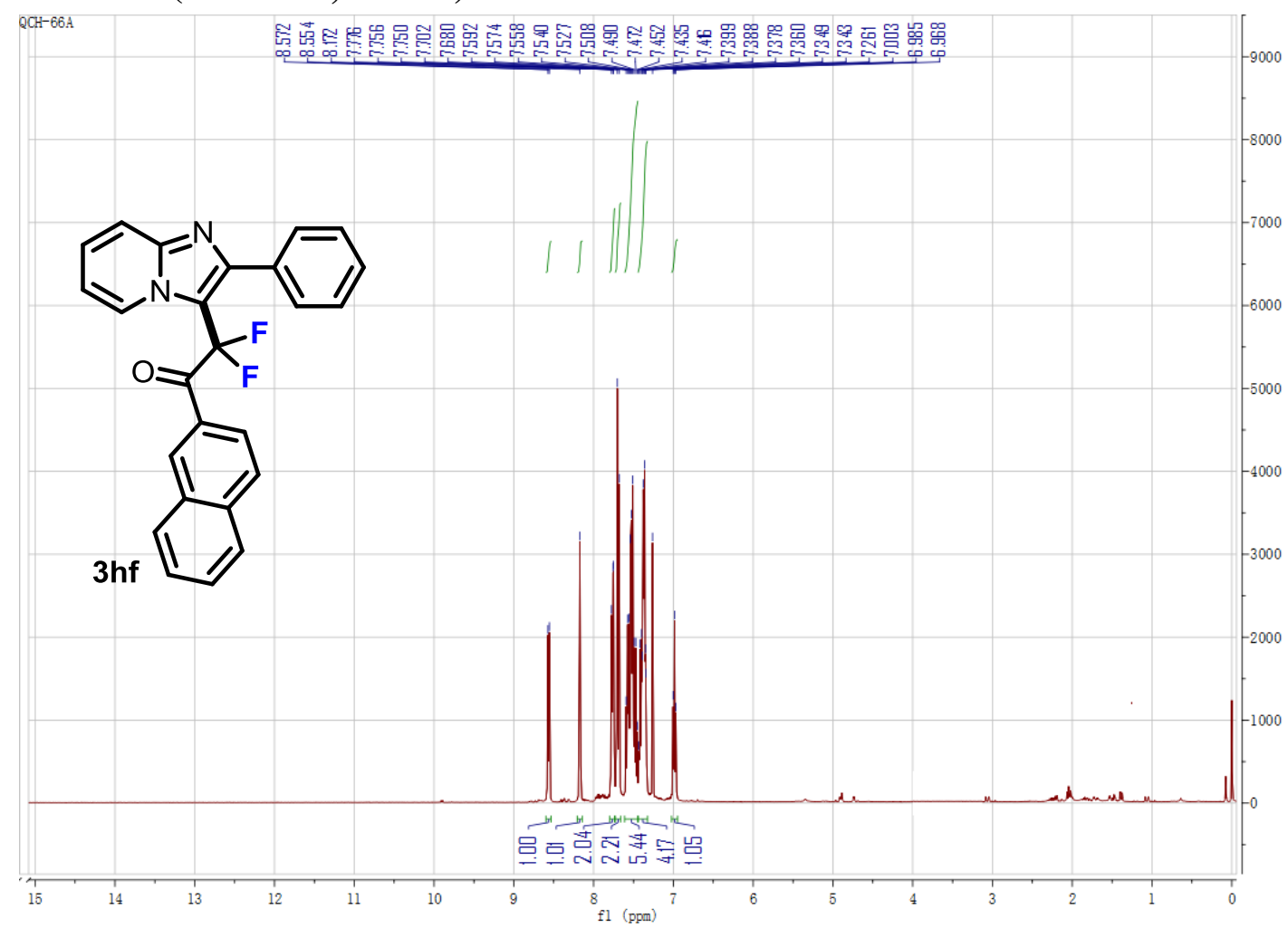

${ }^{19}$ F NMR (376 MHz, CDCl 3$)$ : 


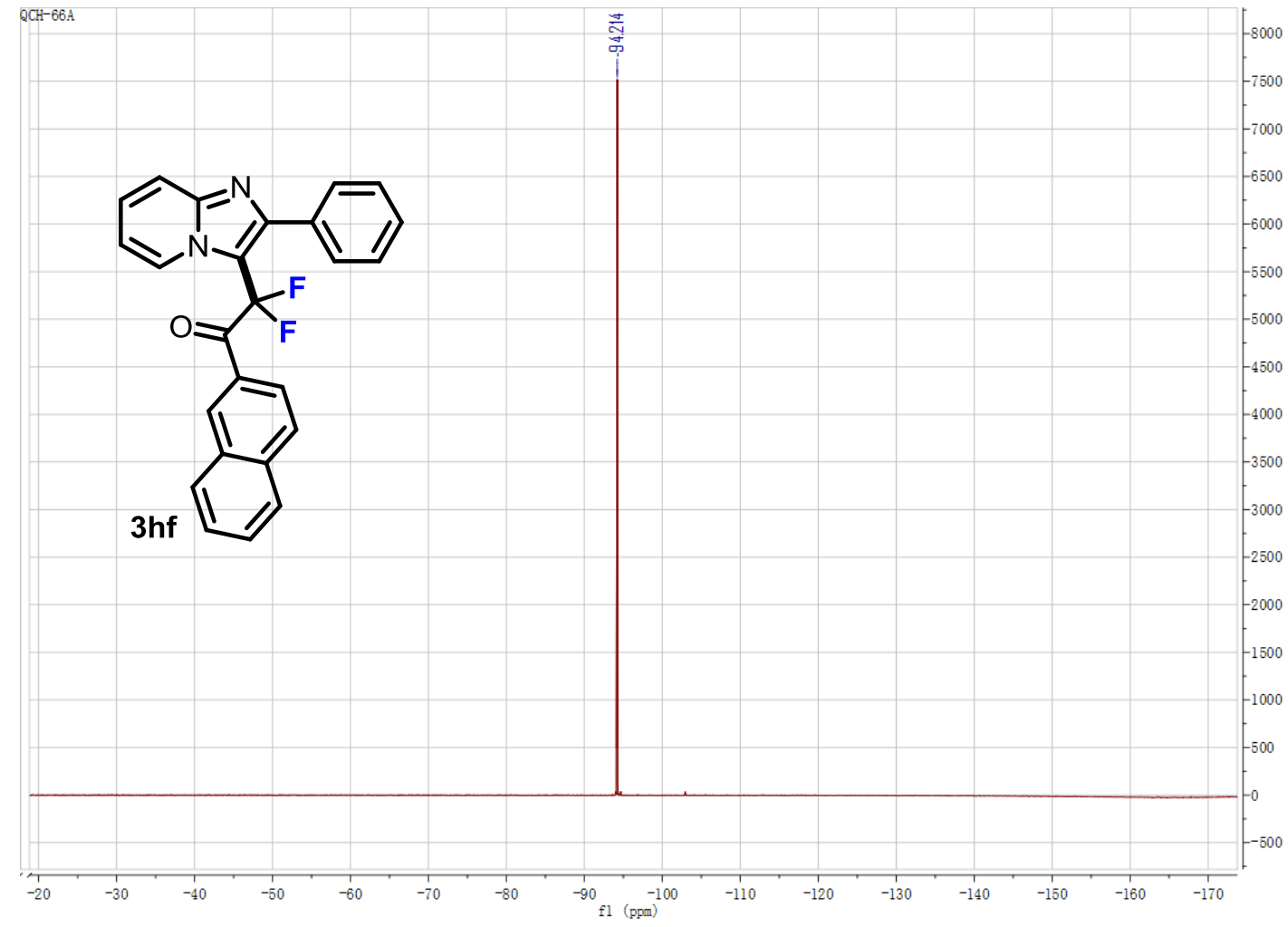

${ }^{13} \mathrm{C}$ NMR (100 MHz, $\left.\mathrm{CDCl}_{3}\right)$ :

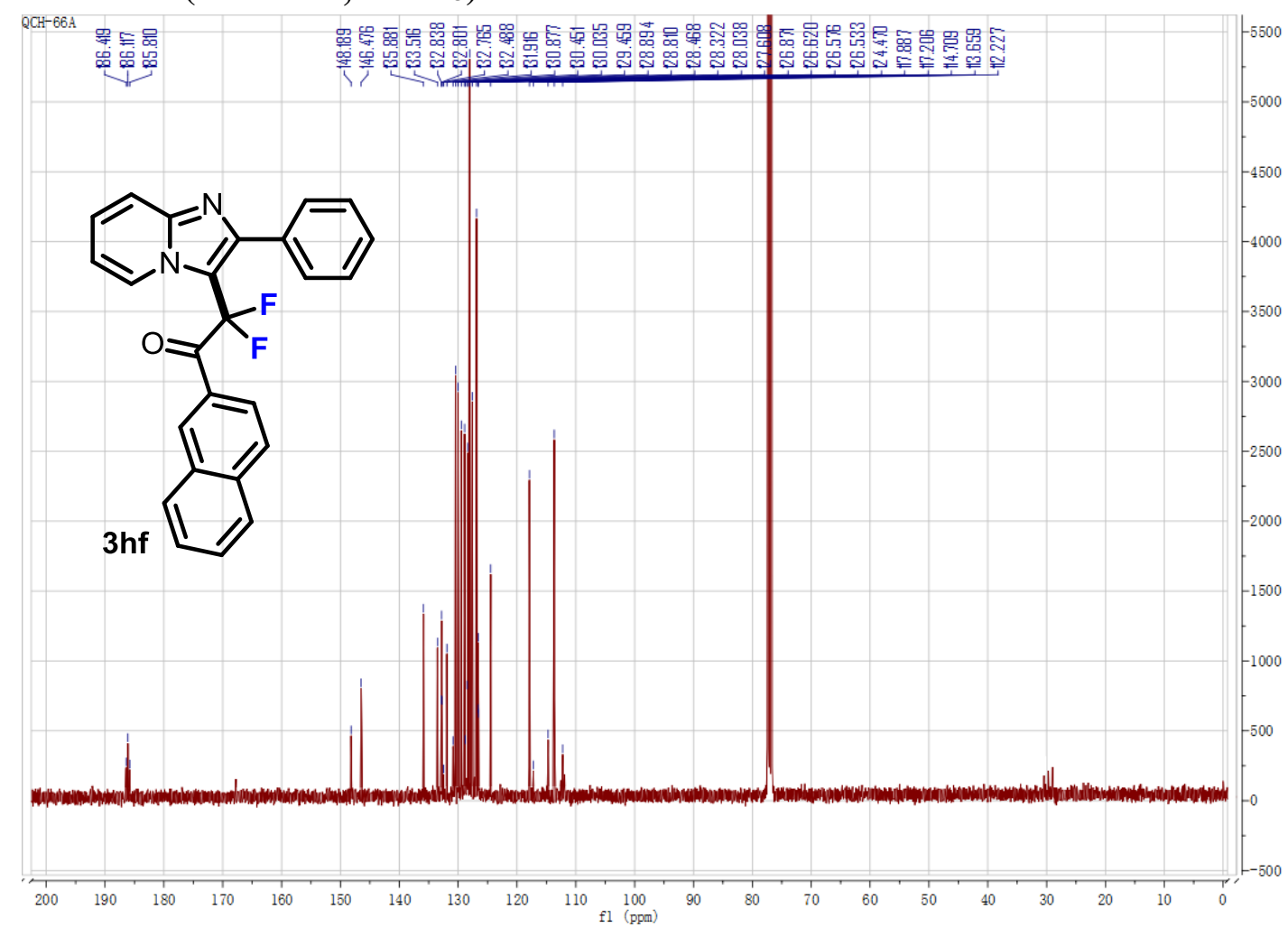


2,2-difluoro-2-(2-phenylimidazo[1,2-a]pyridin-3-yl)-1-(thiophen-2-yl)ethan-1-one (3hg) ${ }^{1} \mathrm{H}$ NMR (400 MHz, CDCl3):

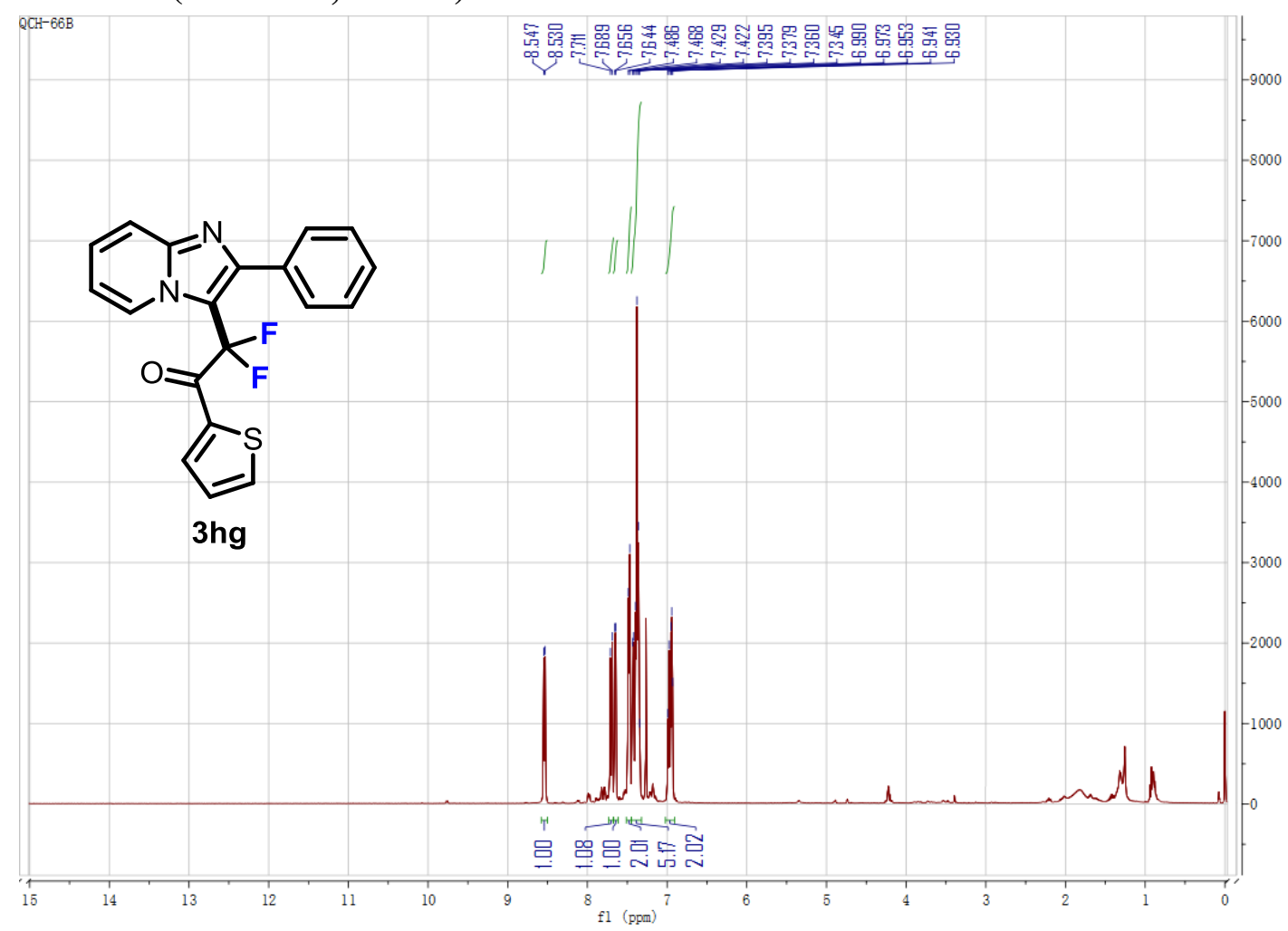

${ }^{19}$ F NMR (376 MHz, CDCl 3$)$ : 


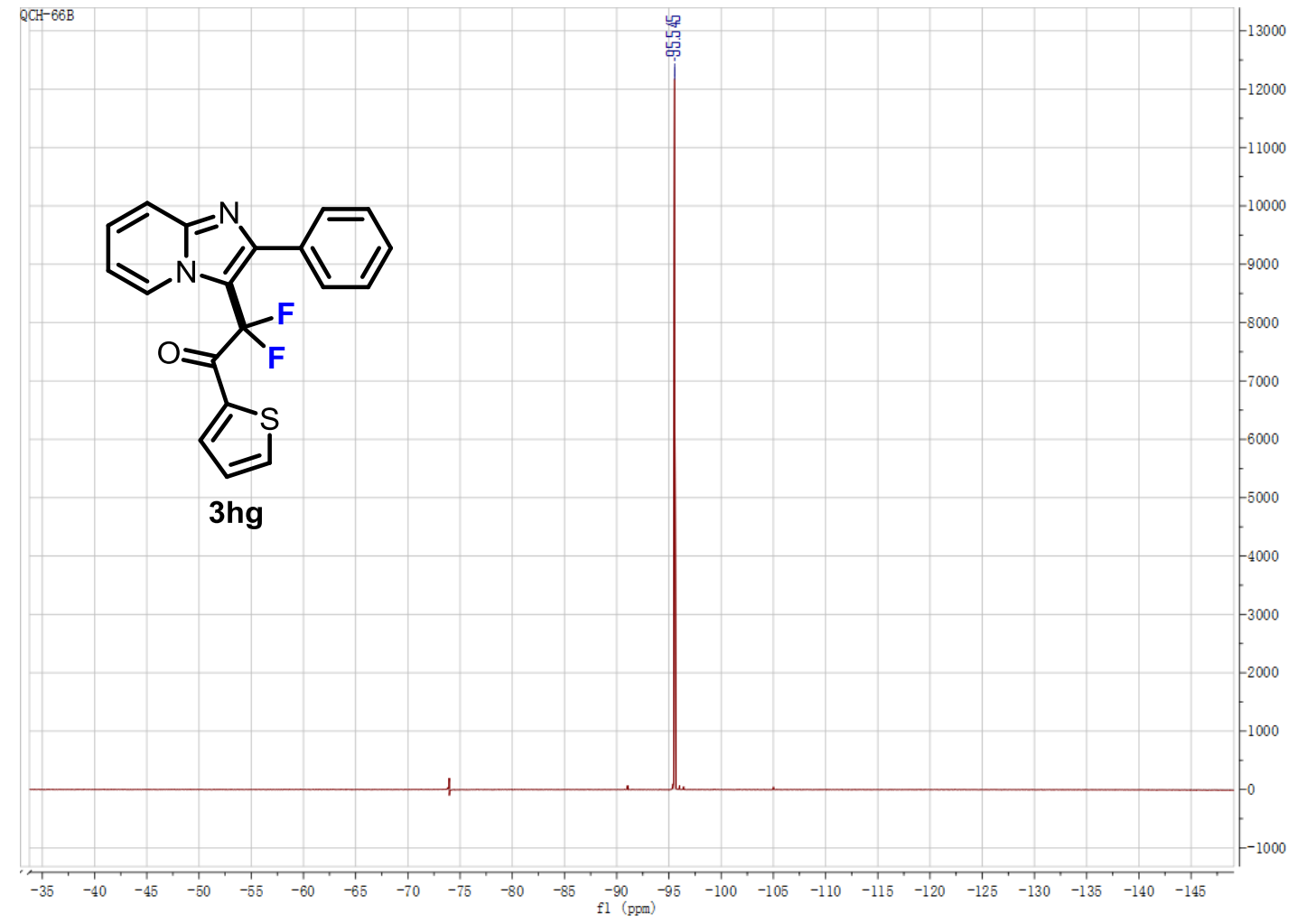

${ }^{13} \mathrm{C}$ NMR (100 MHz, $\left.\mathrm{CDCl}_{3}\right)$ :

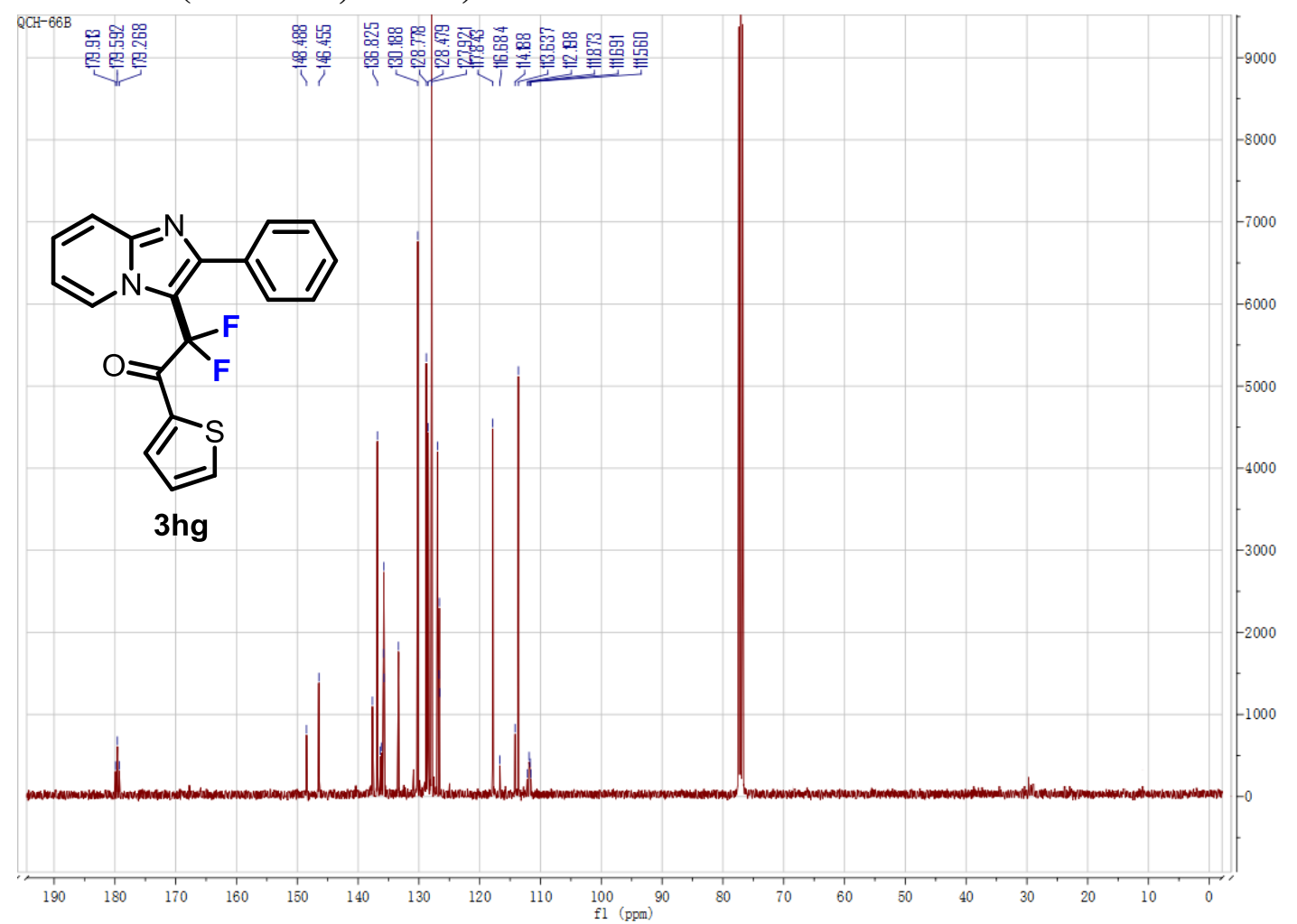


1-(benzo $[d][1,3]$ dioxol-5-yl)-2,2-difluoro-2-(2-phenylimidazo[1,2-a]pyridin-3-yl)ethan-1one (3hh)

${ }^{1}$ H NMR (400 MHz, CDCl3):

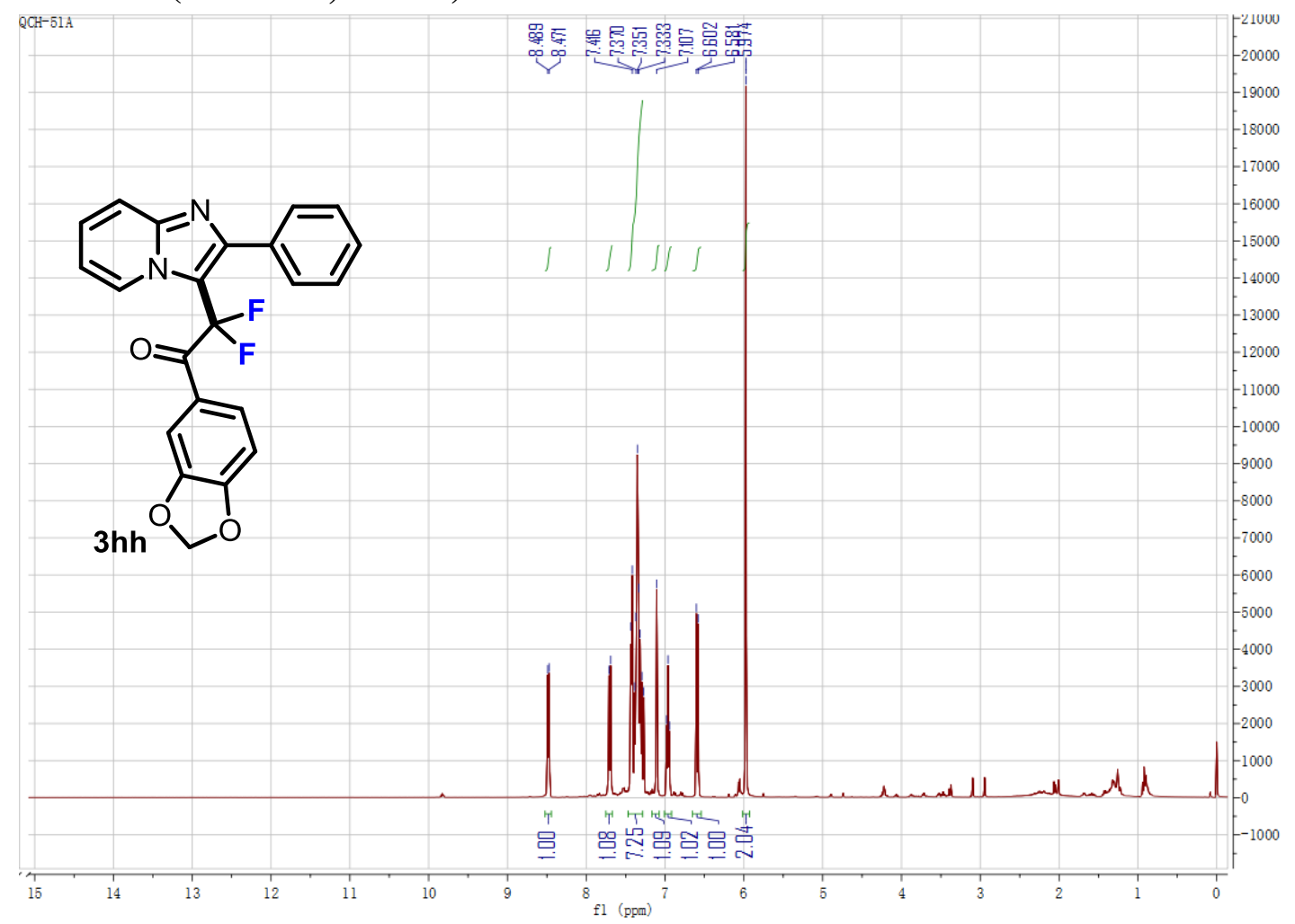

${ }^{19}$ F NMR (376 MHz, CDCl 3$)$ : 


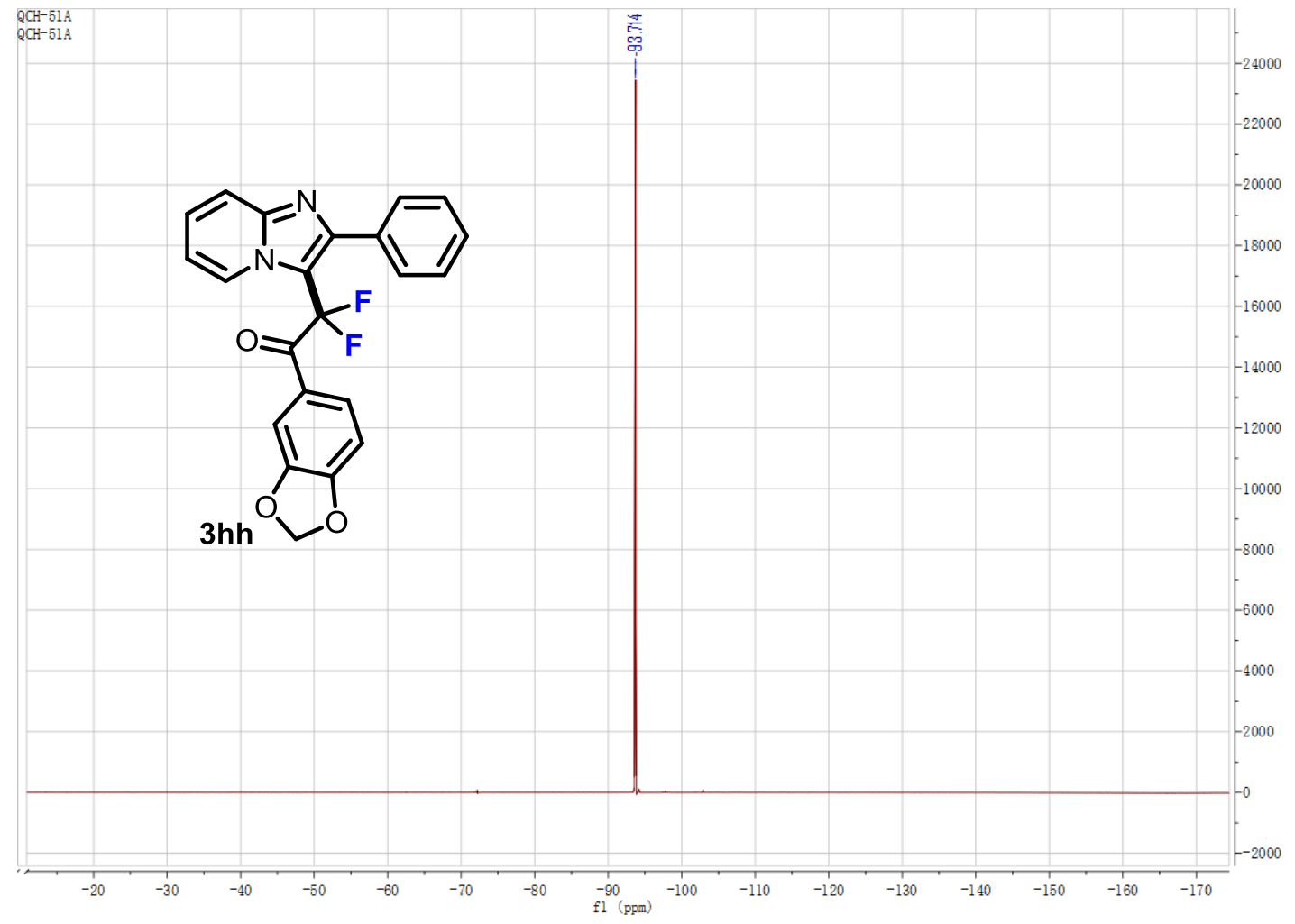

${ }^{13} \mathrm{C}$ NMR (100 MHz, $\left.\mathrm{CDCl}_{3}\right)$ :

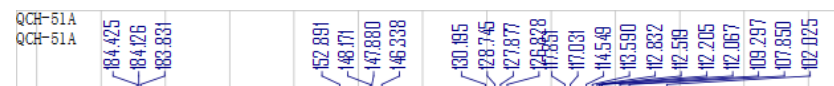

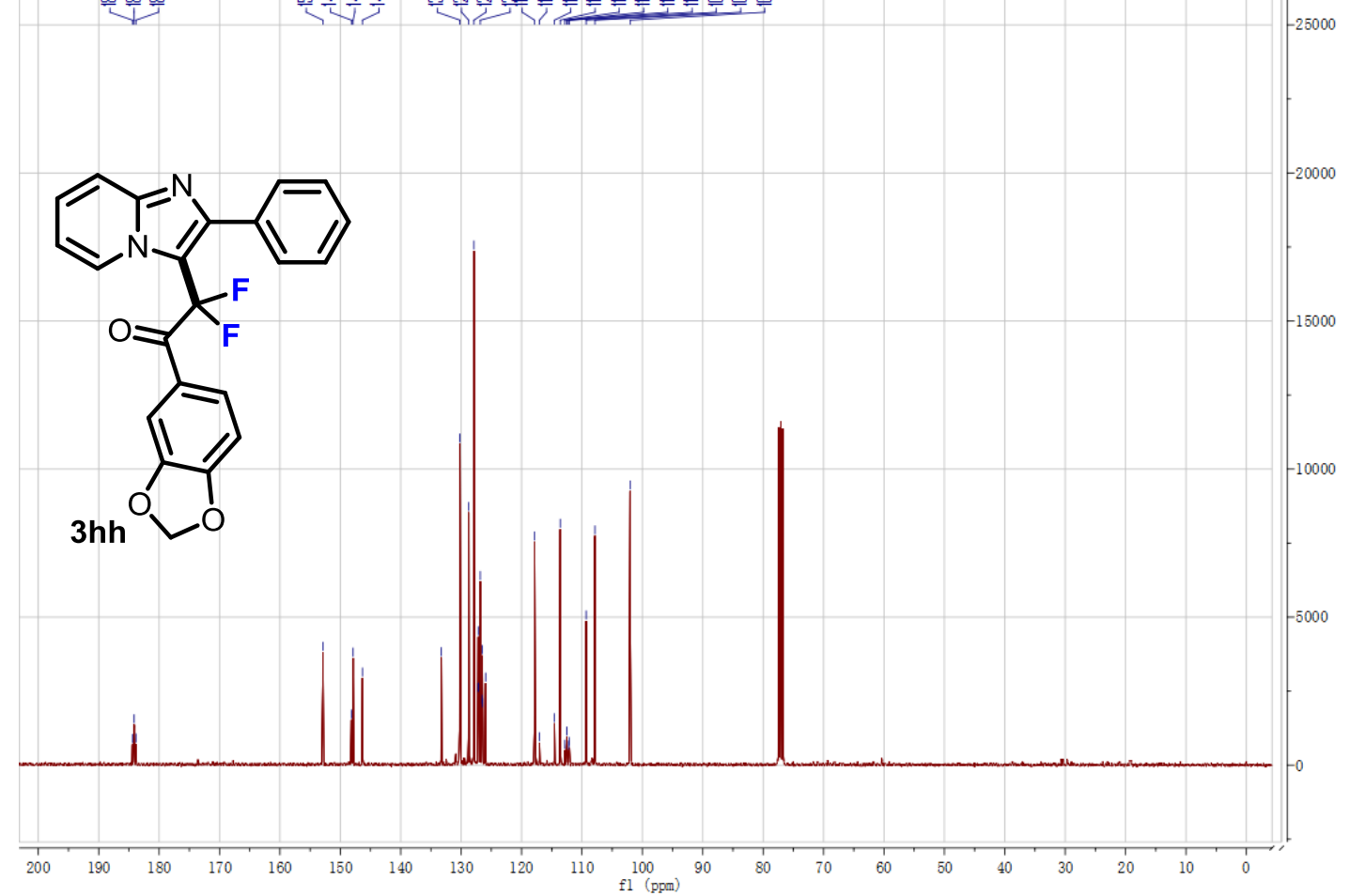


1-(4-(tert-butyl)phenyl)-2,2-difluoro-2-(6-phenylimidazo[2,1-b]thiazol-5-yl)ethan-1-one (3ia)

${ }^{1} \mathrm{H}$ NMR (400 MHz, CDCl3):

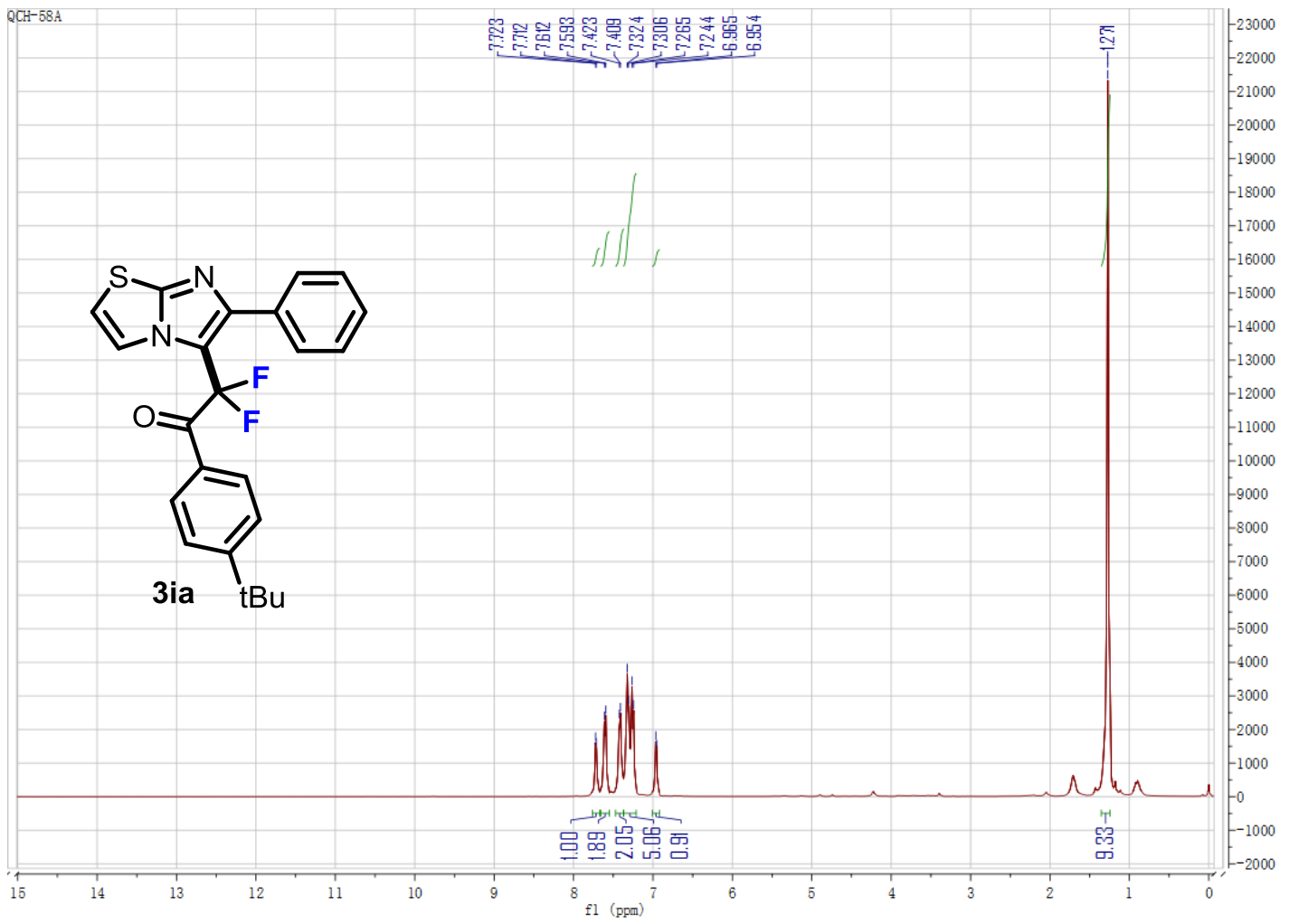


${ }^{19}$ F NMR (376 MHz, CDCl3):

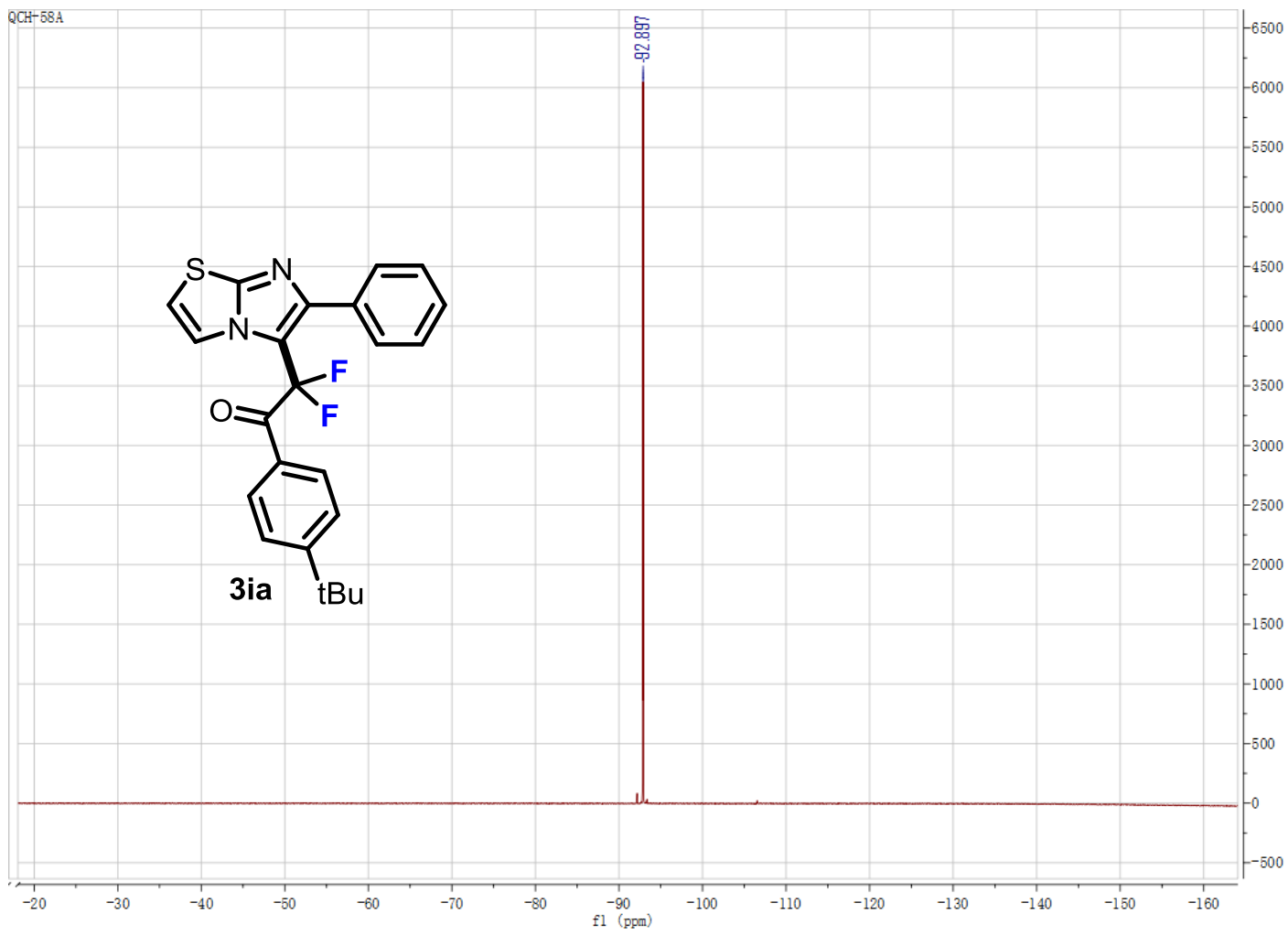

${ }^{13} \mathrm{C}$ NMR (100 MHz, CDCl 3$)$ :

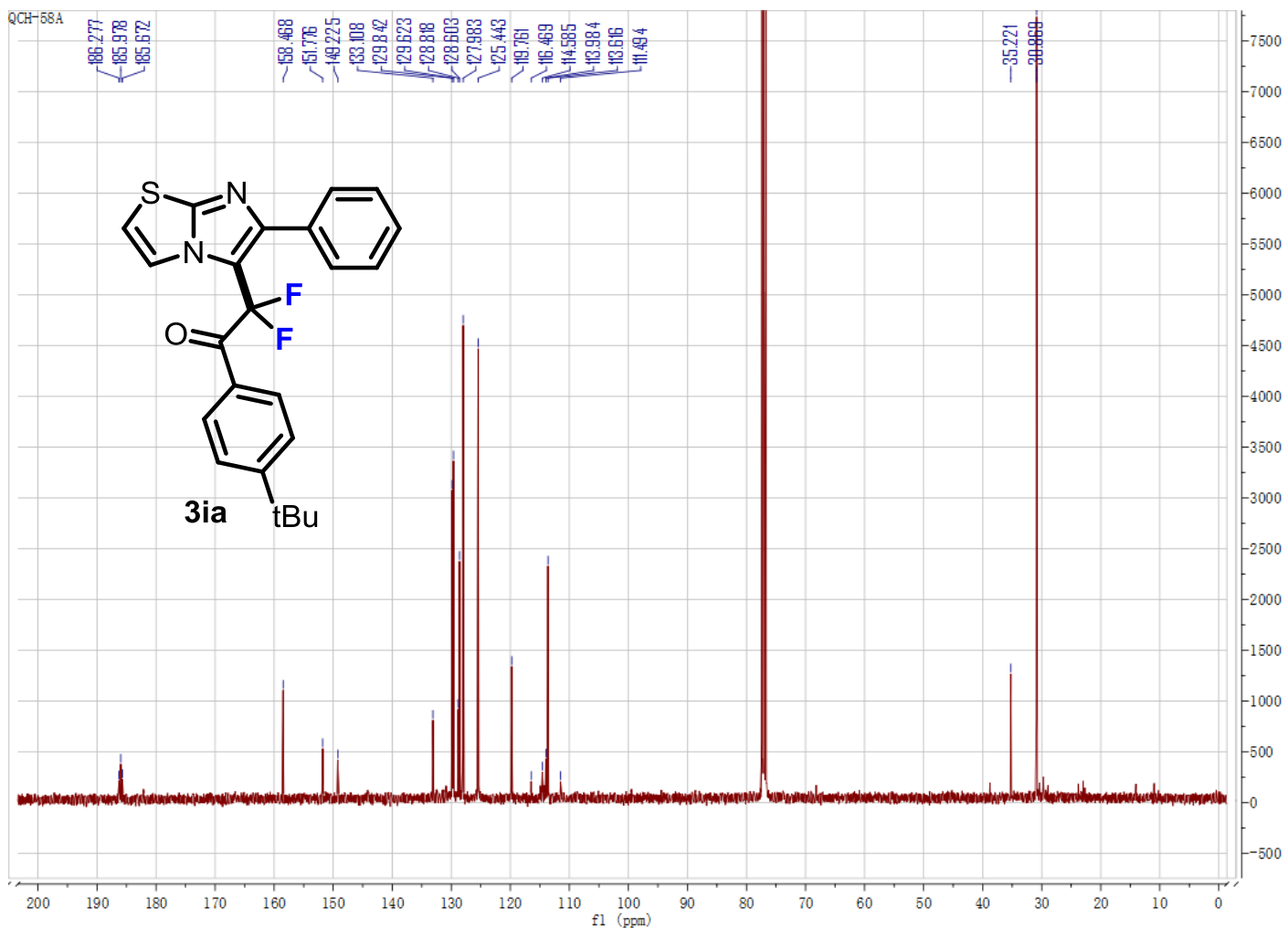


2,2-difluoro-1-(4-methoxyphenyl)-2-(6-phenylimidazo[2,1-b]thiazol-5-yl)ethan-1-one (3ib)

${ }^{1} \mathrm{H}$ NMR (400 MHz, CDCl3):

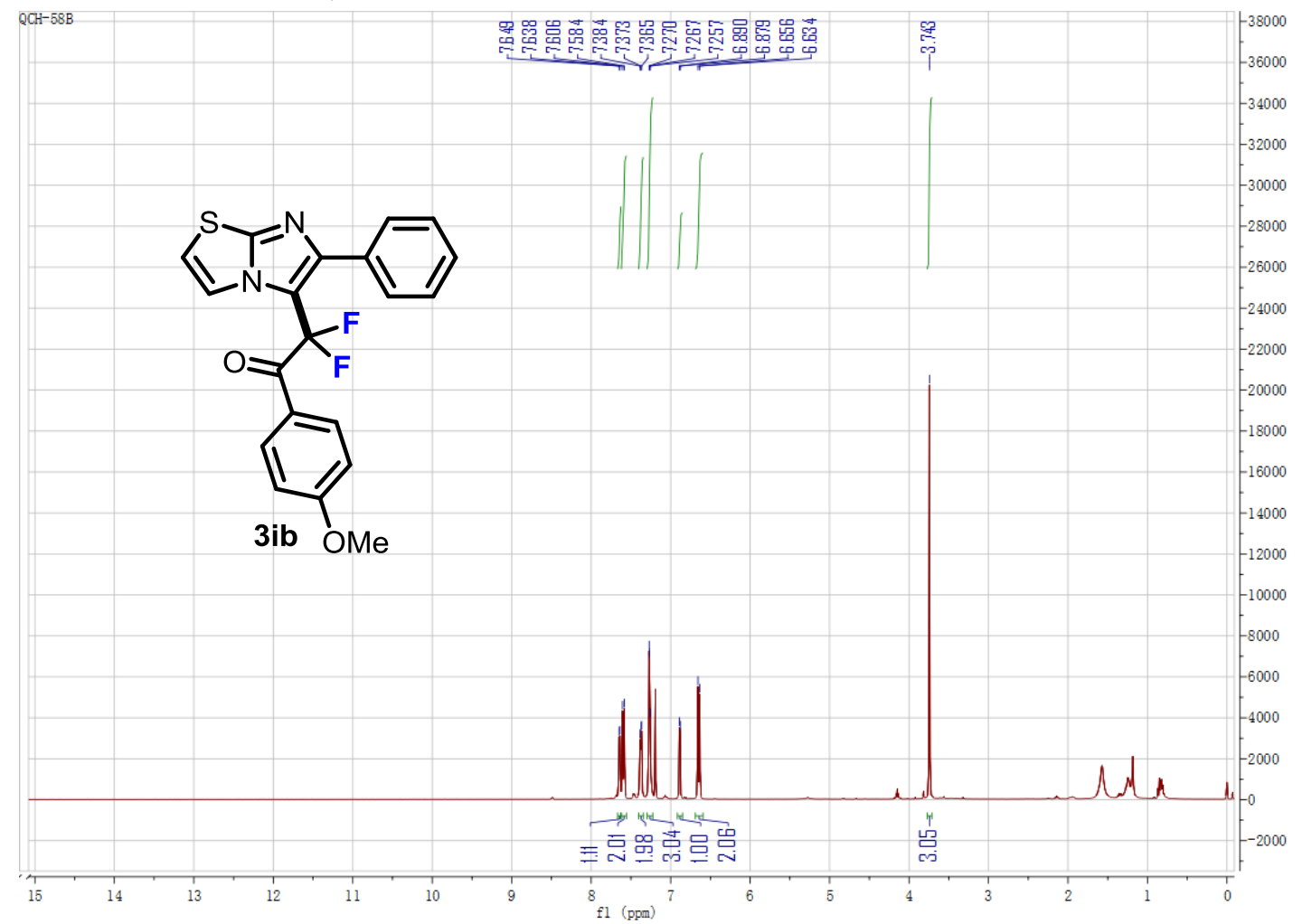


${ }^{19}$ F NMR (376 MHz, CDCl3):

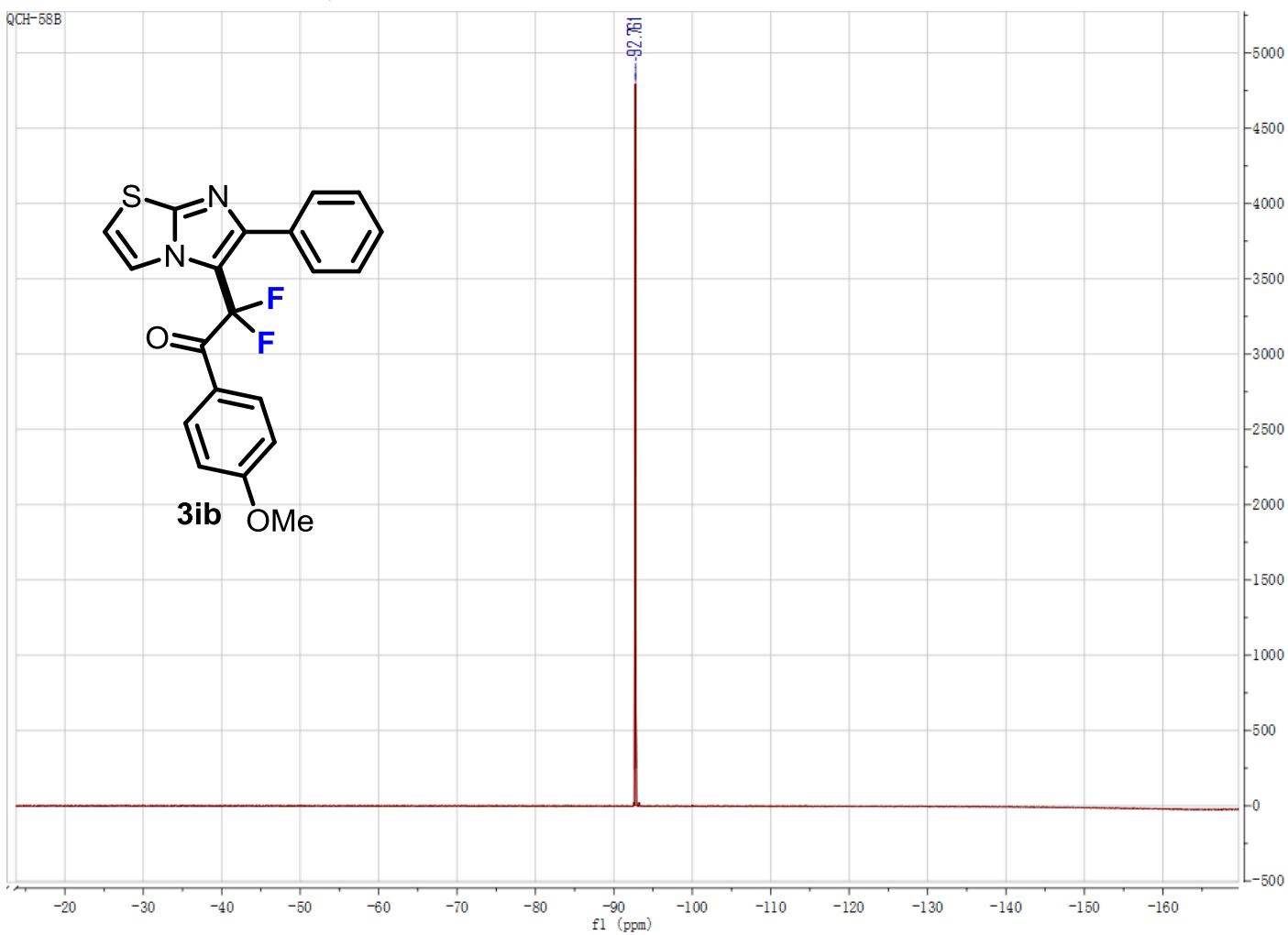

${ }^{13} \mathrm{C}$ NMR (100 MHz, CDCl3):

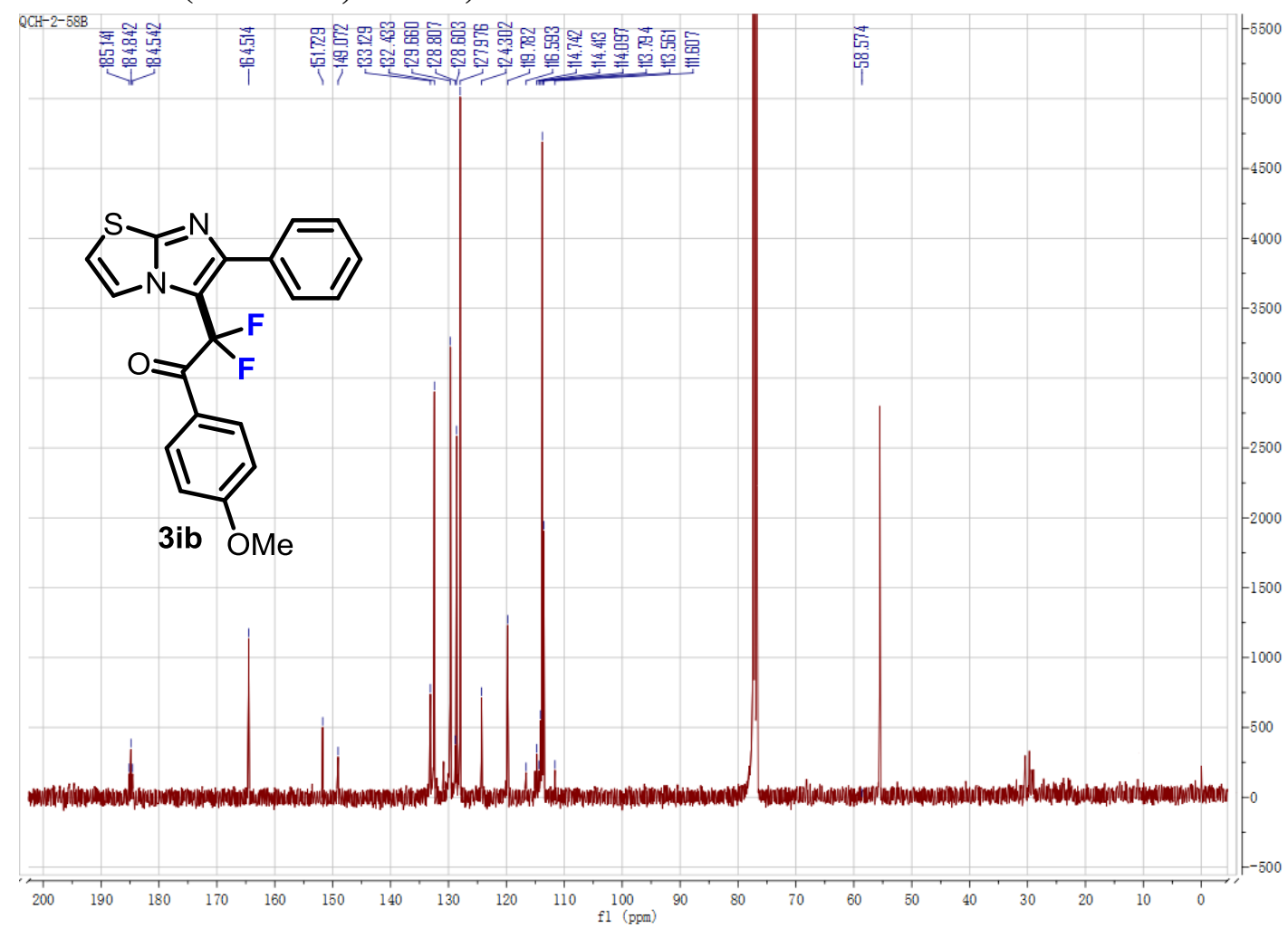


